\title{
Tracking the Wanders of Nature
}

\author{
Clas Veibäck
}


Cover illustration: Dolphins, birds and an iceberg (and maybe a rhino)

Linköping studies in science and technology. Dissertations

No. 1958

\section{Tracking the Wanders of Nature}

Clas Veibäck

clas.veibackeliu.se

www.control.isy.liu.se

Division of Automatic Control

Department of Electrical Engineering

Linköping University

SE-581 83 Linköping

Sweden

ISBN 978-91-7685-200-2

ISSN 0345-7524

Copyright (c) 2018 Clas Veibäck

Printed by LiU-Tryck, Linköping, Sweden 2018 
To Mia, Sam and Stina 



\section{Abstract}

Target tracking is a mature topic with over half a century of mainly military and aviation research. The field has lately expanded into a range of civilian applications due to the development of cheap sensors and improved computational power. With the rise of new applications, new challenges emerge, and with better hardware there is an opportunity to employ more elaborated algorithms.

There are five main contributions to the field of target tracking in this thesis. Contributions I-IV concern the development of non-conventional models for target tracking and the resulting estimation methods. Contribution V concerns a reformulation for improved performance. To show the functionality and applicability of the contributions, all proposed methods are applied to and verified on experimental data related to tracking of animals or other objects in nature.

In Contribution I, sparse Gaussian processes are proposed to model behaviours of targets that are caused by influences from the environment, such as wind or obstacles. The influences are learned online as a part of the state estimation using an extended Kalman filter. The method is also adapted to handle time-varying influences and to identify dynamic systems. It is shown to improve accuracy over the nearly constant velocity and acceleration models in simulation. The method is also evaluated in a sea ice tracking application using data from a radar on Svalbard.

In Contribution II, a state-space model is derived that incorporates observations with uncertain timestamps. An example of such observations could be traces left by a target. Estimation accuracy is shown to be better than the alternative of disregarding the observation. The position of an orienteering sprinter is improved using the control points as additional observations.

In Contribution III, targets that are confined to a certain space, such as animals in captivity, are modelled to avoid collision with the boundaries by turning. The proposed model forces the predictions to remain inside the confined space compared to conventional models that may suffer from infeasible predictions. In particular the model improves robustness against occlusions. The model is successfully used to track dolphins in a dolphinarium as they swim in a basin with occluded sections.

In Contribution IV, an extension to the jump Markov model is proposed that incorporates observations of the mode that are state-independent. Normally, the mode is estimated by comparing actual and predicted observations of the state. However, sensor signals may provide additional information directly dependent on the mode. Such information from a video recorded by biologists is used to estimate take-off times and directions of birds captured in circular cages. The method is shown to compare well with a more time-consuming manual method.

In Contribution V, a reformulation of the labelled multi-Bernoulli filter is used to exploit a structure of the algorithm to attain a more efficient implementation. Modern target tracking algorithms are often very demanding, so sound approximations and clever implementations are needed to obtain reasonable computational performance. The filter is integrated in a full framework for tracking sea ice, from pre-processing to presentation of results. 



\section{Populärvetenskaplig sammanfattning}

Målföljning (eng. target tracking) är ett välutforskat ämne med en historia som sträcker sig tillbaka till åtminstone 30-talet. Då tävlade en handfull nationer om att snabbast kunna upptäcka fienden innan det var för sent. Traditionellt sett har målföljning fortsatt att vara starkt förknippat med militära tillämpningar och flygfart. Det är först på senare år som billiga och kommersiellt tillgängliga sensorer har öppnat upp för en mängd betydligt fredligare användningsområden.

Målföljning skulle kunna beskrivas som lokalisering av främmande objekt genom att samla in data från sensorer. Den här avhandlingen behandlar framförallt målföljning av olika sorters djur där data samlas in med videokameror. Det finns två bakomliggande syften. Det ena handlar om att underlätta forskning för biologer och det andra handlar om att skapa tekniska lösningar för att underlätta skyddet av sällsynta djur. Även målföljning av drivis där data samlas in med radar behandlas. Trots den vitt skilda tillämpningen är många metoder desamma. Syftet är att hantera drivis i norra ishavet där detektion och målföljning är viktiga komponenter för att undvika kollisioner.

Biologer lägger ofta en ansenlig mängd tid på att samla in, annotera och sortera data. Det är tid som kan spenderas på mer givande forskningsaktiviteter. Med videokamera, bildbehandling och moderna algoritmer för målföljning är det möjligt att i viss mån automatisera datainsamlingen. Med automatisering kan mer information samlas in än med traditionella metoder och längre experiment kan ofta genomföras. Ytterligare en fördel är att man kan minska påverkan på djuren.

Parkvakterna i många nationalparker kämpar dagligen med intrång från tjuvjägare. De har ytterst begränsade resurser och utsätter sina liv för stor fara. Bestånden minskar fortfarande för många djurarter som går en mörk framtid till mötes. För att vända trenden behövs stora insatser på många fronter samtidigt. Målföljning kan bidra med att på ett kostnadseffektivt sätt tillhandahålla övervakning av nationalparker. Kännedom om var djuren befinner sig underlättar koordinering av parkvakternas insatser för att skydda djuren. Målföljning kan ske med ett flertal olika sensorer, såsom radarer, fast uppsatta och luftburna videokameror, mikrofoner som lyssnar efter djurläten och även vittnesmål från parkvakterna. All insamlad information bidrar till att skapa en helhetsbild av situationen i nationalparken om den används rätt.

Ishantering är ett viktigt område för oljeindustrin för att garantera säkerhet och undvika allvarliga olyckor. Målet är att upptäcka och spåra is som flyter i havet och om nödvändigt vidta åtgärder för att undvika kollision. Målet är att i förlängningen sätta upp ett stort nätverk av olika sensorer och databaser för att få en heltäckande bild av det aktuella läget. Flera källor diskuteras, såsom markoch fartygsradarer av olika slag, satelliter, drönare med kameror och väderdatabaser.

Att skapa fullständiga och användbara lösningar för biologer, parkvakter och oljeindustrin är väldigt ambitiösa mål. I avhandlingen presenteras bakomliggande teori för målföljning varvat med författarens egna forskningsbidrag och lös- 
ningar för en handfull specifika problem och tillämpningar.

Det första projektet som presenteras är ett samarbete med Kolmårdens djurpark. Biologer i djurparken studerar delfiners beteende i fångenskap. I dagsläget markerar studenter för hand i video var delfinerna befinner sig i bassängen. Med målföljning samlas djurens positioner in automatiskt utan mänsklig inblandning. Det främsta bidraget i forskningen är utvecklingen av en modell för hur delfinerna rör sig i bassängen.

Det andra projektet som presenteras är ett samarbete med biologer vid Lunds universitet som studerar beteendet hos flyttfåglar. I en metod från 60-talet mäts fåglars rörelser i en tratt. Från repor i tratten som orsakats vid fåglarnas lyftförsök analyserar man riktningarna för lyftförsöken. Med videokamera och målföljning samlas djurens positioner in och enskilda lyftförsök detekteras automatiskt. Det främsta bidraget i forskningen är en metod för att bättre utnyttja information från videon till att detektera lyftförsöken.

Det tredje projektet som presenteras är ett samarbete med Smarta Savanner. En idé som utforskas är möjligheten att använda parkvakternas vittnesmål om spår från noshörningar för att förbättra målföljningen. Å ena sidan är data från videokameror och radarer väldigt noggranna i tid, men relativt osäkra i de uppmätta positionerna. Å andra sidan kan positionen för ett spår mätas noggrant samtidigt som det ofta är svårt att avgöra när noshörningen var på platsen. Genom att utnyttja informationen från båda källorna kan noshörningars förflyttningar i parken kartläggas bättre. Den bakomliggande teorin för observationer med osäker tid inom målföljning är relativt outforskad. Det främsta bidraget i forskningen är utvecklingen av en metod för att utnyttja sådana observationer. Enkla simulerade fall används för att analysera metoden. Metoden utvärderas även i en tillämpning för att förbättra den satellitbaserade positionsbestämningen av en orienterare genom att noggrant mäta positionen på kontrollerna.

Det fjärde projektet som presenteras är ett samarbete med Norges teknisk-naturvitenskapelige universitet (NTNU) och Norut i Norge som samlat in radardata på Svalbard. Det främsta bidraget är utvecklandet av en metod som lär sig hur lokala strömmar och vindar påverkar drivisen för att bättre kunna förutspå rörelser. Ett annat bidrag i forskningen är en förenkling av formuleringen och implementationen av en modern algoritm för målföljning.

Projekten, som alla har flera likheter och skillnader med varandra, kan gemensamt sammanfattas med att de spårar rörelser, eller vandringar, $i$ naturen. 


\section{Acknowledgments}

I would like to express my gratitude to my supervisor Dr. Gustaf Hendeby for always having time for discussions, day and night, despite a high workload. I am also thankful to my co-supervisor Prof. Fredrik Gustafsson for your neverending optimism and resourcefulness. I want to thank my room mate Dr. Martin Skoglund for all the rewarding and fun discussions on any and all topics over the years.

I thank Dr. Martin Enqvist, Prof. Svante Gunnarsson and Ninna Stensgård for maintaining an amazing work environment. Working in the Automatic Control group is an absolute pleasure.

All the comments, feedback and proofreading provided by Dr. Gustaf Hendeby, Per Boström, Jonatan Olofsson, Dr. Martin Skoglund, Dr. Zoran Sjanic, Dr. Rickard Karlsson, Dr. Isaac Skog, Prof. Fredrik Gustafsson and Mia Veibäck have been greatly appreciated. Any remaining mistakes are my own. I wish to thank all my co-authors as well as project collaborators. Also, thanks are in order to Dr. Gustaf Hendeby, Dr. Henrik Tidefelt and Dr. Jonas Linder for providing the $\mathrm{LT}_{\mathrm{EX}} \mathrm{X}$ class that has been used to write the thesis. Further, I am grateful for the financial support given by Vinnova Industry Excellence Center LINK-SIC. A special thanks goes to Prof. Simon Maskell for suggesting the title of the thesis.

The time at Automatic Control would have been less fantastic if it were not for all the amazing colleagues, many of whom I have come to call my friends. The absence of lower and upper limits on the level of discussions in the pub, in the fika room and on trips has been very entertaining and rewarding (not to mention scary at times).

When life throws you challenges you learn the value of having good friends and family to cheer you up, cheer you on and share a beer. I am so grateful for all of your support over the years.

Mia, Sam and Stina, your love and support mean the world to me and keep me focused on what is important in life. I am truly blessed!

Linköping, October 2018

Clas Veibäck 

$\begin{array}{ll}\text { Notation } & \text { XV }\end{array}$

1 Introduction 1

$1.1 \quad$ Target Tracking . . . . . . . . . . . . . . . . . . . 3

1.1.1 Pre-Processing . . . . . . . . . . . . . . 6

1.1.2 Association .................. 6

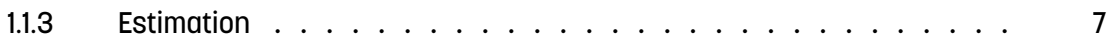

1.1 .4 Track Management . . . . . . . . . . . . . . . . 7

1.1.5 Presentation ..................... 8

1.2 Contributions and Publications . . . . . . . . . . . . . 8

1.3 Thesis Outline . . . . . . . . . . . . . . . . . . . . 12

2 Background $\quad 15$

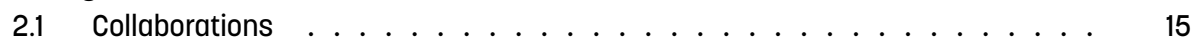

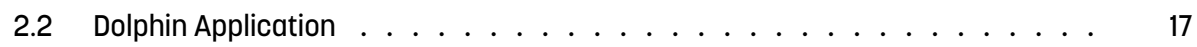

2.3 Bird Application . . . . . . . . . . . . . . . . . . . . . . . . . . . . . . . . . . . . . 19

2.4 Savannah Application . . . . . . . . . . . . . . . . . . . . . 20

2.5 Uncertain Time Scenarios . . . . . . . . . . . . . . . . . . . 23

2.6 Sea Ice Tracking Application . . . . . . . . . . . . . . . . . . . . . . . . 27

2.7 Gaussian Process Motion Model Scenarios . . . . . . . . . . . . . . . . . . 30

3 State-Space Models $\quad \mathbf{3 5}$

3.1 Discrete-Time State-Space Models . . . . . . . . . . . . . . . . . . . . 36

3.2 Continuous-Discrete State-Space Models . . . . . . . . . . . . . . . . . 37

3.3 Sensor Models . . . . . . . . . . . . . . . . . . . 39

3.4 Motion Models . . . . . . . . . . . . . . . . . . . . 40

3.5 Constrained Motion Model . . . . . . . . . . . . . . . . . . . . 42

3.5.1 Motivation . . . . . . . . . . . . . . . . . . . . . 42

3.5.2 Region Model . . . . . . . . . . . . . . . . . . . . . . . . . 44

3.5.3 Motion Model . . . . . . . . . . . . . . . . . . . . . . 45

3.6 Jump Markov Model . . . . . . . . . . . . . . . . . . . . . . . . . . . . . . . . 49

3.6.1 General Model . . . . . . . . . . . . . . . . 49 
3.6.2 Model Extension . . . . . . . . . . . . . . . . . . . . 51

3.7 Extended Target Model . . . . . . . . . . . . . . . . . . . . . 51

3.8 Uncertain Timestamp Model . . . . . . . . . . . . . . . . . . 53

3.9 Gaussian Process Motion Model . . . . . . . . . . . . . . . . . . . . . . . 57

3.9.1 Dynamic System with Unknown Influence . . . . . . . . . . . . . . 57

3.9.2 Approximation of Influence Function . . . . . . . . . . . . . . . . . . 57

3.9.3 Time-Varying Influence . . . . . . . . . . . . . . . . 59

3.9.4 Multiple Systems with Shared Influence . . . . . . . . . . . . . . 59

3.9.5 State-Dependent Influence . . . . . . . . . . . . . . . . . 60

3.9.6 Covariance Function for Influence . . . . . . . . . . . . . . 60

4 Camera Sensors $\quad 63$

4.1 Camera Model . . . . . . . . . . . . . . . . . . . . 63

4.2 Stationary Camera Model . . . . . . . . . . . . . . . . . 65

4.3 Foreground Segmentation . . . . . . . . . . . . . . . . . 69

4.3.1 Gaussian Mixture Model . . . . . . . . . . . . . . . . . 70

4.3.2 Moving Quantile Model . . . . . . . . . . . . . . . . . 73

4.3.3 Methods for Nonstationary Cameras . . . . . . . . . . . . . . 74

4.4 Data Reduction . . . . . . . . . . . . . . . . . . . . . . . . 77

5 Estimation $\quad \mathbf{8 1}$

5.1 Bayesian Solution . . . . . . . . . . . . . . . . . . . . 81

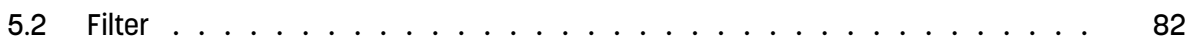

$5.2 .1 \quad$ Kalman Filter . . . . . . . . . . . . . . . . . . . . 82

$5.2 .2 \quad$ Extended Kalman Filter . . . . . . . . . . . . . . . . . . . . 84

5.2.3 Iterated Extended Kalman Filter . . . . . . . . . . . . . . . . . . . . . . . . . . . 85

5.2 .4 Particle Filter . . . . . . . . . . . . . . . . . . 85

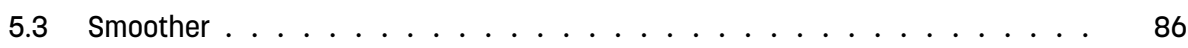

5.3.1 Linear Gaussian Smoothing . . . . . . . . . . . . . . . . . . 86

5.3 .2 State Injection . . . . . . . . . . . . . . . . . 88

5.3.3 Update of Posterior Distribution . . . . . . . . . . . . . . . 89

5.4 Jump Markov Model Filter . . . . . . . . . . . . . . . . . . . . . . . . . . . . . 91

5.4 .1 General Model . . . . . . . . . . . . . . . . . . . 91

5.4 .2 Model Extension . . . . . . . . . . . . . . . . . . . 9 93

5.5 Extended Target Model Filter . . . . . . . . . . . . . . . . . . . . . . . . . 99

5.6 Uncertain Timestamp Model Smoother . . . . . . . . . . . . . . . . . . . . . . . 102

$5.6 .1 \quad$ Posterior Distributions . . . . . . . . . . . . . . . . . . . 102

5.6 .2 Point Estimators _. . . . . . . . . . . . . . . . . 104

5.6.3 Approximate Computational Methods . . . . . . . . . . . 115

5.7 Gaussian Process Motion Model Filter . . . . . . . . . . . . . . . . . . . 123

5.7.1 Posterior Smoothing Distribution . . . . . . . . . . . . . 123

5.7.2 Recursive Estimation Using Extended Kalman Filter . . . . . . . . . . . 123

5.7.3 Implementation Considerations ............... 126

6 Data Association 133

6.1 Data Association Uncertainty . . . . . . . . . . . . . . . . . . 134 
6.1.1 Uncertainty Models . . . . . . . . . . . . . . . . . . . . 134

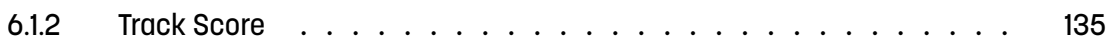

6.1.3 Track Quality . . . . . . . . . . . . . . . . . . . . 136

6.1.4 Linear Assignment Problem . . . . . . . . . . . . . . . . . . . 137

6.1.5 Coarse Gating and Clustering . . . . . . . . . . . . . . . . . . . 139

6.2 Multiple Hypothesis Tracker . . . . . . . . . . . . . . . . . . . . . . . . 140

6.3 Probabilistic Data Association Filter . . . . . . . . . . . . . . . . . 143

6.3.1 Standard Probabilistic Data Association Filter . . . . . . . . . . 143

6.3.2 Track Management . . . . . . . . . . . . . . . . . . . . 144

6.3.3 Modified Probabilistic Data Association Filter . . . . . . . . . . . . . 145

6.4 Labelled Multi-Bernoulli Filter . . . . . . . . . . . . . . . . . . . . . . . . 150

6.4.1 Labelled Multi-Bernoulli Filter . . . . . . . . . . . . . . . . . 150

6.4.2 Adaptive Birth Model . . . . . . . . . . . . . . . . . . . . . . . 154

6.4.3 Reformulation . . . . . . . . . . . . . . . . 154

7 Conclusions and Future Work 159

$\begin{array}{lll}\text { A Proofs } & 161\end{array}$

A.1 Integrated Weights . . . . . . . . . . . . . . . . . . . . . 161

A.2 State Injection . . . . . . . . . . . . . . . . . . . . . . . 162

A.3 Smoother Update . . . . . . . . . . . . . . . . . . . . . 166

A.4 Uniform Ellipse . . . . . . . . . . . . . . . . . 168

$\begin{array}{ll}\text { Bibliography } & 171\end{array}$ 

Mathematical Style

\begin{tabular}{cl}
\hline Notation & Meaning \\
\hline$a$ & Scalar variable or parameter \\
$\mathbf{a}$ & Vector variable or parameter \\
A & Matrix variable or parameter \\
$\mathcal{A}$ & Set variable or parameter \\
\hline
\end{tabular}

\section{Vector and Matrix Notation}

\begin{tabular}{cl}
\hline Notation & Meaning \\
\hline $\mathbf{I}_{a}$ & Identity matrix of dimension $a \times a$ \\
$\mathbf{I}$ & Identity matrix of applicable dimension \\
$\mathbf{0}$ & Vector or matrix of zeroes of applicable dimension \\
$\mathbf{A} \otimes \mathbf{B}$ & Kronecker product of matrices A and $\mathbf{B}$ \\
$\mathbf{a}^{\top}$ or $\mathbf{A}^{\top}$ & Transpose of vector a or matrix $\mathbf{A}$ \\
$\mathbf{a}_{\perp}$ & $\pi / 2$ rad clockwise rotation of two-dimensional vec- \\
& tor $\mathbf{a}, \mathbf{a}_{\perp}=\left(\begin{array}{l}a \\
b\end{array}\right)_{\perp}=(b)$ \\
$|\mathbf{A}|$ & Determinant of matrix $\mathbf{A}$ \\
$\operatorname{tr}(\mathbf{A})$ & Trace of matrix $\mathbf{A}$ \\
$\mathbf{a} \cdot \mathbf{b}$ & Scalar product of column vectors $\mathbf{a}$ and $\mathbf{b}, \mathbf{a}^{\top} \mathbf{b}$ \\
$\|\mathbf{a}\|$ & Norm of column vector $\mathbf{a}, \sqrt{\mathbf{a}^{\top} \mathbf{a}}$ \\
$\|\mathbf{a}\|_{\mathbf{A}}$ & Mahalanobis distance of vector $\mathbf{a}$, where $\mathbf{A}$ is positive \\
& definite, $\sqrt{\mathbf{a}^{\top} \mathbf{A}^{-1} \mathbf{a}}$ \\
$\tilde{\mathbf{a}}$ & Transforms a vector a to homogeneous coordinates, \\
& $\left(\mathbf{a}^{\top}, 1\right)^{\top}$ \\
$\mathbf{A}_{a, b}$ or $\mathbf{A}_{a b}$ & Element at row $a$ and column $b$ in matrix $\mathbf{A}$ \\
$a_{x}$ and $a_{y}$ & First and second element, respectively, of vector a \\
$\operatorname{diag}\left(a_{1}, \ldots, a_{b}\right)$ & Diagonal matrix with diagonal $a_{1}, \ldots, a_{b}$ \\
\hline
\end{tabular}




\section{Probability Notation}

\begin{tabular}{cl}
\hline Notation & Meaning \\
\hline$p_{\mathbf{a}}(\mathbf{a})$ or $p(\mathbf{a})$ & PDF or PMF of stochastic variable or expression a \\
$p(\mathbf{a} \mid \mathbf{b})$ & Conditional PDF or PMF of stochastic variable or ex- \\
& pression a given $\mathbf{b}$ \\
$\mathbf{a} \sim p(\mathbf{a})$ & Stochastic variable a follows distribution $p(\mathbf{a})$ \\
$\mathbf{E}(\mathbf{a})$ & Expected value of stochastic expression a \\
$\mathbf{C o v}(\mathbf{a})$ & Covariance of stochastic expression a \\
$\mathcal{N}(\boldsymbol{\mu}, \boldsymbol{\Sigma})$ & Multivariate normal distribution with mean $\boldsymbol{\mu}$ and \\
$\mathcal{N}(\mathbf{a} \mid \boldsymbol{\mu}, \boldsymbol{\Sigma})$ & Muvariance $\boldsymbol{\Sigma}$ \\
$\mathcal{I} \mathcal{W}(\nu, \mathbf{V})$ & Inverse Wishart distribution with $v \in \mathbb{R}^{+}$degrees of \\
$\mathcal{I} \mathcal{W}(\mathbf{A} \mid v, \mathbf{V})$ & Inveedom and matrix $\mathbf{V} \in S_{++}$ \\
$\mathcal{P} \mathcal{S}(\lambda)$ & Poisson distribution with intensity $\lambda$ \\
$\mathcal{P} \mathcal{S}(a \mid \lambda)$ & Poisson PMF of scalar $a$ \\
$\mathcal{U}(\mathcal{V})$ & Uniform distribution over set $\mathcal{V}$ \\
$\mathcal{U}(\mathbf{a} \mid \mathcal{V})$ & Uniform PDF of vector a \\
\hline
\end{tabular}

Set Notation

\begin{tabular}{cl}
\hline Notation & Meaning \\
\hline $\mathbb{N}$ & Set of natural numbers \\
$\mathbb{R}$ & Set of real numbers \\
$\mathbb{R}^{+}$ & Set of positive real numbers \\
{$[a, b]$} & Set of all real numbers in interval, $\{c \in \mathbb{R} \mid a \leq c \leq b\}$ \\
$a: b$ or $\{a: b\}$ & Set of all integers in an interval, $\{c \in \mathbb{Z} \mid a \leq c \leq b\}$ \\
$\mathbb{R}^{a}$ & Set of real column vectors of dimension $a$ \\
$\mathbb{R}^{a \times b}$ & Set of real matrices of dimension $a \times b$ \\
$S_{+}$ & Set of all positive semi-definite matrices \\
$S_{+}^{a}$ & Set of all $a \times a$ positive semi-definite matrices \\
$S_{++}$ & Set of all positive definite matrices \\
$S_{++}^{a}$ & Set of all $a \times a$ positive definite matrices \\
$S O(3)$ & Set of $3 \times 3$ orthogonal matrices with unit determi- \\
& nant \\
$\{\mathbf{a} \mid \operatorname{condition}\}$ & Set of all vectors a such that condition holds \\
$\left\{\mathbf{a}_{b}\right\}_{b \in \mathcal{A}}$ & Set of all vectors $\mathbf{a}_{b}$ such that $b \in \mathcal{A}$ \\
$\left\{\mathbf{a}_{b}\right\}_{b=c}^{d}$ & Set of all vectors $\mathbf{a}_{b}$ such that $b \in\{c: d\}$ \\
$|\mathcal{A}|$ & Cardinality of set $\mathcal{A}$ \\
$\mathcal{A} \cup \mathcal{B}$ & Union of sets $\mathcal{A}$ and $\mathcal{B}$ \\
$\mathcal{A} \cap \mathcal{B}$ & Intersection of sets $\mathcal{A}$ and $\mathcal{B}$ \\
$\mathcal{A} \backslash \mathcal{B}$ & Difference between sets $\mathcal{A}$ and $\mathcal{B}$ \\
$1_{\mathcal{A}}(\mathcal{B})$ & Inclusion function, 1 if $\mathcal{B} \subseteq \mathcal{A}$ and 0 otherwise \\
$\mathcal{F}(\mathcal{A})$ & Collection of all subsets of set $\mathcal{A}$ \\
\hline &
\end{tabular}




\section{Other Notation}

\begin{tabular}{|c|c|}
\hline Notation & Meaning \\
\hline $\arctan 2(a, b)$ & $\begin{array}{l}\text { Arctangent with two arguments, numerator } a \text { and } \\
\text { denominator } b \text {, returning output in the correct quad- } \\
\text { rant }\end{array}$ \\
\hline $\log (a)$ & Natural logarithm of $a$ \\
\hline$a \propto b$ & $\begin{array}{l}\text { Expressions } a \text { and } b \text { are proportional, } \exists c \in \mathbb{R}^{+} \text {such } \\
\text { that } a=c b\end{array}$ \\
\hline$\dot{\mathbf{a}}$ or $\dot{\mathbf{a}}(t)$ & Derivative of $\mathbf{a}(t)$ with regards to time $t, \frac{d \mathbf{a}}{d t}(t)$ \\
\hline $\mathbf{a}^{+}$or $\mathbf{A}^{+}$ & Denotes an update of a vector a or matrix $\mathbf{A}$ \\
\hline$\lceil a\rceil$ & Ceiling of $a$ \\
\hline$a !$ & Factorial of $a$ \\
\hline$\langle a, b\rangle$ & $\begin{array}{l}\text { Inner product of functions } a(c) \text { and } b(c) \text {, } \\
\int a(c) b(c) d c\end{array}$ \\
\hline$\delta_{a}(b)$ & Kronecker delta function, 1 if $a=b$ and 0 otherwise \\
\hline$\delta_{\mathbf{a}}(\mathbf{b})$ & Dirac delta function \\
\hline
\end{tabular}

Abbreviations

\begin{tabular}{cl}
\hline Abbreviation & Meaning \\
\hline ANEES & Average Normalized Estimation Error Squared \\
AWGNSSM & Additive White Gaussian Noise State-Space Model \\
CMM & Constrained Motion Model \\
CTM & Coordinated Turn Model \\
EKF & Extended Kalman Filter \\
EM & Expectation-Maximization \\
ETT & Extended Target Tracking \\
FIC & Fully Independent Conditional \\
FISST & Finite Set Statistics \\
GLMB & Generalized Labelled Multi-Bernoulli \\
GNN & Global Nearest Neighbour \\
GNSS & Global Navigation Satellite System \\
GP & Gaussian Process \\
GPMM & Gaussian Process Motion Model \\
IEKF & Iterated Extended Kalman Filter \\
IMM & Interacting Multiple Models \\
JMAP & Joint Maximum A Posteriori \\
JMM & Jump Markov Model \\
JPDA & Joint Probabilistic Data Association \\
KF & Kalman Filter \\
LGSSM & Linear Gaussian State-Space Model \\
LMB & Labelled Multi-Bernoulli \\
MAP & Maximum A Posteriori \\
MHT & Multiple Hypothesis Tracker \\
\hline &
\end{tabular}




\begin{tabular}{cl}
\hline Abbreviation & Meaning \\
\hline MMSE & Minimum Mean Squared Error \\
MTT & Multiple Target Tracking \\
NCPM & Nearly Constant Position Model \\
NCVM & Nearly Constant Velocity Model \\
NCAM & Nearly Constant Acceleration Model \\
NSSM & Nonlinear State-Space Model \\
NN & Nearest Neighbour \\
OOS & Out Of Sequence \\
PDA & Probabilistic Data Association \\
PDF & Probability Density Function \\
PEM & Prediction Error Method \\
PF & Particle Filter \\
PMF & Probability Mass Function \\
PMHT & Probabilistic Multiple Hypothesis Tracker \\
PHD & Probability Hypothesis Density \\
PSSM & Probabilistic State-Space Model \\
RFS & Random Finite Set \\
RMSE & Root Mean Squared Error \\
RTSS & Rauch-Tung-Striebel Smoother \\
SAR & Synthetic Aperture Radar \\
SDE & Stochastic Differential Equation \\
SOR & Subset of Regressors \\
SSM & State-Space Model \\
SPRT & Sequential Probability Ratio Test \\
STT & Single Target Tracking \\
TBD & Track Before Detect \\
TRI & Terrestrial Radar Interferometer \\
UTM & Uncertain Timestamp Model \\
UAS & Unmanned Aerial System \\
UKF & Unscented Kalman Filter \\
\hline & \\
&
\end{tabular}




\section{1}

\section{Introduction}

Target tracking is a mature area of research, which traditionally has had a strong focus on military and aviation applications. With the advent of cheap and commercially available sensors and increased computational resources, see Figure 1.1 for an example, new possibilities have opened up for target tracking in a broader group of applications. New applications pose new challenges that need to be addressed. As an illustration of such an application a scenario is shown in Figure 1.2 where a radar is deployed on the savannah to track rhinos. The main contributions of the thesis revolve around modelling and estimation challenges encountered in target tracking applications. A range of applications found in nature and simulations are used to exemplify the functionality of the proposed methods. A few established methods for target tracking are presented and a number of specific challenges are covered in more detail. Solutions to five commonly encountered problems, corresponding to the contributions, are proposed.

Problem I concerns learning a complete or partial motion model while tracking targets. Part of the dynamics is modelled as a Gaussian process (GP), which can be learned online. Many methods exist for identifying systems [115], and a recent development is to model systems using GPs [67]. The method proposed in this thesis employs a sparse GP to allow for sequential estimation with constant memory and computational complexity.

Problem II is about observations that suffer from uncertainty in the sampling times. The problem of uncertain sampling time has received little attention in the scientific literature, with out-of-sequence (oos) measurements [10, 16] and time delays [90,123] being notable exceptions. A linear Gaussian state-space model (LGSSM) is considered in this thesis for multiple observations with uncertain sampling times.

Problem III concerns targets that have to obey motion constraints that are not easily incorporated into the standard state-space model (sSM). Conventional mo- 


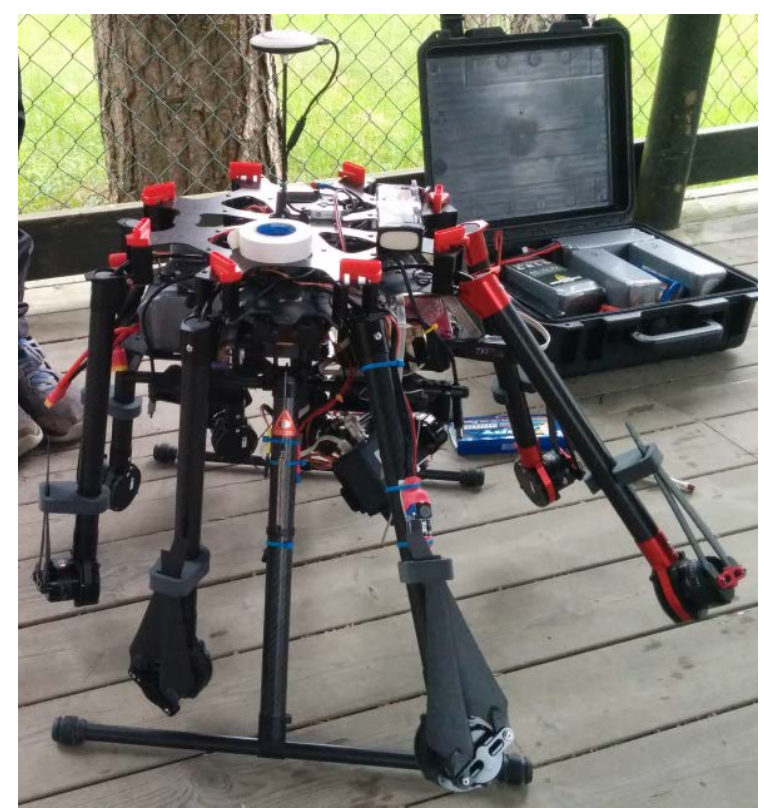

Figure 1.1: Quadcopters are versatile platforms that can be equipped with a number of sensors and deployed in a range of tracking applications. The uncertainty in position of the platform results in particular challenges in tracking applications.

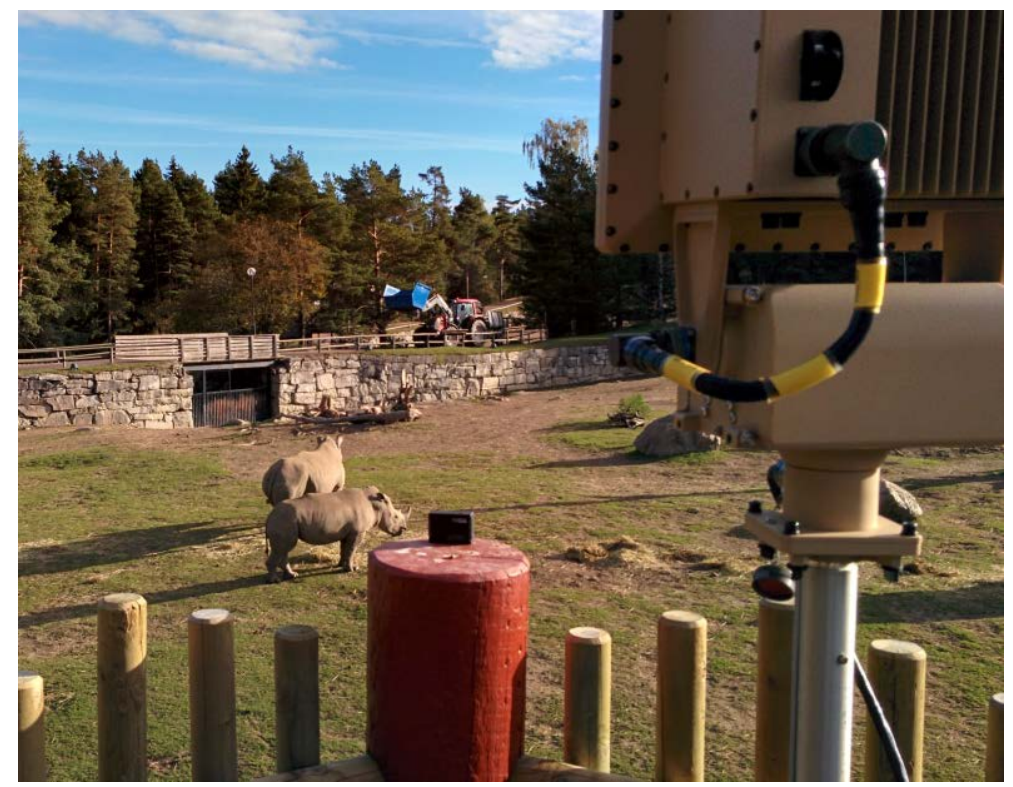

Figure 1.2: A Doppler radar deployed on the savannah at Kolmairden Wildlife Park. The radar can track large animals as well as vehicles and persons. 
tion models are assumed to be unconstrained in the real coordinate space and do not enforce such motion constraints, which results in infeasible predictions. The particular case considered is a target that is constrained to be within a region, see Figure 1.3 for an example of such a situation. One option is to project the state back into the region. Another approach is considered in this thesis where the motion model is adapted to the region to produce feasible predictions.

Problem IV is to incorporate measurements of the mode when estimating a jump Markov model (JMM). In traditional JMMs the SSM parameters depend on the mode, resulting in an indirect effect on the measurement. The case considered in this thesis is that in addition to state-dependent measurements there are also observations of an arbitrary distribution that directly depends on the mode.

Problem V deals with achieving computational performance in demanding data association algorithms. A reformulation of the labelled multi-Bernoulli (LMB) filter is proposed that exposes a structure that is exploited to construct an efficient implementation of the algorithm. Modern tracking algorithms are important in noisy applications with a large number of targets, such as in the sea ice tracking example in Figure 1.4.

The concept of target tracking is introduced in Section 1.1 as a framework for solutions. Contributions and publications by the author are discussed in Section 1.2 and the outline of the thesis is presented in Section 1.3.

\subsection{Target Tracking}

Target tracking is the problem of estimating the position, and possibly more characteristics, of a target or targets from sensor data. This involves all or a subset of the following steps [31]:

- pre-processing of sensor data to obtain detections;

- association of detections over time to form tracks;

- estimation of a target state for each track using associated detections;

- track management; and

- presentation of the result to the end user.

A track is defined as a sequence of detections that are generated by the same target according to an association hypothesis. Usually, the track is represented by its posterior distribution given models for the sensors and the motion, rather than the list of associated detections. A track is normally classified into various categories and its quality is estimated to evaluate its status as representing a true target. A target is an object of interest that can be detected by a sensor. An association hypothesis assigns each detection to a track or classifies it as noise. Each hypothesis therefore consists of a number of tracks corresponding to hypothesized true targets. 


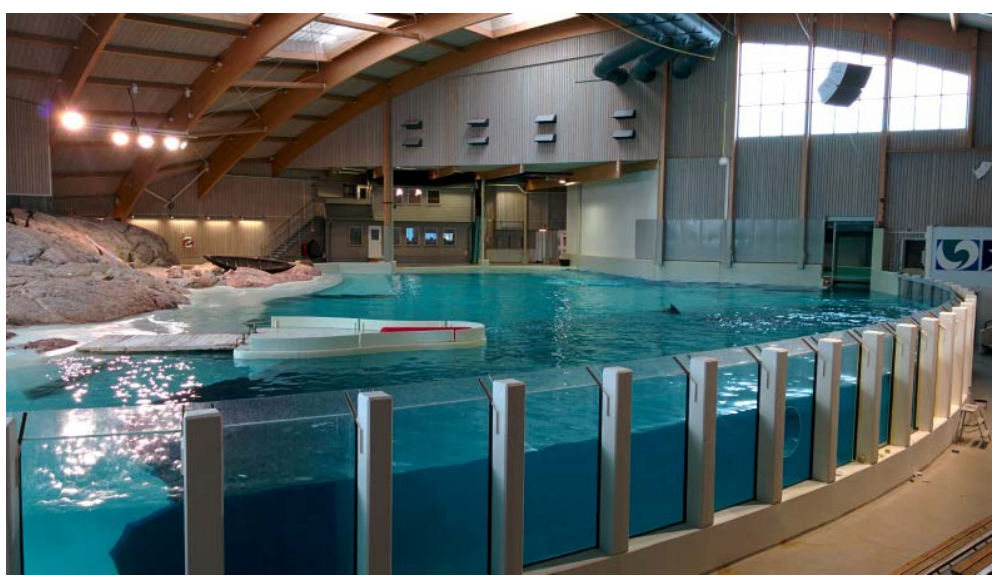

Figure 1.3: The dolphinarium at Kolmairden Wildlife Park with a camera mounted in the ceiling for tracking. The dolphins are confined to the basin, which results in constraints in the model that can be used to improve the predictions of their movements.

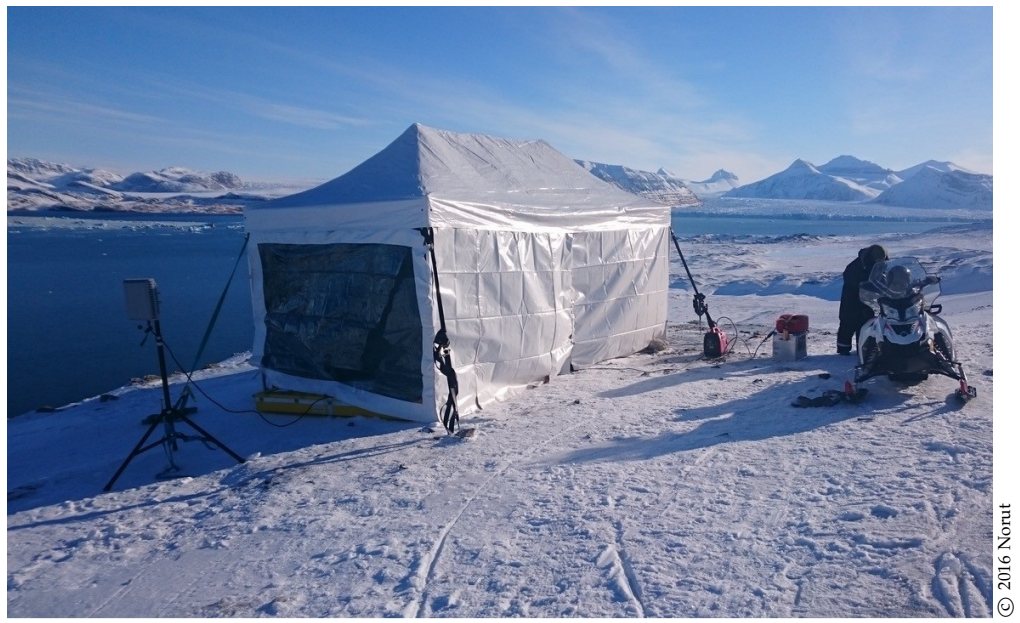

Figure 1.4: A radar deployed in a fjord on Svalbard to track sea ice. The ice might be stationary, drift independently as small ice objects or move in large groups, requiring a combination of various tracking solutions. Figure courtesy of Norut. 


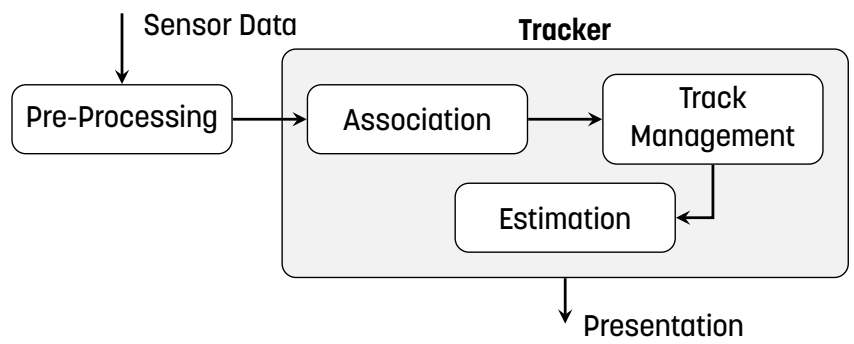

Figure 1.5: Elements of a single hypothesis tracker for multiple target tracking.

Remark 1.1: There are no clear definitions or unique names for many of the concepts within the field, e.g., the definition of tracks are rarely explicit, and target tracking, sensor fusion, inference and estimation can often be used interchangeably as well as measurement, detection and observation. Often the meaning is given by the context, avoiding the need for a definition. This thesis is no different, but to avoid confusion, definitions will be given at times.

A target tracking framework can be represented as a pipeline of modules with well-defined interfaces, as shown in Figure 1.5. The modules have access to a central collection of tracks and hypotheses. Often there is overlap between the modules, so a more flexible interface is needed in reality, but the pipeline is useful as an illustration of the framework.

The design workflow of a target tracking framework involves deciding on algorithms for each of the steps outlined in the beginning of the section. However, preceding the selection of algorithms, the targets and sensors are modelled. The types of models determine the possible algorithms and allow for making informed design decisions.

Two common assumptions in target tracking are that a detection is generated by at most one target and that a target can generate at most one detection [31]. If the former is violated additional logic is required and if the latter is violated extended target tracking (ETT) [107] algorithms are needed. A nuisance in tracking applications is that false detections that are not generated by true targets occur, commonly known as clutter [11]. In addition, true targets possibly do not generate a detection at every time step. This is known as a missed detection.

Applications may consider multiple targets, referred to in the literature as multiple target tracking (МтT), while in other applications it is known a priori that only a single target is present, referred to as single target tracking (STT) [11]. If only a single target is present and there are no false detections, the data association and track management steps are redundant, since only a single track is needed. In all other cases the uncertainty in data association between detections and tracks needs to be considered. There is an abundance of literature on the topic of target tracking, see e.g. [11, 12, 17, 30,121]. An overview of established methods and common issues in target tracking is given in [31].

An overview of each module in the pipeline is given next. There are also other possible solutions to consider, such as track-before-detect (TBD) [8] that per- 
forms tracking directly on raw data, or association-free algorithms, such as the probability hypothesis density (PHD) filter [186].

\subsubsection{Pre-Processing}

The pre-processing step involves all activities required to obtain detections that can be used for association and estimation. The activities depend on the type of sensor that is used. To extract detections from camera sensors, simple thresholding might suffice in one application, while state-of-the-art computer vision methods might be required in other applications [75]. A few such methods are described in Chapter 4. For radars, low-level signal processing is often used together with a threshold to produce detections [31]. Thresholds, or similar tuning parameters, define a trade-off between false and missed detections. The current set of tracks can be used to adaptively set the thresholds, improving the performance of the algorithm.

\subsubsection{Association}

Given a set of detections, this step aims to find the best associations between detections and tracks. An ambiguity in association results in multiple possible hypotheses. Simple methods, such as nearest neighbour (NN) [11] and probabilistic data association (PDA) [11, 31], that work well for STT basically select the most likely detections for each track. More advanced methods, such as global nearest neighbour (GNN) [31] and joint probabilistic data association (JPDA) [11, 31], designed for MTT globally find the overall most likely associations for tracks and detections, resulting in a single hypothesis. A complete solution would consider all possible associations in different hypotheses and present the currently most likely hypothesis. One such mTt solution is the multiple hypothesis tracker (МНт) [31, 153]. However, maintaining all hypotheses over time is computationally demanding, so clever approximations are used that only maintain a set of the most likely hypotheses.

Each hypothesis generated in the association step consists of a set of tracks where the associations with detections are assumed to be given and estimation can be performed. In addition to associating detections to existing tracks, they may also be identified as false detections or new tracks, requiring additional logic. To improve performance and to avoid unlikely associations a gate is used for each track to only consider detections that are likely. The methods are described in depth in [31] and the references therein. A few aspects and methods for data association are covered in Chapter 6.

In the last decade a new association framework has emerged where the multiobject state is represented by a generalized labelled multi-Bernoulli (GLMB) random finite set (RFS) [189]. It is shown to result in an analytic solution to the multi-object filtering problem, denoted the GLMB filter. The foundation of the filter is theoretically different from conventional target tracking algorithms, but they share many features on the implementation level [197]. A single hypothesis version of the filter, denoted the Lмв filter, was proposed in [154] and is described further 
in Chapter 6. Like conventional state-of-the-art target tracking algorithms, these are often computationally demanding, so clever approximations and efficient implementations are often a necessity to achieve good computational performance.

\subsubsection{Estimation}

Given a set of detections associated over time to the same track in a single hypothesis, the current state of the track is estimated. There is an abundance of literature on this topic that goes beyond the problem of target tracking, see e.g. $[5,79,81,103,159]$.

The main idea is to use an SSM to describe the dynamics of the target and model the sensors, where the states are estimated using measurements from the sensors. Estimators for ssMs are commonly divided into:

- filters, where measurements are available up to the current time;

- predictors, where measurements are available up to a previous time; and

- smoothers, where measurements are available up to a future time.

The most notable method is the Kalman filter (KF) [101], which for the LGSSM gives the exact Bayesian solution in closed form. A corresponding smoother algorithm is the Rauch-Tung-Striebel smoother (RTSS) [152]. For nonlinear or nonGaussian models an approximation, such as the extended Kalman filter (EKF) [164] or unscented Kalman filter (UKF) [98, 100], can be used or a sequential Monte Carlo method, such as the particle filter (PF) [76]. If the target type is unknown or the target behaviour is expected to change, a JMM [175] is suitable, for which a set of filters or smoothers can be used to estimate the mode. Common estimation methods for such models are the filter bank with pruning [7] and the interacting multiple models (IмM) filter [32]. Chapter 3 covers ssms and Chapter 5 covers methods for state estimation.

The ssm is often chosen from a set of conventional models [114]. However, an unconventional model tailored to the application can improve the performance and may be a necessity for the algorithm to perform satisfactorily in certain cases. Motion characteristics that do not match the model are referred to as manoeuvres.

\subsubsection{Track Management}

Track management is performed to initiate, merge and terminate tracks. For MHT also hypothesis management is considered. Unassociated detections are used to initiate new tracks, called initiators or tentative tracks. A track is confirmed if a sufficient number of measurements have been associated with the initiator, determined by a track score or initiator logic. Methods can also perform two layers of data association, first for confirmed tracks and then for initiators, to guarantee that confirmed tracks are given higher priority. A track is deleted if an insufficient number of detections have occurred, determined by the track score 
or a deletion logic. For low-resolution sensors, entire groups of targets might belong to a single track, and additional logic is often required to handle merging and splitting of such tracks when targets enter or leave the group. In hypothesisoriented MHT, initiators are treated differently, where management is performed on the hypothesis level. It is intractable to consider all possible hypotheses, so the set of most likely hypotheses is maintained between iterations, and unlikely hypotheses are pruned. The methods are described in detail in [31] and a few track management policies are covered in Chapter 6.

\subsubsection{Presentation}

Presentation of results in target tracking is often very important but non-trivial and highly dependent on the application. One solution is to display the current position of the tracks in the most likely hypothesis on a map. For MHT often more advanced methods are required to summarize the hypotheses [31, 50]. Additionally, operators might be interested in more information about the targets, such as trajectories, velocities, cardinalities, predicted positions, sizes or types.

In extreme applications tracks might have to be summarized for display to not overwhelm the operator. For tracking applications without real-time constraints, a summary of statistics or events might suffice for presentation. In automated surveillance systems often a warning for anomalous behaviour is most interesting to operators. Presentation of tracking results is not explicitly considered in this thesis.

\subsection{Contributions and Publications}

There are five main contributions in this thesis:

I. the development of a Gaussian process motion model (GPMM) [184];

II. the development and analysis of an uncertain timestamp model (UTM) [182, 183];

III. the development of a constrained motion model (смM) [181];

IV. the development of an extension to the JMM, which incorporates state-independent measurements [27]; and

V. the application of a reformulation of an equation in the LMB filter [141].

All contributions concern tracking of objects over time using inexact sensors. Through modification of established methods and models the aim is to improve tracking performance in various scenarios, such as scenarios suffering from object occlusions, movement constraints, environmental disturbances or uncertain time information. 
Licentiate's Thesis Contributions III and IV and partly Contribution II have already been published in the author's licentiate's thesis,

C. Veibäck. Tracking of Animals Using Airborne Cameras. Licentiate's Thesis No. 1761, Linköping University, Nov. 2016.

Consequently, the contents of the two theses partially overlap.

Contribution I This contribution has been submitted and is under review for publication,

C. Veibäck, J. Olofsson, T. R. Lauknes, and G. Hendeby. Learning target dynamics while tracking using Gaussian processes. IEEE Transactions on Aerospace and Electronic Systems, submitted June 13, 2018. ISSN 0018-9251,

in IEEE Transactions on Aerospace and Electronic Systems. All examples in this thesis concerning the sea ice learning application and the velocity field scenario are also presented in the paper. The paper presents methods for:

- modelling target dynamics using a GP, described in Section 3.9; and

- estimation and learning in a GPMM, described in Section 5.7.

The author's contribution to the paper was in theoretical derivations, implementation of algorithms and writing the manuscript. The co-authors aided plenty in discussions and feedback as well as providing data for the paper.

Contribution II This contribution has been submitted and accepted for publication,

C. Veibäck, G. Hendeby, and F. Gustafsson. Uncertain timestamps in linear state estimation. IEEE Transactions on Aerospace and Electronic Systems, accepted for publication August 21, 2018. ISSN 0018-9251. doi:10.1109/TAES.2018.2869648. (c) 2018 IEEE,

in IEEE Transactions on Aerospace and Electronic Systems. The majority of the examples in this thesis concerning uncertain time are also presented in the paper. The paper presents methods for:

- modelling observations that are sampled with uncertain timestamps, described in Section 3.8; and

- estimation in a UTM, described in Section 5.6.

The content is reused in this thesis courtesy of IEEE. The author's contribution to the paper was in theoretical derivations, implementation of algorithms and writing the majority of the manuscript. The co-authors aided plenty in discussions and feedback as well as in part writing the manuscript. The contribution is based on a publication, 
C. Veibäck, G. Hendeby, and F. Gustafsson. On fusion of sensor measurements and observation with uncertain timestamp for target tracking. In Proceedings of the International Conference on Information Fusion, pages 1268-1275, Heidelberg, Germany, July 2016. (C) 2016 IEEE,

on models with a single observation sampled at an uncertain time. All examples in this thesis concerning the simple uncertain time are also presented in the paper. The paper presents methods for:

- modelling a single observation sampled at an uncertain time, partly described in Section 3.8; and

- estimation in a UTM with a single observation sampled at an uncertain time, partly described in Section 5.6.

The content is reused in this thesis courtesy of IEEE. The author's contribution to the paper was in theoretical derivations, implementation of algorithms and writing the majority of the manuscript. The co-authors aided plenty in discussions and feedback as well as in part writing the manuscript.

Contribution III This contribution has been published in a paper,

C. Veibäck, G. Hendeby, and F. Gustafsson. Tracking of dolphins in a basin using a constrained motion model. In Proceedings of the International Conference on Information Fusion, pages 1330-1337, Washington D. C., USA, July 2015. (C) 2015 IEEE,

on a tracking solution for dolphins in captivity. All examples in this thesis concerning the dolphin application are also presented in the paper. The paper presents methods for:

- modelling constrained motion, described in Section 3.5;

- modelling stationary camera sensors, described in Section 4.2;

- segmenting foreground in video, described in Section 4.3;

- reducing detection data, described in Section 4.4; and

- data association, described in Section 6.3.3.

The content is reused in this thesis courtesy of IEEE. The author's contribution to the paper was in theoretical derivations, implementation of algorithms and writing the majority of the manuscript. The co-authors aided plenty in discussions and feedback as well as in part writing the manuscript. 
Contribution IV This contribution has in part been published in a paper,

G. Bianco, M. Ilieva, C. Veibäck, K. Öfjäll, A. Gadomska, G. Hendeby, M. Felsberg, F. Gustafsson, and S. Åkesson. Emlen-funnel experiments revisited: methods update for studying compass orientation in songbirds. Ecology and Evolution, 6(19):6930-6942, Sept. 2016. ISSN 2045-7758. doi:10.1002/ece3.2383. (c) 2016 The Authors. Ecology and Evolution published by John Wiley \& Sons Ltd,

on updated methods for studying compass orientation in songbirds. The majority of the examples in this thesis concerning the bird application are also presented in the paper. The appendix of the paper presents methods for:

- extending the JMм to incorporate state-independent measurements, described in Section 3.6.2; and

- estimation in the Jмм extended with state-independent measurements, described in Section 5.4.2;

The author's contribution to the paper was in deriving and implementing the filter in the Computer vision method, providing results from the method to the first author and in writing the parts of the manuscript and appendix related to the filter.

Contribution V The contribution has been published in a paper,

J. Olofsson, C. Veibäck, and G. Hendeby. Sea ice tracking with a spatially indexed labeled multi-Bernoulli filter. In Proceedings of the International Conference on Information Fusion, pages 376-383, Xi'an, China, July 2017. doi:10.23919/ICIF.2017.80096720. (C) 2017 IEEE,

on tracking sea ice using an LMB filter. All examples in this thesis concerning the sea ice tracking application are also presented in the paper. The paper presents:

- a method for detecting ice objects in radar data, described in Section 4.3;

- a reformulation of the LMB filter, described in Section 6.4.3; and

- a method for gating using spatial indexing, described in Section 6.1.5.

The content is reused in this thesis courtesy of IEEE. J. Olofsson initiated the project and lead the research and writing of the manuscript in collaboration with the author of this thesis. The contribution of the author of this thesis to the paper involves development and implementation of the pre-processing algorithm, tuning of the filter parameters and writing associated parts of the manuscript. The other co-authors aided plenty in discussions and feedback. 
Excluded Related Publications The author has published two additional papers that are related to the sea ice tracking application, but which are not included in this thesis. The first paper,

J. Olofsson, A. Lindahl Flåten, and C. Veibäck. Gaussian field current estimation from drift sea ice tracking with the labeled multi-Bernoulli filter. In Proceedings of the OCEANS Conference, Anchorage, AK, USA, Sept. 2017,

extends the results in [141] to also estimate the velocity of the currents using a Gaussian field. The author's contribution to the paper was in the reuse of the results from the original paper and in discussions on the proposed ideas with the first author. The second is a paper,

J. Olofsson, C. Veibäck, G. Hendeby, and T. A. Johansen. Outline of a system for integrated adaptive ice tracking and multi-agent path planning. In Proceedings of the Workshop on Research, Education and Development of Unmanned Aerial Systems, Linköping, Sweden, Oct. 2017. doi:10.1109/RED-UAS.2017.8101636,

on a proposed framework for tracking sea ice using various sensors. The author's contribution to the paper was in writing the first draft on situation maps and motion planning and in discussions on the content with the co-authors.

Unrelated Publications The author has also published two papers that are not directly related to the content of this thesis. The first paper,

F. Ceragioli, G. Lindmark, C. Veibäck, N. Wahlström, M. Lindfors, and C. Altafini. A bounded confidence model that preserves the signs of the opinions. In Proceedings of the European Control Conference, pages 543548, Aalborg, Denmark, June 2016. doi:10.1109/ECC.2016.78103410,

presents a bounded confidence model. The author's contribution to the paper was in initial derivations and simulations for the model, performed as an exercise in a course on the subject by Prof. Claudio Altafini. The second paper,

S. Gunnarsson, Y. Jung, C. Veibäck, and T. Glad. IO (implement and operate) first - an alternative way to approach the automatic control subject. In Proceedings of Utvecklingskonferensen för Sveriges ingenjörsutbildningar, pages 163-167, Uppsala, Sweden, Feb. 2016,

concerns teaching in industrial control systems. The author's contribution to the paper was in discussions on the content with the first author of the paper after having taught in the course.

\subsection{Thesis Outline}

The disposition of the of the thesis follows that of the natural design workflow for a target tracking framework. The thesis contains five applications and three 
simulated scenarios that will be used throughout the thesis to evaluate, verify, exemplify and show applicability of the theory. Solutions for the applications are developed as relevant theory is covered.

In Chapter 1, the concept of target tracking and its challenges are introduced. Publications and contributions of the thesis are also presented.

In Chapter 2, background, motivation, collaborators and related work for the research are presented. The chapter also introduces the applications and simulated scenarios.

In Chapter 3, the concepts of continuous- and discrete-time ssms are introduced. The chapter describes several conventional motion models that are common in target tracking as well as basic measurement models. Five models that are tailored to particular problems are also presented.

Chapter 4 covers methods for modelling camera sensors and image processing. Cameras are used as sensors in three of the applications in this thesis. A significant amount of pre-processing is often required and the nonlinearities of the sensors are in many cases non-trivial. Another application uses a radar as a sensor, which is pre-processed using the same techniques.

Chapter 5 describes a selection of methods for estimation. Estimation concerns inferring information from available measurements using a model of the system, which for an SSM is referred to as state estimation. The chapter also considers methods for estimating and learning other unknown parameters.

In Chapter 6, methods for managing uncertainty in observation origins are presented. This is a common nuisance in target tracking and is considered in three of the applications. Estimation in such scenarios often leads to an intractable problem. The chapter describes several approximate solutions and heuristics that can be applied to make the problem tractable and achieve reasonable computational performance.

Chapter 7 concludes the thesis and discusses future work. The appendix presents proofs to four propositions used in the thesis. 



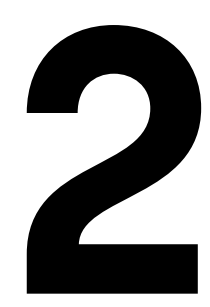

\section{Background}

Background, motivation and related work for the research are presented in this chapter and the applications are introduced. The latter are used to evaluate, verify, exemplify and show applicability of the theory. Table 2.1 is provided as a reference for the features of each application. The research has been conducted with a number of collaborators, presented in Section 2.1, that have provided motivation, data and input. Note that the backgrounds to the research introduced in the application sections focus mainly on related work on similar applications, while the backgrounds to the research introduced in the scenario sections are more extensive and focus mainly on related theoretic work.

\subsection{Collaborations}

Biologists spend a significant part of their time collecting and analysing animal data. The manual methods they use are prone to bias and the time could perhaps be better spent on other research activities. Target tracking can provide a solution to this problem by automating the procedures used to collect and analyse data. The first project is a collaboration with biologists at Kolmården Wildlife Park interested in studying the behaviour of dolphins. This research is introduced in Section 2.2 and resulted in Contribution III. The second project is a collaboration with biologists at Lund University interested in studying the behaviour of birds. This research is introduced in Section 2.3 and resulted in Contribution IV.

Many endangered animal populations in the world are still on the decline. There are numerous underlying causes for this, but poaching is a significant reason in many wildlife reserves. Park rangers often have scarce resources and would benefit from cost-effective solutions providing situational awareness, which would allow for improved coordination of conservation efforts. This 
Table 2.1: List of theory and features that are exemplified by the different applications.

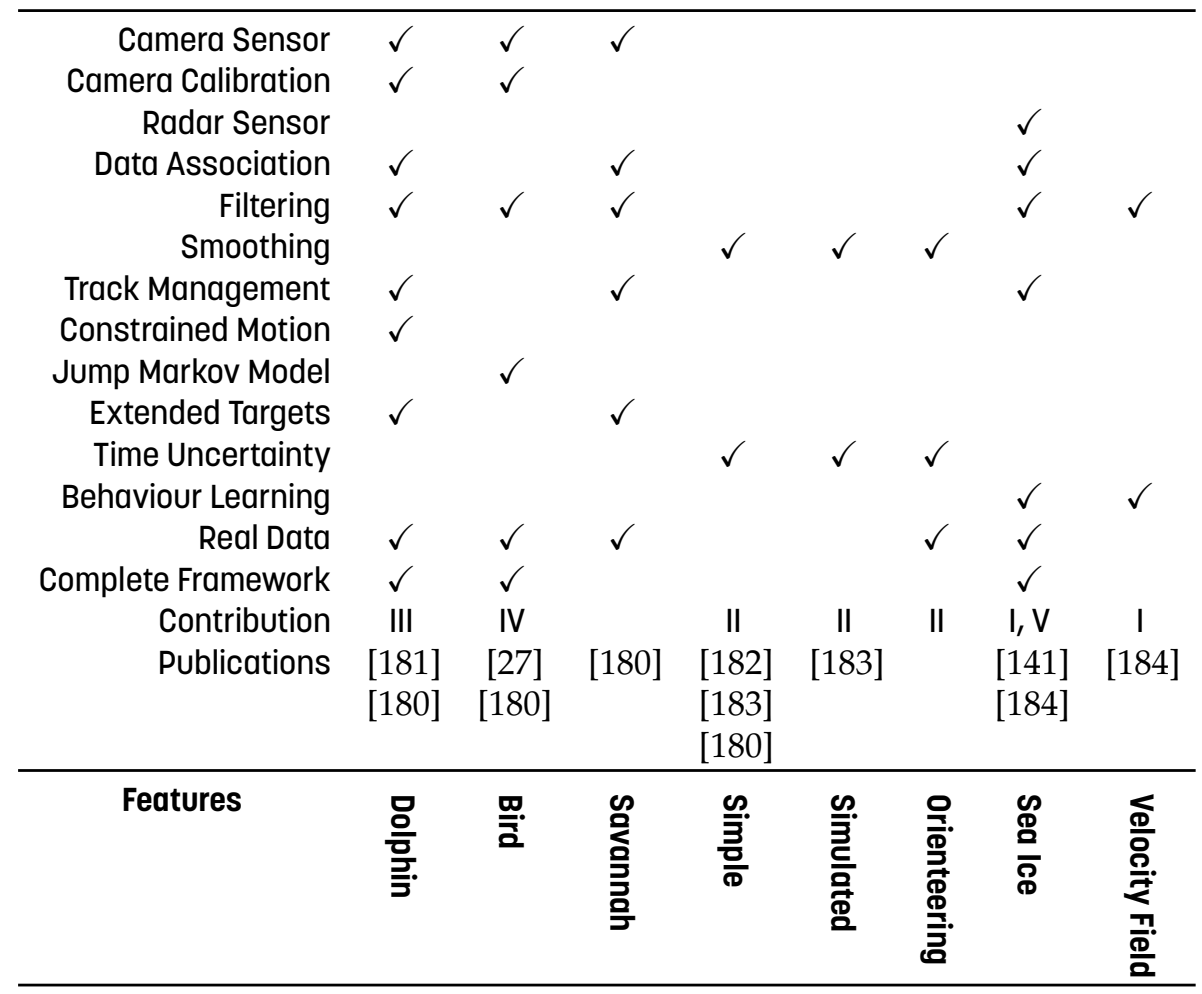

could be provided by incorporating various sensors, such as ground and airborne cameras, smart phones and radars, into a single network for tracking animals. The third project is a collaboration with partners in the Smart Savannahs [162] project. One application involves tracking animals using electrooptical and infrared cameras mounted on a drone. This is introduced in Section 2.4. Research in this project also involves observations with uncertain timestamps, which is introduced in Section 2.5 and resulted in Contribution II.

Work by the maritime industry in the polar regions of the planet is unavoidably linked with hazards such as drift ice. Increased presence fuelled by economic interests in the Arctic, has for several decades [150] called for research in the field of ice management. While managing ice is of great importance to polar ventures, predicting ice movement has proven difficult [58], concluding that observations are essential for tracking. The fourth project is a collaboration with maritime researchers NTNU and Norut researching sea ice tracking. One application involves tracking sea ice from radar data gathered on Svalbard using a state-of-the-art tracker. This is introduced in Section 2.6 and resulted in Contribution V. Research in this project also involves the development of a model for the online estimation of currents influencing the targets. This is introduced in 
Section 2.7 and resulted in Contribution I.

Providing biologists, park rangers and the maritime industry with complete solutions for the problems they face is a rather ambitious goal. Instead, steps on the way are presented in the form of methods to approach particular problems within target tracking. Solutions for a few applications are presented with clear ambitions towards aiding biologists, park rangers and the maritime industry. However, the main interest is in the technical challenges posed by the problems within the applications and the technical solutions to those problems.

\subsection{Dolphin Application}

A recent project for tracking dolphins in a dolphinarium, described in [102], was set up at Brookfield Zoo in Chicago. The researchers utilized multiple overhead and underwater cameras to record data for over a year. Due to the massive amounts of data, manual tracking was only attempted for a small portion of the data and from a single view. The paper describes a method of using robust principal component analysis [40] and Gaussian-mixture background modelling [65] to detect the dolphins and a compressive tracker [200] to extract trajectories. The trajectories were compared to the manually annotated data with promising results.

There have also been attempts to track dolphins in the wild to study their behaviour, e.g. in [66], where boat-side overhead cameras were used, and in [88], where underwater cameras and hydrophones were used together. Another project related to this research is described in [33], where a large number of underwater cameras over the duration of three years recorded fish at a coral reef. The purpose was, however, to classify the species of the fish rather than to track their location, but it demonstrates the growing importance of camera surveillance in animal behaviour studies.

Application 2.1 introduces a scenario where biologists want to track dolphins in captivity to observe their behaviour. This application illustrates Contribution III, presented in Section 3.5, which is a motion model tailored to targets in captivity. The proposed solution for this application and related background theory were published by the author in [181, (C) 2015 IEEE].

\section{Application 2.1: Tracking of Dolphins}

Dolphins swimming in a basin are tracked in this challenging application using a fisheye camera mounted in the ceiling. See Figure 2.1 for an illustration of available footage. The biological motivation [179] is to understand how the behavioural pattern is affected by underwater sonar transponders. A better understanding can be obtained with this research of how the dolphin's internal navigation system works. Today, tracking is performed by manually annotating the positions of the dolphins in the video.

The application is a classic target tracking problem, with false detections from pre-processing, individuals forming tight groups and a need to model the extension of targets. New challenges include shadows at the bottom of the basin and 


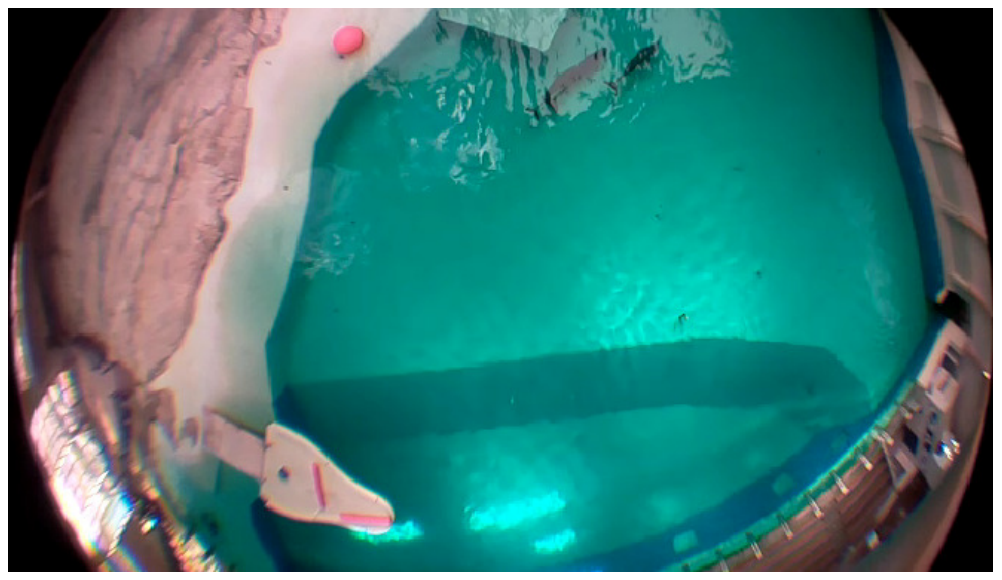

Figure 2.1: A single frame from a video recorded of the dolphins by the fisheye camera. A number of challenges inherent to this problem, e.g., lighting conditions and occlusions, are apparent in the figure.

sun light through the ceiling windows that causes large local changes in light conditions. The scene includes hard constraints caused by the basin, occlusion from a platform in the water and missed detections caused by a limited field of view from the fisheye camera. Another challenge is the difficulty to obtain sufficient data to calibrate the camera.

The goal of the application is to construct a complete target tracking solution from camera to trajectories. To achieve this goal methods for image processing, camera calibration, target tracking and modelling of dynamic systems are used. In addition, new methods for the complete solution are required for:

- using more information from background estimation for tracking;

- modelling a camera without the possibility of a proper calibration;

- data reduction and mapping of a large number of detections;

- modelling dolphin motion in captivity; and

- object extension estimation.

The data is generously provided by Kolmården Wildlife Park. The solution is presented throughout the thesis, where all application descriptions in the thesis with the word dolphin in the caption are related to this application. The models are developed in Applications 3.2 and 3.3, the pre-processing is described in Applications 4.2, 4.3 and 4.8 and the full framework, the results and an analysis of the filter are presented in Applications 6.2 and 6.3. 


\subsection{Bird Application}

Bird migration has fascinated biologists for centuries. The birds follow a migratory program inherited from their parents during their first migration. The program encodes durations and directions to fly, and to navigate the birds rely on biological compasses based on the sun, stars and magnetic fields [85]. A method for studying this behaviour, in which migratory birds are kept in funnel-shaped cages, was introduced half a century ago in [61]. The cages, called Emlen funnels, record scratches from the birds with ink or thermal paper as they attempt to take off and allow biologists to study the compass mechanisms of migratory birds. Several results from studies involving Emlen funnels have been published, e.g. in $[60,198]$.

The Emlen funnel method is primarily manual and has remained largely unchanged in the last few decades. Attempts at automating the process using electronic switches to detect movement, e.g. in [194], have shown not to improve the method and in addition suffered interference from electromagnetic noise. However, passive cameras mounted at a distance from the birds, used in [131], do not cause such interferences. Recently, software has also been made available, presented in [132], for evaluating activity and orientations of the birds, showing a trend to modernize the traditional method.

Application 2.2 introduces a scenario where biologists want to track the movements of a bird in an Emlen funnel [61] to observe its behaviour. This application illustrates Contribution IV, presented in Section 3.6.2, through a novel use of measurements extracted through image processing to determine the behaviour of a target. The proposed solution for this application was briefly described by the author in [27, (c) 2016 The Authors. Ecology and Evolution published by John Wiley \& Sons Ltd].

\section{Application 2.2: Tracking of Birds}

An application is considered where the times and directions of takeoffs and the amount of activity of the European robin is studied. For such experiments, the birds are placed in Emlen funnels [61] to record their movements. Traditionally, the walls are lined with scratch-sensitive paper where the scratches are counted manually after a certain time. The amount of activity and the average orientation of takeoffs are then estimated. The procedure of counting is often time-consuming, tedious and sensitive to the bias of the individuals performing the counting.

A proposed development of this method is to install camera surveillance of the birds during the experiments. See Figure 2.2 for an illustration of the available footage. There are mainly three advantages to this approach. Firstly, it allows for automation of the procedure, both saving time and reducing errors. Secondly, a temporal dimension is added to the data that is not available with the traditional method. Thirdly, experiments can be conducted for longer time periods. Further development of the method could potentially reduce the stress on the bird by allowing for larger cages during the experiments.

The goal is to estimate the activity and the takeoff times and directions of 


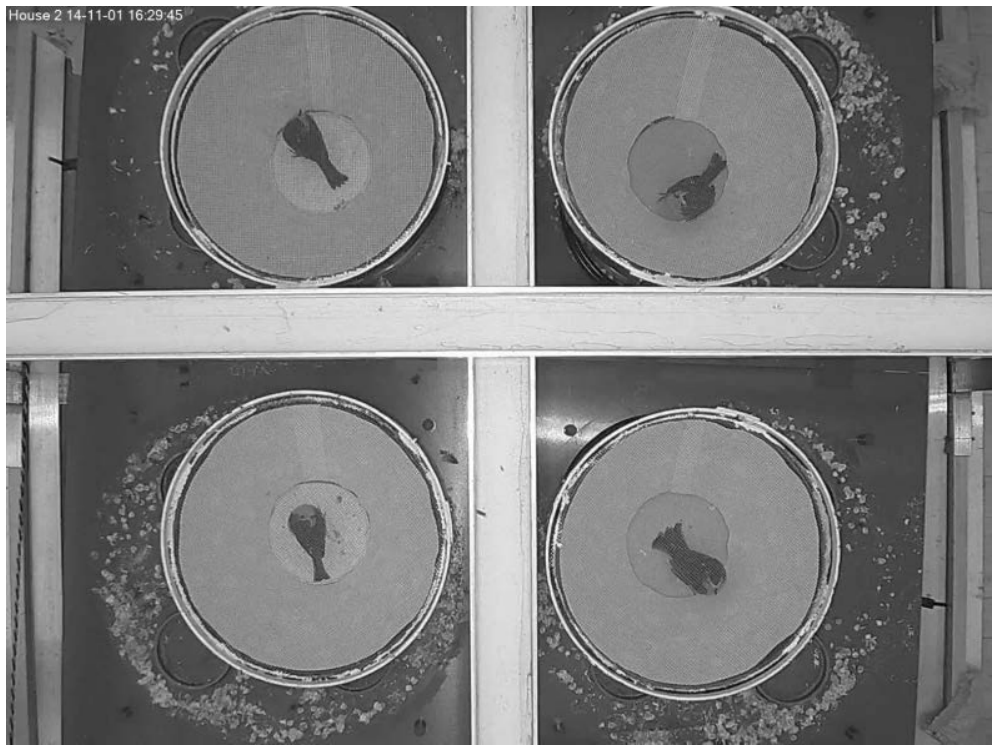

Figure 2.2: A single frame from a video recorded of the songbirds by a camera.

birds from a recorded video. To achieve this goal methods for camera calibration, JMMs and estimation for such models are used. In addition, new methods for the complete solution are required for:

- modelling a camera without the possibility of a proper calibration;

- modelling modes in a JMM;

- estimating times of takeoffs; and

- estimating direction of takeoffs.

The data is generously provided by the Centre for Animal Movement Research at Lund University. The solution is presented throughout the thesis, where all application descriptions with the word bird in the caption are related to this application. The motion and sensor models are developed in Applications 3.4 and 4.1, the pre-processing is described in Application 4.5, and the results of the solution are presented in Application 5.1.

\subsection{Savannah Application}

In the last few decades, the market for commercially available low-cost small unmanned aerial systems (UAS) has exploded. Flight time is still rather limited and in most cases significant technical skills are required to set up and operate the UASs. However, they provide new opportunities for solving traditionally tedious, 
difficult or dangerous problems. One promising area for deploying uAss is in surveillance of large land masses, such as wildlife reserves, to count and track wild animals for conservation purposes. Wildlife is traditionally monitored by foot, satellite or manned aircraft, with high costs as a result.

As a first attempt, many projects using UAss essentially put a camera in the sky, allowing conservationists to count and track animals manually in the video, see e.g. [93, 96, 185] for a small selection of many such projects. Other projects are more ambitious and in addition to deploying uAss perform image processing for automatic detection and counting of animals, see e.g. [74, 124, 178, 195] for a selection of such projects. A project targeted directly at anti-poaching of rhinoceros is presented in [133], where a UAS was deployed in the KwaZuluNatal province in South Africa. The article discusses the technical difficulties involved, advantages and disadvantages of the approach as well as the ethical and legal aspects. A project for tracking animals in captivity from an aerial cableway through Kolmården Wildlife Park is presented in [62]. The long time between detections seemed to be an issue for maintaining tracks.

Application 2.3 introduces a scenario where the aim is to track animals, particularly rhinoceros, in a wildlife reserve in Kenya. The application is in its current state not a complete framework, but serves as an opportunity to demonstrate state-of-the-art target tracking algorithms.

\section{Application 2.3: Tracking of Savannah Animals}

A scenario is considered in this application where animals on the savannah at Kolmården Wildlife Park are recorded from a quadcopter using a video camera and a thermal camera. Ultimately the goal is to classify the animals and track their absolute locations and extents in the real world. As a first attempt to find a solution, the application considers tracking the position and the extent of the animals in the image. The application also serves as a demonstration of a stateof-the-art ETT algorithm.

Single frames from the video camera and thermal camera are presented in Figures 2.3 and 2.4, respectively. The two main differences between the cameras are that the video camera has a much higher resolution and field of view, and that animals are more visible in the image from the thermal camera due to their high body temperatures. Especially compare the two lechwes that are clearly visible in the top right corner of Figure 2.4, but are barely visible in Figure 2.3.

The ultimate goal is to track the position and extension of savannah animals in real-world coordinates using an airborne camera as well as to classify the targets. As a stepping stone, a lower goal is set in this thesis to track the positions and extensions of the savannah animals in image coordinates. To achieve this, methods for background estimation and state-of-the-art ETT are used. The data was collected together with partners in the project Smart Savannahs [162]. The solution is presented throughout the thesis, where all application descriptions with the word savannah in the caption are related to this application. The models are presented in Application 3.6, the pre-processing is described in Application 4.6 and the results of the tracking solution are shown in Application 6.1. 


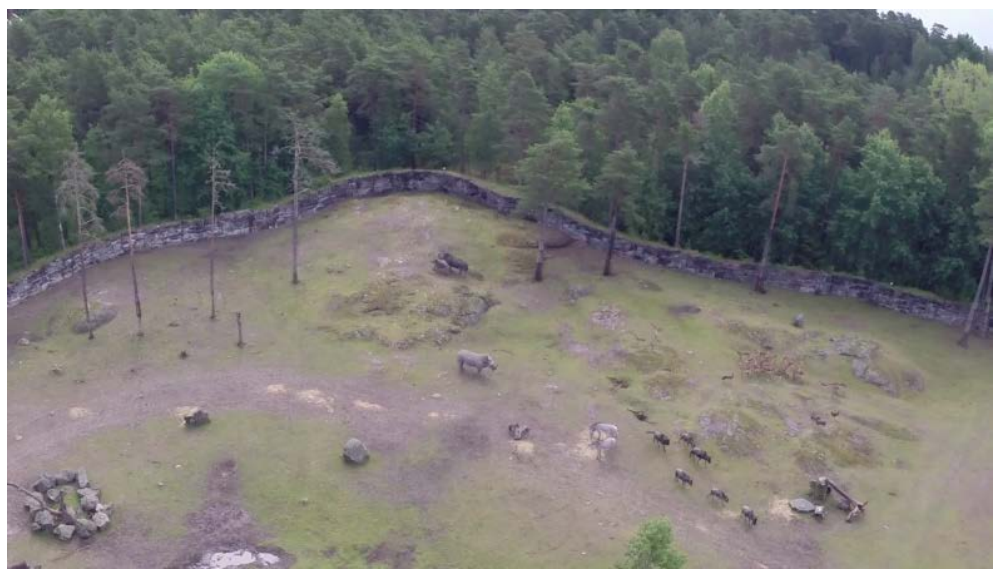

Figure 2.3: A frame recorded with a video camera over the savannah at Kolmairden Wildlife Park from a quadcopter.

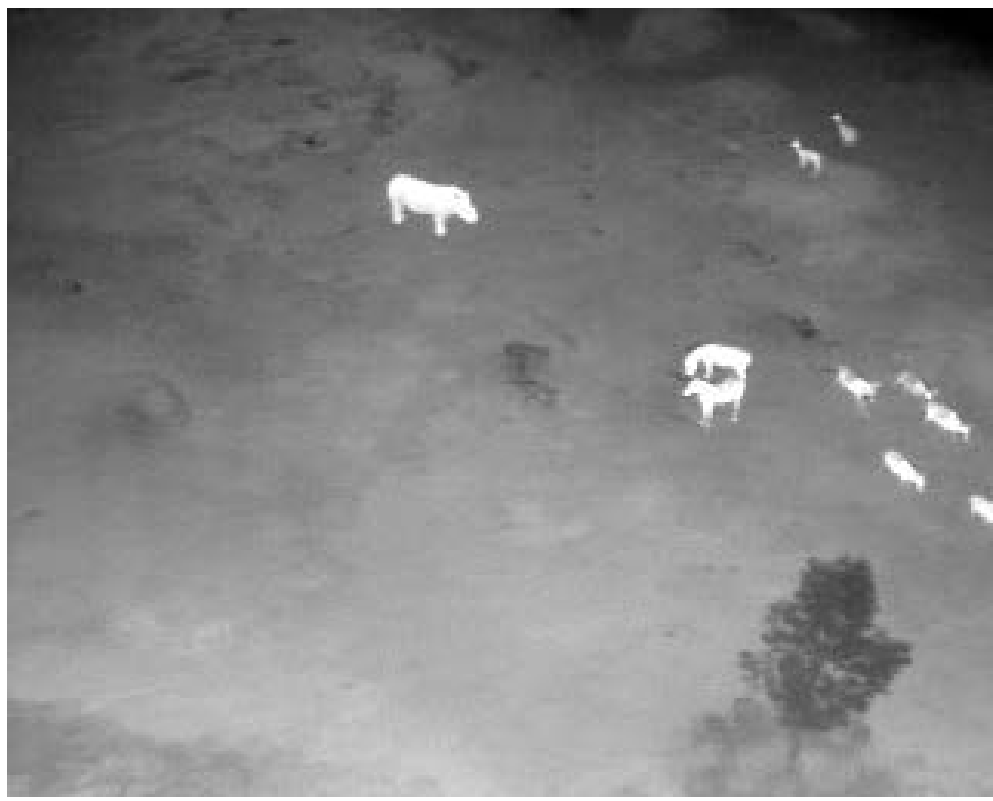

Figure 2.4: A frame recorded with a thermal camera over the savannah at Kolmairden Wildlife Park from a quadcopter. 


\subsection{Uncertain Time Scenarios}

A formulation of the linear state estimation problem that is useful at times is proposed with mixed measurements of two types:

M measurements with precise timestamps; and

o observations with uncertain timestamps.

The interest in this problem originally comes from an idea for fusion of radar and droppings. In the project Smart Savannahs [162], tracking algorithms are developed for following rhinoceroses inside a sanctuary. This can be done with sensors of type $\mathbf{M}$, complemented with manual observations of type $\mathrm{O}$ from the rangers. The rangers spend considerable time on patrol, recording indirect rhinoceros sightings, including droppings, foot prints, resting places and browsing damages. They are all of type o and are particularly useful in the labelling problem, i.e., which track belongs to which rhinoceros. Another application is in crime scene investigations. The place of the crime is often known precisely, but not the time, and witness statements often contain uncertainty in both time and place, while surveillance cameras are precise in time. A third application is to estimate a bias in the type $M$ measurements, when unbiased type o observations are available. The observations of type o need to be incorporated into a higher level data association algorithm, however, this thesis will concern state estimation given a hypothesis of association.

The proposed approach is based on extending the optimal Bayes filter to consider uncertain stochastic timestamps, which usually are considered to be known exactly. Given a prior on the timestamps, the smoothed posterior distribution of the states conditioned on observations of both types, $\mathrm{M}$ and $\mathrm{O}$, is derived. This leads to a posterior that can be expressed as a compound distribution. This distribution is often difficult to compute analytically. However, for discrete timestamps and an LGSSM the distribution can be computed exactly as a mixture of Gaussians, although the number of components may be prohibitively large. A sampling method is proposed to approximate the posterior in the general case and a few estimators are derived. The proposed sampling method computes the smoothing distribution for a large number of similar sets of timestamps. To improve the performance a method is derived to update an existing smoothing distribution in a single pass when inserting or removing an observation at an arbitrary point in time.

The problem considered bears a close resemblance to the problems of oos and time-delayed measurements, in particular when the time-delay is uncertain. In such problems, the time delay is often assumed to be bounded, and mainly filtering applications are considered. The problem of time delays has been treated extensively over the years, however, relatively little work has concerned timing uncertainty. In the literature nothing has been found that extends the localized time delay to directly consider arbitrary timestamps with large uncertainty intervals, which is considered in this thesis. For this reason, a slightly different formulation of the problem is chosen.

The problem of fusing time-delayed measurements with a known times- 
tamp can be solved using a Kalman filter with augmented states, first proposed in [43]. By augmenting the state vector with delayed state vectors, the covariance between a delayed measurement and the current state is implicitly computed, allowing for a correct measurement update. This naive approach is rather inefficient and various improved methods are proposed and discussed in $[99,111,177]$. The more complicated case when the measurements also might arrive out of sequence is treated in [10, 16, 90, 123]. An efficient implementation is proposed in [199], which is also extended to handle delays in continuous time. The effects of time delays on the performance of systems are well studied, see e.g. [135, 160, 161]. Detections occurring at random, but known, times are considered in [13,165]. A reverse problem is considered in [47] where the time uncertainty is estimated for a position given the posterior.

Further, random sampling times and delays have been considered [172], as well as methods to estimate unknown static or slowly varying delays [136, 137]. In [99] a few methods for managing discrete uncertain time delays in a filter are evaluated. The best performance is obtained by considering the problem as uncertain data association and using a PDA filter [31], which is similar to the minimum mean squared error (MMSE) estimator derived in this thesis. Worse performance is obtained using maximum likelihood association, which has similarities with the maximum a posteriori (MAP) estimator derived in this thesis. However, the PDA filter is expensive, so instead they propose an approximate method using covariance union to combine the state posterior distributions when updated using the minimum and maximum time delay, respectively. This seems to perform well when localized time delays are considered, but the approximation is less reasonable when considering arbitrary timestamps. It is also not clear how to extend this method to deal with multiple uncertain observations with overlapping uncertainty intervals.

An uncertain time delay problem where the true timestamp is intermittently available is considered in [130]. However, a measurement of the timestamp is obtained for each sample with additive Gaussian noise. The parameters of the noise are jointly estimated with the state in a filter. The proposed filter method is to approximate the augmented state posterior using a Monte Carlo method that sequentially samples from the timestamp posterior distribution. This bears a resemblance with the sampling approach proposed in this thesis, but the chosen sampling method requires that the order of the timestamps is known and does not directly extend to large or overlapping uncertainty intervals.

A modification of the probabilistic multiple hypothesis tracker (РМHT), where in addition to target data association uncertainty, uncertain time delays are considered, is proposed in [45]. The algorithm applies the expectation-maximization (EM) method [53] in a sliding window to obtain a filter estimate, initialized by the prediction of the estimate from the previous time step. A special case of this method is derived in this thesis to efficiently compute the MAP estimate of the states, modified to consider arbitrary timestamps.

Applications 2.4-2.6 stem from a problem of combining measurements from rhinoceros from traditional sensors, such as radars or cameras, with observations from park rangers, such as browsing damage or droppings caused by the 
rhinoceros. The former type of measurements might be uncertain in position, but has an accurate timestamp, while the latter type of observations might be accurate in position, but has an uncertain timestamp. All three applications illustrate Contribution II, presented in Section 3.8.

Application 2.4 introduces a scenario involving a simple one-dimensional ssM incorporating an observation with an uncertain timestamp. The purpose of this application is to provide a simple scenario for an analysis of the impact of the additional observation on the posterior distribution of the state and its estimators. The analysis and the derivations of the posterior distributions and estimators were published by the author in [182, (C) 2016 IEEE].

\section{Application 2.4: Simple Uncertain Time Scenario}

A simple scenario is considered in this application, where a trajectory in one dimension is measured to start at position $0 \mathrm{~km}$ at time $0 \mathrm{~h}$ and end in $1 \mathrm{~km}$ at time $1 \mathrm{~h}$. An observation at position $0.5 \mathrm{~km}$ is then given with a flat prior on the timestamp between $0 \mathrm{~h}$ and $1 \mathrm{~h}$ in time. See Figure 2.5 for an illustration of the scenario. One can of course argue that any physical quantity that starts at $0 \mathrm{~km}$ and ends at $1 \mathrm{~km}$ must pass through position $0.5 \mathrm{~km}$, so this observation seems to contain little information. This is not quite true, however, since the posterior distribution is shown to narrow down in an interesting way. The case of an observation at position $1.5 \mathrm{~km}$ is also considered with a much more narrow prior distribution.

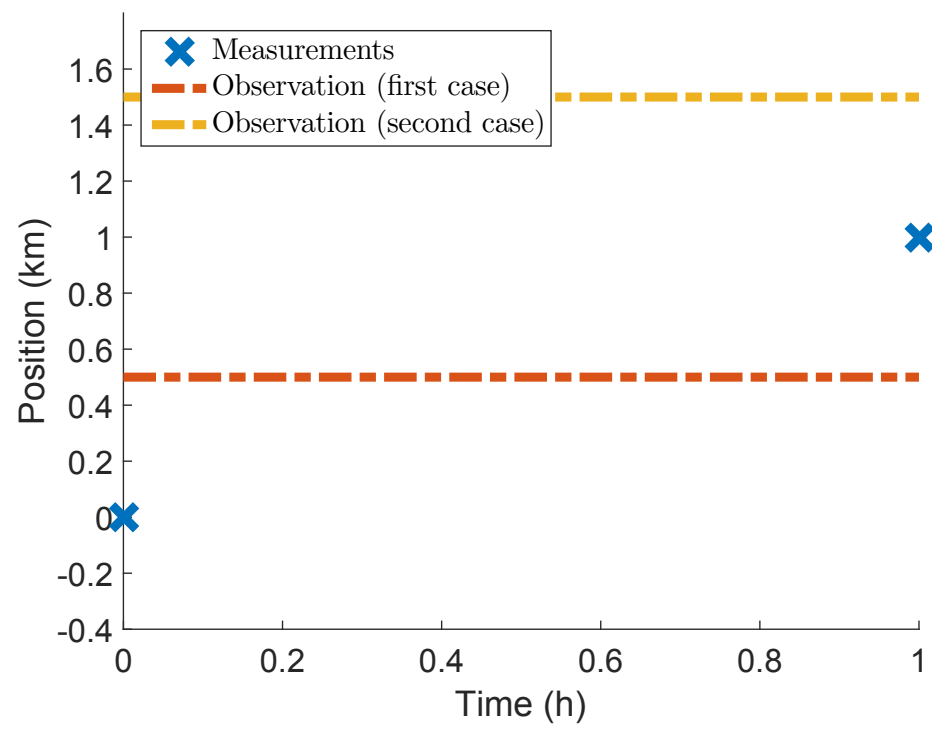

Figure 2.5: The measurements with precise timestamps and observations for the two cases considered in the simple uncertain time scenario, where the uncertainty in time is represented by the horizontal lines.

The goal is to obtain posterior distributions and estimators for a one-dimensional LGSSM with a single observation sampled at an uncertain time. To achieve 
this goal methods are used for smoothing in an SSM and for point estimation. In addition, new methods are required for

- modelling of an observation with an uncertain timestamp in an ssM; and

- computing smoothing posterior distributions for such models.

The solution is presented throughout the thesis, where all application descriptions with the word simple in the caption are related to this application. The model is presented in Application 3.7, the posterior is derived in Application 5.2 and a few estimators are proposed and analysed in Applications 5.3, 5.4, 5.6 and 5.7 .

Application 2.5 introduces a more complicated scenario involving a two-dimensional SSM incorporating two observations with uncertain timestamps. The purpose of this application is to provide a scenario for an analysis of the impact of multiple observations with uncertain timestamps on the posterior distribution of the state and its estimators. The analysis and the derivations of the posterior distributions and estimators are accepted for publication in IEEE Transactions on Aerospace and Electronic Systems [183, (C) 2018 IEEE].

\section{Application 2.5: Simulated Uncertain Time Scenario}

A simulated scenario is considered in this application, where a trajectory in two dimensions is simulated. Noisy measurements with precise timestamps are obtained for three segments of the trajectory and two less noisy observations with uncertain timestamps are obtained, one in each gap, between the segments. See Figure 2.6 for an illustration of the scenario, showing the true trajectory $\mathcal{X}$, the measurements $\mathcal{Y}$ and the observations $\mathcal{Z}$ with uncertain timestamps.

The goal is to obtain posterior distributions and estimators for a two-dimensional LGSSM with two observations sampled at uncertain times. To achieve this goal the same methods as for the simple uncertain time scenario are required. In addition, methods are required for approximate computation of the

- posterior distribution and MMSE estimator using Gibbs sampling [69, 71]; and

- MAP estimator using the EM algorithm [53].

The solution is presented throughout the thesis, where all application descriptions with the word simulated in the caption are related to this application. The model is presented in Application 3.8, the posterior and estimators for the application are presented in Application 5.8 and the results are further discussed in Application 5.9.

There is no data available for the original scenario with rhinoceros that motivated the model. Application 2.6 instead introduces a scenario with a corresponding problem where the aim is to improve the location estimate of an orienteering sprinter. 


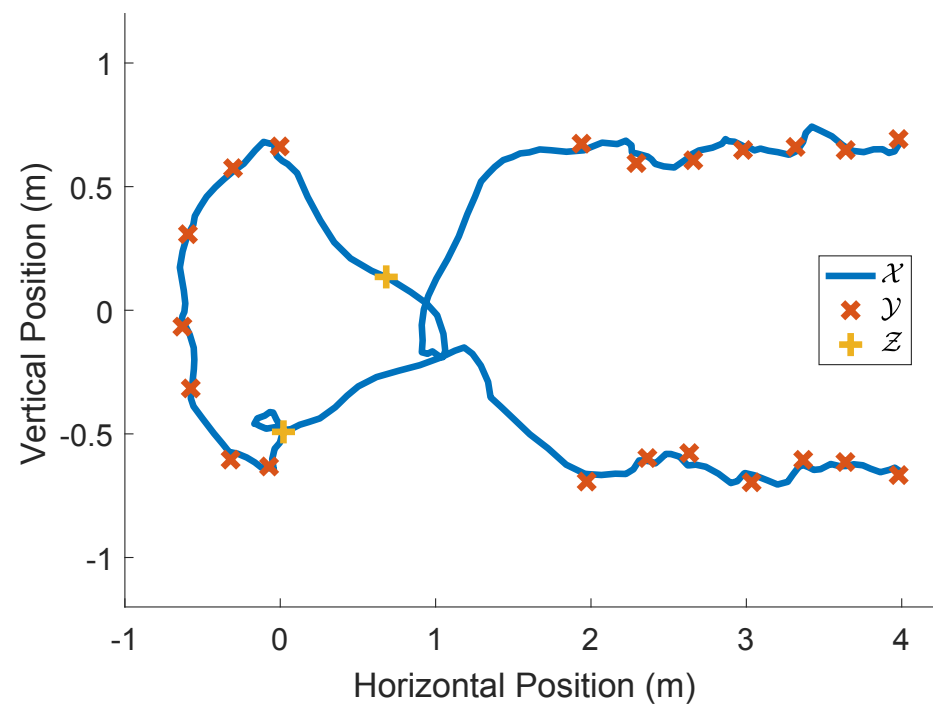

Figure 2.6: The simulated measurements $\mathcal{Y}$, observations $\mathcal{Z}$ and ground truth $\mathcal{X}$ for the scenario considered in the simulated uncertain time scenario.

\section{Application 2.6: Tracking of an Orienteering Sprinter}

An application is considered where a sprinter in orienteering is passing controls in a competition, as shown in Figure 2.7. The position of the sprinter is measured using a global navigation satellite system (GNSS). In parts of the race the accuracy of the GNSS is poor. However, the location of the controls are known with high accuracy and, since it is known that the sprinter passed the control, this information can be used to improve the estimated position of the sprinter.

The goal is to improve the location estimate of a sprinter using control points as observations with uncertain timestamps. To achieve this goal, the same methods as for the simulated uncertain time scenario are required. The data is generously provided by Oskar Ljungqvist and OK Orion. The solution is presented throughout the thesis, where all application descriptions with the word orienteering in the caption are related to this application. The model is presented in Application 3.9 and the posterior and estimators for the application are presented in Application 5.5.

\subsection{Sea Ice Tracking Application}

A comprehensive overview of ice management in practical use is provided in [57]. The field deals with the detection, tracking and forecast of ice, but also the physical actions taken to avoid collisions. For tracking the movement of sea ice, multiple sensors have been studied, such as satellite-carried synthetic aperture radar (SAR) [139], UASS [87, 95, 112] and, as studied in this thesis, terrestrial radar 


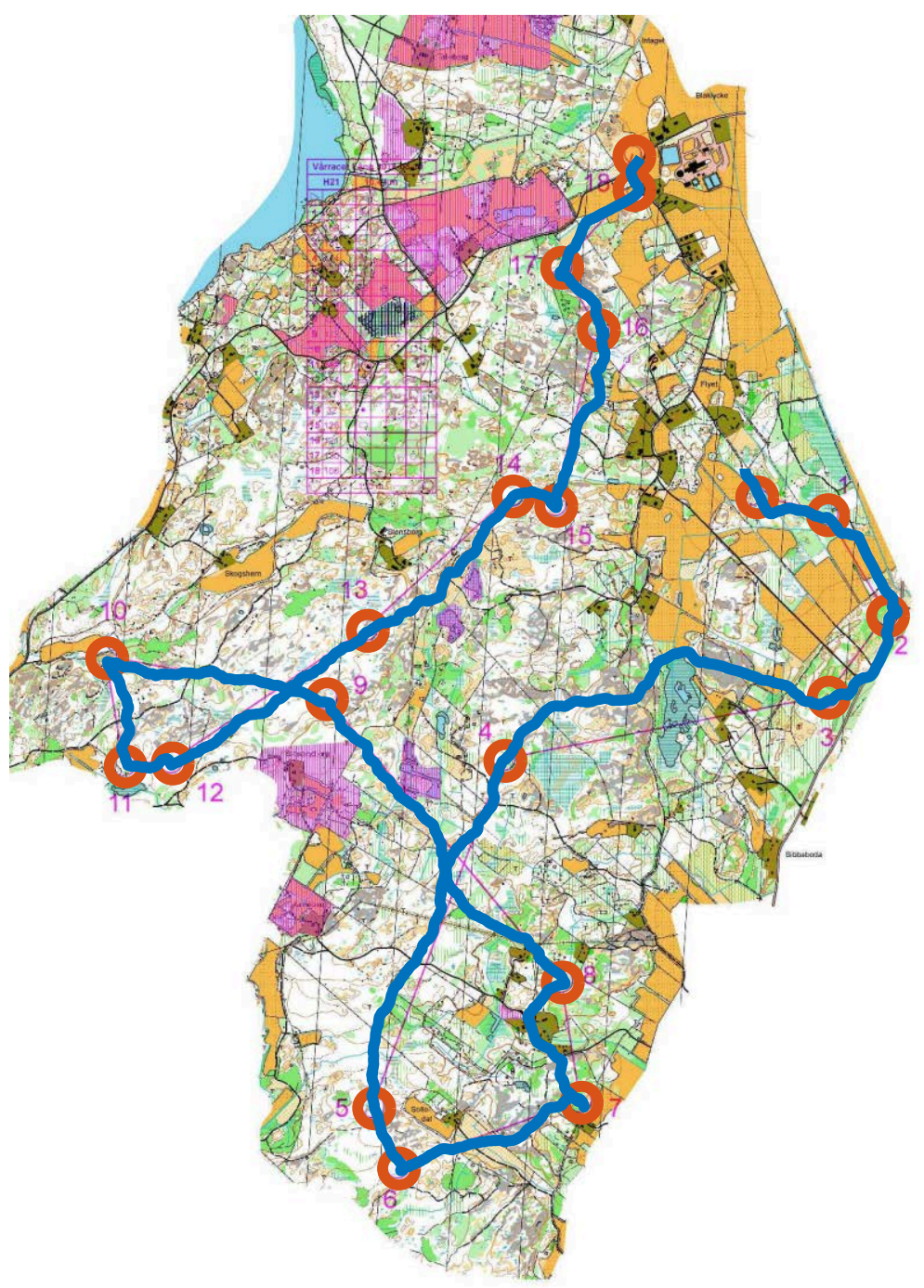

Figure 2.7: The position of a sprinter in an orienteering race as measured by a GNSS is shown in blue. The known location of the controls are shown in red. Figure courtesy of OK Orion. 
interferometer (TRI) [190]. In [142] an outline of a system for integrated adaptive sea ice tracking and multi-agent path planning is proposed. At the core of tracking of individual sea ice objects from various data sources lies the problem of multi-target multi-sensor tracking, including the problem of associating incoming measurements to existing targets or newly created ones.

Application 2.7 introduces a scenario where the aim is to track sea ice using radar in a fjord on Svalbard, Norway. The application is used to demonstrate the state-of-the-art target tracking framework that is improved in Contribution $\mathrm{V}$, as presented in Section 6.4.3. It is also used to evaluate the proposed method in Contribution I for learning the behaviour of targets while tracking, which is presented in Section 3.9. The proposed solution for the tracking application and related background theory were published by the author in [141, (C) 2017 IEEE]. This application was also considered in the publications [140] and [142] by the author. The derivations, results and analysis for the learning application has been submitted and is under review for publication in IEEE Transactions on Aerospace and Electronic Systems [184].

\section{Application 2.7: Tracking of Sea Ice}

Sea ice in Kongsfjorden on Svalbard is tracked in this application using a TRI. The dataset considered was gathered in a field campaign by the Norwegian Centre for Integrated Remote Sensing and Forecasting for Arctic Operations (CIRFA) in April 2016 using a Gamma portable radar interferometer. The dataset consists of radar samples taken every 3 min over a period of $41 \mathrm{~h}$. See Figure 2.8 for a single scan from this dataset transformed into Cartesian coordinates. The purpose is to predict the movement of drift ice in order to avoid collisions so that ships can traverse the region safely.

The application is a classic target tracking problem using a radar, with a large number of targets hidden in a sea of noise. One approach considered is to apply a recent development within multi-target tracking in the form of the LMB filter [154, 197].

A challenge for this problem is that the targets are not independent as they are submersed in the same currents and subject to the same wind. This correlation is ignored in the tracking application. A method for learning the dynamics of the ice to improve tracking performance is also considered in another application, however, data association is disregarded in this context.

The goal is to construct a complete target tracking solution from radar measurements to tracks of individual sea ice objects. To achieve this goal methods for image processing, target tracking using an LMB filter and modelling of dynamic systems are used. In addition, new methods for the complete solution are required for:

- storing information efficiently; and

- the implementation of the LMB filter.

The data is generously provided by Norut. The pre-processing of the data is presented in Applications 4.7 and 4.4 and the solutions to both applications are 


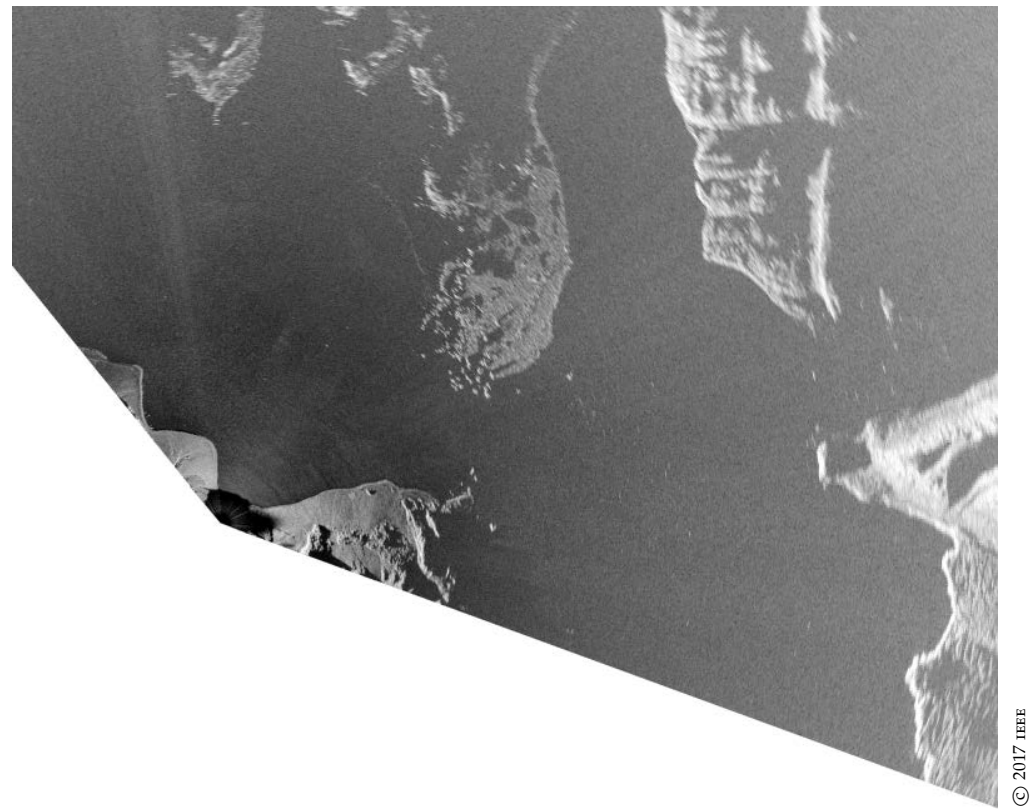

Figure 2.8: Example data from the TRI dataset. The image shows backscattered intensity, measured by a terrestrial radar. Figure courtesy of Norut. Published in [141].

presented throughout the thesis. All application descriptions with the word sea ice tracking in the caption are related to the tracking application. The model is described in Application 3.1 and the results are presented in Application 6.4. All application descriptions with the word sea ice learning in the caption are related to the learning application. The model is described in Application 3.11 and the results are presented in Application 5.11.

\subsection{Gaussian Process Motion Model Scenarios}

In target tracking it is common to use general purpose motion models, e.g. the near constant velocity model (NCVM), to predict the behaviour of targets. However, particular behaviours of the targets are often observed and it is desirable to use this information to improve predictions over time. The behaviour of all targets may be influenced by a common process, e.g., in the form of

- preferred paths in open terrain or indoors;

- obstacles that are avoided or common destinations;

- velocity or acceleration profiles in racing events;

- wind affecting flying vehicles; or 
- currents in the water affecting animals and ships.

For a single dynamical system an approximate model can often be determined beforehand, but it might be difficult to obtain an accurate model due to unknown influences from the environment or variations over time. For such systems estimation and prediction could be improved by using the available information to learn and gradually improve and adapt the model.

In target tracking it has long been standard practice to use interacting multiple models (IMM) [32] to consider various behaviours for targets. However, the behaviours are determined at design time and are not adapted to new data, so this approach is not suitable for the influences mentioned previously.

An approach to model a behaviour is described in [46], where the system pulls towards a given mean. The mean can be based on historic data and therefore work well to model preferred paths and velocity profiles. The method is shown in $[128,145]$ to improve predictions of ship trajectories. A similar ship prediction problem is solved using an NN approach in [89]. However, the methods grow in complexity with the size of the datasets, since new data is matched against all historic data, and it is not obvious from the methods how to appropriately compute the mean. Another approach to model particular behaviour is described in [82], where road maps are used to position cars. This approach is, however, limited to applications where the map is known in advance.

In computer vision, it is common to model motion patterns across the image. These motion patterns show a resemblance to the method proposed in this thesis. In $[59,105,174]$ trajectories in images are extracted and clustered, whereupon the estimated positions and velocities are used to train a GP dynamic model for each cluster. These are offline methods, where the motion pattern is only loosely coupled with the target motion model. An online adaptation is proposed in [59] by recomputing the model using only the latest data. Another approach is taken in [173] where the shapes of clustered trajectories instead are modelled as sparse GP.

This thesis proposes an approach for joint state estimation and online learning of influences in a dynamical system using recursive GP regression. The system function consists of a known linear term and an unknown nonlinear term of influence functions. The influence functions are modelled as independent GPS [151] with state-dependent input. Either they model the entire dynamics, in which case the linear term reduces to a prior mean function of the GPS, or they model a particular influence on the system that is difficult to determine at design time. The advantage of the latter is that the influence can have a lower dimension than the state vector. When tracking several targets many observations are available to estimate the shared influence, improving the performance, and additionally, it is possible to track slowly changing influences over time.

To estimate the influence functions, they are in turn modelled as sparse GPS using the fully independent conditional (FIC) approximation [148]. The function values are assumed to be independent conditioned on a number of inducing variables corresponding to the function values at a set of inducing inputs. The inducing inputs are selected prior to the estimation as a grid over the region of operation in the input space. With this model, each inducing variable reduces 
to a state augmented to the joint SSM of all dynamical systems. Inference in this model is then performed using an EKF [164], due to the nonlinearity caused by the dependence of the input to the function on the state.

The advantages of the proposed method compared to state-of-the-art are that

- inference and learning for dynamical systems are integrated into a single framework;

- the complexity does not increase with larger datasets;

- learning is performed recursively online for the whole or part of a system; and

- time-varying influences can be modelled.

Due to the large dimensionality of the augmented state space and the need to maintain cross-covariances, the computational complexity can be high. To mitigate this problem, and allow for real-time implementations, possible approximations are discussed.

System identification using GP priors on the transition and measurement functions are studied in, e.g., [52, 67, 176], using various approaches for estimating hyperparameters and latent variables. However, these methods do not directly extend to online learning, which is considered in this thesis.

Several sparse approximations of GPs have been proposed in the last decades, see [148]. The advantage of sparse approximations is that computational and memory complexities are reduced. A model that also allows for sequential estimation is proposed in [92] and a similar approach is presented in [28]. The latter is expanded into a full framework in [29] for online identification of ARX models, taking noisy inputs into consideration. The method in [92] was successfully used in [191] with direct measurements of inducing variables augmented to the states, as opposed to the indirect measurements considered here. A similar approach using direct measurements is also described in [108]. These methods together with the descriptions in [148] work as the basis for the approach to inference and learning in ssms proposed in this thesis.

For the sea ice learning application introduced in Application 2.7, the goal is to improve the prediction and the estimation of the position of sea ice by estimating the acceleration of the ice caused by the winds and currents. Data association is disregarded by using an association hypothesis obtained with a standard tracker. To achieve this goal the EKF is used for estimation. In addition, new methods are required for:

- modelling the dynamics of targets using GPs; and

- online learning of the dynamics using sparse approximations of GPS.

Application 2.8 introduces a scenario where multiple targets are tracked. The velocity of all targets is given as a function of the position. The main contribution presented with this scenario is a method to learn the behaviour of targets while being tracked. The derivations, results and analysis for the learning applications have been submitted for publication by the author. 


\section{Application 2.8: Velocity Field Scenario}

A simulated scenario of 200 targets is considered in this application. Noisy measurements of their positions are obtained with the correct association of each target. The velocities of the targets are given by a function of their positions, shown in Figure 2.9. This function is assumed to be unknown.

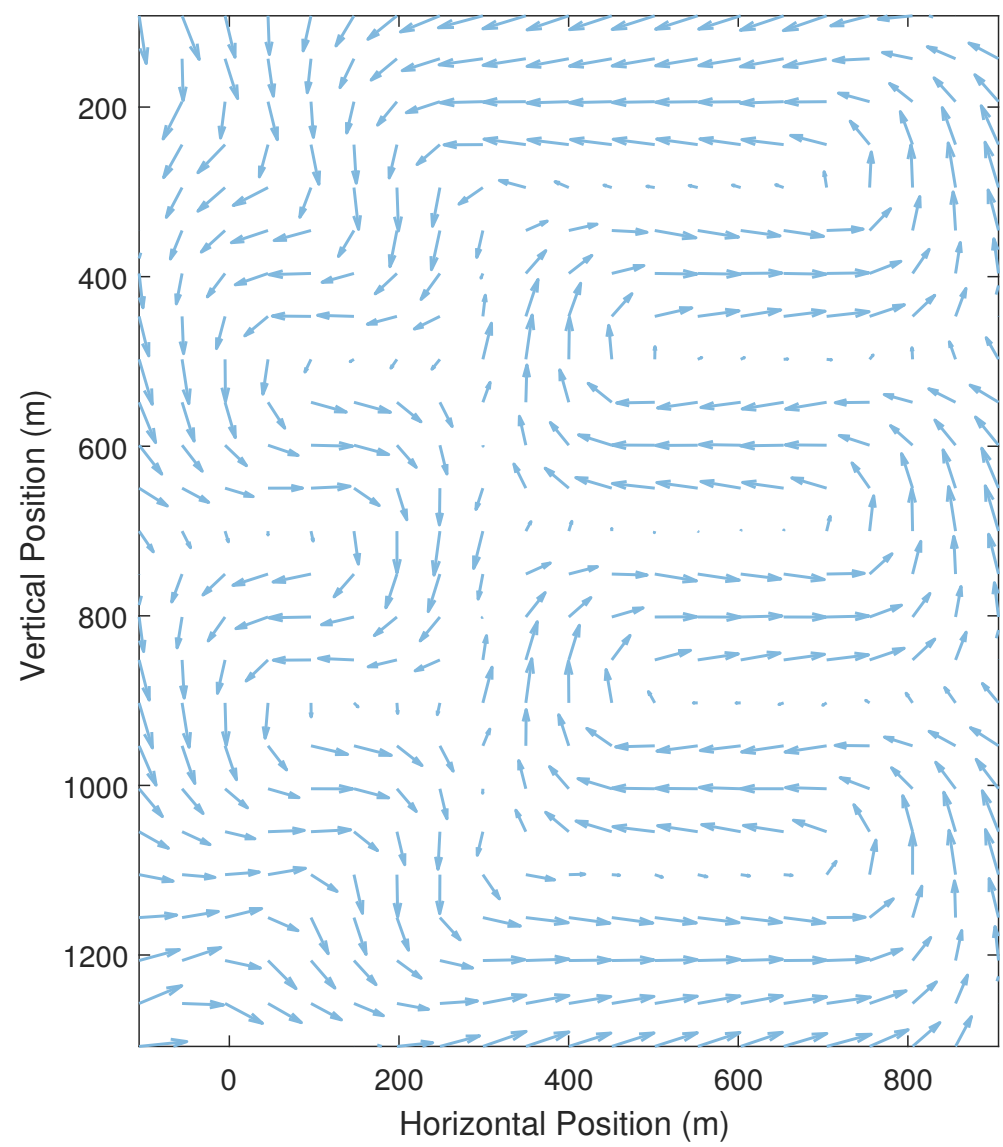

Figure 2.9: The velocity function common to all targets shown as a quiver plot.

The goal is to improve the prediction and the estimation of the position of targets by estimating the stationary velocity function common to all targets. To achieve this goal, the same methods as for the sea ice learning application are required. The solution is presented throughout the thesis, where all application descriptions with the word velocity field in the caption are related to this application. The model is presented in Application 3.10 and the posterior and estimators for the application are presented in Application 5.10. 



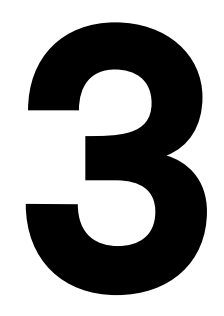

\section{State-Space Models}

A state-space model (SSM) is a mathematical model of a system where all necessary information required to predict the system behaviour is condensed into a state vector [6]. In essence, an SSM is a system of first-order differential or difference equations representing the dynamics of the system together with a measurement equation. Often the dynamics of the underlying system is continuous in time. However, observations in computerized systems are obtained in discrete time and the state can only be represented in a finite number of time steps. A common approach is therefore to initially model the dynamics in continuous time and then discretize the model to allow for inference.

Real systems are rarely deterministic, but exhibit random behaviour that cannot be predicted deterministically. This is modelled using a stochastic SSM, where stochastic is implied in the sequel, obtained by introducing stochastic variables into the ssm [6]. Although it is possible to model randomness in continuous time, stochastic variables will only be considered in discrete time in this thesis. A number of standard estimation approaches for the models introduced in this chapter are presented in Sections 5.1-5.3.

The outline of the chapter is as follows: A few structures and representations of the discrete-time and continuous-discrete ssms are introduced in Sections 3.1 and 3.2. Sensor models are outlined in Section 3.3 and a few common dynamic models are described in Section 3.4. In Section 3.5 a motion model for targets constrained to a region is derived. The model was first presented by the author in [181]. In Section 3.6 the JMM is described and an extension is proposed by the author. The extension to the model was first described by the author in [27]. A model for extended targets is described in Section 3.7. In Section 3.8 a variation of the SSM is presented for systems where observations have uncertain timestamps, which was partly presented by the author in [182]. A novel model is proposed in Section 3.9, where environmental influences are learned online. 


\subsection{Discrete-Time State-Space Models}

A discrete-time ssm can be represented in a number of ways and a few useful representations are presented in this section. Introductions to SSMs in a stochastic framework are given in, e.g., [6, 115].

The most general model description of a discrete-time system considered is the probabilistic state-space model (PSSM) [159], where the probability density functions (PDF) are modelled directly. The PSSM is defined as

$$
\begin{aligned}
\mathbf{x}_{0} & \sim p\left(\mathbf{x}_{0}\right), & & \\
\mathbf{x}_{k} \mid \mathbf{x}_{k-1} & \sim p\left(\mathbf{x}_{k} \mid \mathbf{x}_{k-1}\right), & & k \in \mathcal{K}=\{1: K\}, \\
\mathbf{y}_{j} \mid \mathbf{x}_{j} & \sim p\left(\mathbf{y}_{j} \mid \mathbf{x}_{j}\right), & & j \in \mathcal{J} \subseteq \mathcal{K},
\end{aligned}
$$

where

- $\mathbf{x}_{k} \in \mathbb{R}^{n}$ is the state vector at time $t_{k}$;

- $\mathbf{y}_{j} \in \mathbb{R}^{m}$ is an observation of the state vector at time $t_{j}$;

- $p\left(\mathbf{x}_{k} \mid \mathbf{x}_{k-1}\right)$ is the dynamic model of the system represented as a conditional PDF;

- $p\left(\mathbf{y}_{j} \mid \mathbf{x}_{j}\right)$ is the observation model of the system represented as a conditional PDF; and

- $p\left(\mathbf{x}_{0}\right)$ is the PDF for the initial state.

The dynamic and observation models are assumed to be Markovian in the sense that

$$
\begin{aligned}
p\left(\mathbf{x}_{k} \mid \mathcal{X}_{1: k-1}, \mathcal{Y}_{1: k-1}\right) & =p\left(\mathbf{x}_{k} \mid \mathbf{x}_{k-1}\right), \\
p\left(\mathbf{y}_{j} \mid \mathcal{X}_{1: j}, \mathcal{Y}_{1: j-1}\right) & =p\left(\mathbf{y}_{j} \mid \mathbf{x}_{j}\right)
\end{aligned}
$$

where $\mathcal{X}_{1: a}=\left\{\mathbf{x}_{k}\right\}_{k \in\{1: a\}}$ and $\mathcal{Y}_{1: a}=\left\{\mathbf{y}_{j}\right\}_{j \in\{1: a\} \cap \mathcal{J}}$ are the sets of all states and measurements, respectively, up to time $a$. When $\mathcal{J}=\mathcal{K}$ or when there is no ambiguity, index $k$ is used instead of $j$ for measurements. This model structure is convenient for deriving the posterior distribution, but often it is simpler to model a system on the slightly more restrictive structure of the nonlinear statespace model (NSSM) [6].

The NSSM is defined as the PSSM with the constraint that

$$
\begin{aligned}
& \mathbf{x}_{k}=\mathbf{f}_{k}\left(\mathbf{x}_{k-1}, \mathbf{w}_{k}\right), \\
& \mathbf{y}_{j}=\mathbf{h}_{j}\left(\mathbf{x}_{j}, \mathbf{v}_{j}\right),
\end{aligned}
$$

where transition function $\mathbf{f}_{k}(\mathbf{x}, \mathbf{w})$ and measurement function $\mathbf{h}_{j}(\mathbf{x}, \mathbf{v})$ are arbitrary nonlinear functions, often with the time index omitted, and

$$
\begin{aligned}
\mathbf{w}_{k} & \sim p\left(\mathbf{w}_{k}\right), \\
\mathbf{v}_{j} & \sim p\left(\mathbf{v}_{j}\right) .
\end{aligned}
$$


A common special case of the NSSM is when the dynamic model is a nonlinear function of the state and white Gaussian noise enters the model additively, resulting in the additive white Gaussian noise state-space model (AWGNSSM).

The AWGNSSM is defined as the NSSM with certain constraints. The additive noise constraint on the SSM is that

$$
\begin{aligned}
& \mathbf{x}_{k}=\mathbf{f}_{k}\left(\mathbf{x}_{k-1}\right)+\mathbf{w}_{k}, \\
& \mathbf{y}_{j}=\mathbf{h}_{j}\left(\mathbf{x}_{j}\right)+\mathbf{v}_{j} .
\end{aligned}
$$

The white Gaussian noise constraint on the noise distributions is that

$$
\begin{aligned}
\mathbf{w}_{k} & \sim \mathcal{N}\left(\mathbf{0}, \mathbf{Q}_{k}\right), \\
\mathbf{v}_{j} & \sim \mathcal{N}\left(\mathbf{0}, \mathbf{R}_{j}\right)
\end{aligned}
$$

and on the initial state that

$$
\mathbf{x}_{0} \sim \mathcal{N}\left(\overline{\mathbf{x}}_{0}, \mathbf{P}_{0}\right),
$$

where $\mathbf{Q}_{k} \in S_{+}^{n}, \mathbf{R}_{j} \in S_{+}^{m}, \mathbf{P}_{0} \in S_{++}^{n}$ and the PDF of the Gaussian distribution is given by

$$
\mathcal{N}(\mathbf{x} \mid \boldsymbol{\mu}, \boldsymbol{\Sigma})=|2 \pi \boldsymbol{\Sigma}|^{-\frac{1}{2}} \exp \left(-\frac{1}{2}(\mathbf{x}-\boldsymbol{\mu})^{\top} \boldsymbol{\Sigma}^{-1}(\mathbf{x}-\boldsymbol{\mu})\right)
$$

The most important special case is obtained by further assuming the functions $\mathbf{f}_{k}(\mathbf{x})$ and $\mathbf{h}_{j}(\mathbf{x})$ in the AWGNSSM to be linear, resulting in the linear Gaussian state-space model (LGSSM) [6]. The reason for its importance is that since the initial state is Gaussian, the posterior distributions of all subsequent states and predicted measurements are also Gaussian [81], which simplifies inference in the model considerably, as described in Section 5.2.1. The LGSSM is defined as the AWGNSSM with the constraint that

$$
\begin{aligned}
& \mathbf{x}_{k}=\mathbf{F}_{k} \mathbf{x}_{k-1}+\mathbf{w}_{k}, \\
& \mathbf{y}_{j}=\mathbf{H}_{j} \mathbf{x}_{j}+\mathbf{v}_{j},
\end{aligned}
$$

where the transition matrix $\mathbf{F}_{k}$ and the measurement matrix $\mathbf{H}_{j}$ are assumed to be independent of the state $\mathbf{x}_{k}$.

Generally the transition functions (3.3a), (3.5a) and (3.9a) are functions of the discretization time $T_{k}=t_{k}-t_{k-1}$, where, for most models considered, the discretization time is constant and denoted $T_{k}=T$.

\subsection{Continuous-Discrete State-Space Models}

A continuous-discrete SSM is a stochastic differential equation (SDE) in continuous time with stochastic observations in discrete time. Although representations of general SDEs are possible, the scope will be limited to the time-invariant linear Gaussian case. As will be seen, such sDEs have solutions in continuous time that can be computed analytically for an arbitrary finite set of timestamps. 
The dynamical model is given by the SDE [94]

$$
d \mathbf{x}(t)=\mathbf{A x}(t) d t+d \boldsymbol{\beta}(t),
$$

where the stochastic process $\mathbf{x}(t) \in \mathbb{R}^{n}$ is the state vector at time $t, \mathbf{A}$ is the system matrix, $\boldsymbol{\beta}(t)$ is a Brownian motion process with $\mathbf{E}\left[d \boldsymbol{\beta}(t) d \boldsymbol{\beta}^{\top}(t)\right]=\mathbf{Q} d t$ and $\mathbf{Q}$ is a spectral density matrix. This is a special case of an Itô SDE and the integrals of the noise for non-overlapping intervals are assumed to be independent, so the process in (3.10) satisfies the Markov property. An independent prior on the state at time $t_{0}$ is given by $\mathbf{x}\left(t_{0}\right) \sim \mathcal{N}\left(\overline{\mathbf{x}}_{0}, \mathbf{P}_{0}\right)$. The measurement model, with independent noise, is

$$
\mathbf{y}_{j}=\mathbf{H}_{j} \mathbf{x}\left(t_{j}^{y}\right)+\mathbf{v}_{j}, \quad j \in \overline{\mathcal{J}},
$$

where $\mathbf{y}_{j} \in \mathbb{R}^{m_{y}}$ is a noisy measurement at time $t_{j}^{y}, \overline{\mathcal{J}}=\{1: J\}, \mathbf{H}_{j}$ is the observation matrix, $\mathbf{v}_{j} \sim \mathcal{N}\left(\mathbf{0}, \mathbf{R}_{j}\right)$ and the notations $\mathcal{Y}=\left\{\mathbf{y}_{j}\right\}_{j \in \overline{\mathcal{J}}}$ and $\mathcal{T}_{y}=$ $\left\{t_{j}^{y}\right\}_{j \in \overline{\mathcal{J}}}$ will be used. The ssm given by (3.10) and (3.11) is a Gauss-Markov process [94].

Remark 3.1: In discrete time it is often convenient if the measurement index $j \in \mathcal{J} \subseteq \mathcal{K}$ corresponds to the time index of the observed state. However, in continuous time, the set of time indices might not be available, in which case $j \in \overline{\mathcal{J}}=\{1: J\}$ and the corresponding time is given by $t_{j}^{y}$ in continuous time and $\bar{j}$ in discrete time.

The differential equation in (3.10) can be solved analytically for a given instant of time by converting the model to a discrete-time equivalent model [15, $68,192]$. The differential equation is discretized at time instants $\mathcal{T}_{p}$ of interest as well as time instants for which there are measurements or a prior, given by the set $\mathcal{T}_{x}=\mathcal{T}_{p} \cup \mathcal{T}_{y} \cup t_{0}=\left\{t_{k}\right\}_{k \in 0 \cup \mathcal{K}}$, where $\mathcal{K}=\{1: K\}$ and $t_{k-1}<t_{k}$. The discretized states are denoted $\mathcal{X}=\left\{\mathbf{x}_{k}\right\}_{k \in 0 \cup \mathcal{K}}$. The continuous-time state trajectory can be marginalized to only consider the states $\mathcal{X}_{p}=\{\mathbf{x}(t)\}_{t \in \mathcal{T}_{p}}$ at the instants of interest $\mathcal{T}_{p}$.

The equivalent discrete-time stochastic difference equation is obtained by integrating (3.10) over the time interval $\left[t_{k-1}, t_{k}\right]$

$$
\mathbf{x}\left(t_{k}\right)=e^{\mathbf{A}\left(t_{k}-t_{k-1}\right)} \mathbf{x}\left(t_{k-1}\right)+\int_{t_{k-1}}^{t_{k}} e^{\mathbf{A}\left(t_{k}-\tau\right)} d \boldsymbol{\beta}(\tau),
$$

resulting in the discrete-time equivalent model

$$
\begin{array}{rlrl}
\mathbf{x}_{k} & =\mathbf{F}_{k, k-1} \mathbf{x}_{k-1}+\mathbf{w}_{k, k-1}, & & k \in \mathcal{K}, \\
\mathbf{y}_{j} & =\mathbf{H}_{j} \mathbf{x}_{j}+\mathbf{v}_{j}, & & j \in \overline{\mathcal{J}}, \\
\mathbf{x}_{0} & \sim \mathcal{N}\left(\overline{\mathbf{x}}_{0}, \mathbf{P}_{0}\right), &
\end{array}
$$

where $\mathbf{x}_{k}=\mathbf{x}\left(t_{k}\right), \mathbf{w}_{k, k-1} \sim \mathcal{N}\left(\mathbf{0}, \mathbf{Q}_{k, k-1}\right), \bar{j} \in \mathcal{K}$ is defined such that $t_{\bar{j}}=t_{j}^{y}$,

$$
\mathbf{F}_{i, j}=e^{\mathbf{A}\left(t_{i}-t_{j}\right)},
$$




$$
\mathbf{Q}_{i, j}=\int_{0}^{t_{i}-t_{j}} e^{\mathbf{A} \tau} \mathbf{Q} e^{\mathbf{A}^{\top} \tau} d \tau,
$$

and full rank is assumed for $\mathbf{Q}_{i, j}$. Methods to compute the discretizations are described in [192]. Discretizations between $t_{k-1}$ and $t_{k}$ will be denoted by the subscript $k$ for convenience. The matrices in (3.14) satisfy [192]

$$
\begin{aligned}
\mathbf{F}_{k, k-1} & =\mathbf{F}_{k, \tau} \mathbf{F}_{\tau, k-1}, \\
\mathbf{Q}_{k, k-1} & =\mathbf{F}_{k, \tau} \mathbf{Q}_{\tau, k-1} \mathbf{F}_{k, \tau}^{\top}+\mathbf{Q}_{k, \tau},
\end{aligned}
$$

for all $t_{\tau} \in\left[t_{k-1}, t_{k}\right]$.

\subsection{Sensor Models}

To enable inference in a motion model, observations of the state vectors are required. The sensor model describes the relationship between the state vector and the stochastic observation. A sensor model can be described by a conditional PDF, as in (3.1c). However, it is often difficult to accurately identify the PDF of the sensor.

A convenient approximation is obtained by modelling the deterministic function $\mathbf{h}(\mathbf{x})$ of the state independently from the noise. The noise $\mathbf{v}_{j} \sim p\left(\mathbf{v}_{j}\right)$ in the sensor can then be modelled to enter additively as

$$
\mathbf{y}_{j}=\mathbf{h}\left(\mathbf{x}_{j}\right)+\mathbf{v}_{j}, \quad j \in \mathcal{J} .
$$

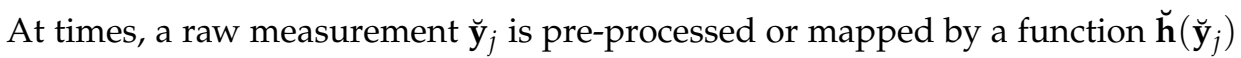
prior to considering noise. This results in

$$
\breve{\mathbf{h}}\left(\breve{\mathbf{y}}_{j}\right)=\mathbf{y}_{j}=\mathbf{h}\left(\mathbf{x}_{j}\right)+\mathbf{v}_{j}
$$

where the noise is modelled to enter after the pre-processing and may simplify the estimation.

If the measurement noise distribution is difficult to characterize, it is often convenient to approximate it with a zero-mean Gaussian with a reasonable covariance matrix $\mathbf{R}_{j}$. The matrix is chosen to compensate for measurement noise as well as model errors, and should rather be seen as the uncertainty of the measurement.

The function $\mathbf{h}(\mathbf{x})$, or at least its structure, can often be determined from the physical characteristics or geometry of the sensor. For many sensors it is common that the measurement pre-processing results in a direct observation of the position of the target, which is modelled as the linear function $\mathbf{h}(\mathbf{x})=$ $(x, y)^{\top}$. For other sensors the detection might be the range or the angle to the target. The range is modelled as $\mathbf{h}(\mathbf{x})=\sqrt{x^{2}+y^{2}}$ and the angle is modelled as $\mathbf{h}(\mathbf{x})=\arctan 2(y, x)$, which are nonlinear functions of the state. More advanced models for camera applications are described in Chapter 4. 


\subsection{Motion Models}

In target tracking applications, the state vector commonly represents the position and motion of the target being tracked, so $\mathbf{f}_{k}(\mathbf{x}, \mathbf{w})$ in (3.3a) is therefore referred to as a motion model. The model can be tailored to a particular type of target or application, or a suitable general motion model can be tuned to fit the application. See [114] for a selection of common motion models.

It is often difficult to model the target accurately. If the target is intelligent, e.g., if the target is a human, an animal or a vehicle controlled by a human, the intelligent part of the behaviour must often be modelled as noise. Occasionally one can make assumptions on constraints on the motion of the expected target type, e.g., a fixed wing aircraft has limited turning radius and cars generally stay on roads, which can be encoded into the model.

First, the dimension $p$ of the position of the target is determined. The dimension $p$ is normally 1, 2 or 3 depending on the mobility of the target. For motion in a plane, such as on the ground, a two-dimensional model is often sufficient, while for aerial targets motion in all three dimensions is possible. The first states of the state vector in a motion model are chosen as the $p$-dimensional position.

The simplest model is to assume that the position is nearly constant, with a zero-mean stochastic velocity, commonly referred to as a random walk model, constant position model or nearly constant position model (NCPM) [114]. The state vector is modelled by the position, which for two dimensions and omitted time index is given by

$$
\mathbf{x}=\left(\begin{array}{ll}
x & y
\end{array}\right)^{\top}
$$

The NCPM is modelled as (3.10) in continuous time with $\mathbf{A}=0 \otimes \mathbf{I}_{p}$ and $\mathbf{Q}=$ $\sigma^{2} \otimes \mathbf{I}_{p}$, resulting in a discretized linear Gaussian motion model on the form (3.9a) with

$$
\mathbf{F}_{k}=\mathbf{I}_{p} \quad \text { and } \quad \mathbf{Q}_{k}=\sigma^{2} T_{k} \otimes \mathbf{I}_{p}
$$

where $\otimes$ is the Kronecker product. Initialization of the state is trivially chosen as Gaussian with the mean and covariance of the first measurement.

A slightly more complex, but often useful, model is obtained by assuming that the velocity is nearly constant, commonly referred to as a constant velocity model or a nearly constant velocity model (NCVM) [114]. The NCVM can be seen as an extension of the NCPM where the state vector has been augmented by the velocity, which for two dimensions and omitted time index becomes

$$
\mathbf{x}=\left(\begin{array}{llll}
x & y & \dot{x} & \dot{y}
\end{array}\right)^{\top} .
$$

The NCVM is modelled as (3.10) in continuous time with

$$
\mathbf{A}=\left(\begin{array}{ll}
0 & 1 \\
0 & 0
\end{array}\right) \otimes \mathbf{I}_{p} \quad \text { and } \quad \mathbf{Q}=\left(\begin{array}{cc}
0 & 0 \\
0 & \sigma^{2}
\end{array}\right) \otimes \mathbf{I}_{p}
$$


resulting in a discretized linear Gaussian motion model with

$$
\mathbf{F}_{k}=\left(\begin{array}{cc}
1 & T_{k} \\
0 & 1
\end{array}\right) \otimes \mathbf{I}_{p} \quad \text { and } \quad \mathbf{Q}_{k}=\sigma^{2}\left(\begin{array}{cc}
\frac{T_{k}^{3}}{3} & \frac{T_{k}^{2}}{2} \\
\frac{T_{k}^{2}}{2} & T_{k}
\end{array}\right) \otimes \mathbf{I}_{p}
$$

Initialization of the state is trivially chosen as Gaussian with the mean and covariance of the first measurement for the position and mean zero and covariance $v_{\max }^{2} / 3$ for the velocity, where $v_{\max }$ is a bound on the velocity of the target [122]. The scaling of the covariance is due to an assumption of a uniform distribution of the velocity in the range $\left[-v_{\max }, v_{\max }\right]$.

Continuing in the same fashion, the nearly constant acceleration model (NCAM) can be derived in discrete time as a linear Gaussian motion model with

$$
\mathbf{F}_{k}=\left(\begin{array}{ccc}
1 & T_{k} & \frac{T_{k}^{2}}{2} \\
0 & 1 & T_{k} \\
0 & 0 & 1
\end{array}\right) \otimes \mathbf{I}_{p} \quad \text { and } \quad \mathbf{Q}_{k}=\sigma^{2}\left(\begin{array}{ccc}
\frac{T_{k}^{5}}{20} & \frac{T_{k}^{4}}{8} & \frac{T_{k}^{3}}{6} \\
\frac{T_{k}^{4}}{8} & \frac{T_{k}^{3}}{3} & \frac{T_{k}^{2}}{2} \\
\frac{T_{k}^{3}}{6} & \frac{T_{k}^{2}}{2} & T_{k}
\end{array}\right) \otimes \mathbf{I}_{p}
$$

Initialization of the state is performed in the same fashion as for the NCVM with an additional bound on the acceleration of the target.

Another conventional motion model is the coordinated turn model (стм) [114], where the speed and turning rate are assumed to be nearly constant. The стм can be seen as an extension of the NCVM where the state vector has been augmented by the turning rate, which for two dimensions and omitted time index becomes

$$
\mathbf{x}=\left(\begin{array}{lllll}
x & y & \dot{x} & \dot{y} & \omega
\end{array}\right)^{\top} .
$$

The Стм is not linear, however, but is an additive white Gaussian noise motion model on the form (3.5a) with

$$
\mathbf{f}_{k}\left(\mathbf{x}_{k}\right)=\left(\begin{array}{c}
x_{k}+\frac{\dot{x}_{k}}{\omega_{k}} \sin \left(\omega_{k} T_{k}\right)-\frac{\dot{y}_{k}}{\omega_{k}}\left(1-\cos \left(\omega_{k} T_{k}\right)\right) \\
y_{k}+\frac{\dot{x}_{k}}{\omega_{k}}\left(1-\cos \left(\omega_{k} T_{k}\right)\right)+\frac{\dot{y}_{k}}{\omega_{k}} \sin \left(\omega_{k} T_{k}\right) \\
\dot{x}_{k} \cos \left(\omega_{k} T_{k}\right)-\dot{y}_{k} \sin \left(\omega_{k} T_{k}\right) \\
\dot{x}_{k} \sin \left(\omega_{k} T_{k}\right)+\dot{y}_{k} \cos \left(\omega_{k} T_{k}\right) \\
\omega_{k}
\end{array}\right),
$$

where the process noise for the last state is independent from the process noise of the other states.

The NCVM presented in this section is used in the sea ice tracking application as described in Application 3.1. 


\section{Application 3.1: Sea Ice Tracking Model}

The drift ice is modelled as an NCVM in two dimensions with a sampling time $T$ of $180 \mathrm{~s}$. The covariance parameter $\sigma^{2}$ is chosen as $1.7 \times 10^{-5} \mathrm{~m}^{2} / \mathrm{s}^{3}$ and the maximum velocity is chosen at $17.3 \mathrm{~m} / \mathrm{h}$. Despite clearly being correlated, the ice objects are, for now, assumed to be independent of each other.

The sea ice is observed using a radar, which delivers range-bearing measurements transformed into Cartesian coordinates. The measurement noise is modelled to be Gaussian in the transformed coordinates, resulting in the sensor model

$$
\mathbf{y}_{j}=\mathbf{H} \mathbf{x}_{j}+\mathbf{v}_{j}=\left(\begin{array}{llll}
1 & 0 & 0 & 0 \\
0 & 1 & 0 & 0
\end{array}\right) \mathbf{x}_{j}+\mathbf{v}_{j}
$$

where only the position of the ice object is observed and $\mathbf{v}_{j} \sim \mathcal{N}(\mathbf{0}, \mathbf{R})$. The measurement noise standard deviation is chosen as $12.2 \mathrm{~m}$ in each dimension. The position sensor model together with the motion model result in an LGSSM. The method of extracting measurements from the scans is described in Applications 4.7 and 4.4 .

\subsection{Constrained Motion Model}

Conventional motion models describe the relationship between the states over time, but do not include constraints on the values of the states. These models work well for many targets that can move freely. If the target is constrained to a particular region, it is possible to explicitly define constraints, but estimation for the model would result in a constrained optimization problem. An alternative approach is to design a model where predictions of the target remain inside the constrained region. Contribution III is one such model, denoted as a constrained motion model (CMM), which is developed by the author in [181] and also presented in this section. The estimation method considered for this model is a standard EKF, presented in Section 5.2.2.

\subsubsection{Motivation}

When a target is constrained to a region, adapting the motion model to reflect this can improve the tracking performance. In the following, this is achieved by making a few assumptions, listed in Assumption 3.1, about the target behaviour close to the boundary of the region. The inspiration comes from research on obstacle avoidance using potential fields [104].

Assumption 3.1: The assumptions made about the target motion are the following:

1. The target moves in two dimensions.

2. The target speed is nearly constant. 
3. The target avoids moving into the boundary by turning when it gets close.

4. The target aligns its velocity with the tangent of the boundary by turning when close to the boundary.

5. The target moves either in a clockwise or counter-clockwise direction along the boundary of the region, resulting in a preferred turning direction when avoiding collision and aligning with the boundary.

6. The strength of the influence from each point along the boundary is assumed to be a function of the state of the target and the point.

The state of the target is modelled with position $\mathbf{p}$ and velocity $\dot{\mathbf{p}}$ in two dimensions as

$$
\mathbf{x}=\left(\begin{array}{c}
\mathbf{p} \\
\dot{\mathbf{p}}
\end{array}\right)=\left(\begin{array}{c}
x \\
y \\
\dot{x} \\
\dot{y}
\end{array}\right)
$$

where the time index has been omitted. The angular velocity $\omega(\mathbf{x})$ of the target is modelled as the integrated effect on the target of each point along the boundary according to

$$
\omega(\mathbf{x})=d_{r}(\mathbf{x}) \int_{\mathbf{N}}\left(\beta_{d}+\beta_{a}\left(\dot{\mathbf{p}}_{\perp} \cdot \mathbf{l}(\mathbf{n})\right)\right) w(\mathbf{x}, \mathbf{n}) d \mathbf{n},
$$

where

- $\mathbf{N}$ denotes the set of all points along the boundary of the region;

- $d_{r}(\mathbf{x}) \in\{-1,1\}$ is the preferred rotational direction of the target;

- $\beta_{d}$ is a design parameter for the importance of avoiding collision with the boundary;

- $\beta_{a}$ is a design parameter for the importance of aligning with the boundary;

- $\mathbf{l}(\mathbf{n})$ is the tangent of the boundary in the point $\mathbf{n}$; and

- $w(\mathbf{x}, \mathbf{n})$ is the strength of the influence of a point $\mathbf{n}$ on a target with state $\mathbf{x}$.

The notation $(a, b)_{\perp}^{\top}=(b,-a)^{\top}$ is used, which rotates a two-dimensional vector $\pi / 2 \mathrm{rad}$ clockwise, and the operator $\mathbf{a} \cdot \mathbf{b}=\mathbf{a}^{\top} \mathbf{b}$ is the scalar product of two vectors. 


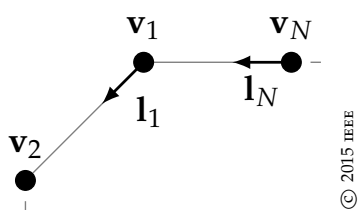

Figure 3.1: Polygon representation. Published in [181].

\subsubsection{Region Model}

The boundary of the constraint region is modelled as a simple two-dimensional polygon. The polygon is defined by $N$ vertices $\mathbf{v}_{i} \in \mathbb{R}^{2}, i \in\{1: N\}$, given in counter-clockwise order. Points on each segment of the polygon are obtained from

$$
\mathbf{n}_{i}(s)=\mathbf{v}_{i}+s \mathbf{l}_{i}, \quad s \in\left[0, m_{i}\right],
$$

where $m_{i}=\left\|\mathbf{v}_{i+1}-\mathbf{v}_{i}\right\|$ and $\mathbf{l}_{i}=\left(\mathbf{v}_{i+1}-\mathbf{v}_{i}\right) / m_{i}$, as shown in Figure 3.1, with obvious adjustments for $m_{N}$ and $\mathbf{l}_{N}$.

The strength of the influence $w(\mathbf{x}, \mathbf{n})$ in (3.28) for a point $\mathbf{n}_{i}(s)$ on the boundary is modelled to diminish as

$$
w\left(\mathbf{x}, \mathbf{n}_{i}(s)\right)=\frac{1}{\left\|\mathbf{p}-\mathbf{n}_{i}(s)\right\|^{2}}=\frac{1}{\left\|\mathbf{e}_{i}-s \mathbf{l}_{i}\right\|^{2}},
$$

where $\mathbf{e}_{i}=\mathbf{p}-\mathbf{v}_{i}$. Inserting (3.30) into (3.28) and using the region model in (3.29) gives the angular velocity

$$
\omega(\mathbf{x})=d_{r}(\mathbf{x}) \sum_{i=1}^{N}\left(\beta_{d}+\beta_{a}\left(\dot{\mathbf{p}}_{\perp} \cdot \mathbf{1}_{i}\right)\right) w_{i}(\mathbf{x}),
$$

where, using $\left\|\mathbf{1}_{i}\right\|=1$ and Proposition 3.1,

$$
\begin{aligned}
w_{i}(\mathbf{x}) & =\int_{0}^{m_{i}} w\left(\mathbf{x}, \mathbf{n}_{i}(s)\right) d s=\int_{0}^{m_{i}} \frac{1}{\left\|\mathbf{e}_{i}-s \mathbf{l}_{i}\right\|^{2}} d s \\
& =\frac{1}{\mathbf{e}_{i}^{\top} \mathbf{l}_{i \perp}} \arctan 2\left(m_{i} \mathbf{e}_{i}^{\top} \mathbf{1}_{i \perp},\left\|\mathbf{e}_{i}\right\|^{2}-m_{i} \mathbf{e}_{i}^{\top} \mathbf{l}_{i}\right) .
\end{aligned}
$$

The derivation of this relationship has been corrected compared to [180] which did not consider all special cases. Figure 3.2 illustrates (3.31) with $\beta_{a}=0$ for a constraint region.

Proposition 3.1: Given $\mathbf{a} \in \mathbb{R}^{2}, \mathbf{b} \in \mathbb{R}^{2}, s \in \mathbb{R}, c \in \mathbb{R}^{+}, \mathbf{a} \neq s \mathbf{b}, \forall s \in[0, c]$ and $\mathbf{b}^{\top} \mathbf{b}=1$, it holds that

$$
\int_{0}^{c} \frac{1}{\|\mathbf{a}-s \mathbf{b}\|^{2}} d s=\frac{1}{\mathbf{a}^{\top} \mathbf{b}_{\perp}} \arctan 2\left(c \mathbf{a}^{\top} \mathbf{b}_{\perp},\|\mathbf{a}\|^{2}-c \mathbf{a}^{\top} \mathbf{b}\right)
$$




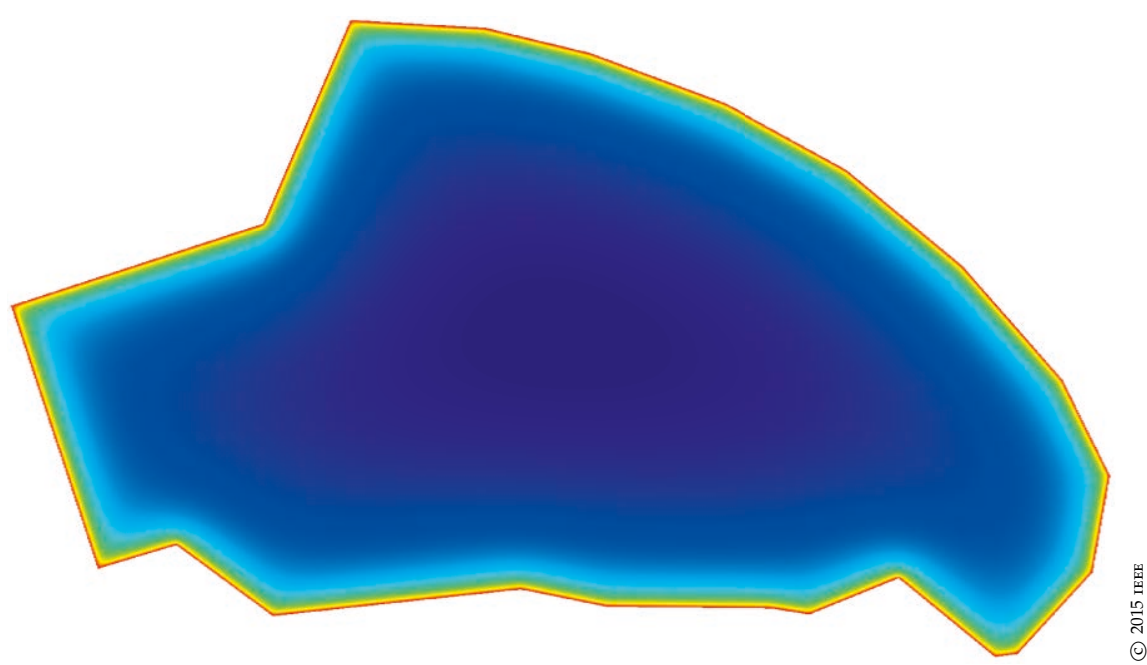

Figure 3.2: Potential field illustration. Published in [181].

Proof: The proof is presented in Appendix A.1.

The Jacobians of the weights are given by

$$
\frac{\partial w_{i}}{\partial \mathbf{x}}(\mathbf{x})=\left(\begin{array}{llll}
\frac{\partial w_{i}}{\partial x}(\mathbf{x}) & \frac{\partial w_{i}}{\partial y}(\mathbf{x}) & 0 & 0
\end{array}\right),
$$

where, using the notation $\mathbf{a}=\left(a_{x}, a_{y}\right)^{\top}$,

$$
\begin{aligned}
\frac{\partial w_{i}}{\partial x}(\mathbf{x}) & =\frac{1}{\mathbf{1}_{i}^{\top} \mathbf{e}_{i \perp}}\left(l_{i y} w_{i}(\mathbf{x})+\frac{e_{i y}}{\left\|\mathbf{e}_{i}\right\|^{2}}-\frac{e_{i y}-l_{i y} m_{i}}{\left\|\mathbf{e}_{i}\right\|^{2}-2 \mathbf{1}_{i}^{\top} \mathbf{e}_{i} m_{i}+m_{i}^{2}}\right), \\
\frac{\partial w_{i}}{\partial y}(\mathbf{x}) & =\frac{1}{\mathbf{1}_{i}^{\top} \mathbf{e}_{i \perp}}\left(-l_{i x} w_{i}(\mathbf{x})-\frac{\boldsymbol{e}_{i x}}{\left\|\mathbf{a}_{i}\right\|^{2}}+\frac{\boldsymbol{e}_{i x}-l_{i x} m_{i}}{\left\|\mathbf{e}_{i}\right\|^{2}-2 \mathbf{l}_{i}^{\top} \mathbf{e}_{i} m_{i}+m_{i}^{2}}\right) .
\end{aligned}
$$

The direction of the rotation $d_{r}(\mathbf{x})$ can either be chosen using prior information or be estimated by comparing the target velocity $\dot{\mathbf{p}}$ to the boundary directions $\mathbf{l}_{i}$, e.g. using

$$
d_{r}(\mathbf{x})=\operatorname{sign}\left(\sum_{i=1}^{N}\left(\dot{\mathbf{p}} \cdot \mathbf{1}_{i}\right) w_{i}(\mathbf{x})\right) .
$$

Another option is to consider two models, one for each direction.

\subsubsection{Motion Model}

The motion model chosen is a CTM with known angular velocity [114]. The state vector is given by (3.27) and the deterministic motion model in continuous time is

$$
\dot{\mathbf{x}}(t)=\mathbf{F}_{c}(\omega(\mathbf{x}(t))) \mathbf{x}(t),
$$


where

$$
\mathbf{F}_{c}(\omega)=\left(\begin{array}{cccc}
0 & 0 & 1 & 0 \\
0 & 0 & 0 & 1 \\
0 & 0 & 0 & -\omega \\
0 & 0 & \omega & 0
\end{array}\right)
$$

With a zero-order hold assumption on $\omega(\mathbf{x}(t))=\omega$ in (3.37) and a discretization time of $T,(3.38)$ is discretized [157] exactly as

$$
\mathbf{F}(\omega)=\exp \left(\mathbf{F}_{c}(\omega) T\right)=\left(\begin{array}{cccc}
1 & 0 & \frac{\sin \omega T}{\omega} & -\frac{1-\cos \omega T}{\omega} \\
0 & 1 & \frac{1-\cos \omega T}{\omega} & \frac{\sin \omega T}{\omega} \\
0 & 0 & \cos \omega T & -\sin \omega T \\
0 & 0 & \sin \omega T & \cos \omega T
\end{array}\right) .
$$

Introducing additive white Gaussian noise results in the discrete-time motion model

$$
\mathbf{x}_{k}=\mathbf{f}\left(\mathbf{x}_{k-1}, \omega\left(\mathbf{x}_{k-1}\right)\right)+\mathbf{w}_{k}=\mathbf{F}\left(\omega\left(\mathbf{x}_{k-1}\right)\right) \mathbf{x}_{k-1}+\mathbf{w}_{k},
$$

where $\mathbf{f}(\mathbf{x}, \omega)=\mathbf{F}(\omega) \mathbf{x}, \mathbf{w}_{k} \sim \mathcal{N}\left(\mathbf{0}, \mathbf{Q}_{k}\right)$ and $\mathbf{Q}_{k}$ is defined as in (3.22). Reintroducing $\omega=\omega(\mathbf{x})$, the Jacobian with regards to $\mathbf{x}$ is

$$
\frac{d \mathbf{f}}{d \mathbf{x}}(\mathbf{x}, \omega(\mathbf{x}))=\frac{\partial \mathbf{f}}{\partial \mathbf{x}}(\mathbf{x}, \omega(\mathbf{x}))+\frac{\partial \mathbf{f}}{\partial \omega}(\mathbf{x}, \omega(\mathbf{x})) \frac{\partial \omega(\mathbf{x})}{\partial \mathbf{x}},
$$

using the chain rule, where, using (3.35),

$$
\begin{gathered}
\frac{\partial \mathbf{f}}{\partial \mathbf{x}}(\mathbf{x}, \omega)=\left(\begin{array}{cccc}
1 & 0 & \frac{\sin \omega T}{\omega} & -\frac{1-\cos \omega T}{\omega} \\
0 & 1 & \frac{1-\cos \omega T}{\omega} & \frac{\sin \omega T}{\omega} \\
0 & 0 & \cos \omega T & -\sin \omega T \\
0 & 0 & \sin \omega T & \cos \omega T
\end{array}\right), \\
\frac{\partial \mathbf{f}}{\partial \omega}(\mathbf{x}, \omega)=\left(\begin{array}{c}
\frac{(\omega T \dot{x}-\dot{y}) \cos (\omega T)-(\dot{x}+\omega T \dot{y}) \sin (\omega T)+\dot{y}}{\omega^{2}} \\
\frac{(\dot{x}-\omega T \dot{y}) \cos (\omega T)+(\dot{y}-\omega T \dot{x}) \sin (\omega T)-\dot{x}}{\omega^{2}} \\
-T \dot{y} \cos (\omega T)-T \dot{x} \sin (\omega T) \\
T \dot{x} \cos (\omega T)-T \dot{y} \sin (\omega T)
\end{array}\right), \\
\frac{\partial \omega}{\partial \mathbf{x}}(\mathbf{x})=d_{r}(\mathbf{x}) \sum_{i=1}^{N}\left(\begin{array}{c}
\left(\beta_{d}+\beta_{a} \dot{\mathbf{p}}_{\perp}^{\top} \mathbf{l}_{i}\right) \frac{\partial w^{i}}{\partial x}(\mathbf{x}) \\
\left(\beta_{d}+\beta_{a} \dot{\mathbf{p}}_{\perp}^{\top} \mathbf{l}_{i}\right) \frac{\partial w^{i}}{\partial y}(\mathbf{x}) \\
-\beta_{a} l_{i y} w^{i}(\mathbf{x}) \\
\beta_{a} l_{i x} w^{i}(\mathbf{x})
\end{array}\right) .
\end{gathered}
$$

Care is needed in implementation as $\omega \rightarrow 0$ for $(3.42 a)$ and $(3.42 b)$, which reduce to

$$
\lim _{\omega \rightarrow 0} \frac{\partial \mathbf{f}}{\partial \mathbf{x}}(\mathbf{x}, \omega)=\left(\begin{array}{cccc}
1 & 0 & T & 0 \\
0 & 1 & 0 & T \\
0 & 0 & 1 & 0 \\
0 & 0 & 0 & 1
\end{array}\right)
$$




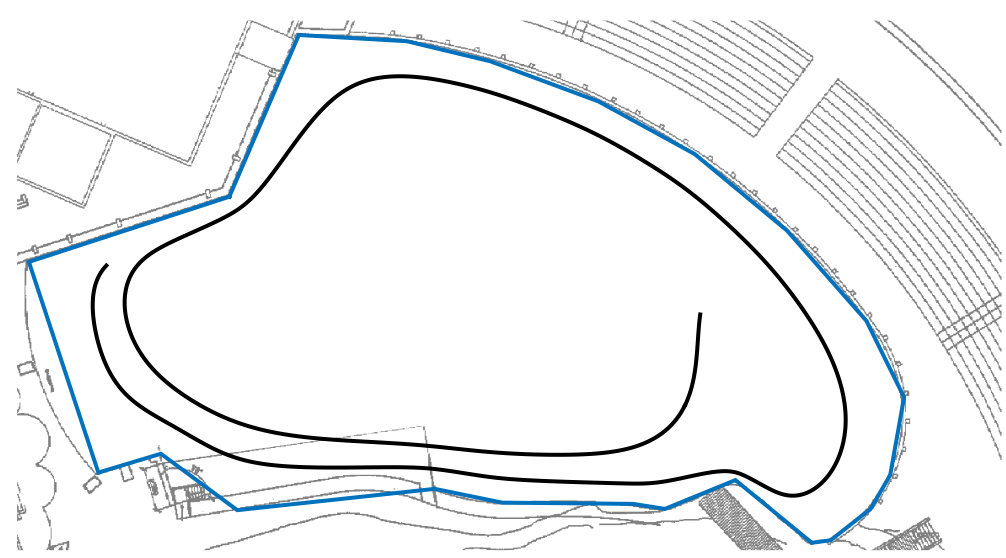

Figure 3.3: The basin edges are approximately described as a polygon, shown in blue. Straight segments of the edge have natural vertices for the polygon while curved segments require frequent vertices to achieve a reasonable approximation. Inside the basin is a simulation of a dolphin swimming according to the CMM in a clockwise manner.

$$
\lim _{\omega \rightarrow 0} \frac{\partial \mathbf{f}}{\partial \omega}(\mathbf{x}, \omega)=\left(\begin{array}{llll}
-\frac{T^{2} \dot{y}}{2} & \frac{T^{2} \dot{x}}{2} & -T \dot{y} & T \dot{x}
\end{array}\right)^{\top} .
$$

The motion model developed in this section is used in the dolphin application as described in Application 3.2. A simulation of the model is also presented.

\section{Application 3.2: Dolphin Model}

The assumptions listed in Section 3.5.1 reasonably apply to dolphins swimming in a basin. Dolphins can swim in three dimensions, but the depth is less interesting in this application and difficult to measure, so only the two-dimensional movement is considered. Kolmården Wildlife Park has provided a scale drawing of the basin, from which the boundary of the basin is modelled as a polygon by selecting points along the edge of the basin in the map such that a reasonable approximation of its shape is obtained, see Figure 3.3.

Given that Assumption 3.1 holds, the model in (3.41) is used to model the motion of the dolphins. A simulation of a dolphin swimming according to this model is shown in Figure 3.3.

It should be noted that due to discretization of the continuous model in (3.37), there is still a chance that the simulation of dolphins at high speeds might end up outside the basin. To mitigate this problem, a higher sampling rate can be used or a test can be applied after each simulated step to verify that the constraints still hold and, if not, heuristically move the dolphin back inside the basin. If the basin is non-convex, there might also be a problem of an invisible basin edge causing strange behaviour.

The dolphins are observed through a fisheye camera with a highly nonlinear sensor model. The model for the camera is discussed in Section 4.1 and since this model is bijective, it is possible to pre-process a measurement such that a 

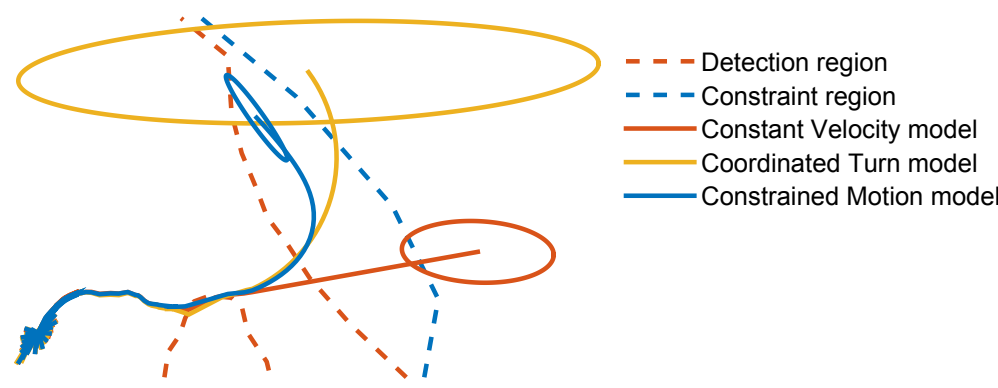

Figure 3.4: Compares the predictive capabilities and the measurement uncertainty of different models when a target stops generating measurements. The coordinated turn and constant velocity models do not take the constraint region into account resulting in infeasible predictions. The CMM keeps predictions within the constraint region. Published in [181].

two-dimensional position of the dolphin in world coordinates is obtained. The measurement noise is then modelled to be Gaussian in world coordinates rather than in camera coordinates, resulting in the sensor model

$$
\mathbf{y}_{j}=\mathbf{H} \mathbf{x}_{j}+\mathbf{v}_{j}=\left(\begin{array}{llll}
1 & 0 & 0 & 0 \\
0 & 1 & 0 & 0
\end{array}\right) \mathbf{x}_{j}+\mathbf{v}_{j}
$$

where only the position of the dolphin is observed and $\mathbf{v}_{j} \sim \mathcal{N}(\mathbf{0}, \mathbf{R})$. The position sensor model together with the CMM result in an AWGNSSM. See Application 3.3 for a comparison with conventional models.

A comparison between the proposed CMM and the conventional NCVM and Стм in the dolphin application is presented in Application 3.3.

\section{Application 3.3: Dolphin Model Comparison}

Conventional models do not take the physical constraints into account, which is why the смM is proposed. To show the differences in behaviour between the models, the prediction of each model with the resulting measurement uncertainty when no measurements are received is shown in Figure 3.4. The conventional models produce infeasible predictions and if the dolphin is rediscovered, due to a large gate or the prediction returning to the constraint region, the estimated trajectory is infeasible. The смм prediction, however, follows the boundary of the constraint region with a covariance better adapted to the actual uncertainty of the position. The uncertainty in the velocities is propagated to the uncertainty in the position, causing rapidly increasing uncertainty in the position for the conventional models, while the смм starts by increasing the uncertainty in position and then decreases it as the boundary is approached, although eccentricity still increases.

To improve the CMM further, the predictions from it could cover motions in both directions along a boundary until measurements have been acquired 
to distinguish which direction the dolphin chose. See Application 4.2 for the modelling of the camera used in the dolphinarium.

\subsection{Jump Markov Model}

A jump Markov model (JMM) [44] is useful when a target exhibits a few distinct behaviours or the type of target is unknown. A common example is found in tracking of vehicles, which often move relatively straight and make distinct turns every once in a while. For such targets, the first behaviour is modelled well by an NCVM and the second by a CTM. In Section 3.6.1 the model is described in general and in Section 3.6.2 an extension to the model is proposed by the author as Contribution IV, first published in [27]. Estimation methods for these models are presented in Section 5.4.

\subsubsection{General Model}

The JMм [44] considered is on the form

$$
\begin{aligned}
\mathbf{x}_{k} & =\mathbf{f}\left(\mathbf{x}_{k-1}, \delta_{k}\right)+\mathbf{w}_{k}, \\
\mathbf{y}_{j} & =\mathbf{h}_{j}\left(\mathbf{x}_{j}, \delta_{k}\right)+\mathbf{v}_{j}, \\
\mathbf{w}_{k} & \sim \mathcal{N}\left(\mathbf{0}, \mathbf{Q}_{k}\left(\delta_{k}\right)\right), \\
\mathbf{v}_{j} & \sim \mathcal{N}\left(\mathbf{0}, \mathbf{R}_{j}\left(\delta_{j}\right)\right), \\
p\left(\delta_{k} \mid \delta_{k-1}\right) & =\mathbf{\Pi}_{k}^{\delta_{k}, \delta_{k-1},}
\end{aligned}
$$

which is based on the AWGNSSM defined in (3.5)-(3.7). The AWGNSSM is extended with a discrete parameter $\delta_{k} \in \mathcal{S}$ representing the current mode of the system, where $\mathcal{S}$ is the set of modes. The prior probability at time step $k$ of transitioning from mode $a \in \mathcal{S}$ to mode $b \in \mathcal{S}$ is modelled by $\Pi_{k}^{b, a}$. The mode also exhibits the Markov property, resulting in a hidden Markov model for the mode. In many applications the main interest is the state, whereas in other applications the mode is more important.

The JMM is used to represent the different modes in the bird application as described in Application 3.4.

\section{Application 3.4: Bird Model}

The frame rate of the video is low and the volume within the funnel is small, making an NCPM sufficient. Although the funnel is three-dimensional, it is impossible with a single camera angle to reliably observe the bird's vertical position. The choice therefore lands on tracking the position of the bird in two dimensions and the state vector is chosen as in (3.18), where $x$ is the position in the magnetic east direction, and $y$ is the position in the magnetic north direction. The motion model is

$$
\mathbf{x}_{k}=\mathbf{x}_{k-1}+\mathbf{w}_{k}
$$


where $\mathbf{w}_{k} \sim \mathcal{N}\left(\mathbf{0}, T^{2} \tilde{\mathbf{Q}}\right), T$ is the inverse of the frame rate, and $\tilde{\mathbf{Q}}=\sigma^{2} \mathbf{I}$ determines the amount of movement of the bird.

The bird is observed through a camera, which is modelled as a perspective camera, and the sensor model used is on the form

$$
\mathbf{y}_{j}=\mathbf{h}\left(\mathbf{x}_{j}\right)+\mathbf{v}_{j},
$$

where the measurement function $\mathbf{h}\left(\mathbf{x}_{j}\right)$ is described in detail in Section 4.2 and $\mathbf{v}_{j} \sim \mathcal{N}(\mathbf{0}, \mathbf{R})$. The proposed sensor model together with the NCPM result in an AWGNSSM.

The bird is considered to have two distinct behaviours, stationary and flight, where the first denotes the behaviour of the bird being still and scanning with its head near the centre of the funnel and the latter denotes the behaviour of the bird fluttering its wings trying to escape the funnel. A JMM is therefore used with the two modes stationary and flight, denoted $s$ and $f$ respectively. For the modes $\delta_{k} \in \mathcal{S}$, where $\mathcal{S}=\{s, f\}$, (3.45) is modelled to match the behaviour of the bird as closely as possible.

Available measurements are the position of the bird in the image and the amount of blurriness in the vicinity of the bird. The position measurement is utilized when modelling the modes as an indication of the amount of movement of the bird. In the stationary mode, small movements are expected, which is modelled as a small covariance $\mathbf{Q}(s)$, and in the flight mode, large movements are expected, which is modelled as a large covariance $\mathbf{Q}(f)$. The blurriness measurement is utilized when modelling the modes as an indication of the amount of wing flutter. The measurement of blur depends on the background light, which changes over time, so the level of blur is modelled as a random walk for each mode. Augmenting the state vector in the NCPM with the blur level for each mode results in

$$
\mathbf{x}_{k}=\left(\begin{array}{c}
x_{k} \\
y_{k} \\
b_{k}^{s} \\
b_{k}^{f}
\end{array}\right)=\left(\begin{array}{c}
\mathbf{p}_{k} \\
b_{k}^{s} \\
b_{k}^{f}
\end{array}\right)
$$

where $\mathbf{p}_{k}$ is the position of the bird and $b_{k}^{f}$ and $b_{k}^{s}$ are the blurriness levels for the two modes respectively. Using the measurement model in (3.47) the augmented ssM becomes

$$
\begin{array}{rlrl}
\mathbf{x}_{k} & =\mathbf{x}_{k-1}+\mathbf{w}_{k}, & \mathbf{w}_{k} \sim \mathcal{N}\left(\mathbf{0}, \mathbf{Q}\left(\delta_{k}\right)\right), \\
\mathbf{y}_{k}=\left(\begin{array}{c}
\mathbf{h}\left(\mathbf{p}_{k}\right) \\
b_{k}^{\delta_{k}}
\end{array}\right)+\mathbf{v}_{k}, & \mathbf{v}_{k} \sim \mathcal{N}(\mathbf{0}, \mathbf{R}),
\end{array}
$$

with $\Pi^{\delta_{k}, \delta_{k-1}}$, covariances and prior distribution of the states tuned appropriately. The camera model is described in Application 4.1. 


\subsubsection{Model Extension}

In applications where the measurements are obtained through image processing, often an abundance of information can be extracted that might not directly relate to the state of the target, but might contain information directly dependent on the current mode. The model in (3.45) can then be extended with an additional measurement having the conditional distribution

$$
\mathbf{z}_{i} \sim p\left(\mathbf{z}_{i} \mid \delta_{i}\right), \quad i \in \mathcal{I} \subseteq \mathcal{K},
$$

where the conditional distribution is modelled to fit the application. Given the current mode $\delta_{k}$, the distribution is assumed to be conditionally independent from the states $\mathcal{X}_{1: k}$, the measurements $\mathcal{Y}_{1: k}$ and $\mathcal{Z}_{1: k-1}=\left\{\mathbf{z}_{i}\right\}_{i \in\{1: k-1\} \cap \mathcal{I}}$, and all previous modes $\Delta_{1: k-1}=\left\{\delta_{i}\right\}_{i=1}^{k-1}$ such that

$$
p\left(\mathbf{z}_{k} \mid \mathcal{Y}_{1: k}, \mathcal{Z}_{1: k-1}, \mathcal{X}_{1: k}, \Delta_{1: k}\right)=p\left(\mathbf{z}_{k} \mid \delta_{k}\right)
$$

The extension is used in the bird application as described in Application 3.5.

\section{- Application 3.5: Bird Model Extension}

Since the funnel forces the bird back into the centre when it is stationary, the radial distance of the bird from the centre can be seen as an indication of the current mode. This is used as an additional measurement in the JMM in Application 3.4. In practice the radial distance is generated from the current state $\mathbf{x}_{k}$ as

$$
z_{k}=\sqrt{x_{k}^{2}+y_{k}^{2}}
$$

but will, with a bit of abuse, be seen as a measurement from the image that is conditionally independent of the state given the current mode $\delta_{k}$. The distribution of $z_{k}$, given the current mode $\delta_{k}$, is modelled as

$$
p\left(z_{k} \mid \delta_{k}\right)= \begin{cases}c_{s} \cdot \mathcal{N}\left(z_{k} \mid 0, \sigma_{s}^{2}\right), & \text { if } 0 \leq z_{k} \leq r_{o} \text { and } \delta_{k}=s, \\ c_{f} \cdot \mathcal{N}\left(z_{k} \mid r_{o}, \sigma_{f}^{2}\right), & \text { if } 0 \leq z_{k} \leq r_{o} \text { and } \delta_{k}=f \\ 0, & \text { otherwise }\end{cases}
$$

where $r_{o}$ is the outer radius of the funnel, $c_{s}$ and $c_{f}$ are normalizing constants and $\sigma_{s}^{2}$ and $\sigma_{f}^{2}$ are design parameters.

\subsection{Extended Target Model}

Traditionally, the resolution in sensors has been so low that each target has been assumed to generate at most one measurement in each time step [11]. However, for many modern sensors the assumption no longer holds since targets may 
occupy multiple resolution cells in the sensor [77]. Commonly, such targets are referred to as extended.

One option is to perform significant amounts of pre-processing to extract a single detection per target, resulting in loss of information which might be of interest. Another option is to explicitly model the extension of the target to maintain the information.

Many options for modelling extended targets exist [20, 72, 78, 191]. In this thesis a model introduced in [107] is presented, where, in addition to the kinematic state vector, the extension is modelled as an ellipsoid represented by a random matrix. Although the exact shape of the target is not modelled this is sufficient for many applications [77]. An estimation method for the model introduced in this section is presented in Section 5.5.

Since a target can generate a number of measurements in each step, the set of measurements associated with the target is denoted

$$
\mathcal{Y}_{j}=\left\{\mathbf{y}_{j}^{i}\right\}_{i=1}^{N_{j}},
$$

where $N_{j}=\left|\mathcal{Y}_{j}\right|$ is the number of measurements associated with the target at time step $j$ and $\mathbf{y}_{j}^{i}$ is the $i$ th associated measurement at time step $j$. The measurements are assumed to be generated by independent and identically distributed processes. The SSM structures considered are a subset of LGSSMs including the motion models NCPM and NCVM. This is quite limiting, but still useful in many target tracking applications.

The measurement model is on the form

$$
p\left(\mathcal{Y}_{j} \mid N_{j}, \mathbf{x}_{j}, \mathbf{X}_{j}\right)=\prod_{i=1}^{N_{j}} \mathcal{N}\left(\mathbf{y}_{j}^{i} \mid\left(\tilde{\mathbf{H}}_{j} \otimes \mathbf{I}_{p}\right) \mathbf{x}_{j}, \mathbf{X}_{j}\right),
$$

where $\tilde{\mathbf{H}}_{j} \in \mathbb{R}^{1 \times s}$ is equal for all dimensions and $\mathbf{X}_{j} \in S_{++}^{p}$ is a symmetric positive definite random matrix that represents the extent of the target and $\mathbf{x}_{j} \in$ $\mathbb{R}^{s p}$. The implication of this model is that the measurement noise is replaced by the extent of the target. The motivation is that the measurement noise for high resolution sensors is insignificant compared to the extent of the target [107].

The motion model for the target is on the form

$$
p\left(\mathbf{x}_{k} \mid \mathbf{X}_{k}, \mathbf{x}_{k-1}\right)=\mathcal{N}\left(\mathbf{x}_{k} \mid\left(\tilde{\mathbf{F}}_{k} \otimes \mathbf{I}_{p}\right) \mathbf{x}_{k-1}, \tilde{\mathbf{Q}}_{k} \otimes \mathbf{X}_{k}\right),
$$

where $\tilde{\mathbf{F}}_{k}$ is equal for all dimensions. The motivation for including the extent in the process noise covariance is that oblong targets often move along their length, increasing uncertainty along the major axis.

By viewing the extent as the covariance in a Gaussian distribution, a straightforward choice for the distribution of the extent is the inverse-Wishart distribution, which is a conjugate prior distribution for the covariance matrix of a Gaussian [70]. The initial extension is then modelled as

$$
p\left(\mathbf{X}_{0}\right)=\mathcal{I} \mathcal{W}\left(\mathbf{X}_{0} \mid v_{0}, \mathbf{V}_{0}\right) \propto\left|\mathbf{X}_{0}\right|^{v_{0} / 2} \exp \left(-\frac{1}{2} \operatorname{tr}\left(\mathbf{V}_{0} \mathbf{X}_{0}^{-1}\right)\right)
$$


where $\mathbf{X}_{0} \in S_{++}^{p}$ and $\mathbf{V}_{0} \in S_{++}^{p}$ are symmetric positive definite matrices. The model for the prediction is chosen heuristically as

$$
p\left(\mathbf{X}_{k} \mid \mathbf{X}_{k-1}\right)=\mathcal{I} \mathcal{W}\left(\mathbf{X}_{k} \mid e^{-T_{k} / \tau} v_{k-1}, \frac{e^{-T_{k} / \tau} v_{k-1}-2 p-2}{v_{k-1}-2 p-2} \mathbf{V}_{k-1}\right),
$$

where the degrees of freedom decay with a time constant $\tau$, symbolizing increased uncertainty, and the expected value of the extent distribution,

$$
\mathbf{E}\left(\mathbf{X}_{k} \mid \mathbf{X}_{k-1}\right)=\frac{\mathbf{V}_{k}}{v_{k}-2 p-2}
$$

remains unaltered.

The extension model from this section is used with the NCVM in the savannah application as described in Application 3.6.

\section{Application 3.6: Savannah Animal Model}

The animals are tracked in image coordinates, so two dimensions for the positions are considered, resulting in a $p$ of 2 . The extended target model presented in this section is used to represent the targets. The quadcopter moves the camera in a relatively smooth manner, so an NCVM is a decent approximation of the animal movements, resulting in the motion model parameter

$$
\tilde{\mathbf{F}}_{k}=\left(\begin{array}{ll}
1 & T \\
0 & 1
\end{array}\right)
$$

in (3.56), where $T$ is the inverse of the camera frame rate. Since tracking is only performed in image coordinates, the measurement model parameter in (3.55) is

$$
\tilde{\mathbf{H}}_{j}=\left(\begin{array}{ll}
1 & 0
\end{array}\right) \text {. }
$$

The measurements $\mathcal{Y}_{j}$ are the pixels associated with a target in the image. See Application 4.6 for the method used to detect the savannah animals.

\subsection{Uncertain Timestamp Model}

Traditionally the timestamp of a measurement in an SSM is considered known and accurate. However, there are applications where this might not be the case. The uncertain timestamp model (UTM) was first proposed by the author in [182] to model such scenarios and further extended in [183]. The UTM is Contribution II of the thesis and is presented in this section. Measurements with precise timestamps are denoted type $\mathrm{M}$ measurements and observations with uncertain timestamps are denoted type o observations. Estimation methods for the UTM are proposed in Section 5.6.

The LGSSM in Section 3.2 is augmented with observations of type o, resulting in the UTM. Let $\mathcal{Z}=\left\{\mathbf{z}_{i}\right\}_{i \in \mathcal{I}}$ be the set of observations and $\mathcal{T}=\left\{\tau_{i}\right\}_{i \in \mathcal{I}}$ represent 
their timestamps, where $\mathcal{I}=\{1: I\}$. The observations $\mathbf{z}_{i} \in \mathbb{R}^{m_{z}}$ are modelled as independent conditioned on the states and the timestamps, and the model is given by

$$
\mathbf{z}_{i}=\mathbf{H}_{i}^{z} \mathbf{x}\left(\tau_{i}\right)+\mathbf{e}_{i}^{z}, \quad i \in \mathcal{I},
$$

where $\mathbf{H}_{i}^{z}$ is the observation matrix and $\mathbf{e}_{i}^{z} \sim \mathcal{N}\left(\mathbf{0}, \mathbf{R}_{i}^{z}\right)$ is noise. The timestamps are stochastic, but not necessarily independent, with a joint prior distribution modelled by

$$
\mathcal{T} \sim p(\mathcal{T})
$$

This distribution may, for example, be flat, given by context, obtained through measurements or estimated by the observer.

A one-dimensional version of the UTM with a single observation of type $o$ is used for analysing the impact of the additional observation in a simple uncertain time scenario as described in Application 3.7.

\section{Application 3.7: Simple Uncertain Timestamp Model}

The simple scenario in Application 2.4 is modelled as a UTM. An NCPM is used for the dynamical model on the form

$$
\begin{aligned}
d x(t) & =d \beta(t) & & E\left(d \beta^{2}(t)\right)=Q d t, \\
y_{1} & =x\left(t_{1}^{y}\right)+e_{1}^{y}, & & e_{1}^{y} \sim \mathcal{N}\left(0, R_{1}^{y}\right), \\
z_{1} & =x\left(\tau_{1}\right)+e_{1}^{z}, & & e_{1}^{z} \sim \mathcal{N}\left(0, R_{1}^{z}\right), \\
x(0) & \sim \mathcal{N}\left(\bar{x}_{0}, P_{0}\right), & &
\end{aligned}
$$

where the state $x(t)$ is the position, $\bar{x}_{0}=0 \mathrm{~km}, y_{1}=1 \mathrm{~km}$ and $t_{1}^{y}=1 \mathrm{~h}$. The specifications used are $Q=2.78 \cdot 10^{-3} \mathrm{~km}^{2} \mathrm{~h}^{-1}$ and $R_{1}^{y}=P_{0}=R_{1}^{z}=0.01 \mathrm{~km}^{2}$. Two different cases are considered with the following parameters:

1. $z_{1}=0.5 \mathrm{~km}$ and $p\left(\tau_{1}\right) \propto 1$.

2. $z_{1}=1.5 \mathrm{~km}$ and $p\left(\tau_{1}\right)=\mathcal{N}\left(\tau_{1} \mid 0.5,0.01\right)$.

The prior distribution of $\tau_{1}$ is uniformly distributed over the considered time interval between $0 \mathrm{~h}$ and $1 \mathrm{~h}$ in the first case, which is relatively uninformative, and is discretized uniformly in 600 steps for both cases. Using (3.14), the discretized matrices are computed as $F_{k}=1$ and $Q_{k}=Q \cdot\left(t_{k}-t_{k-1}\right)$ using (3.14). The posterior distribution for this model is derived in Application 5.2.

A two-dimensional version of the UTM is considered in a simulated scenario with two observations of type o and the model is described in Application 3.8. 


\section{Application 3.8: Simulated Uncertain Timestamp Model}

The purpose of this scenario is to demonstrate the effects of the timestamp uncertainty in two dimensions.

An NCVM is used on the form

$$
\begin{aligned}
d \mathbf{x}(t) & =\mathbf{A x}(t) d t+d \boldsymbol{\beta}(t), & & E\left(d \boldsymbol{\beta}(t) d \boldsymbol{\beta}(t)^{\top}\right)=\mathbf{Q} d t, \\
\mathbf{y}_{j} & =\mathbf{H x}\left(t_{j}^{y}\right)+\mathbf{e}_{j}^{y}, & & \mathbf{e}_{j}^{y} \sim \mathcal{N}\left(\mathbf{0}, \mathbf{R}^{y}\right), \\
\mathbf{z}_{i} & =\mathbf{H x}\left(\tau_{i}\right)+\mathbf{e}_{i}^{z}, & & \mathbf{e}_{i}^{z} \sim \mathcal{N}\left(\mathbf{0}, \mathbf{R}^{z}\right), \\
\mathbf{x}(0) & \sim \mathcal{N}\left(\overline{\mathbf{x}}_{0}, \mathbf{P}_{0}\right) & &
\end{aligned}
$$

where $\mathbf{y}_{j}$ with $\mathcal{T}_{y}=\{0: 6,12: 18,24: 30\} / 0.03 \mathrm{~s}$ for $j \in \mathcal{J}$ and $\mathbf{z}_{i}$ for $i \in \mathcal{I}=\{1,2\}$ are shown in Figure 2.6. The state vector $\mathbf{x}(t)$ consists of the two-dimensional position and velocity, $\mathbf{A}=\left(\begin{array}{ll}0 & 1 \\ 0 & 0\end{array}\right) \otimes \mathbf{I}_{2}$ and $\mathbf{H}=\left(\begin{array}{ll}1 & 0\end{array}\right) \otimes \mathbf{I}_{2}$. Two priors for the timestamps are considered, one with independent timestamps distributed uniformly for $i \in \mathcal{I}$ over the time interval $[0,1000] \mathrm{s}$ and another with similar joint distribution, but with the additional dependency that the timestamps are ordered such that $i>j$ implies that $\tau_{i}>\tau_{j}$. The specifications used are $\mathbf{Q}=\left(\begin{array}{cc}0 & 0 \\ 0 & \sigma^{2}\end{array}\right) \otimes \mathbf{I}_{2}, \sigma^{2}=5 \cdot 10^{-6} \mathrm{~m}^{2} \mathrm{~s}^{-3}, \mathbf{R}^{z}=5 \cdot 10^{-5} \cdot \mathbf{I}_{2} \mathrm{~m}^{2}$, $\mathbf{R}^{y}=5 \cdot 10^{-4} \cdot \mathbf{I}_{2} \mathrm{~m}^{2}, \overline{\mathbf{x}}_{0}=\left(0 \mathrm{~m}, 0 \mathrm{~ms}^{-1}\right)^{\top}$ and $\mathbf{P}_{0}=\operatorname{diag}\left(10^{9} \cdot \mathbf{I}_{2} \mathrm{~m}^{2}, \mathbf{I}_{2} \mathrm{~m}^{2} \mathrm{~s}^{-2}\right)$. Using (3.14), the discretized matrices are computed as $\mathbf{F}_{i, j}=\left(\begin{array}{cc}1 & T_{i, j} \\ 0 & 1\end{array}\right) \otimes \mathbf{I}_{2}$ and $\mathbf{Q}_{i, j}=\sigma^{2}\left(\begin{array}{cc}T_{i, j}^{3} / 3 & T_{i, j}^{2} / 2 \\ T_{i, j}^{2} / 2 & T_{i, j}\end{array}\right) \otimes \mathbf{I}_{2}$, where $T_{i, j}=t_{i}-t_{j}$.

The model in (3.65) is suitable for tracking where the control input is unknown, but to generate data that resembles a target following a desired trajectory a different approach is employed. The measurements $\mathcal{Y}$ are manually selected as a non-noisy version of $\mathcal{Y}$ in Figure 2.6. The posterior distribution for the model in (3.65) given only these measurements is computed. The timestamps are sampled from a distribution that is uniform in $[200,400] \mathrm{s}$ for $\tau_{1}$ and uniform in $[600,800] \mathrm{s}$ for $\tau_{2}$ to obtain one observation in each gap. A trajectory is sampled from the GP posterior distribution at the timestamps corresponding to $\mathcal{T}_{y}, \mathcal{T}$ and $\mathcal{T}_{p}$, where $\mathcal{T}_{p}$ is 200 equidistant samples in the interval $[0,1000] \mathrm{s}$. The measurements $\mathcal{Y}$ and observations $\mathcal{Z}$ are sampled from this realization according to the corresponding models in (3.65). One such realization is shown in Figure 2.6. The posterior and estimators are presented in Application 5.8.

A two-dimensional version of this model is used in the orienteering application and the model is described in Application 3.9.

\section{Application 3.9: Orienteering Uncertain Timestamp Model}

Without any information about the terrain that the sprinters move in, a reasonable assumption is that the sprinters take the shortest path at a stable pace to the next control. The sprinters are also assumed to remain on the ground, which is assumed to be flat. A two-dimensional NCVM is therefore suitable for the 
sprinters. The motion model is then given by

$$
\mathbf{x}_{k+1}=\mathbf{F} \mathbf{x}_{k}+\mathbf{w}_{k}, \quad k \in \mathcal{K}
$$

where the state vector is

$$
\mathbf{x}_{k}=\left(\begin{array}{llll}
x_{k} & y_{k} & \dot{x}_{k} & \dot{y}_{k}
\end{array}\right)^{\top},
$$

the transition matrix for the motion model is $\mathbf{F}=\left(\begin{array}{ll}1 & T \\ 0 & 1\end{array}\right) \otimes \mathbf{I}_{2}$ and the process noise $\mathbf{w}_{k} \sim \mathcal{N}(\mathbf{0}, \mathbf{Q})$ has the covariance $\mathbf{Q}=\sigma^{2}\left(\begin{array}{c}T^{3} / 3 T^{2} / 2 \\ T^{2} / 2\end{array}\right) \otimes \mathbf{I}_{2}$ where $T$ is the GNSS sampling time, and $\sigma^{2}$ determines how well the NCVM models the sprinter. The prior for the position is nearly flat and $v_{\max }^{2} / 3$ for the velocities.

The position of the sprinter is measured using a GNSS receiver. The GNSS is assumed to be biased by an offset due to occlusions, where the offset is modelled as a random walk. This results in an augmented state vector

$$
\mathbf{x}_{k}=\left(\begin{array}{llllll}
x_{k} & y_{k} & \dot{x}_{k} & \dot{y}_{k} & b_{k}^{x} & b_{k}^{y}
\end{array}\right)^{\top} .
$$

The transition model for the offset $\mathbf{b}_{k}=\left(b_{k}^{x}, b_{k}^{y}\right)^{\top}$ is

$$
\mathbf{b}_{k}=\alpha \mathbf{b}_{k-1}+\mathbf{w}_{k}^{b}, \quad \mathbf{w}_{k}^{b} \sim \mathcal{N}\left(\mathbf{0}, \mathbf{Q}^{b}\right),
$$

where the noise is independent and $\alpha \in[0,1]$ to make sure the offset returns to zero when no controls have been visited in a while. The prior for the offset is nearly flat. The sensor model for the GNSs measurements is

$$
\mathbf{y}_{k}=\mathbf{H}^{y} \mathbf{x}_{k}+\mathbf{v}_{k^{\prime}}^{y} \quad \mathbf{v}_{k}^{y} \sim \mathcal{N}\left(\mathbf{0}, \mathbf{R}^{y}\right), \quad k \in \mathcal{K}
$$

where

$$
\mathbf{H}^{y}=\left(\begin{array}{llllll}
1 & 0 & 0 & 0 & 1 & 0 \\
0 & 1 & 0 & 0 & 0 & 1
\end{array}\right)
$$

effectively selecting the sum of the position of the sprinter and the offset of the GNSS. The proposed sensor model together with the NCVM result in a LGSSM.

The observations, corresponding to the location of the controls, are modelled as

$$
\mathbf{z}_{i}=\mathbf{H}^{z} \mathbf{x}_{k_{i}}+\mathbf{v}_{i}^{z}, \quad \mathbf{v}_{i}^{z} \sim \mathcal{N}\left(\mathbf{0}, \mathbf{R}^{z}\right), \quad i \in \mathcal{I}
$$

where

$$
\mathbf{H}^{z}=\left(\begin{array}{llllll}
1 & 0 & 0 & 0 & 0 & 0 \\
0 & 1 & 0 & 0 & 0 & 0
\end{array}\right)
$$

effectively selecting the position of the sprinter. The prior on the timestamps $k_{i}$ is uniform but ordered, such that $i>j$ implies $k_{i}>k_{j}$, due to orienteering regulations. The results for the application are presented in Application 5.5. 


\subsection{Gaussian Process Motion Model}

The proposed Gaussian process motion model (GPMM) and a few variations are derived step-by-step in the following sections. First, linear Gaussian models with posterior distributions that can be computed analytically, since the input to the function is a known input to the model, are presented. Then an extension to a more useful nonlinear model where the input to the function depends on the state is provided. The GPMM is considered Contribution I and is under review for publication in [184]. Estimation methods for the GPMM are proposed in Section 5.7 .

\subsubsection{Dynamic System with Unknown Influence}

A linear Gaussian model of a dynamic system is considered,

$$
\begin{array}{rlrl}
\mathbf{x}_{0} & \sim \mathcal{N}\left(\overline{\mathbf{x}}_{0}, \mathbf{P}_{0}\right), & & \\
\mathbf{x}_{k} & =\mathbf{A}_{k} \mathbf{x}_{k-1}+\mathbf{B}_{k} \mathbf{f}_{k-1}+\mathbf{v}_{k}, & & k \in\{1: K\} \\
\mathbf{y}_{k} & =\mathbf{C}_{k} \mathbf{x}_{k}+\mathbf{e}_{k}, & & k \in\{1: K\} \\
\mathbf{f}_{k} & =\left(f^{1}\left(\mathbf{z}_{k}^{f}\right), \ldots, f^{J}\left(\mathbf{z}_{k}^{f}\right)\right)^{\top} & & \\
f^{j}(\mathbf{z}) & \sim \mathcal{G} \mathcal{P}\left(0, K\left(\mathbf{z}, \mathbf{z}^{\prime}\right)\right), & j \in\{1: J\}
\end{array}
$$

where $\mathbf{x}_{k} \in \mathbb{R}^{n}, \mathbf{y}_{k} \in \mathbb{R}^{m}$ and $\mathbf{z}_{k}^{f} \in \mathbb{R}^{p}$ are the state, the observation and the input, respectively, at time $k$. The input is known but influences the system in an unknown way, which is modelled by a function with a Gaussian process (GP) [151] prior, denoted $\mathcal{G} \mathcal{P}\left(\mu(\mathbf{z}), K\left(\mathbf{z}, \mathbf{z}^{\prime}\right)\right)$, with mean and covariance given by $\mu(\mathbf{z})$ and the kernel $K\left(\mathbf{z}, \mathbf{z}^{\prime}\right)$, respectively. The noise variables are independent and defined as $\mathbf{v}_{k} \sim \mathcal{N}\left(\mathbf{0}, \mathbf{Q}_{k}\right)$ and $\mathbf{e}_{k} \sim \mathcal{N}\left(\mathbf{0}, \mathbf{R}_{k}\right)$.

The notation $\mathbf{K}_{a b}$ will be used to denote the covariance matrix generated by the kernel $K\left(\mathbf{z}, \mathbf{z}^{\prime}\right)$ such that its elements are $\left[\mathbf{K}_{a b}\right]_{i l}=K\left(\mathbf{z}_{i}^{a}, \mathbf{z}_{l}^{b}\right)$, covering the range of indices $i$ and $l$ for the inputs. Similarly, the notation $\mathbf{K}_{a}$. (z) will be used to denote the column vector of cross-covariances such that $\left[\mathbf{K}_{a} .(\mathbf{z})\right]_{i}=K\left(\mathbf{z}_{i}^{a}, \mathbf{z}\right)$, and $\mathbf{K}_{\cdot a}(\mathbf{z})=\mathbf{K}_{a \cdot}^{\top}(\mathbf{z})$. Another shorthand is $\mathbf{K}_{a b}^{i}=\mathbf{K}_{\cdot b}\left(\mathbf{z}_{i}^{a}\right)$. Further, the notation $\tilde{\mathbf{A}}=\mathbf{A} \otimes \mathbf{I}_{J}$ will be used, where $\otimes$ denotes the Kronecker product and $\mathbf{I}_{a}$ denotes the $a \times a$ identity matrix.

Using the notation $\mathcal{F}=\left(\mathbf{f}_{1}^{\top}, \ldots, \mathbf{f}_{K}^{\top}\right)^{\top},(3.74 \mathrm{~d})-(3.74 \mathrm{e})$ can be replaced by $\mathcal{F} \sim$ $\mathcal{N}\left(\mathbf{0}, \tilde{\mathbf{K}}_{f f}\right)$. It can then be seen that the model in (3.74) is a regular LGSSM for which the posterior smoothing distribution can be computed analytically [159].

\subsubsection{Approximation of Influence Function}

By restricting the influence function kernel to use inducing variables, a recursive solution to the posterior filter distribution can be obtained [28]. The inducing inputs are fixed at $\mathbf{z}_{l}^{u}$, for $l \in\{1: L\}$ and the influence function values are given by the vector $\mathbf{u}_{l}=\left(f^{1}\left(\mathbf{z}_{l}^{u}\right), \ldots, f^{J}\left(\mathbf{z}_{l}^{u}\right)\right)^{\top}$ where $\mathcal{U}=\left(\mathbf{u}_{1}^{\top}, \ldots, \mathbf{u}_{L}^{\top}\right)^{\top}$. In [92] 
and [29] the inducing variables are used as states. Inspired by [148], $\mathcal{W}=$ $\left(\mathbf{w}_{1}^{\top}, \ldots, \mathbf{w}_{L}^{\top}\right)^{\top}=\tilde{\mathbf{K}}_{u u}^{-1} \mathcal{U}$ will be used as the states instead. The fully independent conditional (FIC) approximation [148] results in

$$
\left(\begin{array}{c}
\mathcal{F} \\
\mathcal{W}
\end{array}\right) \sim \mathcal{N}\left(\mathbf{0},\left(\begin{array}{cc}
\mathbf{Q}_{f f}+\boldsymbol{\Lambda} & \mathbf{K}_{f u} \mathbf{K}_{u u}^{-1} \\
\mathbf{K}_{u u}^{-1} \mathbf{K}_{u f} & \mathbf{K}_{u u}^{-1}
\end{array}\right) \otimes \mathbf{I}_{J}\right),
$$

where $\mathbf{Q}_{a b}=\mathbf{K}_{a u} \mathbf{K}_{u u}^{-1} \mathbf{K}_{u b}$ and $\boldsymbol{\Lambda}=\operatorname{diag}\left(\mathbf{K}_{f f}-\mathbf{Q}_{f f}\right)$.

The conditional distribution of $\mathcal{F}$ given $\mathcal{W}$ is

$$
\mathcal{F} \mid \mathcal{W} \sim \mathcal{N}\left(\tilde{\mathbf{K}}_{f u} \mathcal{W}, \tilde{\Lambda}\right)
$$

The joint distribution is then given by

$$
\begin{aligned}
p(\mathcal{Y}, \mathcal{X}, \mathcal{F}, \mathcal{W})=p(\mathcal{Y} \mid \mathcal{X}) p(\mathcal{X} \mid \mathcal{F}) p(\mathcal{F} \mid \mathcal{W}) p(\mathcal{W}) \\
=\prod_{k=1}^{K} \mathcal{N}\left(\mathbf{y}_{k} \mid \mathbf{C}_{k} \mathbf{x}_{k}, \mathbf{R}_{k}\right) \cdot \mathcal{N}\left(\mathbf{x}_{0} \mid \overline{\mathbf{x}}_{0}, \mathbf{P}_{0}\right) \cdot \mathcal{N}\left(\mathbf{x}_{k} \mid \mathbf{A}_{k} \mathbf{x}_{k-1}+\mathbf{B}_{k} \mathbf{f}_{k-1}, \mathbf{Q}_{k}\right) \cdot \\
\quad \mathcal{N}\left(\mathcal{F} \mid \tilde{\mathbf{K}}_{f u} \mathcal{W}, \tilde{\boldsymbol{\Lambda}}\right) \cdot \mathcal{N}\left(\mathcal{W} \mid \mathbf{0}, \tilde{\mathbf{K}}_{u u}^{-1}\right)
\end{aligned}
$$

Integration over $\mathcal{F}$ gives

$$
\begin{aligned}
\int_{\mathcal{F}} \prod_{k=1}^{K} \mathcal{N}\left(\mathbf{x}_{k} \mid \mathbf{A}_{k} \mathbf{x}_{k-1}+\mathbf{B}_{k} \mathbf{f}_{k-1}, \mathbf{Q}_{k}\right) \cdot \mathcal{N}\left(\mathcal{F} \mid \tilde{\mathbf{K}}_{f u} \mathcal{W}, \tilde{\Lambda}\right) d \mathcal{F} \\
\quad=\prod_{k=1}^{K} \int \mathcal{N}\left(\mathbf{x}_{k} \mid \mathbf{A}_{k} \mathbf{x}_{k-1}+\mathbf{B}_{k} \mathbf{f}_{k-1}, \mathbf{Q}_{k}\right) \cdot \mathcal{N}\left(\mathbf{f}_{k-1} \mid \tilde{\mathbf{K}}_{f u}^{k-1} \mathcal{W}, \tilde{\Lambda}_{k-1}\right) d \mathbf{f}_{k-1} \\
\quad=\prod_{k=1}^{K} \mathcal{N}\left(\mathbf{x}_{k} \mid \mathbf{A}_{k} \mathbf{x}_{k}+\mathbf{B}_{k} \tilde{\mathbf{K}}_{f u}^{k-1} \mathcal{W}, \mathbf{B}_{k} \tilde{\Lambda}_{k-1} \mathbf{B}_{k}^{\top}+\mathbf{Q}_{k}\right)
\end{aligned}
$$

which results in the marginalized joint distribution

$$
\begin{gathered}
p(\mathcal{Y}, \mathcal{X}, \mathcal{W})=\int_{\mathcal{F}} p(\mathcal{Y}, \mathcal{X}, \mathcal{F}, \mathcal{W}) d \mathcal{F}=\prod_{k=1}^{K} \mathcal{N}\left(\mathbf{y}_{k} \mid \mathbf{C}_{k} \mathbf{x}_{k}, \mathbf{R}_{k}\right) \cdot \mathcal{N}\left(\mathbf{x}_{0} \mid \overline{\mathbf{x}}_{0}, \mathbf{P}_{0}\right) \\
\prod_{k=1}^{K} \mathcal{N}\left(\mathbf{x}_{k} \mid \mathbf{A}_{k} \mathbf{x}_{k-1}+\mathbf{B}_{k} \tilde{\mathbf{K}}_{f u}^{k-1} \mathcal{W}, \mathbf{B}_{k} \tilde{\mathbf{\Lambda}}_{k-1} \mathbf{B}_{k}^{\top}+\mathbf{Q}_{k}\right) \cdot \mathcal{N}\left(\mathcal{W} \mid \mathbf{0}, \tilde{\mathbf{K}}_{u u}^{-1}\right) .
\end{gathered}
$$

The model can thus be rewritten in the form

$$
\begin{aligned}
\mathbf{x}_{0} & \sim \mathcal{N}\left(\overline{\mathbf{x}}_{0}, \mathbf{P}_{0}\right), \\
\mathbf{x}_{k} & =\mathbf{A}_{k} \mathbf{x}_{k-1}+\mathbf{B}_{k}\left(\tilde{\mathbf{K}}_{f u}^{k-1} \mathcal{W}+\mathbf{v}_{k}^{f}\right)+\mathbf{v}_{k}, \\
\mathbf{y}_{k} & =\mathbf{C}_{k} \mathbf{x}_{k}+\mathbf{e}_{k}, \\
\mathcal{W} & \sim \mathcal{N}\left(\mathbf{0}, \tilde{\mathbf{K}}_{u u}^{-1}\right),
\end{aligned}
$$


where $\mathbf{v}_{k}^{f} \sim \mathcal{N}\left(\mathbf{0}, \tilde{\Lambda}_{k}\right)$ and $\Lambda_{k}=[\boldsymbol{\Lambda}]_{k k}$. In case the subset of regressors (soR) approximation [148] is used let $\boldsymbol{\Lambda}=\mathbf{0}$, effectively eliminating $\mathbf{v}_{k}^{f}$ from the model. The model can be extended by including a prior mean $\overline{\mathcal{W}}_{0}=\tilde{\mathbf{K}}_{u u}^{-1} \overline{\mathcal{U}}_{0}$ of the function in (3.80d).

\subsubsection{Time-Varying Influence}

As noted in [191] and [166] the model in (3.80) allows the influence to be timevarying with a slight extension,

$$
\begin{aligned}
\mathbf{x}_{0} & \sim \mathcal{N}\left(\overline{\mathbf{x}}_{0}, \mathbf{P}_{0}\right), \\
\mathbf{x}_{k} & =\mathbf{A}_{k} \mathbf{x}_{k-1}+\mathbf{B}_{k}\left(\tilde{\mathbf{K}}_{f u}^{k-1} \mathcal{W}_{k-1}+\mathbf{v}_{k}^{f}\right)+\mathbf{v}_{k}, \\
\mathbf{y}_{k} & =\mathbf{C}_{k} \mathbf{x}_{k}+\mathbf{e}_{k}, \\
\mathcal{W}_{0} & \sim \mathcal{N}\left(\overline{\mathcal{W}}_{0}, \tilde{\mathbf{K}}_{u u}^{-1}\right), \\
\mathcal{W}_{k} & =\mathbf{G}_{k} \mathcal{W}_{k-1}+\mathbf{v}_{k}^{w},
\end{aligned}
$$

where $\mathbf{v}_{k}^{w} \sim \mathcal{N}\left(\boldsymbol{\mu}_{k}, \boldsymbol{\Sigma}_{k}\right)$. Influences, such as wind and currents, can thus be modelled to vary with time by introducing uncertainty in the form of process noise.

There are several options for $\mathbf{G}, \boldsymbol{\mu}_{k}$ and $\boldsymbol{\Sigma}_{k}$ that might be useful. In both [191] and [166] the transition matrix, mean and covariance are chosen as

$$
\begin{aligned}
\mathbf{G} & =e^{-\alpha T} \mathbf{I}, \\
\boldsymbol{\mu}_{k} & =\left(1-e^{-\alpha T}\right) \overline{\mathcal{W}}_{0}, \\
\boldsymbol{\Sigma}_{k} & =\left(1-e^{-2 \alpha T}\right) \tilde{\mathbf{K}}_{u u}^{-1},
\end{aligned}
$$

where $T$ is the sampling time and $\alpha$ is a forgetting factor determining the rate of convergence back to the prior distribution $\mathcal{W}_{0}$.

Another option is simply to let $\mathbf{G}=\mathbf{I}, \boldsymbol{\mu}_{k}=\mathbf{0}$ and $\boldsymbol{\Sigma}_{k}=\alpha \tilde{\mathbf{K}}_{u u}^{-1}$. The process will not converge to a stationary distribution, but the mean will remain unchanged, which occasionally could be desirable.

\subsubsection{Multiple Systems with Shared Influence}

In many scenarios multiple systems exhibit similar behaviour due to common influences from the surrounding environment, e.g. by wind, currents, paths and obstacles. Estimation of the common influence and the predictions of the systems are then improved by observing multiple systems.

The model in (3.81) is extended as

$$
\begin{aligned}
\mathbf{x}_{0}^{i} & \sim \mathcal{N}\left(\overline{\mathbf{x}}_{0}^{i}, \mathbf{P}_{0}^{i}\right), \\
\mathbf{x}_{k}^{i} & =\mathbf{A}_{k} \mathbf{x}_{k-1}^{i}+\mathbf{B}_{k}\left(\tilde{\mathbf{K}}_{i u}^{k-1} \mathcal{W}_{k-1}+\mathbf{v}_{k}^{i f}\right)+\mathbf{v}_{k^{\prime}}^{i}, \\
\mathbf{y}_{k}^{i} & =\mathbf{C}_{k} \mathbf{x}_{k}^{i}+\mathbf{e}_{k}^{i},
\end{aligned}
$$




$$
\begin{aligned}
& \mathcal{W}_{0} \sim \mathcal{N}\left(\overline{\mathcal{W}}_{0}, \tilde{\mathbf{K}}_{u u}^{-1}\right), \\
& \mathcal{W}_{k}=\mathbf{G}_{k} \mathcal{W}_{k-1}+\mathbf{v}_{k}^{w},
\end{aligned}
$$

where $\mathbf{z}_{k}^{i}$ is the input for process $i \in\{1: I\}$ and $\mathbf{v}_{k}^{i} \sim \mathcal{N}\left(\mathbf{0}, \mathbf{Q}_{k}^{i}\right), \mathbf{e}_{k}^{i} \sim \mathcal{N}\left(\mathbf{0}, \mathbf{R}_{k}^{i}\right)$ and $\mathbf{v}_{k}^{i f} \sim \mathcal{N}\left(\mathbf{0}, \tilde{\Lambda}_{k}^{i}\right)$ are noise variables with $\Lambda_{k}^{i}=\left[\mathbf{K}_{i i}-\mathbf{Q}_{i i}\right]_{k k}$. In the sequel the notation $\mathcal{X}_{k}=\left(\mathbf{x}_{k}^{1 \top}, \ldots, \mathbf{x}_{k}^{I \top}\right)^{\top}$ and $\mathcal{Y}_{k}=\left(\mathbf{y}_{k}^{1 \top}, \ldots, \mathbf{y}_{k}^{I \top}\right)^{\top}$ will be used. Although the processes are independent conditioned on the function, a dependence is introduced between the processes by the uncertainty of the function.

\subsubsection{State-Dependent Influence}

In many cases the inputs to the influence functions are not known inputs to the system, but depend on the state. The inputs are therefore modified to depend on the state as $\mathbf{z}_{k-1}^{i}=\mathbf{D}_{k} \mathbf{x}_{k-1}^{i}$. This model will be considered in the remainder of the thesis and is given by

$$
\begin{aligned}
\mathbf{x}_{0}^{i} & \sim \mathcal{N}\left(\overline{\mathbf{x}}_{0}^{i}, \mathbf{P}_{0}^{i}\right), \\
\mathbf{x}_{k}^{i} & =\mathbf{A}_{k} \mathbf{x}_{k-1}^{i}+\mathbf{B}_{k}\left(\tilde{\mathbf{K}}_{\cdot u}\left(\mathbf{D}_{k} \mathbf{x}_{k-1}^{i}\right) \mathcal{W}_{k-1}+\mathbf{v}_{k}^{i f}\left(\mathbf{D}_{k} \mathbf{x}_{k-1}^{i}\right)\right)+\mathbf{v}_{k}^{i}, \\
\mathbf{y}_{k}^{i} & =\mathbf{C}_{k} \mathbf{x}_{k}^{i}+\mathbf{e}_{k}^{i} \\
\mathcal{W}_{0} & \sim \mathcal{N}\left(\overline{\mathcal{W}}_{0}, \tilde{\mathbf{K}}_{u u}^{-1}\right), \\
\mathcal{W}_{k} & =\mathbf{G}_{k} \mathcal{W}_{k-1}+\mathbf{v}_{k}^{w},
\end{aligned}
$$

which is no longer linear. Note that $\mathbf{v}_{k}^{i f}(\mathbf{z}) \sim \mathcal{N}(\mathbf{0}, \tilde{\Lambda}(\mathbf{z}))$, where $\Lambda(\mathbf{z})=$ $K(\mathbf{z}, \mathbf{z})-\mathbf{K}_{\cdot u}(\mathbf{z}) \mathbf{K}_{u u}^{-1} \mathbf{K}_{u} \cdot(\mathbf{z})=K(\mathbf{z}, \mathbf{z})-\sum_{l=1}^{L} \sum_{l^{\prime}=1}^{L} K\left(\mathbf{z}, \mathbf{z}_{l}^{u}\right)\left[\mathbf{K}_{u u}^{-1}\right]_{l l^{\prime}} K\left(\mathbf{z}_{l^{\prime}}^{u}, \mathbf{z}\right)$. Letting $i=1$ a model for a single dynamical system is obtained as a special case.

\subsubsection{Covariance Function for Influence}

The choice of covariance function $K\left(\mathbf{z}, \mathbf{z}^{\prime}\right)$ is important as it encodes the assumptions made on the function to be learnt [191]. There are many covariance functions to consider, see e.g. [151], which additionally depend on hyperparameters that need to be selected carefully. The hyperparameters are either selected manually using expert knowledge or are learned from the data [29, 92, 125, 151], where the latter is often preferred, but results in additional steps that may be complicated depending on the problem at hand.

For simplicity, the squared-exponential kernel, given by

$$
K\left(\mathbf{z}, \mathbf{z}^{\prime}\right)=\sigma^{2} \exp \left(-\frac{1}{2 \theta^{2}}\left\|\mathbf{z}-\mathbf{z}^{\prime}\right\|^{2}\right),
$$

is used in the remainder of the thesis and the hyperparameters are selected manually. The hyperparameter $\theta$ works as a length scale for the covariance function 
inputs, modelling the smoothness of the function, and $\sigma^{2}$ determines the variance of the function, modelling the belief in the prior mean. The estimation approach considered also requires the derivative of the covariance function, which is given by

$$
\mathbf{K}_{\mathbf{z}^{\prime}}\left(\mathbf{z}, \mathbf{z}^{\prime}\right) \triangleq \frac{\partial}{\partial \mathbf{z}^{\prime}} K\left(\mathbf{z}, \mathbf{z}^{\prime}\right)=\frac{K\left(\mathbf{z}, \mathbf{z}^{\prime}\right)}{\theta^{2}}\left(\mathbf{z}-\mathbf{z}^{\prime}\right)^{\top}=-\mathbf{K}_{\mathbf{z}}\left(\mathbf{z}, \mathbf{z}^{\prime}\right),
$$

where the notation $\mathbf{A}_{\mathbf{a}}(\mathbf{b})=\partial \mathbf{A}(\mathbf{a}) /\left.\partial \mathbf{a}\right|_{\mathbf{a}=\mathbf{b}}$ is used.

A simulation of multiple targets is performed in Application 3.10 using the GPMM proposed in this section.

\section{Application 3.10: Velocity Field Model}

To demonstrate and evaluate the proposed method a tracking scenario is simulated based on the model in Section 3.9.5. The underlying model for each target is given by

$$
\begin{aligned}
& \mathbf{x}_{k}=\mathbf{x}_{k-1}+T \cdot \mathbf{f}\left(\mathbf{x}_{k-1}\right)+\mathbf{v}_{k}, \\
& \mathbf{y}_{j}=\mathbf{x}_{k_{j}}+\mathbf{e}_{j},
\end{aligned}
$$

where the state $\mathbf{x}_{k}$ is the position at time step $k \in\{1: K\}, \mathbf{y}_{j}$ for $j \in\{0:\lfloor K / S\rfloor\}$ is an observation of the position at time step $k_{j}=j S, S$ determines time between observations and $\mathbf{f}(\mathbf{x})$ determines the velocity of a target given its location. The velocity function $\mathbf{f}(\mathbf{x})$ is shared between all targets. The observation and process noises are given by $\mathbf{e}_{j} \sim \mathcal{N}(\mathbf{0}, \mathbf{R})$ with covariance $\mathbf{R}=9 \mathbf{I}_{2} \mathrm{~m}^{2}$ and $\mathbf{v}_{k} \sim \mathcal{N}(\mathbf{0}, \mathbf{Q})$ with covariance $\mathbf{Q}=q^{2} T \mathbf{I}_{2}$, respectively, where $T=1 \mathrm{~s}$ is the discretization time. The function, shown in Figure 2.9, is continuous and designed so that the targets roughly follow a path, which, e.g., could represent a racing track. A sample of 200 targets are drawn uniformly and simulated $150 \mathrm{~s}$ according to the model. The environment is assumed to be clutter free and correct associations are assumed to be given. Data is generated with the parameters $q \in\{0,0.5,2,5,20,50\} \mathrm{m} / \sqrt{\mathrm{s}}$ and $S \in\{1,2,5,10\}$ in various configurations. A Monte Carlo simulation is performed where a first realization is used to optimize the hyperparameters for each model and another 100 realizations are used for estimation.

The model used for estimation is

$$
\begin{aligned}
\mathbf{x}_{0}^{i} & \sim \mathcal{N}\left(\overline{\mathbf{x}}_{0}^{i}, \mathbf{P}_{0}\right), \\
\mathbf{x}_{k}^{i} & =\mathbf{x}_{k-1}^{i}+T \cdot\left(\tilde{\mathbf{K}}_{\cdot u}\left(\mathbf{x}_{k-1}^{i}\right) \mathcal{W}+\mathbf{v}_{k}^{i f}\left(\mathbf{x}_{k-1}^{i}\right)\right)+\mathbf{v}_{k}^{i}, \\
\mathbf{y}_{j}^{i} & =\mathbf{x}_{k_{j}}^{i}+\mathbf{e}_{j}^{i}, \\
\mathcal{W} & \sim \mathcal{N}\left(\mathbf{0}, \tilde{\mathbf{K}}_{u u}^{-1}\right),
\end{aligned}
$$

where the function is modelled as time-invariant, index $i$ has been added to reference the target, and the covariance function is chosen as in (3.85). The inducing points are gridded in the visible part of Figure 2.9 with a spacing given 
by $\delta$. For comparison, the NCVM and NCAM are considered, with the respective process covariances $\mathbf{Q}_{v}$ and $\mathbf{Q}_{a}$ given by the parameters $q_{v}^{2}$ and $q_{a}^{2}$.

The true measurement noise covariance matrix $\mathbf{R}$ is used and the process noise covariances $\mathbf{Q}_{v}$ and $\mathbf{Q}_{a}$ are optimized over $q_{v} \in\{0.05,0.5,5,50\} \mathrm{m} / \sqrt{\mathrm{s}}^{3}$ and $q_{a} \in\{0.05,0.5,5,50\} \mathrm{m} / \sqrt{\mathrm{s}}^{5}$, respectively. For the model in (3.88) the true $\mathbf{R}$ and $\mathbf{Q}$ are used and the hyperparameters are optimized over $\theta \in\{25,50,75\} \mathrm{m}$, $\sigma^{2} \in\{1,10,50\} \mathrm{m}^{2} / \mathrm{s}^{2}$ and $\delta \in\{50,75\} \mathrm{m}$. The state prior is the observation for the position and mean zero for the other states with covariance $\mathbf{P}_{0}=\mathbf{R}$ for the proposed model, $\mathbf{P}_{0}=\operatorname{diag}\left(\mathbf{R}, 25 \cdot \mathbf{I}_{2} \mathrm{~m} / \mathrm{s}\right)$ for the $\mathrm{NCVM}$ and $\mathbf{P}_{0}=$ $\operatorname{diag}\left(\mathbf{R}, 25 \cdot \mathbf{I}_{2} \mathrm{~m} / \mathrm{s}, 25 \cdot \mathbf{I}_{2} \mathrm{~m}^{2} / \mathrm{s}^{2}\right)$ for the NCAM. The results for the scenario are presented in Application 5.10.

The GPMM is applied in the sea ice tracking application. The model is presented in Application 3.11.

\section{Application 3.11: Sea Ice Learning Model}

The ice objects are assumed to have independent velocities, but are affected by a common acceleration from currents and winds, which are subject to change over time. The model for the ice objects used for estimation is therefore chosen as

$$
\begin{aligned}
\mathbf{x}_{k_{1}^{i}}^{i} & \sim \mathcal{N}\left(\overline{\mathbf{x}}_{0}^{i}, \mathbf{P}_{0}\right), \\
\mathbf{x}_{k}^{i} & =\mathbf{A} \mathbf{x}_{k-1}^{i}+\mathbf{B}\left(\tilde{\mathbf{K}}_{\cdot u}\left(\mathbf{D} \mathbf{x}_{k-1}^{i}\right) \mathcal{W}_{k}+\mathbf{v}_{k}^{i f}\left(\mathbf{D} \mathbf{x}_{k-1}^{i}\right)\right)+\mathbf{v}_{k^{\prime}}^{i} \\
\mathbf{y}_{j}^{i} & =\mathbf{C} \mathbf{x}_{k_{j}^{i}}^{i}+\mathbf{e}_{j}^{i}, \\
\mathcal{W}_{0} & \sim \mathcal{N}\left(\mathbf{0}, \tilde{\mathbf{K}}_{u u}^{-1}\right), \\
\mathcal{W}_{k} & =e^{-\alpha T} \mathcal{W}_{k-1}+\mathbf{v}_{k}^{w},
\end{aligned}
$$

where the state $\mathbf{x}_{k}^{i}$ is the position and the velocity of ice object $i \in\{1: I\}$ at time step $k \in\left\{k_{1}^{i}, \ldots, k_{J}^{i}\right\}$ and $k_{j}^{i}$ denotes the time step of observation $j \in\{1: J\}$ for the target. The kernel is chosen as in (3.85) and $\mathbf{v}_{k}^{w} \sim \mathcal{N}\left(\mathbf{0},\left(1-e^{-2 \alpha T}\right) \tilde{\mathbf{K}}_{u u}^{-1}\right)$ with $T=0.05 \mathrm{~h}$. The system matrix is $\mathbf{A}=\left(\begin{array}{ll}1 & T \\ 0 & 1\end{array}\right) \otimes \mathbf{I}_{2}, \mathbf{B}=\left(\begin{array}{c}T^{2} / 2 \\ T\end{array}\right) \otimes \mathbf{I}_{2}, \mathbf{v}_{k}^{i} \sim$ $\mathcal{N}\left(\mathbf{0}, \mathbf{Q}_{v}\right)$ with $\mathbf{Q}_{v}$ defined as in (3.22) and the matrix $\mathbf{D}$ selects the position states. The observation noise is given by $\mathbf{e}_{j}^{i} \sim \mathcal{N}\left(\mathbf{0}, r^{2} \mathbf{I}_{2}\right)$ and the observation matrix $\mathbf{C}$ selects the position states. The state prior is the first observation for the position and zero velocity with $\mathbf{P}_{0}=\operatorname{diag}\left(r^{2} \mathbf{I}_{2}, 500^{2} \mathbf{I}_{2} \mathrm{~m}^{2} / \mathrm{h}^{2}\right)$. For comparison, the NCVM and NCAM are also considered with the respective covariance matrices $\mathbf{Q}_{v}$ in (3.22) and $\mathbf{Q}_{a}$ in (3.23). The method of extracting measurements from the scans is described in Applications 4.7 and 4.4. 


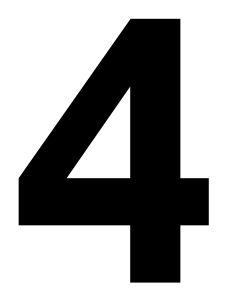

\section{Camera Sensors}

Cameras are versatile sensors with applications in a wide range of areas. In this thesis mainly stationary cameras are considered. They have fixed position, fixed angle and fixed field of view relative to the scene. The main advantage is that each pixel then corresponds to a fixed ray through the scene, allowing for relatively simple detection of movement. Camera modelling is important in computer vision and more details on the subject are provided in, e.g., [86, 117].

Common components of a camera model are described in Section 4.1. In Section 4.2 models for stationary cameras are considered and model estimation is discussed for the models. In Section 4.3 a common method for background estimation using stationary cameras is presented. A slight modification is proposed, which was first published by the author in [181], and a discussion is presented on rather heuristic approaches for foreground segmentation that can be applied for non-stationary cameras. A method for clustering and mapping pixels through nonlinear camera models is proposed in Section 4.4, first presented by the author in [181].

\subsection{Camera Model}

There has been much research into computer vision in the last few decades, a field based on the camera as a versatile sensor. To infer knowledge related to world coordinates from camera images, a calibration of the camera is needed. Calibration is the estimation of a model relating world coordinates to pixels in the image recorded by the camera. Such models and their estimation are briefly described in this section. For a more thorough overview of computer vision and reconstruction from images see, e.g., [3, 117].

Given a number of world coordinates $\mathbf{x}_{i}^{r}=\left(x_{i}^{r}, y_{i}^{r}, z_{i}^{r}\right)^{\top}$ and corresponding image coordinates $\mathbf{x}_{i}^{c}=\left(x_{i}^{c}, y_{i}^{c}\right)^{\top}$ of a set of points, $i \in\{1: M\}$, one wants to 
find a mapping, or camera model, $\mathbf{x}^{c}=\mathbf{h}\left(\mathbf{x}^{r}\right)$ between the points. The mapping is in general nonlinear, but due to the geometry and physical properties of the camera a parametric model can be constructed to simplify the problem of calibrating the camera. A common method is to decompose the model into the components [117]:

- camera extrinsics corresponding to the rotation and translation of the camera in world coordinates;

- a projection from three dimensions to two dimensions;

- camera intrinsics corresponding to field of view, principal point and pixel sizes;

- perspective compensation; and

- lens distortion.

One reason for this decomposition is that camera extrinsics might change dynamically while other components are camera dependent and do not change for a fixed focal length. To simplify the notation homogeneous coordinates [117] are used, obtained by augmenting a coordinate vector by an element of one, denoted $\tilde{\mathbf{a}}=\left(\mathbf{a}^{\top}, 1\right)^{\top}$ for an arbitrary vector $\mathbf{a}$ in the sequel.

Camera extrinsics, projection, camera intrinsics and perspective compensation are given by

$$
\lambda \tilde{\mathbf{x}}^{u}=\mathbf{K} \Pi_{0} \mathbf{G} \tilde{\mathbf{x}}^{r},
$$

where $\mathbf{x}^{u}$ is the undistorted coordinate, $\lambda$ compensates for the perspective and ensures homogeneous coordinates, and the projection is given by

$$
\Pi_{0}=\left(\begin{array}{llll}
1 & 0 & 0 & 0 \\
0 & 1 & 0 & 0 \\
0 & 0 & 1 & 0
\end{array}\right) .
$$

Camera intrinsics are modelled by

$$
\mathbf{K}=\left(\begin{array}{ccc}
\alpha_{x} & \gamma & p_{x} \\
0 & \alpha_{y} & p_{y} \\
0 & 0 & 1
\end{array}\right)
$$

where $\alpha_{x}=f \sigma_{x}$ and $\alpha_{y}=f \sigma_{y}$ model the focal length $f$ and pixel sizes $\sigma_{x}$ and $\sigma_{y}, \gamma$ models pixel skewness, and $\mathbf{p}=\left(p_{x}, p_{y}\right)^{\top}$ is the principal point. See Figure 4.1 for an illustration of a projection onto the image plane for a scene already transformed into camera coordinates. Camera extrinsics are modelled by

$$
\mathbf{G}=\left(\begin{array}{ll}
\mathbf{R} & \mathbf{T} \\
\mathbf{0} & 1
\end{array}\right)
$$




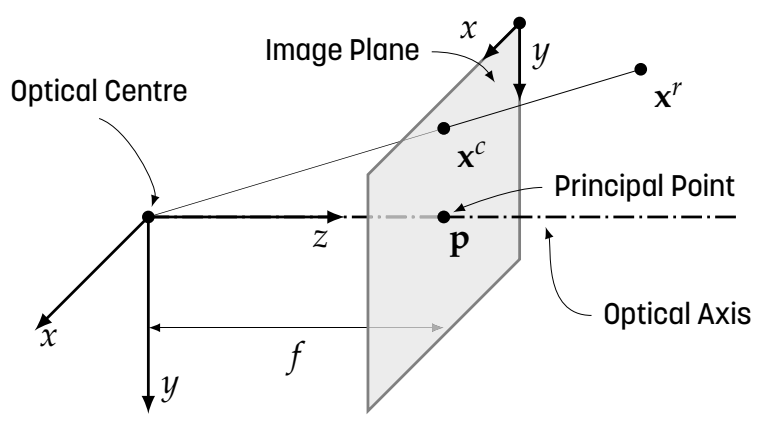

Figure 4.1: Illustration of the projection and the role of the focal length and principal point in camera calibration. The point $\mathbf{x}_{r}$ in the coordinate system of the camera is projected onto the image plane for a camera with focal length $f$ and principal point

p.

where $\mathbf{R} \in S O(3)$ is a rotation matrix and $\mathbf{T} \in \mathbb{R}^{3}$ is a translation vector, mapping world coordinates into the coordinate system of the camera.

In case the lens distortion is minimal, a decent model is $\mathbf{x}^{c}=\mathbf{x}^{u}$, however, for wide angle lenses the distortion can be significant and is compensated for by a nonlinear transformation, $\mathbf{x}^{c}=\mathbf{f}\left(\mathbf{x}^{u}\right)$. The structure and parametrization of the nonlinearity mainly depend on the type of distortion in the lens. A polynomial in the distance from the centre of distortion is suggested in [117] for radial distortion on the form

$$
\mathbf{x}^{c}=\mathbf{x}_{o}+\left(1+a_{1} r+a_{2} r^{2}+a_{3} r^{3}+\ldots\right)\left(\mathbf{x}^{u}-\mathbf{x}_{o}\right),
$$

where $r=\left\|\mathbf{x}^{u}-\mathbf{x}_{o}\right\|$ is the distance from the pixel to the centre of distortion $\mathbf{x}_{o} \in \mathbb{R}^{2}$ that might be different from the principal point. Both the centre and the coefficients in the polynomial need to be estimated for the lens. This is a general model with many parameters, so for small $M$ a less flexible structure with fewer parameters might be preferred.

The process of estimating the parameters in $\mathbf{K}, \mathbf{G}$ and $\mathbf{f}(\mathbf{x})$ is called camera calibration and is often essential for tracking using cameras. Estimation of the parameters is quite involved, however, and algorithms are described in [117, 201]. An implementation of the algorithms is available as open source [34]. A straightforward approach is to grab frames from the camera viewing a checker board pattern from different angles, making all the parameters, as well as the camera extrinsics, identifiable. This is not possible in all cases, which then requires a tailored solution for the setup. One such approach is described in Section 4.2.

\subsection{Stationary Camera Model}

A complete calibration of the camera is not always possible, either because the camera is no longer available or it is mounted in a location that is difficult to 
reach. Even if it were available, the mounted position of the camera in world coordinates might be difficult to estimate, reducing the usefulness of a traditional calibration. A different approach might therefore be more suitable in such cases.

For a stationary camera, the camera extrinsics never change. The first three factors on the right hand side of (4.1) are therefore constant and the resulting matrix $\mathbf{H}=\mathbf{K} \Pi_{0} \mathbf{G}$ can be considered directly, reducing (4.1) to

$$
\lambda \tilde{\mathbf{x}}^{u}=\mathbf{H} \tilde{\mathbf{x}}^{r} .
$$

If an object in the video with distinguished features can be identified and has known dimensions in world coordinates, the model in (4.6) can be estimated directly using, e.g., a trust-region algorithm [39]. It is also possible to determine constraints on $\mathbf{H}$ in (4.6) for the estimation by deriving them from the camera model in (4.1).

In the bird application the dimensions of each funnel are known and the circles at the top and bottom are identified in the image. The circles are then used for camera calibration in Application 4.1.

\section{Application 4.1: Bird Camera}

The camera is mounted above four funnels placed in a square looking straight down as shown in Figure 2.2 and the vertical axis in the image is aligned with the magnetic field, with north towards the top of the image. The origins in world coordinates are placed at the centres of the funnels with north along the $y$-axis and the $z$-axis pointing out of the top of the funnels. This implies a rotation $\mathbf{R}=\operatorname{diag}(1,-1,-1)$ of the camera, representing a half revolution around the world $x$-axis, and an unknown translation $\mathbf{T}$. Furthermore, square pixels are assumed, reducing (4.3) to

$$
\mathbf{K}=\left(\begin{array}{ccc}
\alpha & 0 & p_{x} \\
0 & \alpha & p_{y} \\
0 & 0 & 1
\end{array}\right)
$$

which results in a parametrization $\mathbf{p}=\left(p_{1}, \ldots, p_{6}\right)^{\mathrm{T}}$ of $\mathbf{H}$ as

$$
\mathbf{H}=\left(\begin{array}{cccc}
p_{1} & 0 & p_{2} & p_{3} \\
0 & -p_{1} & p_{4} & p_{5} \\
0 & 0 & -1 & p_{6}
\end{array}\right)
$$

The lens distortion is assumed minimal and is modelled as $\mathbf{x}^{\mathcal{c}}=\mathbf{x}^{u}$.

The dimensions of a funnel are given in world coordinates as the inner radius $r_{i}=125 \mathrm{~mm}$, the outer radius $r_{o}=325 \mathrm{~mm}$ and the height $h=155 \mathrm{~mm}$. Although a slight distortion occurs in the transformation, the inner and outer circles of the funnel are estimated approximately as circles directly from the video. By identifying coordinates on the form $\left(x^{\mathcal{C}}, y^{\mathcal{c}}\right)^{\top}$ along the inner and outer circles a nonlinear least-squares estimate [138] is obtained for the parameters $\bar{x}, \bar{y}$ and $r$ of each circle represented on the form

$$
\left(x^{c}-\bar{x}\right)^{2}+\left(y^{c}-\bar{y}\right)^{2}=r^{2} .
$$


Matching coordinates to use for the calibration are then obtained by equiangularly sampling $M$ coordinates along the inner and outer circles in two dimensions for the image and in three dimensions for the funnel. The coordinates are represented as

$$
\begin{aligned}
\mathbf{x}_{i}^{c}=\left(\begin{array}{l}
x_{i}^{c} \\
y_{i}^{c}
\end{array}\right), & i \in\{1: M\}, \\
\mathbf{x}_{i}^{r}=\left(\begin{array}{l}
x_{i}^{r} \\
y_{i}^{r} \\
z_{i}^{r}
\end{array}\right), & i \in\{1: M\},
\end{aligned}
$$

where $z_{i}^{r}=-h / 2$ for the inner circle and $z_{i}^{r}=h / 2$ for the outer circle.

The perspective compensation makes the mapping defined in (4.6) nonlinear, so the trust-region algorithm described in [39] is used to solve for the unknown parameters $\mathbf{p}$ in (4.8). The model in (4.6) is used to derive the measurement function $\mathbf{h}(\mathbf{x})$ from world coordinates to image coordinates in (3.47).

The position of the bird along the $z$-axis in world coordinates cannot be reliably estimated using a single camera sensor, so the vertical coordinate of the bird is fixed to the wall of the funnel at all times, which means that the $z$-coordinate is computed as

$$
z^{r}=-\frac{h}{2}+h \cdot \min \left(\max \left(\sqrt{\left(x^{r}\right)^{2}+\left(y^{r}\right)^{2}}, r_{O}\right), r_{I}\right) /\left(r_{O}-r_{I}\right) .
$$

The resulting mapping for one of the funnels is shown in Figure 4.2. See Application 4.5 for a description of the algorithm used to detect the birds.

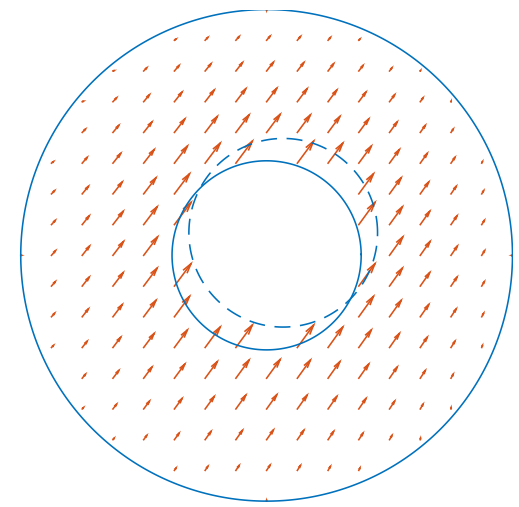

Figure 4.2: The funnel from above in world coordinates and scaled and translated image coordinates. The solid blue lines show the inner and outer circle in world coordinates and the dashed blue line shows the inner circle in image coordinates for comparison. The red arrows show the mapping for different parts of the funnel. 
A blueprint of the basin in the dolphin application is available, see Figure 3.3, and points are manually matched between the blueprint and the camera image, see Figure 2.1. The matched points are used to calibrate the camera in Application 4.2.

\section{Application 4.2: Dolphin Camera with Fisheye Lens}

A camera with a fisheye lens, which exhibits severe radial distortion, is used for monitoring the dolphins as a solution to local regulations concerning audience integrity. It is mounted in the ceiling of the dolphinarium with most of the basin in its field of view, but not with complete coverage. The blueprint is planar, so without loss of generality the surface of the basin is chosen as reference height, reducing the problem to finding a mapping between two planes. With abuse of notation, the world coordinates $\mathbf{x}^{r}$ and $\mathbf{H}$ in (4.6) are for this example redefined as

$$
\begin{aligned}
\mathbf{x}^{r} & =\left(\begin{array}{l}
x^{r} \\
y^{r}
\end{array}\right), \\
\mathbf{H} & =\left(\begin{array}{lll}
H_{11} & H_{12} & H_{13} \\
H_{21} & H_{22} & H_{23} \\
H_{31} & H_{32} & H_{33}
\end{array}\right) .
\end{aligned}
$$

This simplification effectively assumes that everything seen in the camera exists at the surface of the water in the basin. Matrix $\mathbf{K}$ in (4.3) is always full rank considering $\alpha_{x} \neq 0$ and $\alpha_{y} \neq 0$, and the matrix $\Pi_{0} \mathbf{G}$ given by (4.2) and (4.4) with the third column removed is full rank as long as the translation is out of the plane spanned by the $x$ - and $y$-axes in world coordinates, which is the case for the camera mounted in the ceiling. Thus, a properly estimated matrix $\mathbf{H}$ will be invertible. The mapping between undistorted image and world coordinates is therefore bijective, allowing also undistorted image coordinates to be mapped directly into world coordinates.

A distortion model also needs to be estimated for the fisheye lens. As discussed in Section 4.1, it is common to use a polynomial distortion model, but [54] suggests the following model for fisheye lenses

$$
\begin{aligned}
& r^{c}=R\left(r^{u}\right)=\frac{1}{\omega} \arctan \left(2 r^{u} \tan \frac{\omega}{2}\right), \\
& r^{u}=R^{-1}\left(r^{c}\right)=\frac{\tan \left(r^{c} \omega\right)}{2 \tan \frac{\omega}{2}},
\end{aligned}
$$

where $r^{\mathcal{C}}=\left\|\mathbf{x}^{\mathcal{C}}-\mathbf{x}_{o}\right\|$ is the radial distance from the centre of distortion $\mathbf{x}_{o}$ in the image, $r^{u}=\left\|\mathbf{x}^{u}-\mathbf{x}_{o}\right\|$ is the radial distance in the undistorted image, and $\omega$ is a parameter determining the amount of distortion. The mapping is bijective and computed as

$$
\begin{aligned}
& \mathbf{x}^{c}=\mathbf{x}_{o}+\frac{R\left(r^{u}\right)}{r^{u}}\left(\mathbf{x}^{u}-\mathbf{x}_{o}\right), \\
& \mathbf{x}^{u}=\mathbf{x}_{o}+\frac{R^{-1}\left(r^{c}\right)}{r^{c}}\left(\mathbf{x}^{c}-\mathbf{x}_{o}\right) .
\end{aligned}
$$




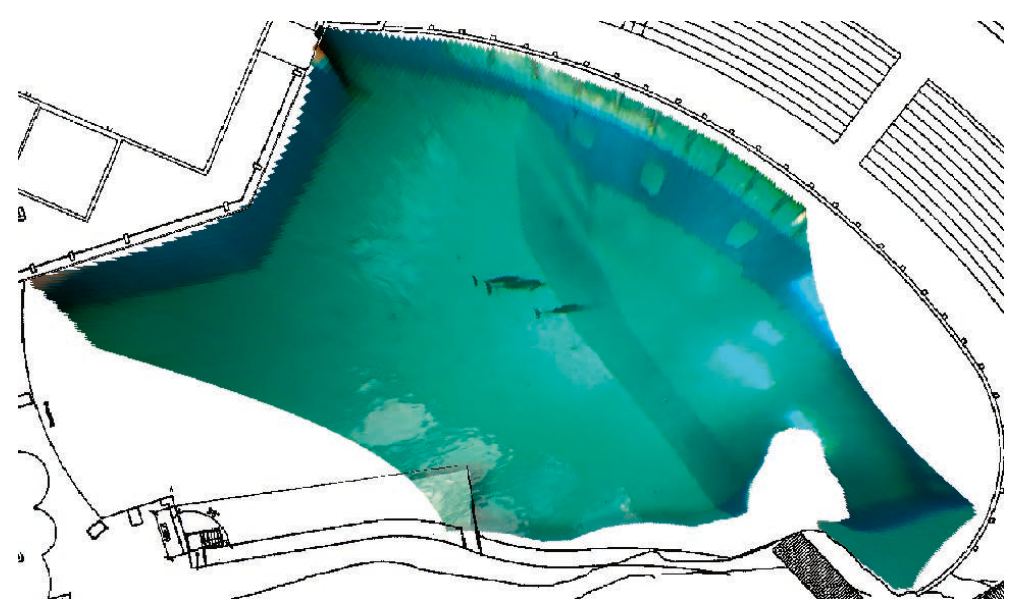

Figure 4.3: A video frame from the camera mounted in the dolphinarium. The image has been inverted using the estimated camera model and drawn into the map of the basin in world coordinates.

The method described in [202] is used to estimate $\mathbf{H}$ in (4.12b) by finding the linear least-squares solution as an initial guess and refining it with a trustregion method [39]. The parameters $\omega$ and $\mathbf{x}_{o}$ are also estimated using the trustregion method. These solutions are computed in an alternating manner until convergence is achieved to find the complete mapping, as suggested by [202].

Having estimated the model parameters, $(4.12 \mathrm{~b})$ and $(4.14 \mathrm{~b})$ are used to derive a measurement function from world coordinates to image coordinates. Further, since the mapping is static and bijective, the inverse function can also be used to map coordinates in the camera directly into world coordinates.

In Figure 4.3 a frame from the video has been inverted using the inverse mapping from image coordinates to world coordinates and drawn into the map of the basin. The mapping is not perfect in all parts of the image, but many issues caused by the fisheye lens have been mitigated. See Application 4.3 for a description of the foreground segmentation used to detect the dolphins.

\subsection{Foreground Segmentation}

To obtain detections of objects in the video it is segmented into background and foreground. For stationary cameras, a background model can be estimated over time for each pixel using, e.g., a mixture of Gaussians as in [147, 167] or quantiles as described in [38]. Estimation of a background model is difficult for nonstationary cameras. As an alternative, standard image processing methods, see e.g. [75], can be tailored to the particular application to estimate the background and to extract detections of the target. 


\subsubsection{Gaussian Mixture Model}

For stationary cameras, a Gaussian-mixture background model [147, 167] is described in this section with a few modifications proposed by the author. The basic idea is to estimate mixtures of Gaussians to represent the pixel intensities using the expectation-maximization (EM) algorithm [53]. Considering the large number of pixels, several approximations of this algorithm are applied to make computations less demanding. The intensities of a new image are gated and associated to the Gaussian components of the model. If an association is found, the model is updated, otherwise a new Gaussian is initialized with low weight. Gaussian components with large weights are considered background whereas those with small weights are considered to be foreground.

A single channel is considered for each pixel, resulting in one-dimensional Gaussian components. The Gaussian-mixture background model for each pixel consists of $K$ components with mean $\mu_{j}$, variance $\sigma_{j}^{2}$ and weight $w_{j}, j \in\{1$ : $K\}$, sorted such that $w_{j} / \sigma_{j}$ is in descending order. The first $K_{B}$ components are considered background, where $K_{B}$ is determined as the smallest value such that $\sum_{j=1}^{K_{B}} w_{j}>T$, where $T \in[0,1]$ is a threshold. Note that most variables in this section implicitly depend on the time and the pixel, but that these dependences are not reflected explicitly unless necessary.

The parameter $\gamma^{2}$ determines the maximum squared Mahalanobis distance $d_{j}(I)$ considered a match through the criterion

$$
d_{j}(I)=\frac{\left(I-\mu_{j}\right)^{2}}{\sigma_{j}^{2}} \leq \gamma^{2},
$$

where $I$ is the currently measured intensity for the pixel. Selecting $\gamma^{2}$ is a tradeoff between tolerating variations in the background and detecting foreground. According to [167], it can be advantageous to replace $\gamma^{2}$ by $\gamma_{k}^{2}$, explicitly showing the dependence on time step $k$, allowing the threshold to vary over time and different regions in the scene.

If no $j \leq K_{B}$ exists such that $d_{j}(I) \leq \gamma^{2}$ in (4.15), the pixel is considered to be part of the foreground. If no match is found at all, the last component is replaced by a new component with a mean at $I$ and default initial values for variance and weight. If a match is found for component $j$, its mean, variance and weight are updated as

$$
\begin{aligned}
P_{j} & =w_{j} \mathcal{N}\left(I \mid \mu_{j}, \Sigma_{j}\right), \\
w_{j}^{+} & =w_{j}+\alpha\left(P_{j}-w_{j}\right), \\
\rho_{j} & =\frac{\alpha P_{j}}{w_{j}^{+}}, \\
\mu_{j}^{+} & =\mu_{j}+\rho_{j}\left(I-\mu_{j}\right), \\
\Sigma_{j}^{+} & =\Sigma_{j}+\rho_{j}\left(\left(I-\mu_{j}\right)^{2}-\Sigma_{j}\right),
\end{aligned}
$$


where the superscript + denotes the updated values and $\alpha$ is a parameter that determines the learning speed. For all other components, the weights are reduced by a factor $1-\alpha$. The weights are then divided by the sum of all weights for renormalization after each update.

A proposed extension to the method is to also compute

$$
d=\min _{j \leq K_{B}} d_{j}(I),
$$

for each foreground pixel, providing the Mahalanobis distance to the nearest background component. This value provides information about the degree of confidence for the detection, which could allow for more sophisticated methods in the post-processing to globally segment the foreground or improve the tracking as in [35], but is here used to weight individual measurements as described in Section 4.4. Additional extensions, such as using track predictions as feedback in the foreground segmentation, can be considered to improve the performance, e.g., as suggested by [171].

The foreground segmentation is generally noisy and is filtered using morphological operations [75], after which the output is $M_{f}$ observations consisting of the coordinates $\breve{\mathbf{y}}_{i}$ of the foreground pixels and their values $d_{i}$ obtained from (4.17). The set is denoted

$$
\breve{\mathcal{Y}}=\left\{\breve{\mathbf{y}}_{i}, d_{i}\right\}_{i=1}^{M_{f}} \text {. }
$$

The method is used to extract detections of the dolphins in a challenging environment as described in Application 4.3.

\section{Application 4.3: Dolphin Background Estimation}

The measurement pre-processing in the dolphin application aims at segmenting the foreground in the video. The camera is stationary, but the scene suffers from sunlight through large skylights in the dolphinarium causing changes in the ambient light, reflections in the water and shadows at the bottom of the basin. To handle such dynamic light conditions, the method of estimating a Gaussianmixture background model is applied as described in this section. The model is well suited for estimating the reflections in the background. This either results in several background components or an increased variance of a single component.

The method, as described, assumes an image with a single channel, which is obtained as a function of the red, green and blue channels. The function is chosen to achieve a reduction in the variance of the background pixels. Furthermore, the mean scene intensity is subtracted to make the model less sensitive to the light conditions.

To further improve robustness against changing light conditions, the threshold $\gamma_{k}^{2}$ is selected using the heuristics

$$
\gamma_{k}^{2}=\gamma_{0}^{2}+\gamma_{g}^{2} \max _{s \in[k-\tau, k]} \sqrt{\left|\bar{I}_{s}-\bar{I}_{s-1}\right|}
$$

where $\tau, \gamma_{0}^{2}$ and $\gamma_{g}^{2}$ are design parameters and $\bar{I}_{k}$ is the mean intensity in the image at time $k$. The second term increases the tolerance for all pixels when 


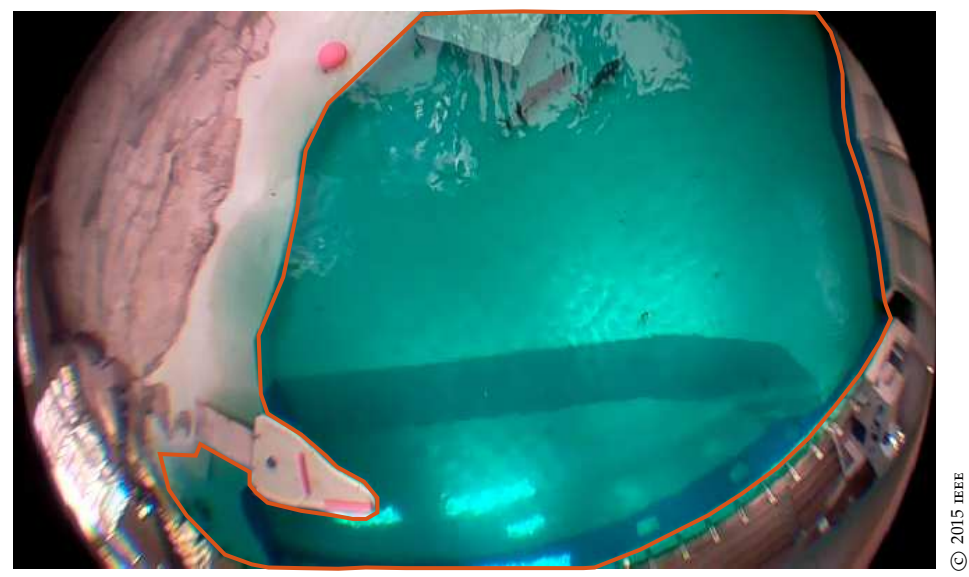

Figure 4.4: The detection region in the video, displayed as a red polygon, is the region of visible water in the basin where dolphins can be detected. Published in [181].

the light conditions in the scene change drastically for a time determined by $\tau$, allowing the current background components to adapt to the new conditions rather than to estimate new background components, which otherwise would result in many false detections.

To improve the computational performance, a detection region is defined in the image in the form of a polygon, selecting the visible part of the water in the basin where dolphins can possibly be detected. The detection region is shown in Figure 4.4. Since each pixel is modelled independently, the uneven shape of the region has only a limited effect on the result.

Each pixel is fed into the background estimation algorithm. Initially all pixels will be considered background by the algorithm, but after a number of iterations in a training period, the moving dolphins will be detected as foreground.

The extension described in (4.17) is used. It not only returns the estimated foreground, but also the statistical distance to the nearest background component. Examples of segmented targets are shown in Figure 4.5. The quality of the output varies over the region and over time depending on, e.g., the stability of the background, separation of targets, camera resolution and distortion and light conditions. Although more information could be extracted using tailored computer vision techniques, thresholding together with morphological operations is sufficient for the intended target tracking and to use standard methods is beneficial when applying the same solution to similar problems. See Application 4.8 for a method to reduce the large number of pixels detected for the dolphins. 


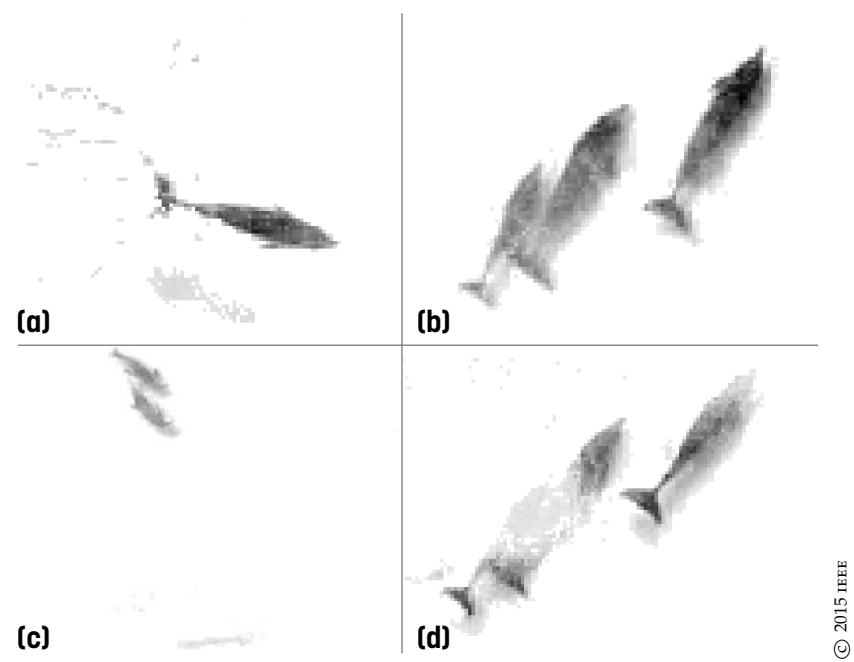

Figure 4.5: Shows the Mahalanobis distance output for segmented pixels in the foreground segmentation for four situations: (a) a properly segmented target together with noise and a prominent shadow, (b) three separate dolphins where the two on the left would be combined by thresholding, (c) a faint dolphin at the bottom, (d) dolphins partly disappearing in a reflective region. Published in [181].

Segmentation of drift ice with low signal-to-noise ratio that requires pre-processing over several scans for detection is described in Application 4.4. A land mask is applied to the image to ensure that detections are only obtained in water regions.

\section{Application 4.4: Drift Sea Ice Segmentation}

Drift ice is generally small and often difficult to distinguish in the speckle noise. A Gaussian mixture background model is recursively estimated in water regions as presented in this section. For an incoming scan, all pixels that are significantly lighter than their background models are segmented as foreground. This implies many false detections, which is mitigated by extracting connected components of a minimum size of $150 \mathrm{~m}^{2}$. The detections are then obtained as the centroid of each connected component. The drift-ice detections are shown in red in Figure 4.6. The method applied to detect stationary ice is described in Application 4.7.

\subsubsection{Moving Quantile Model}

Another approach is to model the background as moving quantiles [38]. This method is used for the bird application as outlined in Application 4.5. 


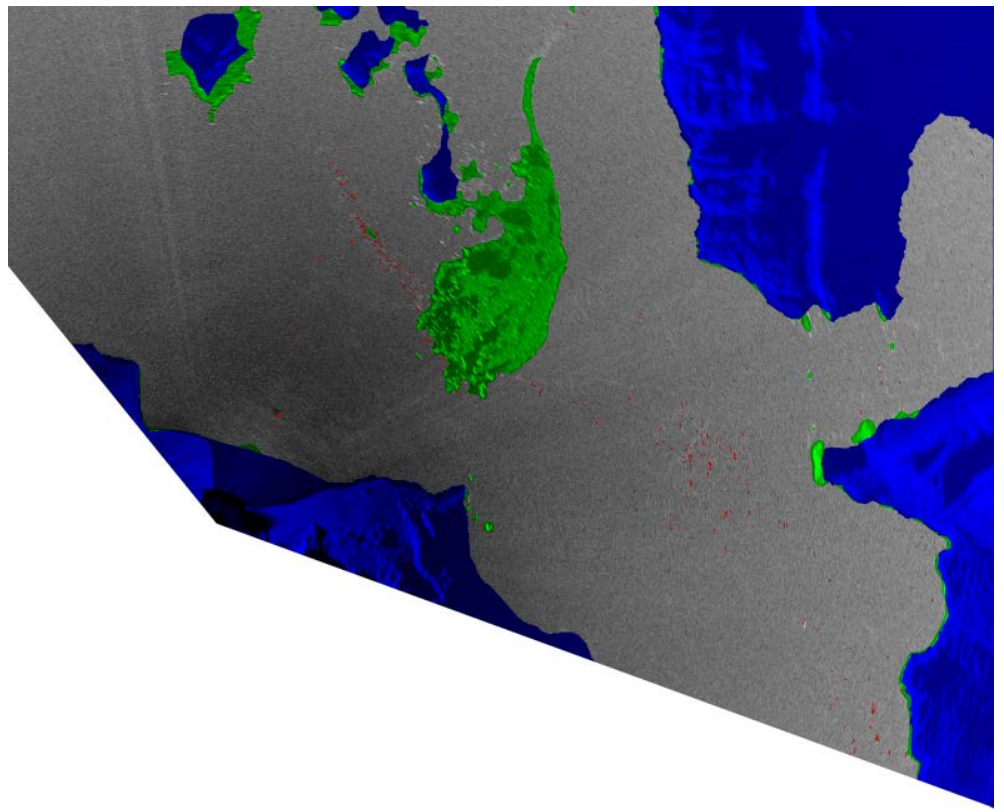

Figure 4.6: A single scan showing an overlay of the land mask in blue, stationary ice in green and drift ice detections in red.

\section{Application 4.5: Bird Background Estimation}

The background estimation in the bird application was provided by Dr. Kristoffer Öfjäll at Computer Vision Laboratory, Linköping University. The method used is presented in more detail in the appendix of [27]. A moving quantile model [38] is used for each pixel and an ellipse around the bird is estimated using channel decoding as described in [63]. This provides a measurement of the position and orientation of the bird. Wing flutter is then estimated as the amount of motion blur in foreground pixels. An example of a frame with the estimated background subtracted is shown in Figure 4.7. The results of the solution are presented in Application 5.1.

\subsubsection{Methods for Nonstationary Cameras}

For nonstationary cameras, estimating the background over time is a much more challenging task. With significant amounts of prior information, however, detections can instead be extracted using image processing methods tailored to the particular application. General image processing methods that can be applied are described in, e.g., [75]. Such a method is designed for the savannah application as presented in Application 4.6. 


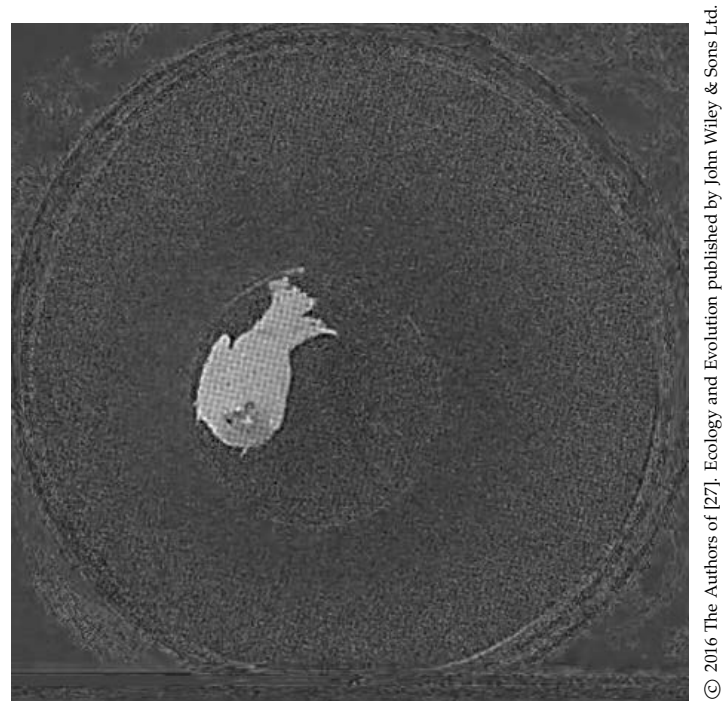

Figure 4.7: Example of a pre-processed image frame where the bird has been enhanced significantly by subtracting the estimated background. Published in [27].

\section{Application 4.6: Savannah Animal Segmentation}

The camera in the Savannah application is non-stationary, so estimating the background over time is a difficult task. A simple heuristic method to estimate the background in a single frame is to apply the morphological operation open [75] with a structure larger than the expected size of the targets. The background is then subtracted from the original image, allowing for variations in the background over the scene. The estimated background is shown in Figure 4.8.

Computing the gradient [75] and adding to the image improves the contrast between the targets and the background, as is shown in Figure 4.9. A threshold is then applied to the image and the connected components [75] are extracted. All components with at least one pixel at a higher threshold are then extracted as detections, see Figure 4.10. The results for the ETT solution are presented in Application 6.1.

In the sea ice tracking application the sensor is stationary. The aim is to process the signal in order to maximize the likelihood that the resulting detections are generated by true sea ice. Two types of ice are considered, stationary ice adhering to land masses and individual ice objects drifting in the sea.

Large regions of stationary sea ice with high signal-to-noise ratio that can be segmented independently for each scan as described in Application 4.7. 


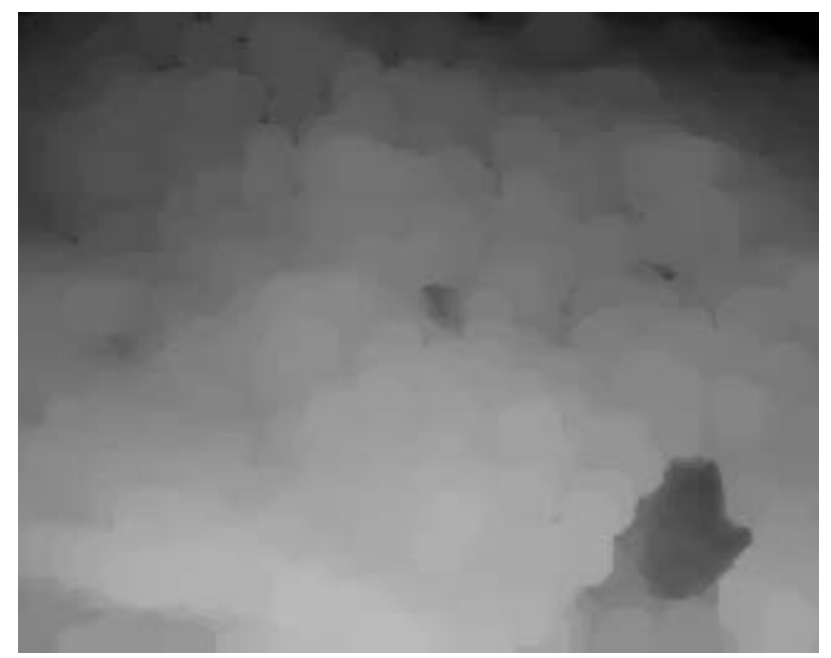

Figure 4.8: The estimation of the background using morphological operations for a single frame in the savannah application.

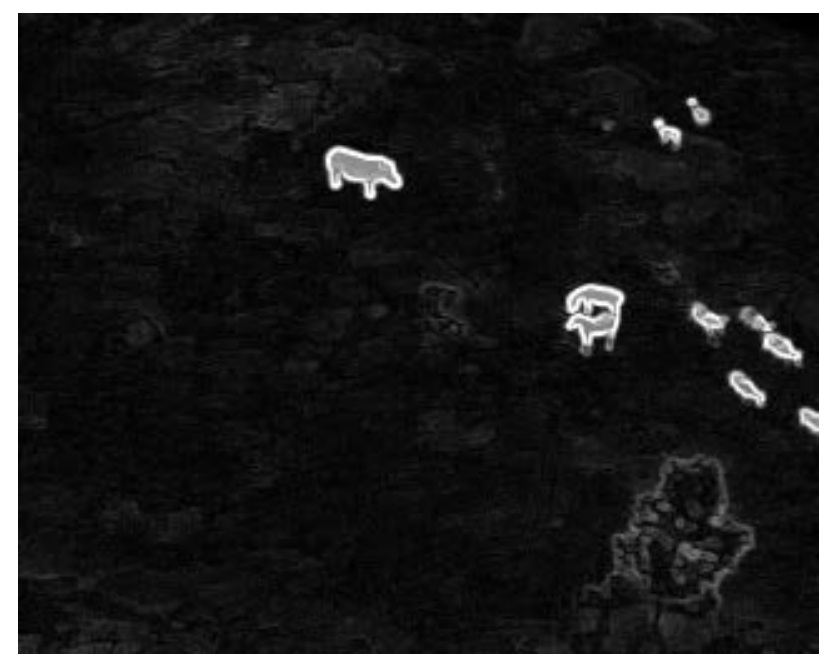

Figure 4.9: A single frame after background subtraction and gradient addition in the savannah application. 


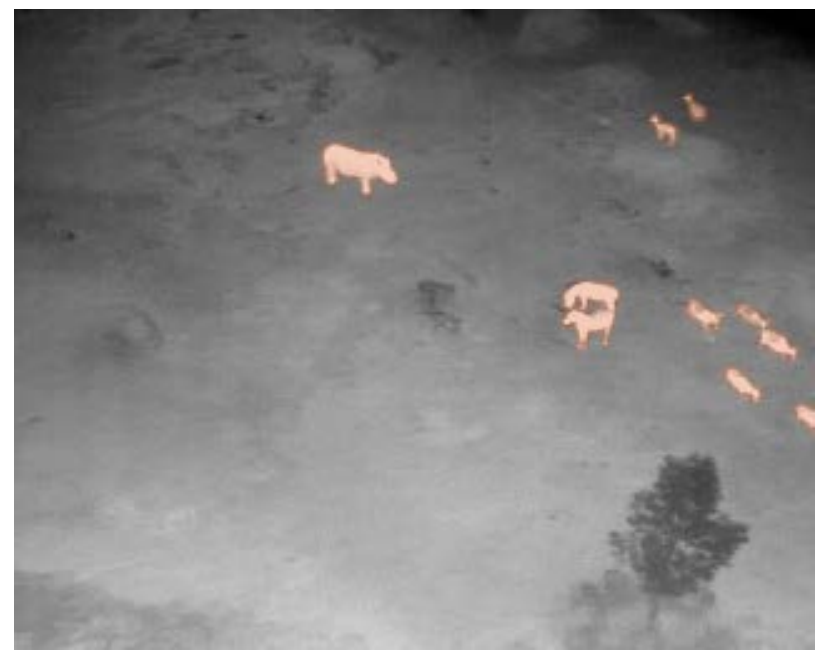

Figure 4.10: A single frame with detections marked in red in the savannah application.

Application 4.7: Stationary Sea Ice Segmentation

Large areas of stationary ice are visible in the water, in particular in proximity to land. However, due to speckle noise and varying intensity over the image a simple threshold results in poor performance. To improve the signal-to-noise ratio, a sequence of standard image segmentation methods [75] are applied.

The stationary regions are first stabilized by averaging the ten latest frames. A Gaussian filter and a top-hat transform are then applied to reduce speckle noise and intensity variations in the image, respectively [75]. The segmentation of the stationary ice regions is performed using Otsu's optimal thresholding [143]. To further reduce noise in the segmentation, closing and hole filling are also applied [75]. The connected components adjacent to land are finally extracted as detections of stationary sea ice regions. The stationary sea ice regions are shown in green in Figure 4.6. The results for the sea ice tracking and learning applications are presented and discussed in Applications 6.4 and 5.11, respectively.

\subsection{Data Reduction}

There are multiple detections per target, which might be computationally expensive for a target tracking filter to process. In this section a method to reduce the amount of data is proposed by the author.

A first step is to obtain the indices $i$ of connected components $\tilde{\mathcal{C}_{l}}, l \in\{1: \tilde{M}\}$, from the measurements $\breve{\mathbf{y}}_{i}$ in (4.18) using the flood fill algorithm [75]. 
The second step is to use the $k$-means clustering algorithm [118] on the measurements $\left\{\breve{\mathbf{y}}_{i}\right\}_{i \in \tilde{\mathcal{C}}_{l}}$ for each connected component to obtain the clusters $\mathcal{C}_{l}^{m}$, $m \in\left\{1: M_{l}\right\}$. To obtain clusters of approximate size $m_{r}$ the number of clusters for each component is chosen as $\left[\left|\tilde{\mathcal{C}}_{l}\right| / m_{r}\right]$, where $|\mathcal{A}|$ denotes the cardinality of set $\mathcal{A}$ and $\lceil a\rceil$ denotes the ceiling of $a$.

To reduce the number of measurements, the means are computed as

$$
\overline{\mathbf{y}}_{l}^{m}=\frac{1}{\left|\mathcal{C}_{l}^{m}\right|} \sum_{i \in \mathcal{C}_{l}^{m}} \breve{\mathbf{y}}_{i} \quad \text { and } \quad \bar{d}_{l}^{m}=\frac{1}{\left|\mathcal{C}_{l}^{m}\right|} \sum_{i \in \mathcal{C}_{l}^{m}} d_{i} .
$$

To keep partial information regarding the extent of the connected component the covariance of the measurements is computed as

$$
\overline{\mathbf{Y}}_{l}^{m}=\frac{1}{\left|\mathcal{C}_{l}^{m}\right|} \sum_{i \in \mathcal{C}_{l}^{m}}\left(\breve{\mathbf{y}}_{i}-\overline{\mathbf{y}}_{l}^{m}\right)\left(\breve{\mathbf{y}}_{i}-\overline{\mathbf{y}}_{l}^{m}\right)^{\top},
$$

and a reduced measurement set is obtained as

$$
\overline{\mathcal{Y}}=\bigcup_{l=1}^{\tilde{M}} \bigcup_{m=1}^{M_{l}}\left\{\left(\overline{\mathbf{y}}_{l}^{m}, \bar{d}_{l}^{m}, \overline{\mathbf{Y}}_{l}^{m}\right)\right\},
$$

with cardinality $M=|\overline{\mathcal{Y}}|$.

To exactly map the ellipsoids represented by the means and covariances in the reduced measurement set in (4.22) using the nonlinear camera model in Section 4.2 is nontrivial. Since approximations have already been introduced, the extent is approximated using the unscented transform [100] of (4.22). The sigma-points are mapped using a camera model and recomputed centroids and covariances are obtained. The measurements are also reindexed, resulting in the mapped reduced measurement set

$$
\mathcal{Y}=\left\{\left(\mathbf{y}_{i}, d_{i}, \mathbf{Y}_{i}\right)\right\}_{i=1}^{M}
$$

The method is applied in Application 4.8 to the resulting set of measurements in Application 4.3.

\section{Application 4.8: Dolphin Data Reduction}

An example of the segmented foreground for two dolphins, obtained as described in Application 4.3, is shown in Figure 4.11 together with the reduced measurements $\overline{\mathcal{Y}}$. The resulting mapped measurements $\mathcal{Y}$ are shown in Figure 4.12. See Application 6.2 for a comparison between two tracking filters using the reduced data set. 
Figure 4.11: The segmented foreground is in grey scale, where a darker shade indicates a higher degree of confidence. On top are green ellipsoids representing the extents of the clusters, and a darker shade indicates a higher degree of confidence.

Figure 4.12: The extent of each cluster is mapped to world coordinates using an unscented transform and the camera model in Application 4.2. The shape has been distorted and scaled compared to Figure 4.11. 



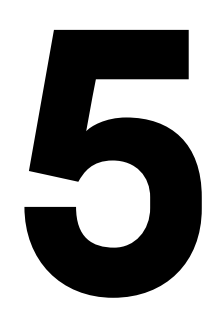

\section{Estimation}

Given a model and measurements one wants to estimate the states and possibly other parameters in the model. This chapter presents theory on estimation for the models introduced in Chapter 3. Estimation for the LGSSM [101] and three common approximate estimation methods for the Nssm [164] are described in more detail. There is an abundance of literature on the subject, see e.g. [79, 103, 159].

The general Bayesian solution to the PSsM is discussed in Section 5.1. An exact closed-form solution for the filtering and smoothing distributions of the LGSSM are presented in Sections 5.2.1 and 5.3, respectively. Approximate methods for the NSSM are presented in Sections 5.2.2-5.2.4. In Section 5.3 .3 a method proposed by the author for updating the smoothed posterior in a single pass with an observation at an arbitrary time is derived. In Section 5.4 an approximate method for filtering in the JMM is described and in Section 5.4.2 filtering for the model extension proposed in Section 3.6.2 is outlined. The latter method was first described by the author in [27]. Filtering for the extended target model in Section 3.7 is described in Section 5.5. The posterior distributions for the states and the timestamp in the UTM introduced in Section 3.8 are derived in Section 5.6 as well as four point estimators, which was published by the author in [182]. Estimation for the GPMM introduced in Section 3.9 is derived in Section 5.7 .

\subsection{Bayesian Solution}

The Bayesian solutions for filtering and smoothing are outlined in this section. For more details, see e.g. [159]. To simplify expressions, the notations $\mathcal{X}_{a: b} \triangleq$ $\left\{\mathbf{x}_{k}\right\}_{k=a}^{b}$ and $\mathcal{Y}_{a: b} \triangleq\left\{\mathbf{y}_{j}\right\}_{j \in\{a: b\} \cap \mathcal{J}}$ are defined with the shorthands $\mathcal{X} \triangleq \mathcal{X}_{0: K}$ and 
$\mathcal{Y} \triangleq \mathcal{Y}_{1: K}$. When applicable $\mathcal{X}_{a: b}$ and $\mathcal{Y}_{a: b}$ will be treated as a column vector with the vectors in the elements stacked vertically.

The first case considered is the filtering distribution. This is the posterior distribution $p\left(\mathbf{x}_{k} \mid \mathcal{Y}_{1: k}\right)$ of state $\mathbf{x}_{k}$ conditioned on all measurements $\mathcal{Y}_{1: k}$ up to and including time step $k$. This can be computed recursively using Bayes' theorem and the Chapman-Kolmogorov equations in a time update step

$$
\begin{aligned}
p\left(\mathbf{x}_{k} \mid \mathcal{Y}_{1: k-1}\right) & =\int p\left(\mathbf{x}_{k}, \mathbf{x}_{k-1} \mid \mathcal{Y}_{1: k-1}\right) d \mathbf{x}_{k-1} \\
& =\int p\left(\mathbf{x}_{k} \mid \mathbf{x}_{k-1}\right) p\left(\mathbf{x}_{k-1} \mid \mathcal{Y}_{1: k-1}\right) d \mathbf{x}_{k-1},
\end{aligned}
$$

where the integration is performed over the entire state space for $\mathbf{x}_{k-1}$, and a measurement update step, if a measurement is available,

$$
\begin{aligned}
p\left(\mathbf{x}_{k} \mid \mathcal{Y}_{1: k}\right) & =\frac{p\left(\mathbf{y}_{k} \mid \mathbf{x}_{k}, \mathcal{Y}_{1: k-1}\right) p\left(\mathbf{x}_{k} \mid \mathcal{Y}_{1: k-1}\right)}{p\left(\mathbf{y}_{k} \mid \mathcal{Y}_{1: k-1}\right)} \\
& \propto p\left(\mathbf{y}_{k} \mid \mathbf{x}_{k}\right) p\left(\mathbf{x}_{k} \mid \mathcal{Y}_{1: k-1}\right),
\end{aligned}
$$

where the Markov property is used in both steps. The symbol $\propto$ indicates equality up to a constant factor independent of $\mathbf{x}_{k}$ and the distribution can be recovered by normalization. Given the filtering distribution $p\left(\mathbf{x}_{k-1} \mid \mathcal{Y}_{1: k-1}\right)$ of the state for the previous time step and a PSSM as defined in (3.1), all distributions are available, and for the initial state a prior distribution $p\left(\mathbf{x}_{0}\right)$ is assumed.

The second case considered is the smoothing distribution. This is the posterior distribution $p\left(\mathbf{x}_{k} \mid \mathcal{Y}\right)$ of state $\mathbf{x}_{k}$ conditional on all the measurements $\mathcal{Y}$. This distribution is slightly more complicated to compute because in addition to computing the filtering distributions, called the forward recursive equations, requires a recursion backwards, [106], given by

$$
p\left(\mathbf{x}_{k} \mid \mathcal{Y}\right)=p\left(\mathbf{x}_{k} \mid \mathcal{Y}_{1: k}\right) \int \frac{p\left(\mathbf{x}_{k+1} \mid \mathbf{x}_{k}\right) p\left(\mathbf{x}_{k+1} \mid \mathcal{Y}\right)}{p\left(\mathbf{x}_{k+1} \mid \mathcal{Y}_{1: k}\right)} d \mathbf{x}_{k+1}
$$

Again, all distributions are available, either from the PSSM or the forward recursive equations. In general these recursions cannot be computed in closed form, with the LGSSM defined in (3.9) being a notable exception, but require numerical methods.

\subsection{Filter}

This section will present the Kalman filter (KF) for the LGSSM and two approximate solutions based on the KF for the NSSM. A stochastic method denoted the particle filter $(\mathrm{PF})$ is also briefly described.

\subsubsection{Kalman Filter}

As mentioned in Section 5.1, the filtering distribution for the LGSSM defined in (3.9) can be computed on closed form. The prior distribution of the initial state 
is Gaussian and it can be shown [159] that each step in the recursion results in a Gaussian distribution. The KF algorithm was first derived in [101].

Given the state posterior distribution at time step $k-1$ is $p\left(\mathbf{x}_{k-1} \mid \mathcal{Y}_{1: k-1}\right)=$ $\mathcal{N}\left(\mathbf{x}_{k-1} \mid \hat{\mathbf{x}}_{k-1 \mid k-1}, \mathbf{P}_{k-1 \mid k-1}\right)$, the time update in (5.1) reduces to

$$
\begin{aligned}
p\left(\mathbf{x}_{k} \mid \mathcal{Y}_{1: k-1}\right) & =\int p\left(\mathbf{x}_{k} \mid \mathbf{x}_{k-1}\right) p\left(\mathbf{x}_{k-1} \mid \mathcal{Y}_{1: k-1}\right) d \mathbf{x}_{k-1} \\
& =\mathcal{N}\left(\mathbf{x}_{k} \mid \mathbf{F}_{k} \hat{\mathbf{x}}_{k-1 \mid k-1}, \mathbf{F}_{k} \mathbf{P}_{k-1 \mid k-1} \mathbf{F}_{k}^{\top}+\mathbf{Q}_{k}\right) \\
& =\mathcal{N}\left(\mathbf{x}_{k} \mid \hat{\mathbf{x}}_{k \mid k-1}, \mathbf{P}_{k \mid k-1}\right),
\end{aligned}
$$

and the measurement update in (5.2) reduces to

$$
\begin{aligned}
p\left(\mathbf{x}_{k} \mid \mathcal{Y}_{1: k}\right) & =\frac{p\left(\mathbf{y}_{k} \mid \mathbf{x}_{k}, \mathcal{Y}_{1: k-1}\right) p\left(\mathbf{x}_{k} \mid \mathcal{Y}_{1: k-1}\right)}{p\left(\mathbf{y}_{k} \mid \mathcal{Y}_{1: k-1}\right)} \\
& =\mathcal{N}\left(\mathbf{x}_{k} \mid \hat{\mathbf{x}}_{k \mid k-1}+\mathbf{K}_{k}\left(\mathbf{y}_{k}-\mathbf{H}_{k} \hat{\mathbf{x}}_{k \mid k-1}\right), \mathbf{P}_{k \mid k-1}-\mathbf{K}_{k} \mathbf{H}_{k} \mathbf{P}_{k \mid k-1}\right) \\
& =\mathcal{N}\left(\mathbf{x}_{k} \mid \hat{\mathbf{x}}_{k \mid k}, \mathbf{P}_{k \mid k}\right),
\end{aligned}
$$

where

$$
\mathbf{K}_{k}=\mathbf{P}_{k \mid k-1} \mathbf{H}_{k}^{\top}\left(\mathbf{H}_{k} \mathbf{P}_{k \mid k-1} \mathbf{H}_{k}^{\top}+\mathbf{R}_{k}\right)^{-1} \text {. }
$$

See e.g. [159] for a derivation using joint and conditional distributions of Gaussians. Algorithm 5.1 conveniently summarizes (5.4) and (5.5), where the mean and covariance of the posterior distribution are propagated over time. The measurement update is repeated if there are multiple measurements at a time step and omitted if there are no measurements.

\section{Algorithm 5.1: Kalman Filter}

Input: $\overline{\mathbf{x}}_{0}, \mathbf{P}_{0}$ and $\mathbf{y}_{j}$ for $j \in \mathcal{J}$.

Initialization

$$
\begin{aligned}
& \hat{\mathbf{x}}_{0 \mid 0} \leftarrow \overline{\mathbf{x}}_{0} \\
& \mathbf{P}_{0 \mid 0} \leftarrow \mathbf{P}_{0}
\end{aligned}
$$

for $k=1, \ldots, K$ do

\section{Time Update}

$$
\begin{aligned}
& \hat{\mathbf{x}}_{k \mid k-1} \leftarrow \mathbf{F}_{k} \hat{\mathbf{x}}_{k-1 \mid k-1} \\
& \mathbf{P}_{k \mid k-1} \leftarrow \mathbf{F}_{k} \mathbf{P}_{k-1 \mid k-1} \mathbf{F}_{k}^{\top}+\mathbf{Q}_{k}
\end{aligned}
$$

Measurement Update

$$
\begin{aligned}
& \mathbf{S}_{k} \leftarrow \mathbf{H}_{k} \mathbf{P}_{k \mid k-1} \mathbf{H}_{k}^{\top}+\mathbf{R}_{k} \\
& \hat{\mathbf{y}}_{k} \leftarrow \mathbf{H}_{k} \hat{\mathbf{x}}_{k} \\
& \mathbf{K}_{k} \leftarrow \mathbf{P}_{k \mid k-1} \mathbf{H}_{k}^{\top} \mathbf{S}_{k}^{-1} \\
& \hat{\mathbf{x}}_{k \mid k} \leftarrow \hat{\mathbf{x}}_{k \mid k-1}+\mathbf{K}_{k}\left(\mathbf{y}_{k}-\hat{\mathbf{y}}_{k}\right) \\
& \mathbf{P}_{k \mid k} \leftarrow \mathbf{P}_{k \mid k-1}-\mathbf{K}_{k} \mathbf{H}_{k} \mathbf{P}_{k \mid k-1}
\end{aligned}
$$

end for

output: $\hat{\mathbf{x}}_{k \mid k}$ and $\mathbf{P}_{k \mid k}$ for $k \in \mathcal{K}$ 


\subsubsection{Extended Kalman Filter}

In the case of an NSSM, as defined in (3.3), the extended Kalman filter (EKF) [164] can be used to approximate the posterior distribution by its first two moments, the mean and the covariance. Affine approximations using the Jacobians of the transition and measurement functions and second moment approximations of the noise are used to propagate the mean and covariance over time. Algorithm 5.2 describes the steps involved in the EKF. The algorithm is no longer optimal and does not produce the exact Bayesian solution, but is common in practice due to its simplicity and decent performance in many applications [98]. However, there are several scenarios for which the algorithm does not perform well or even diverges, mainly where the linearizations or two-moment representations are poor approximations of the NSSM, for example when the noise is non-Gaussian or the distributions are multi-modal. The EKF is used for estimation in the dolphin application, however, considering the association uncertainty in the application the presentation is deferred to Application 6.3 in Chapter 6. The EKF is also used for estimation in the GPMм as described in Section 5.7.2.

\section{Algorithm 5.2: Extended Kalman Filter}

Input: $\overline{\mathbf{x}}_{0}, \mathbf{P}_{0}$ and $\mathbf{y}_{j}$ for $j \in \mathcal{J}$.

Initialization

$$
\begin{aligned}
& \hat{\mathbf{x}}_{0 \mid 0} \leftarrow \overline{\mathbf{x}}_{0} \\
& \mathbf{P}_{0 \mid 0} \leftarrow \mathbf{P}_{0}
\end{aligned}
$$

for $k=1, \ldots, K$ do

\section{Time Update}

$$
\begin{aligned}
& \mathbf{F}_{k}\left.\leftarrow \frac{\partial}{\partial \mathbf{x}} \mathbf{f}_{k}(\mathbf{x}, \mathbf{w})\right|_{\mathbf{x}=\hat{\mathbf{x}}_{k-1 \mid k-1}, \mathbf{w}=\mathbf{E}\left(\mathbf{w}_{k}\right)} \\
& \mathbf{L}_{k}\left.\leftarrow \frac{\partial}{\partial \mathbf{w}} \mathbf{f}_{k}(\mathbf{x}, \mathbf{w})\right|_{\mathbf{x}=\hat{\mathbf{x}}_{k-1 \mid k-1}, \mathbf{w}=\mathbf{E}\left(\mathbf{w}_{k}\right)} \\
& \mathbf{Q}_{k} \leftarrow \operatorname{Cov}\left(\mathbf{w}_{k}\right) \\
& \hat{\mathbf{x}}_{k \mid k-1} \leftarrow \mathbf{f}_{k}\left(\hat{\mathbf{x}}_{k-1 \mid k-1}, \mathbf{E}\left(\mathbf{w}_{k}\right)\right) \\
& \mathbf{P}_{k \mid k-1} \leftarrow \mathbf{F}_{k} \mathbf{P}_{k-1 \mid k-1} \mathbf{F}_{k}^{\top}+\mathbf{L}_{k} \mathbf{Q}_{k} \mathbf{L}_{k}^{\top} \\
& \text { Measurement Update }\left.\mathbf{H}_{k} \leftarrow \frac{\partial}{\partial \mathbf{x}} \mathbf{h}_{k}(\mathbf{x}, \mathbf{v})\right|_{\mathbf{x}=\hat{\mathbf{x}}_{k \mid k-1}, \mathbf{v}=\mathbf{E}\left(\mathbf{v}_{k}\right)} \\
&\left.\mathbf{M}_{k} \leftarrow \frac{\partial}{\partial \mathbf{v}} \mathbf{h}_{k}(\mathbf{x}, \mathbf{v})\right|_{\mathbf{x}=\hat{\mathbf{x}}_{k \mid k-1}, \mathbf{v}=\mathbf{E}\left(\mathbf{v}_{k}\right)} \\
&\left.\mathbf{R}_{k} \leftarrow \mathbf{C o v}_{(\mathbf{v}}\right) \\
& \mathbf{S}_{k} \leftarrow \mathbf{H}_{k} \mathbf{P}_{k \mid k-1} \mathbf{H}_{k}^{\top}+\mathbf{M}_{k} \mathbf{R}_{k} \mathbf{M}_{k}^{\top} \\
& \mathbf{K}_{k} \leftarrow \mathbf{P}_{k \mid k-1} \mathbf{H}_{k}^{\top} \mathbf{S}_{k}^{-1} \\
& \hat{\mathbf{x}}_{k \mid k} \leftarrow \hat{\mathbf{x}}_{k \mid k-1}+\mathbf{K}_{k}\left(\mathbf{y}_{k}-\mathbf{h}_{k}\left(\hat{\mathbf{x}}_{k \mid k-1}, \mathbf{E}\left(\mathbf{v}_{k}\right)\right)\right) \\
& \mathbf{P}_{k \mid k} \leftarrow \mathbf{P}_{k \mid k-1}-\mathbf{K}_{k} \mathbf{H}_{k} \mathbf{P}_{k \mid k-1}
\end{aligned}
$$

end for

Output: $\hat{\mathbf{x}}_{k \mid k}$ and $\mathbf{P}_{k \mid k}$ for $k \in \mathcal{K}$ 


\subsubsection{Iterated Extended Kalman Filter}

A common model in practice is the linear Gaussian motion model combined with a nonlinear measurement model with additive Gaussian noise. The iterated extended Kalman filter (IEKF), see [22], generally outperforms the EKF for this model at the expense of more computations. The basic idea is that the measurement update in the EKF can be seen as the first step in the Gauss-Newton method [138], and by applying the Gauss-Newton method until convergence the IEKF is obtained, resulting in a better approximation to the solution. The measurement update in Algorithm 5.2 is replaced by the measurement update in Algorithm 5.3. With $M=0$ the algorithm reduces to the EKF. The loop condition can also be replaced by a convergence criterion to improve performance. The IEKF is used in the filter bank for tracking in the bird application as described in Application 5.1 in Section 5.4.

\section{Algorithm 5.3: Iterated Extended Kalman Filter Measurement Update}

$$
\begin{aligned}
& \hat{\mathbf{x}}^{0}=\hat{\mathbf{x}}_{k \mid k-1} \\
& \text { for } i=0, \ldots, M \text { do } \\
& \left.\quad \mathbf{H}_{k}^{i} \leftarrow \frac{\partial}{\partial \mathbf{x}} \mathbf{h}_{k}(\mathbf{x}, \mathbf{v})\right|_{\mathbf{x}=\hat{\mathbf{x}}^{i}, \mathbf{v}=\mathbf{E} \mathbf{v}_{k}} \\
& \left.\quad \mathbf{M}_{k}^{i} \leftarrow \frac{\partial}{\partial \mathbf{v}} \mathbf{h}_{k}(\mathbf{x}, \mathbf{v})\right|_{\mathbf{x}=\hat{\mathbf{x}}^{i}, \mathbf{v}=\mathbf{E} \mathbf{v}_{k}}\left(\mathbf{S}_{k}^{i}\right. \\
& \quad \mathbf{S}_{k}^{i} \leftarrow \mathbf{H}_{k}^{i} \mathbf{P}_{k \mid k-1}\left(\mathbf{H}_{k}^{i}\right)^{\top}+\mathbf{M}_{k}^{i} \mathbf{R}_{k}\left(\mathbf{M}_{k}^{i}\right)^{\top} \\
& \quad \mathbf{K}_{k}^{i} \leftarrow \mathbf{P}_{k \mid k-1}\left(\mathbf{H}_{k}^{i}\right)^{\top}\left(\mathbf{S}_{k}^{i}\right)^{-1} \\
& \quad \hat{\mathbf{x}}^{i+1} \leftarrow \hat{\mathbf{x}}_{k \mid k-1}+\mathbf{K}_{k}^{i}\left(\mathbf{y}_{k}-\mathbf{h}_{k}\left(\hat{\mathbf{x}}^{i}, \overline{\mathbf{v}}_{k}\right)-\mathbf{H}_{k}^{i}\left(\hat{\mathbf{x}}_{k \mid k-1}-\hat{\mathbf{x}}^{i}\right)\right) \\
& \text { end for } \\
& \hat{\mathbf{x}}_{k \mid k} \leftarrow \hat{\mathbf{x}}^{M+1} \\
& \mathbf{P}_{k \mid k} \leftarrow \mathbf{P}_{k \mid k-1}-\mathbf{K}_{k}^{M} \mathbf{H}_{k}^{M} \mathbf{P}_{k \mid k-1}
\end{aligned}
$$

\subsubsection{Particle Filter}

In the case of an NSSM another approach can also be employed where the distributions are represented by weighted samples denoted sequential Monte Carlo or a $\mathrm{PF}[76,82]$. The main idea is that a distribution is approximated by

$$
p(\mathbf{x}) \approx \sum_{i=1}^{N} w^{i} \delta_{\mathbf{x}^{i}}(\mathbf{x}),
$$

where $N$ is the number of samples, denoted particles, $\mathbf{x}^{i}$ is a particle with weight $w^{i}$ and $\delta_{\mathbf{a}}(\mathbf{b})$ is the Dirac delta function. Using this approximation of the distributions when performing the recursion in (5.1) and (5.2), the Bayes solution to the filtering problem is approximated. Implementations of this filter often suffer from practical issues, such as particle depletion, so additional steps are required for efficient solutions. A simple implementation is presented in Algorithm 5.4, where the $\sim$ denotes drawing a sample from a distribution. To avoid particle 
depletion, where all but a few particles have negligible weights, the particles are resampled using importance sampling [82]. When the effective number of particles $N_{\text {eff }}=\left(\sum_{i=1}^{N}\left(w^{i}\right)^{2}\right)^{-1}$ decreases below a threshold $N_{t h}$, the particles are resampled with replacement with the probabilities given by their weights. The PF is used in the sea ice tracking application described in Application 6.4.

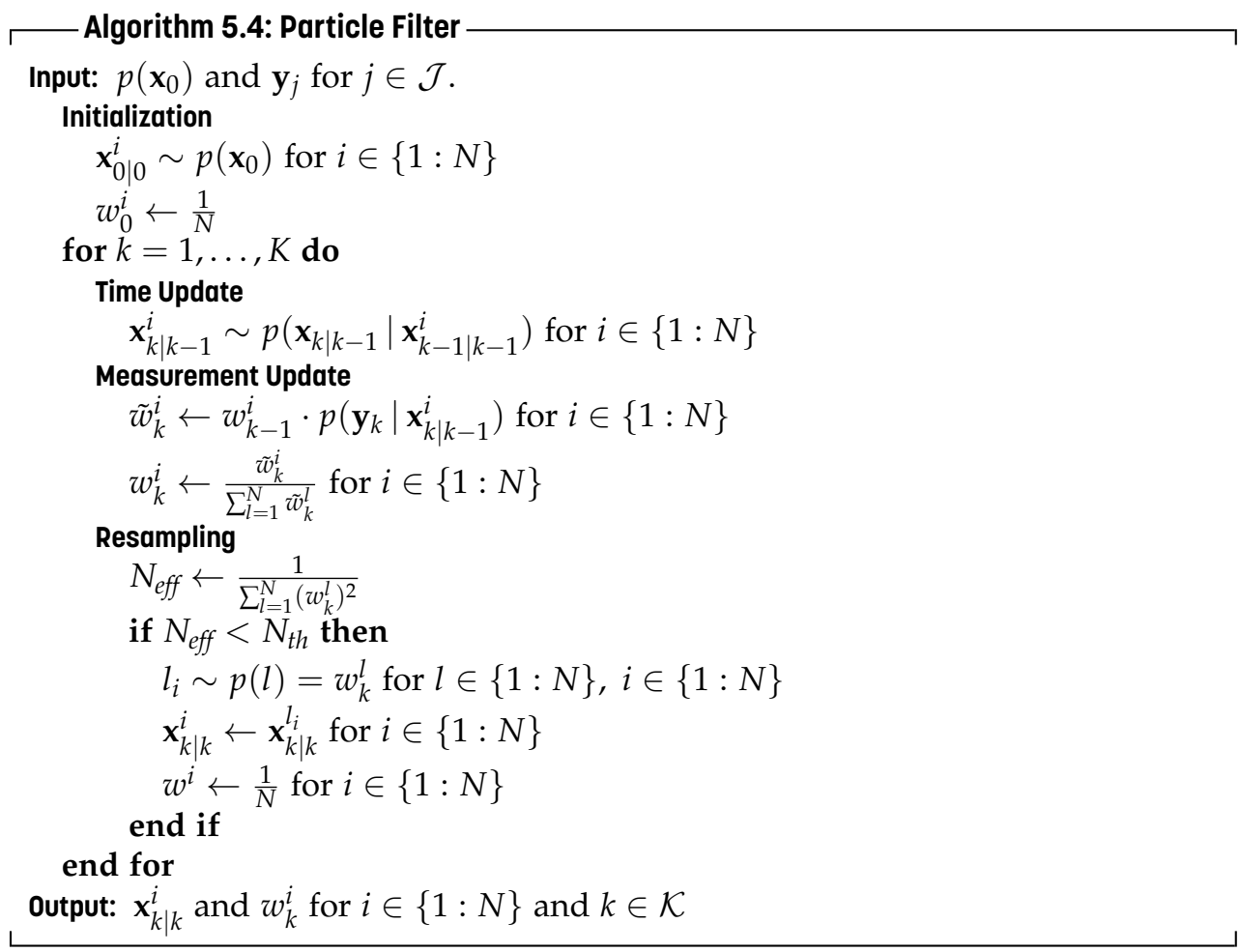

\subsection{Smoother}

A method for computing the smoothing posterior distribution for the full state vector in the LGSSM is described [81] and a common two-pass recursive algorithm [159] is presented in Section 5.3.1. In Sections 5.3.2-5.3.3 an algorithm is proposed by the author for updating the smoothing posterior distribution with an observation at an arbitrary timestamp in a single pass.

\subsubsection{Linear Gaussian Smoothing}

When all measurements are available the smoothing distribution $p\left(\mathbf{x}_{k} \mid \mathcal{Y}\right)$ can be obtained as in (5.3). For an LGSSM, the posterior distribution is Gaussian and its parameters can be computed on closed form [37]. Any distribution $p\left(\mathbf{x}_{k} \mid \mathcal{Y}_{1: k+a}\right)$ 
for $a>0$ is commonly considered a smoothing distribution, however, only the smoothing distribution, $p(\mathcal{X} \mid \mathcal{Y})$, for an entire batch of measurements is considered in this section.

A straightforward solution is obtained by rearranging the LGSSM in (3.9) into a system of equations,

$$
\begin{aligned}
& \mathbf{0}=\mathbf{x}_{k}-\mathbf{F}_{k} \mathbf{x}_{k-1}+\mathbf{w}_{k}, \quad \mathbf{w}_{k} \sim \mathcal{N}\left(\mathbf{0}, \mathbf{Q}_{k}\right), \quad k \in \mathcal{K}, \\
& \mathbf{y}_{j}=\mathbf{H}_{j} \mathbf{x}_{k_{j}}+\mathbf{v}_{j}, \quad \mathbf{v}_{j} \sim \mathcal{N}\left(\mathbf{0}, \mathbf{R}_{j}\right), \quad j \in \mathcal{J} \text {, } \\
& \overline{\mathbf{x}}_{0}=\mathbf{x}_{0}+\mathbf{e}_{\mathbf{x}_{0}}, \quad \mathbf{e}_{\mathbf{x}_{0}} \sim \mathcal{N}\left(\mathbf{0}, \mathbf{P}_{0}\right),
\end{aligned}
$$

and rewriting each equation on the form

$$
\overline{\mathbf{y}}_{p}=\overline{\mathbf{H}}_{p} \mathcal{X}+\overline{\mathbf{E}}_{p}, \quad p \in \mathcal{P}=\{1: K+J+1\},
$$

where $\operatorname{cov}\left(\overline{\mathbf{E}}_{p}\right)=\overline{\mathbf{R}}_{p}$. For affine models, $\overline{\mathbf{y}}_{p}$ is modified to account for the constant term. The posterior distribution of the full state vector is then computed as [144]

$$
\begin{aligned}
p(\mathcal{X} \mid \mathcal{Y}) & \propto p(\mathcal{Y} \mid \mathcal{X}) p(\mathcal{X})=\prod_{j \in \mathcal{J}} p\left(\mathbf{y}_{j} \mid \mathbf{x}_{k_{j}}\right) \prod_{k \in \mathcal{K}} p\left(\mathbf{x}_{k} \mid \mathbf{x}_{k-1}\right) p\left(\mathbf{x}_{0}\right) \\
& =\prod_{j \in \mathcal{J}} \mathcal{N}\left(\mathbf{y}_{j} \mid \mathbf{H}_{j} \mathbf{x}_{k_{j}}, \mathbf{R}_{j}\right) \prod_{k \in \mathcal{K}} \mathcal{N}\left(\mathbf{x}_{k} \mid \mathbf{F}_{k} \mathbf{x}_{k-1}, \mathbf{Q}_{k}\right) \mathcal{N}\left(\mathbf{x}_{0} \mid \overline{\mathbf{x}}_{0}, \mathbf{P}_{0}\right) \\
& =\prod_{p \in \mathcal{P}} \mathcal{N}\left(\overline{\mathbf{y}}_{p} \mid \overline{\mathbf{H}}_{p} \mathcal{X}, \overline{\mathbf{R}}_{p}\right) \propto \exp \left(-\frac{1}{2} \sum_{p \in \mathcal{P}}\left\|\overline{\mathbf{y}}_{p}-\overline{\mathbf{H}}_{p} \mathcal{X}\right\|_{\overline{\mathbf{R}}_{p}}^{2}\right) \\
& \propto \exp \left(-\frac{1}{2}\left\|\mathcal{X}-\mathcal{I}^{-1} \iota\right\|_{\mathcal{I}^{-1}}^{2}\right) \\
& \propto \mathcal{N}\left(\mathcal{X} \mid \mathcal{I}^{-1} \iota, \mathcal{I}^{-1}\right)=\mathcal{N}(\mathcal{X} \mid \hat{\mathcal{X}}, \mathbf{P}),
\end{aligned}
$$

using Bayes' theorem, the independence and Markov assumptions and matrix completion of squares, where

$$
\begin{aligned}
\mathcal{I} & =\sum_{p \in \mathcal{P}} \overline{\mathbf{H}}_{p}^{\top} \overline{\mathbf{R}}_{p}^{-1} \overline{\mathbf{H}}_{p}, \\
\boldsymbol{\iota} & =\sum_{p \in \mathcal{P}} \overline{\mathbf{H}}_{p}^{\top} \overline{\mathbf{R}}_{p}^{-1} \overline{\mathbf{y}}_{p} .
\end{aligned}
$$

The matrix $\mathcal{I}$ is often denoted the information matrix and $\iota$ the information state [81]. Note that each equation in the model in (3.13) contributes one term to the information matrix and the information state, respectively.

An efficient recursive solution to the posterior distribution of the marginal state vector can be obtained using the Rauch-Tung-Striebel smoother (RTSS) [152]. The RTSS is executed in a forward and a backward recursion, where the former is equivalent to the KF. The mean vectors $\hat{\mathbf{x}}_{k \mid k-1}$ and $\hat{\mathbf{x}}_{k \mid k}$ and covariances $\mathbf{P}_{k \mid k-1}$ and $\mathbf{P}_{k \mid k}, k \in \mathcal{K}$, in the prediction distributions (5.4) and the filtering distributions (5.5), respectively, are stored. The backward recursion in (5.3) is computed as

$$
\hat{\mathbf{x}}_{k-1 \mid K}=\hat{\mathbf{x}}_{k-1 \mid k-1}+\mathbf{P}_{k-1 \mid k-1} \mathbf{F}_{k}^{\top} \mathbf{P}_{k \mid k-1}^{-1}\left(\hat{\mathbf{x}}_{k \mid K}-\hat{\mathbf{x}}_{k \mid k-1}\right),
$$




$$
\mathbf{P}_{k-1 \mid K}=\mathbf{P}_{k-1 \mid k-1}+\mathbf{P}_{k-1 \mid k-1} \mathbf{F}_{k}^{\top} \mathbf{P}_{k \mid k-1}^{-1}\left(\mathbf{P}_{k \mid K}-\mathbf{P}_{k \mid k-1}\right) \mathbf{P}_{k \mid k-1}^{-1} \mathbf{F}_{k} \mathbf{P}_{k-1 \mid k-1} .
$$

To completely represent the posterior distribution of the full state vector, $p(\mathcal{X} \mid \mathcal{Y})$, the cross-covariances between consecutive states are required [127]. A slight modification of the backward recursion in the RTSS achieves this by computing [159]

$$
\begin{aligned}
\mathbf{P}_{k-1, k \mid K} & =\mathbf{P}_{k-1 \mid k-1} \mathbf{F}_{k}^{\top}\left(\mathbf{F}_{k} \mathbf{P}_{k-1 \mid k-1} \mathbf{F}_{k}^{\top}+\mathbf{Q}_{k}\right)^{-1} \mathbf{P}_{k \mid K} \\
& =\mathbf{P}_{k-1 \mid k-1} \mathbf{F}_{k}^{\top} \mathbf{P}_{k \mid k-1}^{-1} \mathbf{P}_{k} .
\end{aligned}
$$

The modified RTss is outlined in Algorithm 5.5, and it results in the marginalized posterior distribution given by

$$
p\left(\mathbf{x}_{k} \mid \mathcal{Y}\right)=\mathcal{N}\left(\mathbf{x}_{k} \mid \hat{\mathbf{x}}_{k \mid K}, \mathbf{P}_{k \mid K}\right),
$$

and the cross-covariances $\operatorname{Cov}\left(\mathbf{x}_{k-1}, \mathbf{x}_{k} \mid \mathcal{Y}\right)$ between consecutive states for $k \in \mathcal{K}$.

The RTSS can be also be applied to NSSMs by replacing the forward recursion with an EKF as in [159], referred to as the extended RTSS. To further improve the estimate, the extended RTSS can be applied iteratively until convergence by linearizing around the current estimate in the forward recursion of each new iteration [21]. As for the EKF, the posterior distribution is approximate and no convergence guarantees exist.

\section{Algorithm 5.5: Rauch-Tung-Striebel Smoother}

Input: $\overline{\mathbf{x}}_{0}, \mathbf{P}_{0}$ and $\mathbf{y}_{j}$ for $j \in \mathcal{J}$.

Compute $\hat{\mathbf{x}}_{k \mid k-1}, \hat{\mathbf{x}}_{k \mid k}, \mathbf{P}_{k \mid k-1}$ and $\mathbf{P}_{k \mid k}$ for $k \in \mathcal{K}$ using Algorithm 5.2.

for $k=K, \ldots, 1$ do

$$
\begin{aligned}
& \quad \hat{\mathbf{x}}_{k-1 \mid K} \leftarrow \hat{\mathbf{x}}_{k-1 \mid k-1}+\mathbf{P}_{k-1 \mid k-1} \mathbf{F}_{k}^{\top} \mathbf{P}_{k \mid k-1}^{-1}\left(\hat{\mathbf{x}}_{k \mid K}-\hat{\mathbf{x}}_{k \mid k-1}\right) \\
& \qquad \mathbf{P}_{k-1 \mid K} \leftarrow \mathbf{P}_{k-1 \mid k-1}+\mathbf{P}_{k-1 \mid k-1} \mathbf{F}_{k}^{\top} \mathbf{P}_{k \mid k-1}^{-1}\left(\mathbf{P}_{k \mid K}-\mathbf{P}_{k \mid k-1}\right) \mathbf{P}_{k \mid k-1}^{-1} \mathbf{F}_{k} \mathbf{P}_{k-1 \mid k-1} \\
& \quad \mathbf{P}_{k-1, k \mid K} \leftarrow \mathbf{P}_{k-1 \mid k-1} \mathbf{F}_{k}^{\top} \mathbf{P}_{k \mid k-1}^{-1} \mathbf{P}_{k \mid K} \\
& \text { end for }
\end{aligned}
$$

Output: $\hat{\mathbf{x}}_{k \mid K}$ and $\mathbf{P}_{k \mid K}$ for $k \in 0 \cup \mathcal{K}$ and $\mathbf{P}_{k-1, k \mid K}$ for $k \in \mathcal{K}$

The RTSS is used for estimation in the uncertain time scenarios, however, considering the uncertainty in time the presentation is deferred to Section 5.6.

\subsubsection{State Injection}

Given the posterior smoothing distribution of the states $\mathcal{X}$ at the time instants in $\mathcal{T}_{x}$ for the continuous-discrete LGSSM in (3.13), it is possible to obtain the joint posterior distribution of the states $\mathcal{X}$ and the state $\mathbf{x}\left(t_{\tau}\right)$ for an arbitrary time $t_{\tau}$, where $t_{k-1}<t_{\tau}<t_{k}$. This is straightforward to compute using the RTSS in Section 5.3.1 for the new set of time instants with a large amount of redundancy. However, a more efficient method is proposed using the results in Proposition 5.1. See the illustration in Figure 5.1. 


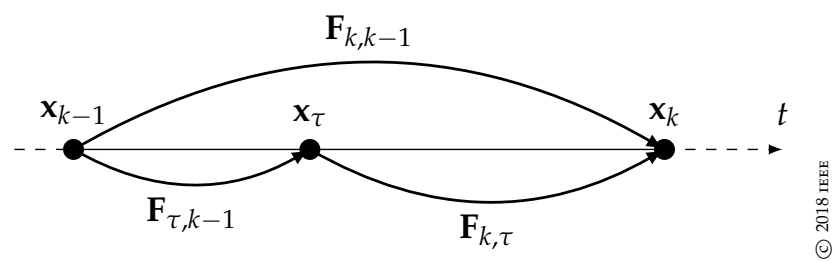

Figure 5.1: Illustrates the injection of the state at time $t_{\tau}$ given the joint posterior distribution of the states at times $t_{k-1}$ and $t_{k}$. The modified equations need to satisfy the constraints in (3.15). Published in [183].

Proposition 5.1: Given the posterior distribution $p(\mathcal{X} \mid \mathcal{Y})=\mathcal{N}(\mathcal{X} \mid \hat{\mathcal{X}}, \mathbf{P})$ for the model in (3.13), consider that the model is modified by substituting the equation

$$
\mathbf{x}_{k}=\mathbf{F}_{k, k-1} \mathbf{x}_{k-1}+\mathbf{w}_{k, k-1},
$$

by the two equations

$$
\begin{aligned}
& \mathbf{x}_{\tau}=\mathbf{F}_{\tau, k-1} \mathbf{x}_{k-1}+\mathbf{w}_{\tau, k-1}, \\
& \mathbf{x}_{k}=\mathbf{F}_{k, \tau} \mathbf{x}_{\tau}+\mathbf{w}_{k, \tau},
\end{aligned}
$$

where $t_{k-1}<t_{\tau}<t_{k}$ and the transition and covariance matrices involved satisfy the conditions in (3.15). The posterior distribution of the modified model is then given by

$$
p\left(\left[\begin{array}{c}
\mathcal{X}_{m} \\
\mathbf{x}_{\tau}
\end{array}\right] \mid \mathcal{Y}\right)=\mathcal{N}\left(\left[\begin{array}{c}
\mathcal{X}_{m} \\
\mathbf{x}_{\tau}
\end{array}\right] \mid\left[\begin{array}{c}
\hat{\mathcal{X}} \\
\mathbf{K} \hat{\mathcal{X}}
\end{array}\right],\left[\begin{array}{cc}
\mathbf{P} & \mathbf{P K}^{\top} \\
\mathbf{K} \mathbf{P} & \mathbf{D}^{-1}+\mathbf{K P K}^{\top}
\end{array}\right]\right),
$$

where $\mathcal{X}_{m}$ denotes all states in the modified model excluding $\mathbf{x}_{\tau}$ and

$$
\begin{aligned}
\mathbf{I}_{k} \mathcal{X} & =\left(\begin{array}{c}
\mathbf{x}_{k-1} \\
\mathbf{x}_{k}
\end{array}\right), \\
\mathbf{D} & =\mathbf{Q}_{\tau, k-1}^{-1}+\mathbf{F}_{k, \tau}^{\top} \mathbf{Q}_{k, \tau}^{-1} \mathbf{F}_{k, \tau}, \\
\mathbf{C} & =\left(\begin{array}{ll}
\mathbf{Q}_{\tau, k-1}^{-1} \mathbf{F}_{\tau, k-1} & \mathbf{F}_{k, \tau}^{\top} \mathbf{Q}_{k, \tau}^{-1}
\end{array}\right), \\
\mathbf{K} & =\mathbf{D}^{-1} \mathbf{C I}_{k} .
\end{aligned}
$$

Proof: The proof is presented in Appendix A.2.

The proposition implies that the states $\mathcal{X}$ are unaffected by the modification and that only the cross-covariances between consecutive states are required for state injection. Removing a state is achieved by marginalizing out the state. For a Gaussian distribution this essentially comprises removing the state from the mean vector and covariance matrix.

\subsubsection{Update of Posterior Distribution}

Given the smoothed posterior distribution of the states $\mathcal{X}$ at the time instants in $\mathcal{T}_{x}$, one wants to condition the distribution on an additional measurement $\mathbf{z}$ at a 
time $t_{\tau} \in \mathcal{T}_{x}$. Instead of recomputing the posterior distribution, a more efficient method is proposed for the LGSSM using the results in Proposition 5.2.

Proposition 5.2: Given the posterior distribution $p(\mathcal{X} \mid \mathcal{Y})=\mathcal{N}(\mathcal{X} \mid \hat{\mathcal{X}}, \mathbf{P})$ for the model in (3.13), represented and uniquely determined by

$$
\begin{aligned}
\hat{\mathbf{x}}_{k} & =\mathrm{E}\left(\mathbf{x}\left(t_{k}\right)\right), & & k \in 0 \cup \mathcal{K}, \\
\mathbf{P}_{k} & =\operatorname{Cov}\left(\mathbf{x}\left(t_{k}\right)\right), & & k \in 0 \cup \mathcal{K}, \\
\mathbf{P}_{k-1, k} & =\operatorname{Cov}\left(\mathbf{x}\left(t_{k-1}\right), \mathbf{x}\left(t_{k}\right)\right), & & k \in \mathcal{K},
\end{aligned}
$$

consider that the model is modified by adding the equation

$$
\mathbf{z}=\mathbf{H x}\left(t_{\tau}\right)+\mathbf{e}, \quad \mathbf{e} \sim \mathcal{N}(\mathbf{0}, \mathbf{R}),
$$

where $\tau \in 0 \cup \mathcal{K}$. The posterior distribution $p(\mathcal{X} \mid \mathcal{Y}, \mathbf{z})=\mathcal{N}\left(\mathcal{X} \mid \hat{\mathcal{X}}^{+}, \mathbf{P}^{+}\right)$of the modified model is then given by

$$
\begin{aligned}
\hat{\mathbf{x}}_{\tau}^{+} & =\hat{\mathbf{x}}_{\tau}+\mathbf{P}_{\tau} \mathbf{H}^{\top} \mathbf{S}_{\tau}^{-1}\left(\mathbf{z}-\mathbf{H} \hat{\mathbf{x}}_{\tau}\right), \\
\mathbf{P}_{\tau}^{+} & =\mathbf{P}_{\tau}-\mathbf{P}_{\tau} \mathbf{H}^{\top} \mathbf{S}_{\tau}^{-1} \mathbf{H} \mathbf{P}_{\tau},
\end{aligned}
$$

for the instant of time $t_{\tau}$, where $\mathbf{S}_{\tau}=\mathbf{H P}_{\tau} \mathbf{H}^{\top}+\mathbf{R}$, and recursively computed by

$$
\begin{aligned}
\hat{\mathbf{x}}_{k}^{+} & =\hat{\mathbf{x}}_{k}+\mathbf{K}_{k}^{b}\left(\hat{\mathbf{x}}_{k+1}^{+}-\hat{\mathbf{x}}_{k+1}\right), \\
\mathbf{P}_{k, k+1}^{+} & =\mathbf{P}_{k, k+1}+\mathbf{K}_{k}^{b}\left(\mathbf{P}_{k+1}^{+}-\mathbf{P}_{k+1}\right), \\
\mathbf{P}_{k}^{+} & =\mathbf{P}_{k}+\left(\mathbf{P}_{k, k+1}^{+}-\mathbf{P}_{k, k+1}\right)\left(\mathbf{K}_{k}^{b}\right)^{\top},
\end{aligned}
$$

for $k<\tau$, where $\mathbf{K}_{k}^{b}=\mathbf{P}_{k, k+1} \mathbf{P}_{k+1}^{-1}$, and by

$$
\begin{aligned}
\hat{\mathbf{x}}_{k}^{+} & =\hat{\mathbf{x}}_{k}+\mathbf{K}_{k}^{f}\left(\hat{\mathbf{x}}_{k-1}^{+}-\hat{\mathbf{x}}_{k-1}\right), \\
\mathbf{P}_{k-1, k}^{+} & =\mathbf{P}_{k-1, k}+\left(\mathbf{P}_{k-1}^{+}-\mathbf{P}_{k-1}\right)\left(\mathbf{K}_{k}^{f}\right)^{\top}, \\
\mathbf{P}_{k}^{+} & =\mathbf{P}_{k}+\mathbf{K}_{k}^{f}\left(\mathbf{P}_{k-1, k}^{+}-\mathbf{P}_{k-1, k}\right) .
\end{aligned}
$$

for $k>\tau$, where $\mathbf{K}_{k}^{f}=\mathbf{P}_{k-1, k}^{\top} \mathbf{P}_{k-1}^{-1}$.

Proof: The proof is presented in Appendix A.3.

Combining (5.21)-(5.23) results in a method for efficiently updating a smoothed posterior distribution with an additional measurement, described in Algorithm 5.6 and first published in [180]. A previously added measurement can be removed by subtracting its contribution, which artificially can be done using the algorithm together with a negative measurement noise covariance matrix.

The main advantage of this approach is that only a single pass is required to update the smoothed posterior distribution compared to two passes required for the RTSS. In addition, the approach allows for further reduction in computation cost by terminating the forward and backward updates early when the contributions from the updates in (5.22) and (5.23) are negligible. 
One criterion for termination is to require a minimum Kullback-Leibler divergence [110] between the previous distribution and the updated distribution. For the two Gaussian distributions $\mathcal{N}\left(\hat{\mathbf{x}}_{k}, \mathbf{P}_{k}\right)$ and $\mathcal{N}\left(\hat{\mathbf{x}}_{k}^{+}, \mathbf{P}_{k}^{+}\right)$, the divergence is given by

$$
D_{K L}=\frac{1}{2}\left(D_{M}-\operatorname{tr}\left(\mathbf{I}-\mathbf{P}_{k}^{-1} \mathbf{P}_{k}^{+}\right)-\log \left(\left|\mathbf{P}_{k}^{-1} \mathbf{P}_{k}^{+}\right|\right)\right)
$$

where

$$
D_{M}=\left(\hat{\mathbf{x}}_{k}^{+}-\hat{\mathbf{x}}\right)^{\top}\left(\mathbf{P}_{k}^{+}\right)^{-1}\left(\hat{\mathbf{x}}_{k}^{+}-\hat{\mathbf{x}}\right),
$$

represents the squared Mahalanobis distance.

\subsection{Jump Markov Model Filter}

A method for computing the filtering distribution of a JMM, denoted a filter bank, is presented in this section, described in e.g. [81]. A combinatoric explosion occurs when considering all possible mode switches, so pruning of unlikely mode sequences is required for tractability. The filter bank for the JMM is described in Section 5.4.1. The modifications required for estimation in the extension of the JMM proposed in Section 3.6.2 is described in Section 5.4.2, which was first published by the author in [27].

\subsubsection{General Model}

Given the model in (3.45), one is interested in the posterior distribution of the states $\mathbf{x}_{k}$ and modes $\delta_{k}, k \in \mathcal{K}$. For a given mode sequence the model reduces to an AWGNSSM and the filtering and smoothing posterior distributions can be approximately computed using, e.g., Algorithms 5.2 and 5.5, respectively.

When the mode sequence $\Delta=\left\{\delta_{k}\right\}_{k \in \mathcal{K}}$ is unknown the posterior is a mixture of $|\mathcal{S}|^{N}$ distributions. By indexing each unique sequence $\Delta_{1: k}^{i}=\left\{\delta_{j}^{i}\right\}_{j=1}^{k}, i \in\{1$ : $\left.|\mathcal{S}|^{k}\right\}$, the posterior distribution can be computed as

$$
p\left(\mathbf{x}_{k} \mid \mathcal{Y}_{1: k}\right)=\sum_{i=1}^{|\mathcal{S}|^{k}} p\left(\mathbf{x}_{k}, \Delta_{1: k}^{i} \mid \mathcal{Y}_{1: k}\right)=\sum_{i=1}^{|\mathcal{S}|^{k}} p\left(\Delta_{1: k}^{i} \mid \mathcal{Y}_{1: k}\right) p\left(\mathbf{x}_{k} \mid \Delta_{1: k}^{i}, \mathcal{Y}_{1: k}\right)
$$

The weight for each distribution in the mixture is computed recursively using

$$
\begin{aligned}
w_{k}^{i} & \triangleq p\left(\Delta_{1: k}^{i} \mid \mathcal{Y}_{1: k}\right)=\frac{p\left(\Delta_{1: k}^{i}\right) p\left(\mathcal{Y}_{1: k} \mid \Delta_{1: k}^{i}\right)}{p\left(\mathcal{Y}_{1: k}\right)} \\
& =\frac{p\left(\Delta_{1: k-1}^{i}\right) p\left(\mathcal{Y}_{1: k-1} \mid \Delta_{1: k}^{i}\right)}{p\left(\mathcal{Y}_{1: k-1}\right)} \cdot p\left(\delta_{k}^{i} \mid \delta_{k-1}^{i}\right) \cdot \frac{p\left(\mathbf{y}_{k} \mid \mathcal{Y}_{1: k-1}, \Delta_{1: k}^{i}\right)}{p\left(\mathbf{y}_{k} \mid \mathcal{Y}_{1: k-1}\right)} \\
& =w_{k-1}^{i} \Pi_{k}^{\delta_{k}^{i}, \delta_{k-1}^{i}} \frac{\int_{\mathbf{x}_{k}} p\left(\mathbf{y}_{k} \mid \mathbf{x}_{k}, \delta_{k}^{i}\right) p\left(\mathbf{x}_{k} \mid \mathcal{Y}_{1: k-1}, \Delta_{1: k}^{i}\right) d \mathbf{x}_{k}}{p\left(\mathbf{y}_{k} \mid \mathcal{Y}_{1: k-1}\right)}
\end{aligned}
$$




\section{Algorithm 5.6: Update of Smoothed Posterior Distribution}

Input: $\mathbf{z}, \tau, \hat{\mathbf{x}}_{k}$ and $\mathbf{P}_{k}$ for $k \in 0 \cup \mathcal{K}$, and $\mathbf{P}_{k, k+1}$ for $k \in \mathcal{K}$, Initialization

$$
\begin{aligned}
& \mathbf{K} \leftarrow \mathbf{P}_{\tau} \mathbf{H}^{\top}\left(\mathbf{H} \mathbf{P}_{\tau} \mathbf{H}^{\top}+\mathbf{R}\right)^{-1} \\
& \mathbf{D}_{\tau} \leftarrow-\mathbf{K} \mathbf{H} \mathbf{P}_{\tau} \\
& \mathbf{P}_{\tau}^{+} \leftarrow \mathbf{P}_{\tau}+\mathbf{D}_{\tau} \\
& \mathbf{d}_{\tau} \leftarrow \mathbf{K}\left(\mathbf{z}-\mathbf{H} \hat{\mathbf{x}}_{\tau}\right) \\
& \hat{\mathbf{x}}_{\tau}^{+} \leftarrow \hat{\mathbf{x}}_{\tau}+\mathbf{d}_{\tau}
\end{aligned}
$$

Backward Update

$$
\begin{aligned}
& \mathbf{D} \leftarrow \mathbf{D}_{\tau} \\
& \mathbf{d} \leftarrow \mathbf{d}_{\tau}
\end{aligned}
$$$$
\text { for } k=\tau-1 \text { to } 0 \text { do }
$$$$
\mathbf{K} \leftarrow \mathbf{P}_{k, k+1} \mathbf{P}_{k+1}^{-1}
$$$$
\mathbf{D} \leftarrow \mathbf{K D}
$$$$
\mathbf{P}_{k, k+1}^{+} \leftarrow \mathbf{P}_{k, k+1}+\mathbf{D}
$$$$
\mathbf{D} \leftarrow \mathbf{D K}^{\top}
$$$$
\mathbf{P}_{k}^{+} \leftarrow \mathbf{P}_{k}+\mathbf{D}
$$$$
\mathbf{d} \leftarrow \mathbf{K d}
$$$$
\hat{\mathbf{x}}_{k}^{+} \leftarrow \hat{\mathbf{x}}_{k}+\mathbf{d}
$$

end for

\section{Forward Update}

$$
\begin{aligned}
& \mathbf{D} \leftarrow \mathbf{D}_{\tau} \\
& \mathbf{d} \leftarrow \mathbf{d}_{\tau} \\
& \text { for } k=\tau+1 \text { to } K \text { do } \\
& \quad \mathbf{K} \leftarrow \mathbf{P}_{k-1, k}^{\top} \mathbf{P}_{k-1}^{-1} \\
& \mathbf{D} \leftarrow \mathbf{D} \mathbf{K}^{\top} \\
& \mathbf{P}_{k-1, k}^{+} \leftarrow \mathbf{P}_{k-1, k}+\mathbf{D} \\
& \mathbf{D} \leftarrow \mathbf{K D} \\
& \mathbf{P}_{k}^{+} \leftarrow \mathbf{P}_{k}+\mathbf{D} \\
& \quad \mathbf{d} \leftarrow \mathbf{K d} \\
& \hat{\mathbf{x}}_{k}^{+} \leftarrow \hat{\mathbf{x}}_{k}+\mathbf{d} \\
& \text { end for }
\end{aligned}
$$

Output: $\hat{\mathbf{x}}_{k}^{+}$and $\mathbf{P}_{k}^{+}$for $k \in 0 \cup \mathcal{K}$, and $\mathbf{P}_{k, k+1}^{+}$for $k \in \mathcal{K}$ 
where the Markov property of both the mode and the SSM is used. For the LGSSM, this reduces to

$$
\begin{aligned}
w_{k}^{i} & \propto w_{k-1}^{i} \boldsymbol{\Pi}_{k}^{\delta_{k}^{i} \delta_{k-1}^{i}} \mathcal{N}\left(\mathbf{y}_{k} \mid \mathbf{H}_{k}^{\delta_{k}^{i}} \hat{\mathbf{x}}_{k \mid k-1}^{\Delta_{1: k}^{i}}, \mathbf{H}_{k}^{\delta_{k}^{i}} \mathbf{P}_{k \mid k-1}^{\Delta_{1: k}^{i}} \mathbf{H}_{k}^{\delta_{k}^{i}}+\mathbf{R}_{k}\left(\delta_{k}^{i}\right)\right) \\
& =w_{k-1}^{i} \boldsymbol{\Pi}_{k}^{\delta_{k}^{i}, \delta_{k-1}^{i}} \mathcal{N}\left(\mathbf{y}_{k} \mid \hat{\mathbf{y}}_{k}^{i}, \mathbf{S}_{k}^{i}\right),
\end{aligned}
$$

where $\mathbf{h}_{k}\left(\mathbf{x}_{k}, \delta_{k}\right)=\mathbf{H}_{k}^{\delta_{k}} \mathbf{x}_{k}$, and $p\left(\mathbf{x}_{k} \mid \mathcal{Y}_{1: k-1}, \Delta_{1: k}\right)=\mathcal{N}\left(\mathbf{x}_{k} \mid \hat{\mathbf{x}}_{k \mid k-1}^{\Delta_{1: k}}, \mathbf{P}_{k \mid k-1}^{\Delta_{1: k}}\right)$ is obtained using the $\mathrm{KF}$ in Algorithm 5.2 and the filtering posterior distribution results in a mixture of Gaussians.

Due to the number of distributions in the mixture, it is intractable to compute the complete posterior distribution. Often in practice, only the distributions with the highest weights are computed in real-time applications, or, when all measurements are available, the EM algorithm [19, 53, 116] or Markov Chain Monte Carlo methods [23] can be used to approximate the posterior distribution.

A common method for real-time applications is to view the growing number of unique sequences as a tree and prune or merge low-probability branches [32]. A method that keeps the $M$ most likely branches by pruning is presented in Algorithm 5.7. The main idea is that old high-probability branches are extended with all possible modes for the current time step in a sequence-splitting step. A time update is performed for all branches and the prediction is evaluated against the current measurement to compute a new weight for the branch. Lowprobability branches are then discarded and a measurement update is performed for the remaining branches. The KF can suboptimally be replaced by the EKF or IEKF for the AWGNSSM.

Depending on the application one might also be interested in the mode probability, which can be approximated as

$$
p\left(\delta_{k} \mid \mathcal{Y}_{1: k}\right) \approx \sum_{i \in \mathcal{I}_{k}} w_{k^{\prime}}^{i}
$$

where $\mathcal{I}_{k}=\left\{i \in\{1: M\} \mid \delta_{k}^{i}=\delta_{k}\right\}$. Further, one might be interested in a point estimation of the state, which can be obtained as

$$
\hat{\mathbf{x}}_{k}=\sum_{i=1}^{M} w_{k}^{i} \hat{\mathbf{x}}_{k \mid k}^{i}
$$

\subsubsection{Model Extension}

The modifications required for estimation in the extension of the JMM proposed in Section 3.6.2 is presented in this section. The modifications were first published by the author in [27].

The main difference to the computations in Section 5.4 when adding the additional measurement in (3.50) is that the weight computation becomes

$$
w_{k}^{i} \triangleq p\left(\Delta_{1: k}^{i} \mid \mathcal{Y}_{1: k}, \mathcal{Z}_{1: k}\right)=\frac{p\left(\Delta_{1: k}^{i}\right) p\left(\mathcal{Y}_{1: k}, \mathcal{Z}_{1: k} \mid \Delta_{1: k}^{i}\right)}{p\left(\mathcal{Y}_{1: k}, \mathcal{Z}_{1: k}\right)}
$$




\section{Algorithm 5.7: Filter Bank}

Input: $\overline{\mathbf{x}}_{0}, \mathbf{P}_{0}$ and $\mathbf{y}_{j}$ for $j \in \mathcal{J}$.

Initialization

$$
\Delta_{1: 0}^{i}=\varnothing, \hat{\mathbf{x}}_{0 \mid 0}^{i}=\overline{\mathbf{x}}_{0}, \mathbf{P}_{0 \mid 0}=\mathbf{P}_{0} \text { and } w_{0}^{i}=|\mathcal{S}| / M \text { for } i \in\{1: M /|\mathcal{S}|\} \text {. }
$$

for $k=1, \ldots, K$ do

Sequence Splitting $(i=1, \ldots, M /|\mathcal{S}|)$

for $j=0, \ldots,|\mathcal{S}|-1$ do

$$
\Delta_{1: k}^{i+j \cdot M /|\mathcal{S}|} \leftarrow\left\{\Delta_{1: k-1}^{i}, j\right\}
$$

end for

Time Update $(i=1, \ldots, M)$

Perform time update in Algorithm 5.2 for sequence $\Delta_{1: k}^{i}$ to compute $\hat{\mathbf{x}}_{k \mid k-1}^{i}$ and $\mathbf{P}_{k \mid k-1}^{i}$.

Weight Update $(i=1, \ldots, M)$

$\tilde{w}_{k}^{i} \leftarrow w_{k-1}^{i} \Pi_{k}^{\delta_{k-1}^{i}, \delta_{k-1}^{i}} \mathcal{N}\left(\mathbf{y}_{k} \mid \hat{\mathbf{y}}_{k}^{i}, \mathbf{S}_{k}^{i}\right)$

$w_{k}^{i} \leftarrow \frac{\tilde{w}_{k}^{i}}{\sum_{j=1}^{M} \tilde{w}_{k}^{j}}$

\section{Pruning}

Sort the the weights $w_{k}^{i}$ in descending order, reorder the components accordingly and keep the data for the first $M /|\mathcal{S}|$ sequences.

Measurement Update $(i=1, \ldots, M /|\mathcal{S}|)$

Perform measurement update in Algorithm 5.2 for sequence $\Delta_{1: k}^{i}$ to compute $\hat{\mathbf{x}}_{k \mid k}^{i}$ and $\mathbf{P}_{k \mid k}^{i}$.

end for

Output: $\hat{\mathbf{x}}_{k \mid k^{\prime}}^{i} \mathbf{P}_{k \mid k^{\prime}}^{i} \Delta_{1: k}^{i}$ and $w_{k}^{i}$ for $k \in \mathcal{K}$ and $i \in\{1: M /|\mathcal{S}|\}$. 


$$
\begin{aligned}
& =\frac{p\left(\Delta_{1: k-1}^{i}\right) p\left(\mathcal{Y}_{1: k-1}, \mathcal{Z}_{1: k-1} \mid \Delta_{1: k}^{i}\right)}{p\left(\mathcal{Y}_{1: k-1}, \mathcal{Z}_{1: k-1}\right)} \cdot p\left(\delta_{k}^{i} \mid \delta_{k-1}^{i}\right) . \\
& \frac{p\left(\mathbf{y}_{k}, \mathbf{z}_{k} \mid \mathcal{Y}_{1: k-1}, \mathcal{Z}_{1: k-1}, \Delta_{1: k}^{i}\right)}{p\left(\mathbf{y}_{k}, \mathbf{z}_{k} \mid \mathcal{Y}_{1: k-1}, \mathcal{Z}_{1: k-1}\right)} \\
& =w_{k-1}^{i} \boldsymbol{\Pi}_{k}^{\delta_{k-1}^{i}, \delta_{k-1}^{i}} \frac{p\left(\mathbf{z}_{k} \mid \delta_{k}^{i}\right) \int_{\mathbf{x}_{k}} p\left(\mathbf{y}_{k} \mid \mathbf{x}_{k}, \delta_{k}^{i}\right) p\left(\mathbf{x}_{k} \mid \mathcal{Y}_{1: k-1}, \Delta_{1: k}^{i}\right) d \mathbf{x}_{k}}{p\left(\mathbf{y}_{k}, \mathbf{z}_{k} \mid \mathcal{Y}_{1: k-1}, \mathcal{Z}_{1: k-1}\right)},
\end{aligned}
$$

adding another factor to the weight-update product. As in (5.28) for the general model the computation simplifies for an LGSSM,

$$
w_{k}^{i} \propto w_{k-1}^{i} \boldsymbol{\Pi}_{k}^{\delta_{k}^{i}, \delta_{k-1}^{i}} \mathcal{N}\left(\mathbf{y}_{k} \mid \hat{\mathbf{y}}_{k}^{i}, \mathbf{S}_{k}^{i}\right) p\left(\mathbf{z}_{k} \mid \delta_{k}^{i}\right)
$$

The proposed extension is used for estimating the bird takeoff times as presented in Application 5.1. The results for the application are presented as well, with a comparison to a sequence of manually annotated data.

\section{Application 5.1: Bird Mode Estimation}

The primary goal is to determine time and direction of takeoffs, and the secondary goal is to measure the activity of the bird. The time of a takeoff is estimated as a transition from stationary to flight in the JMM in Application 3.4 and the direction is estimated from the states in the JMM.

Together with the models described in Applications 3.4, 3.5 and 4.1 the methods described in this chapter are used to estimate both the current mode of the bird and its position within the funnel, providing sufficient information to extract time and direction of the takeoffs.

An assay was prepared filming four European robins over approximately an hour at six frames per second. Using the image processing methods described in Application 4.5, the position, orientation and blurriness of the birds are extracted. The camera model is estimated for each funnel according to the method described in Application 4.1. Two models are set up as in Applications 3.4 and 3.5, one for the stationary mode with low process noise covariance, low blur level and high probability of being close to the centre of the funnel, and another for the flight mode with opposite characteristics.

The method described in this section together with an IEKF is used to estimate the states and modes for the birds. The advantage of using the IEKF is mainly that the position of the bird in world coordinates is more accurate compared to an EKF. The estimated mode over time for each bird is shown in Figure 5.2. The estimation is at times noisy, so heuristics are applied to filter out short mode intervals and movements that are too small to be takeoffs. Each transition from stationary to flight mode is then extracted as a takeoff, shown in Figure 5.3.

The direction is extracted by a basic method, computed as the angle between the position before and after a transition. Due to the low frame rate, however, the particular frames might not be representative of the takeoff. An improved method therefore considers the position of the bird in a number of frames around the transition where the angle of the trajectory only changes by 

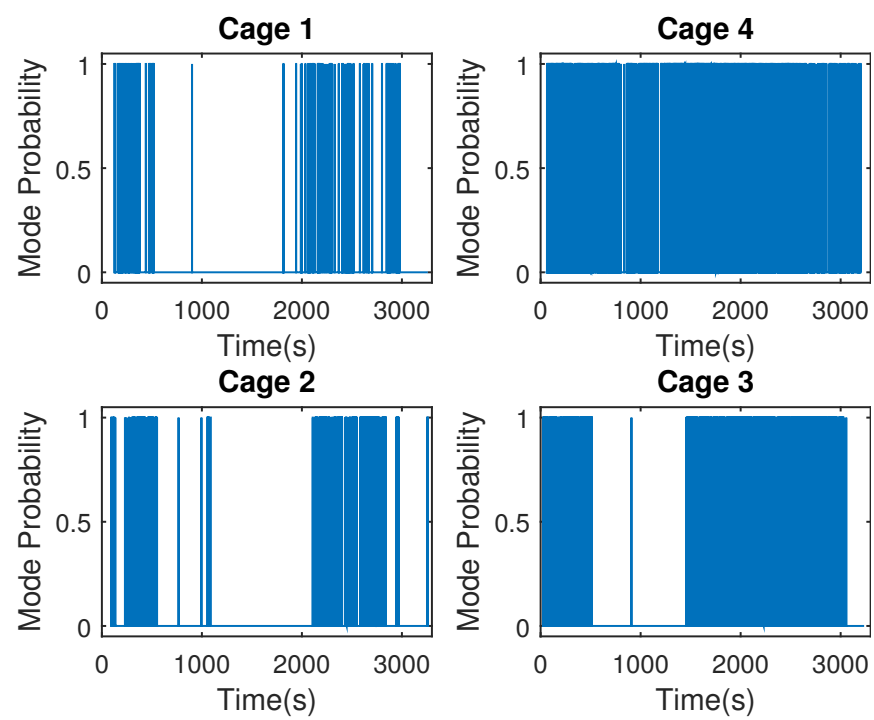

Figure 5.2: Mode probability for flight mode over time for all four birds.
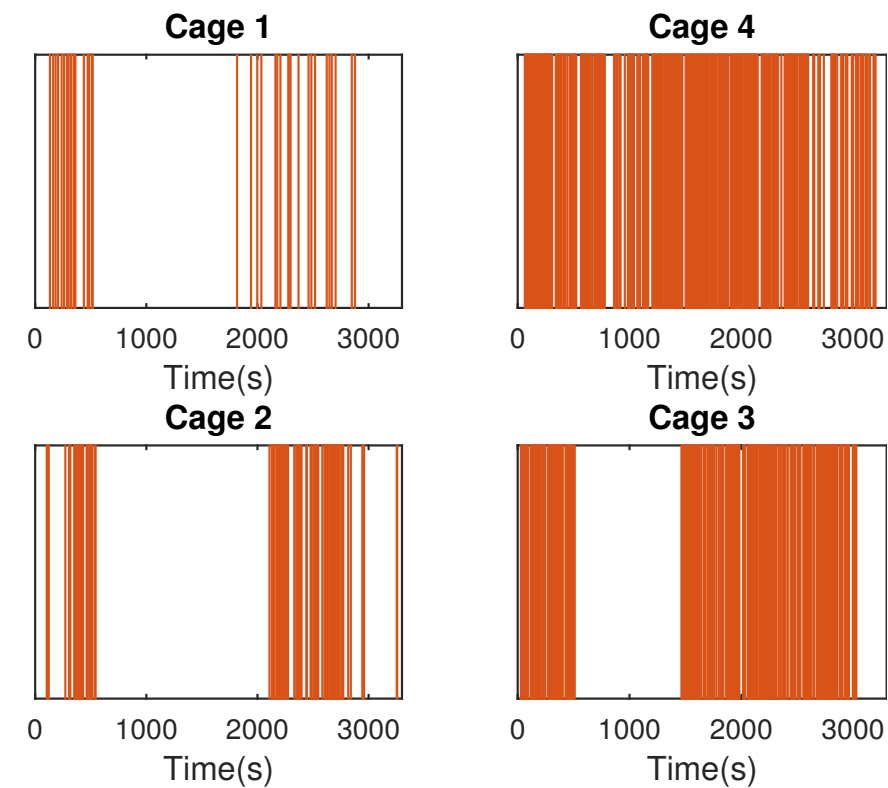

Figure 5.3: Estimated times of takeoffs for all four birds after heuristic filtering. 

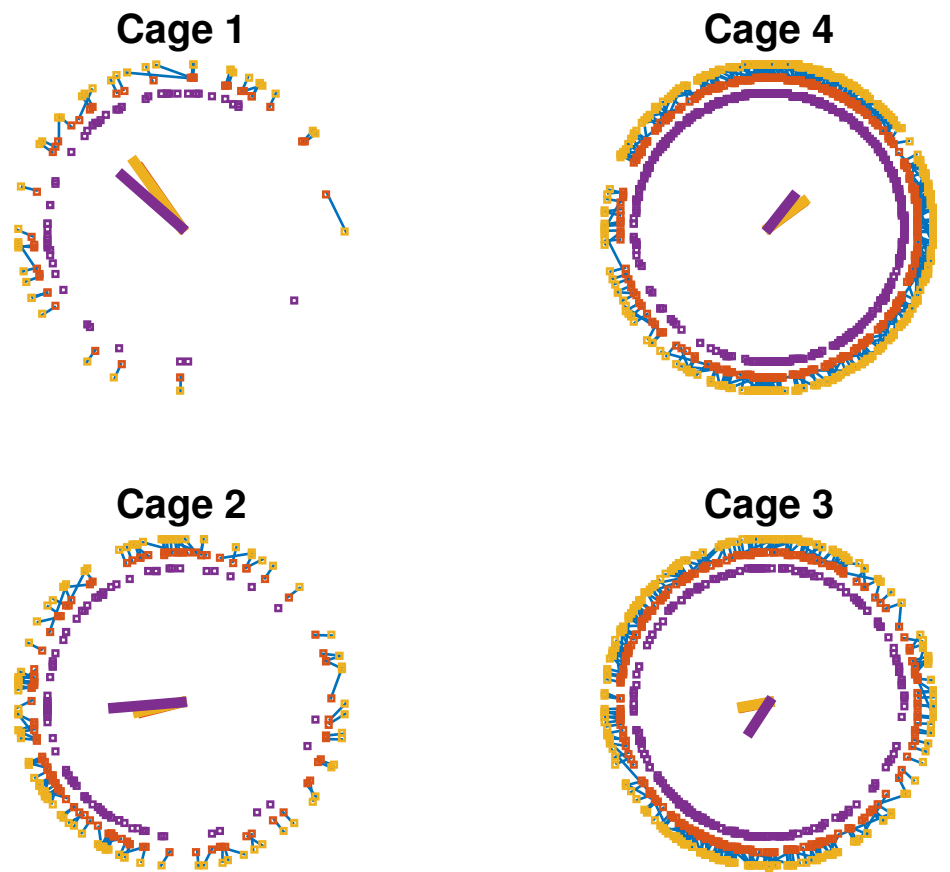

Figure 5.4: The estimates for the basic and improved methods for extracting takeoff directions are shown as the outer and the middle dots, respectively, with a line connecting dots extracted from the same takeoff. The inner circle shows the direction from the centre in which the bird left the inner circle, completely disregarding the mode estimates. The lines in the centre indicate average directions of takeoff for the methods, where a long line is more significant than a short line.

a reasonable amount. A third, simpler, method that does not require a filter is also considered for comparison. In this method a takeoff is estimated every time the bird leaves the inner circle. The direction is extracted as the angle between the estimated position outside the inner circle and the centre of the funnel.

Figure 5.4 shows the result of the considered methods, as well as an average takeoff direction, where all methods result in similar averages. Figure 5.5 shows an example of a takeoff drawn into the original video using the improved method.

The proposed method is compared to a traditional method and a number of modern methods in [27], and is deemed to be a viable solution to automate the biological experiment. In addition to providing similar data as traditional methods, the proposed method also provides a temporal dimension and experiments can be conducted for a longer time without disturbing the birds. An additional advantage is that the birds can potentially be held in larger cages with food and water, allowing, from an ethical point of view, for even longer experiments. The biologists are also interested in the activity of the birds, which can, e.g., be ex- 

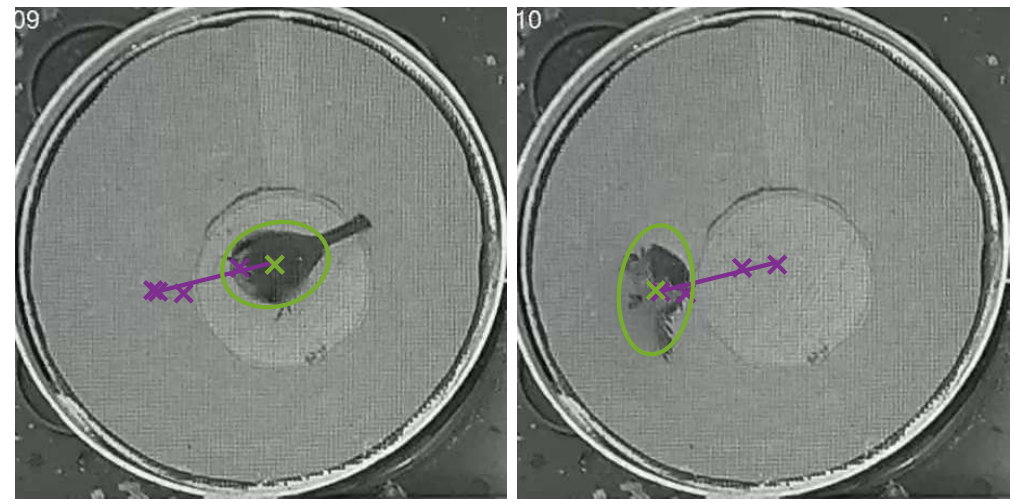

Figure 5.5: A takeoff estimated over several frames using the improved method. Bird position and extent is in green, positions included in the takeoff are magenta and the line is the estimated takeoff direction.

tracted from the estimated modes or from the estimated states by computing the distance travelled by the bird.

The first quarter of the experiment has been manually annotated, so the time and direction of all takeoffs during this period are available. The takeoffs are in this case defined slightly differently as the maximum distance of the beak from the funnel centre in image coordinates, making a comparison of the orientations for individual takeoffs less informative. A disadvantage of the manual method is that when a bird pursues a takeoff for a long time, bouncing against the net, it is likely to result in an estimated takeoff direction that was initially not intended by the bird. However, takeoff times should not differ too much for individual takeoffs and the average takeoff directions should be comparable between the methods.

A takeoff time estimated using the proposed method is matched to a takeoff in the manually annotated data if the time difference is low. The results for matching takeoff times between the methods are shown in Table 5.1. A large percentage of all takeoffs detected by the proposed method are also annotated manually, but less than half of the manually annotated takeoffs are detected by the proposed method. The results are better, however, if the fourth cage is disregarded. The fourth cage has particularly many takeoffs due to an active bird, corroborated by Figures 5.2 and 5.3. An active bird spends less time scanning in the centre of the funnel, making it more difficult to separate individual takeoffs. The policy used in the manual method for estimating takeoffs also includes bounces back through the inner circle, which are more likely to occur for an active bird and are of less interest considering the bird did not intentionally choose the direction. Such takeoffs are however discarded by the proposed method, which could be an explanation for the large discrepancy for the fourth cage.

Individual takeoffs that are matched between the methods are shown in Figure 5.6. Since takeoff directions are defined differently, these methods show 
Table 5.1: In the two right columns are the number of estimated takeoffs for the manual method and the proposed method respectively. In the second column are the number of matches between them. The percentage indicates the proportion matched.

\begin{tabular}{cccccc}
\hline Cage & Matches & \multicolumn{2}{c}{ Manual Method } & \multicolumn{2}{c}{ Proposed Method } \\
\hline 1 & 14 & 21 & $67 \%$ & 18 & $78 \%$ \\
2 & 22 & 35 & $63 \%$ & 31 & $71 \%$ \\
3 & 60 & 87 & $69 \%$ & 66 & $91 \%$ \\
4 & 42 & 190 & $22 \%$ & 51 & $82 \%$ \\
\hline All & 138 & 333 & $41 \%$ & 166 & $83 \%$ \\
\hline
\end{tabular}

many severe mismatches in direction, but as can be seen, the average directions, shown as lines in the centre of the circle, are similar between the methods. The practical use of the extension to the JMM on real data has been demonstrated. This concludes the bird application.

\subsection{Extended Target Model Filter}

As presented in [107], the approach for estimation in the extended target model in Section 3.7 is similar to estimation for the ordinary SSM presented in (5.1) and (5.2). The difference is in an additional consideration for an unknown covariance matrix. Given measurements $\mathcal{Y}_{1: k}=\left\{\mathcal{Y}_{j}\right\}_{j \in\{1: k\} \cap \mathcal{J}}$ and the model in Section 3.7, the posterior $p\left(\mathbf{x}_{k}, \mathbf{X}_{k} \mid \mathcal{Y}_{1: k}\right)$ of the kinematic state $\mathbf{x}_{k}$ and extent $\mathbf{X}_{k}$ are sought.

Using Bayes' theorem and the Markov property, the time update is given by

$$
\begin{aligned}
& p\left(\mathbf{x}_{k}, \mathbf{x}_{k} \mid \mathcal{Y}_{1: k-1}\right)= \int p\left(\mathbf{x}_{k}, \mathbf{X}_{k} \mid \mathbf{x}_{k-1}, \mathbf{X}_{k-1}, \mathcal{Y}_{k-1}\right) . \\
& p\left(\mathbf{x}_{k-1}, \mathbf{X}_{k-1} \mid \mathcal{Y}_{k-1}\right) \mathrm{d} \mathbf{x}_{k-1} \mathrm{~d} \mathbf{X}_{k-1} \\
&=\int p\left(\mathbf{x}_{k} \mid \mathbf{x}_{k-1}, \mathbf{X}_{k}\right) \cdot p\left(\mathbf{X}_{k} \mid \mathbf{X}_{k-1}\right) \cdot p\left(\mathbf{x}_{k-1} \mid \mathbf{X}_{k-1}, \mathcal{Y}_{k-1}\right) . \\
& p\left(\mathbf{X}_{k-1} \mid \mathcal{Y}_{k-1}\right) \mathrm{d} \mathbf{x}_{k-1} \mathrm{~d} \mathbf{X}_{k-1} .
\end{aligned}
$$

and the measurement update by

$$
\begin{aligned}
p\left(\mathbf{x}_{k}, \mathbf{x}_{k} \mid \mathcal{Y}_{1: k}\right) & \propto p\left(\mathcal{Y}_{k}, N_{j} \mid \mathbf{x}_{k}, \mathbf{x}_{k}, \mathcal{Y}_{k-1}\right) \cdot p\left(\mathbf{x}_{k}, \mathbf{x}_{k} \mid \mathcal{Y}_{k-1}\right) \\
& =p\left(\mathcal{Y}_{k} \mid N_{j}, \mathbf{x}_{k}, \mathbf{x}_{k}\right) \cdot p\left(N_{k} \mid \mathbf{x}_{k}, \mathbf{x}_{k}\right) \cdot p\left(\mathbf{x}_{k}, \mathbf{x}_{k} \mid \mathcal{Y}_{k-1}\right) \\
& \propto \prod_{i=1}^{N_{k}} p\left(\mathbf{y}_{k}^{i} \mid \mathbf{x}_{k}, \mathbf{x}_{k}\right) \cdot p\left(\mathbf{x}_{k} \mid \mathbf{x}_{k}, \mathcal{Y}_{k-1}\right) p\left(\mathbf{x}_{k} \mid \mathcal{Y}_{k-1}\right)
\end{aligned}
$$

All distributions required for (5.33) and (5.34) are given in Section 3.7. Since conjugate prior distributions [70] are chosen for the kinematic state and the extent the posterior distributions can be computed in closed form [107]. 
Cage 1

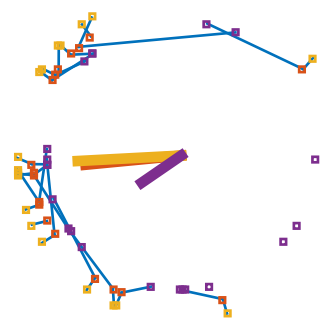

Cage 2

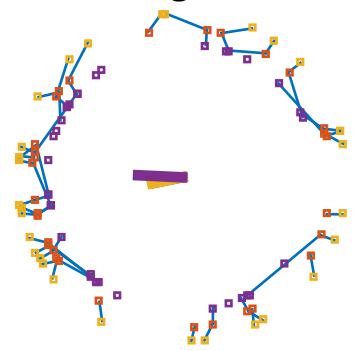

Cage 4

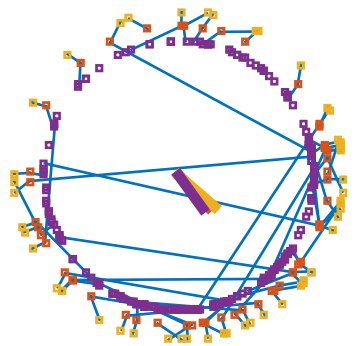

Cage 3

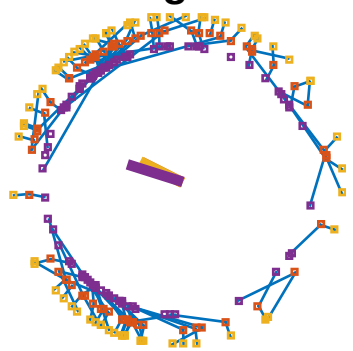

Figure 5.6: Going inwards, the estimated takeoff directions using the basic, improved and manual methods are shown as dots. The connection between the dots indicate that the time of the takeoffs are similar and therefore represent the same takeoff.

The prediction and filtering distributions for the model in (3.55)-(3.59) are computed, using derivations in [107], as

$$
\begin{aligned}
p\left(\mathbf{x}_{k}, \mathbf{x}_{k} \mid \mathcal{Y}_{1: k-1}\right) & =\mathcal{N}\left(\mathbf{x}_{k} \mid \hat{\mathbf{x}}_{k \mid k-1}, \mathbf{P}_{k \mid k-1} \otimes \mathbf{X}_{k}\right) \cdot \mathcal{I} \mathcal{W}\left(\mathbf{X}_{k} \mid v_{k \mid k-1}, \mathbf{V}_{k \mid k-1}\right) \\
p\left(\mathbf{x}_{k}, \mathbf{x}_{k} \mid \mathcal{Y}_{1: k}\right) & =\mathcal{N}\left(\mathbf{x}_{k} \mid \hat{\mathbf{x}}_{k \mid k}, \mathbf{P}_{k \mid k} \otimes \mathbf{X}_{k}\right) \cdot \mathcal{I} \mathcal{W}\left(\mathbf{X}_{k} \mid v_{k \mid k}, \mathbf{V}_{k \mid k}\right)
\end{aligned}
$$

where $\mathbf{P}_{k \mid k-1} \in S_{++}^{s}$ and $\mathbf{P}_{k \mid k} \in S_{++}^{s}$. The time update is performed as

$$
\begin{aligned}
\hat{\mathbf{x}}_{k \mid k-1} & =\left(\tilde{\mathbf{F}}_{k} \otimes \mathbf{I}_{p}\right) \hat{\mathbf{x}}_{k-1 \mid k-1}, \\
\mathbf{P}_{k \mid k-1} & =\tilde{\mathbf{F}}_{k} \mathbf{P}_{k-1 \mid k-1} \tilde{\mathbf{F}}_{k}^{\top}+\tilde{\mathbf{Q}}_{k}, \\
v_{k \mid k-1} & =e^{-T_{k} / \tau} v_{k-1}, \\
\mathbf{V}_{k \mid k-1} & =\frac{e^{-T_{k} / \tau} v_{k-1 \mid k-1}-2 p-2}{v_{k-1 \mid k-1}-2 p-2} \mathbf{V}_{k-1 \mid k-1},
\end{aligned}
$$

and the measurement update as

$$
\begin{aligned}
\hat{\mathbf{x}}_{k \mid k} & =\hat{\mathbf{x}}_{k \mid k-1}+\left(\tilde{\mathbf{K}}_{k} \otimes \mathbf{I}_{p}\right)\left(\mathbf{y}_{k}-\left(\tilde{\mathbf{H}}_{k} \otimes \mathbf{I}_{p}\right) \hat{\mathbf{x}}_{k \mid k-1}\right), \\
\mathbf{P}_{k \mid k} & =\mathbf{P}_{k \mid k-1}-\tilde{\mathbf{K}}_{k} S_{k} \tilde{\mathbf{K}}_{k}^{\top},
\end{aligned}
$$




$$
\begin{aligned}
v_{k \mid k} & =v_{k \mid k-1}+N_{k} \\
\mathbf{V}_{k \mid k} & =\mathbf{V}_{k \mid k-1}+\mathbf{N}_{k}+\mathbf{Y}_{k},
\end{aligned}
$$

where

$$
\begin{aligned}
\tilde{\mathbf{K}}_{k} & =\mathbf{P}_{k \mid k-1} \tilde{\mathbf{H}}_{k}^{\top} S_{k}^{-1}, \\
S_{k} & =\tilde{\mathbf{H}}_{k} \mathbf{P}_{k \mid k-1} \tilde{\mathbf{H}}_{k}^{\top}+\frac{1}{N_{k}}, \\
\mathbf{y}_{k} & =\frac{1}{N_{k}} \sum_{i=1}^{N_{k}} \mathbf{y}_{k^{\prime}}^{i} \\
\mathbf{Y}_{k} & =\frac{1}{N_{k}} \sum_{i=1}^{N_{k}}\left(\mathbf{y}_{k}^{i}-\mathbf{y}_{k}\right)\left(\mathbf{y}_{k}^{i}-\mathbf{y}_{k}\right)^{\top}, \\
\mathbf{N}_{k} & =S_{k}^{-1}\left(\mathbf{y}_{k}-\left(\tilde{\mathbf{H}}_{k} \otimes \mathbf{I}_{p}\right) \hat{\mathbf{x}}_{k \mid k-1}\right)\left(\mathbf{y}_{k}-\left(\tilde{\mathbf{H}}_{k} \otimes \mathbf{I}_{p}\right) \hat{\mathbf{x}}_{k \mid k-1}\right)^{\top} .
\end{aligned}
$$

The time and measurement updates for the kinematic state vector are similar to those in the KF. Estimates of the kinematic state covariance and the covariance matrix representing the extent are computed as [77, 107]

$$
\begin{aligned}
\hat{\mathbf{P}}_{k} & =\frac{\mathbf{P}_{k \mid k} \otimes \mathbf{V}_{k \mid k}}{v_{k \mid k}+s-s p-2^{\prime}}, \\
\hat{\mathbf{X}}_{k} & =\frac{\mathbf{V}_{k \mid k}}{v_{k \mid k}-2 p-2^{\prime}}
\end{aligned}
$$

for $v_{k \mid k}$ such that the denominators are positive.

The covariance matrix representing the extent is estimated with the assumption that the measurements are Gaussian distributed over the target. However, a uniform distribution is a more reasonable assumption for measurements from a camera sensor due to the uniform sampling grid of the pixels. Assuming that the targets are elliptical and that the measurements are uniformly distributed, the elliptical extent is computed as the solutions of $\mathbf{z}$ in the inequality

$$
\left(\mathbf{z}-\left(\mathbf{H}_{k} \otimes \mathbf{I}_{p}\right) \hat{\mathbf{x}}_{k \mid k}\right)^{\top} \hat{\mathbf{X}}_{k \mid k}^{-1}\left(\mathbf{z}-\left(\mathbf{H}_{k} \otimes \mathbf{I}_{p}\right) \hat{\mathbf{x}}_{k \mid k}\right) \leq 4
$$

using Proposition 5.3. A simple method to generate a path along the edge is to Cholesky factorize the covariance matrix and perform an affine transformation on a path generated along the unit circle.

Proposition 5.3: Given is a stochastic variable with a uniform distribution

$$
\mathbf{x} \sim p(\mathbf{x})= \begin{cases}A_{\mathcal{C}}^{-1}, & \text { if } \mathbf{x} \in \mathcal{C}, \\ 0, & \text { otherwise }\end{cases}
$$

over an ellipse defined by

$$
\mathcal{C}=\left\{\mathbf{x} \in \mathbb{R}^{2} \mid(\mathbf{x}-\mathbf{b})^{\top} \mathbf{C}^{-1}(\mathbf{x}-\mathbf{b}) \leq 1\right\},
$$


where $\mathbf{C}=\mathbf{A A}^{\top}$ is a symmetric and positive definite matrix, $\mathbf{b}$ is the centroid of the ellipse and $A_{\mathcal{C}}$ is the area of the ellipse.

The mean and covariance of the stochastic variable are then given by

$$
\mathbf{E}(\mathbf{x})=\mathbf{b} \quad \text { and } \quad \operatorname{Cov}(\mathbf{x})=\frac{\mathbf{C}}{4} .
$$

Proof: The proof is presented in Appendix A.4.

The method described in this section is applied in the savannah application. However, considering the association uncertainty in the application the presentation is deferred to Application 6.1 in Chapter 6.

\subsection{Uncertain Timestamp Model Smoother}

Conditional on the uncertain timestamp, the UTM defined in Section 3.8 reduces to an LGSSM, for which the states can be estimated using the RTSS [152]. This is used to handle the uncertainty of the timestamp and relevant posterior distributions and estimators are derived in Sections 5.6.1 and 5.6.2, respectively. The results for the simple uncertain time scenario and the analysis in Application 5.7 were first published by the author in [182]. The derivations for the posterior distributions, the point estimators and the simulated scenario have been accepted for publication in [183].

\subsubsection{Posterior Distributions}

The posterior distribution of the state in the UTM is computed by marginalization of the joint posterior distribution of the states and timestamps. It is given by the compound distribution

$$
p(\mathbf{x}(\cdot) \mid \mathcal{Y}, \mathcal{Z})=\int_{\mathcal{T}} p(\mathbf{x}(\cdot) \mid \mathcal{T}, \mathcal{Y}, \mathcal{Z}) \cdot p(\mathcal{T} \mid \mathcal{Y}, \mathcal{Z}) d \mathcal{T},
$$

where the integration is over all possible timestamps $\mathcal{T}$. The first factor in the integrand is recognized as the posterior distribution of the state, conditioned on $\mathcal{T}$. This distribution can be computed using the various methods discussed in Section 5.3, resulting in

$$
p(\mathbf{x}(\cdot) \mid \mathcal{T}, \mathcal{Y}, \mathcal{Z})=\mathcal{N}\left(\mathbf{x}(\cdot) \mid \hat{\mathbf{x}}^{\mathcal{T}}(\cdot), \mathbf{P}^{\mathcal{T}}(\cdot)\right),
$$

where the superscript indicates which timestamps are used. The second factor in the integrand in (5.44) is the posterior distribution of the uncertain timestamps $\mathcal{T}$, which can be computed up to a proportionality constant using Bayes' theorem and the independence assumption [159] as

$$
w_{\mathcal{T}} \triangleq p(\mathcal{T} \mid \mathcal{Y}, \mathcal{Z}) \propto p(\mathcal{Z} \mid \mathcal{T}, \mathcal{Y}) p(\mathcal{T})
$$




$$
=\prod_{i \in \mathcal{I}}\left[p\left(\mathbf{z}_{i} \mid \mathcal{T}_{1: i}, \mathcal{Y}, \mathcal{Z}_{1: i-1}\right) p\left(\tau_{i} \mid \mathcal{T}_{1: i-1}\right)\right]
$$

where the subscript notation $a: b=\{i\}_{i=a}^{b}$ is used to index a subset of the elements. The likelihood of observation $\mathbf{z}_{i}$ given a subset of the other observations and their timestamps is computed by marginalizing the joint posterior distribution of the observation $\mathbf{z}_{i}$ and the state $\mathbf{x}\left(\tau_{i}\right)$ at time $\tau_{i}$ as

$$
\begin{aligned}
p\left(\mathbf{z}_{i} \mid \mathcal{T}_{1: i}, \mathcal{Y}, \mathcal{Z}_{1: i-1}\right)=\int p\left(\mathbf{z}_{i}, \mathbf{x}\left(\tau_{i}\right) \mid \mathcal{T}_{1: i}, \mathcal{Y}, \mathcal{Z}_{1: i-1}\right) d \mathbf{x}\left(\tau_{i}\right) \\
\quad=\int p\left(\mathbf{z}_{i} \mid \mathbf{x}\left(\tau_{i}\right), \tau_{i}\right) \cdot p\left(\mathbf{x}\left(\tau_{i}\right) \mid \mathcal{Y}, \mathcal{Z}_{1: i-1}, \mathcal{T}_{1: i-1}\right) d \mathbf{x}\left(\tau_{i}\right) \\
\quad=\mathcal{N}\left(\mathbf{z}_{i} \mid \mathbf{H}_{i}^{z} \hat{\mathbf{x}}^{\mathcal{T}_{1: i-1}}\left(\tau_{i}\right), \mathbf{H}_{i}^{z} \mathbf{P}^{\mathcal{T}_{1: i-1}}\left(\tau_{i}\right)\left(\mathbf{H}_{i}^{z}\right)^{\top}+\mathbf{R}_{i}^{z}\right) \\
=\mathcal{N}\left(\mathbf{z}_{i} \mid \hat{\mathbf{z}}_{i}^{\mathcal{T}_{1: i-1}}\left(\tau_{i}\right), \mathbf{S}_{i}^{\mathcal{T}_{1: i-1}}\left(\tau_{i}\right)\right),
\end{aligned}
$$

where $\int \mathcal{N}(\mathbf{a} \mid \mathbf{A} \mathbf{b}, \mathbf{B}) \mathcal{N}(\mathbf{b} \mid \mathbf{c}, \mathbf{C}) d \mathbf{b}=\mathcal{N}\left(\mathbf{a} \mid \mathbf{A c}, \mathbf{A C A}^{\top}+\mathbf{B}\right)$ is used in the third equality. The last equality defines a convenient notation for the mean and covariance of the prediction of $\mathbf{z}_{i}$ at time $\tau_{i}$ given the observations $\mathcal{Z}_{1: i-1}$, timestamps $\mathcal{T}_{1: i-1}$ and all the measurements $\mathcal{Y}$.

Computation of (5.46) is performed stepwise. First, the smoothed posterior $p(\mathbf{x}(\cdot) \mid \mathcal{Y})$ is computed for the measurements $\mathcal{Y}$. The likelihood of each observation is computed in turn at the given timestamp and the observation and timestamp are sequentially added as conditional variables to the posterior distribution. The order in which the observations are added is unimportant. The result is the product of all the computed likelihoods and the joint prior of the timestamps. The joint prior $p(\mathcal{T})$ can also be computed sequentially using the chain rule. Inserting (5.45) and (5.46) into (5.44) gives the compound distribution

$$
p(\mathbf{x}(\cdot) \mid \mathcal{Y}, \mathcal{Z})=\int_{\mathcal{T}} w_{\mathcal{T}} \mathcal{N}\left(\mathbf{x}(\cdot) \mid \hat{\mathbf{x}}^{\mathcal{T}}(\cdot), \mathbf{P}^{\mathcal{T}}(\cdot)\right) d \mathcal{T}
$$

where $w_{\mathcal{T}}$ can be seen as the weight of the hypothesis of timestamps $\mathcal{T}$.

This class of distributions occurs in many fields of applications [103, 155], which has resulted in a number of established methods for computation. For a discrete timestamp prior, the posterior reduces to a Gaussian mixture distribution since $w_{\mathcal{T}}$ cannot have more support than $p(\mathcal{T})$. However, the posterior is difficult to compute analytically for more general priors. In such cases the posterior distribution needs to be approximated or computed numerically. The main challenges lie in obtaining a representative approximation of the timestamp posterior distribution in (5.46) and obtaining a useful and informative presentation of the continuous-time state posterior distribution in (5.44).

The derivations in this section are used to compute the posterior distributions for the simple uncertain time scenario in Application 5.2. 


\section{Application 5.2: Simple Uncertain Time Posterior Distributions}

The posterior distributions derived in this section for a UTM are computed for the two cases of the simple scenario defined in Application 3.7.

The marginal posterior distribution $p\left(x_{k} \mid y_{1}, z_{1}\right)$ is computed using (5.48) and is shown in Figure 5.7 for the two cases. The distribution $p\left(\tau_{1} \mid y_{1}, z_{1}\right)$ is computed using (5.46) and is shown, together with the prior distribution $p\left(\tau_{1}\right)$, in Figure 5.8 for the two cases. In addition, $\max _{\mathcal{X}} p\left(\mathcal{X}, \tau_{1} \mid y_{1}, z_{1}\right)$ is also computed and displayed for comparison.

Assuming the underlying system to be continuous and the measurements and the observation to be noise free, the intermediate value theorem [1] for the first model already gives the information that $x\left(\tau_{1}\right)=z_{1}$ for a continuous $\tau_{1}$, so at a first glance the observation does not seem to add much new information. However, since $\tau_{1}$ is random, the state is more likely to be in the vicinity of the observation, explaining the shape of the posterior distribution in Figure 5.7a. For the second model in Figure $5.7 \mathrm{~b}$ the prior distribution is more informative and the observation is also not one guaranteed by the intermediate value theorem, thus it provides more information than in the first case.

In Figure 5.8b the distributions do not differ much, but are slightly shifted to account for the observation being closer to the last measurement than the first measurement. Figure 5.8a is less intuitive. The unusual shape of $p\left(\tau_{1} \mid y_{1}, z_{1}\right)$ is further analysed in Application 5.7, where the distribution is compared to $\max _{\mathcal{X}} p\left(\mathcal{X}, \tau_{1} \mid y_{1}, z_{1}\right)$. The MMSE estimator is derived in Application 5.3.

\subsubsection{Point Estimators}

Point estimators [103] are used to provide useful and informative presentations of the posterior distributions in Section 5.6.1. The minimum mean squared error (MMSE) estimator and the maximum a posteriori (MAP) estimator [103] are derived for the states as well as the MAP estimator for the timestamps and the joint maximum a posteriori (JMAP) estimator for the timestamps and states.

\section{Minimum Mean Squared Error Estimator}

The MMSE estimator, derived in [103], for the state posterior distribution in (5.48) is the posterior mean given by

$$
\begin{aligned}
\hat{\mathbf{x}}^{\mathrm{MMSE}}(\cdot) & =E_{\mathbf{x}(\cdot) \mid \mathcal{Y}, \mathcal{Z}}[\mathbf{x}(\cdot)]=E_{\mathcal{T} \mid \mathcal{Y}, \mathcal{Z}}\left[E_{\mathbf{x}(\cdot) \mid \mathcal{T}, \mathcal{Y}, \mathcal{Z}}[\mathbf{x}(\cdot)]\right] \\
& =\int_{\mathcal{T}} w_{\mathcal{T}} E_{\mathbf{x}(\cdot) \mid \mathcal{T}, \mathcal{Y}, \mathcal{Z}}[\mathbf{x}(\cdot)] d \mathcal{T}=\int_{\mathcal{T}} w_{\mathcal{T}} \hat{\mathbf{x}}^{\mathcal{T}}(\cdot) d \mathcal{T}
\end{aligned}
$$

For a continuous timestamp prior, the estimator generally needs to be approximated. The uncertainty of the estimator is approximated by the posterior co- 


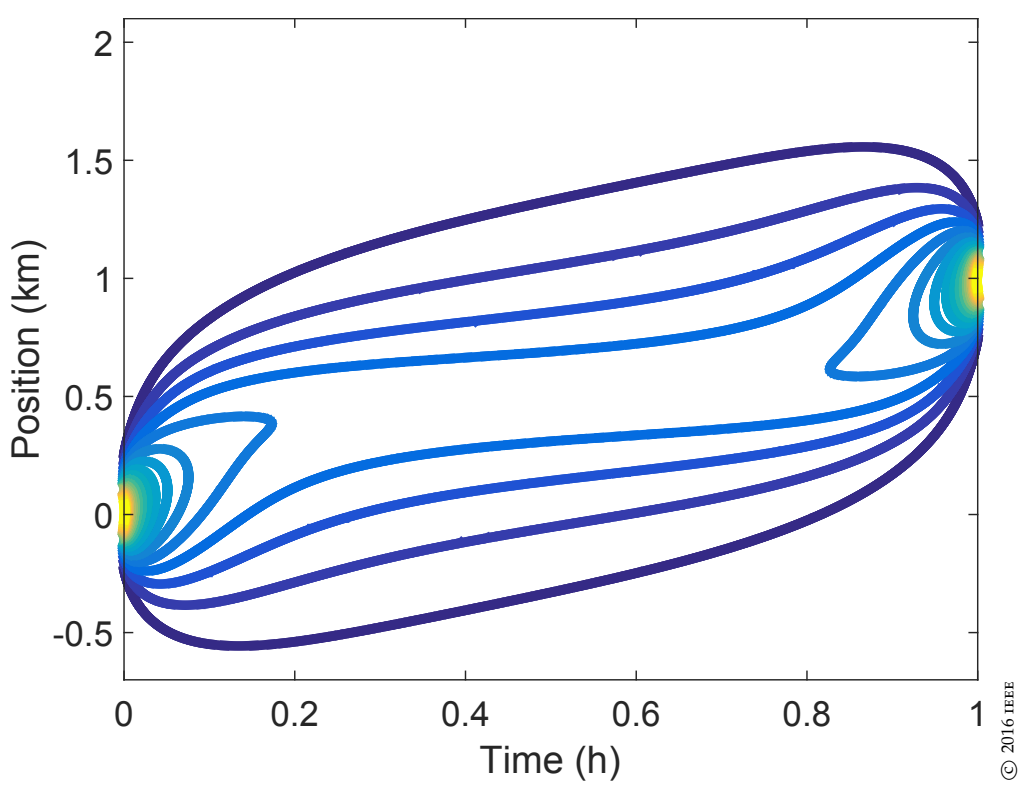

(a) First case.

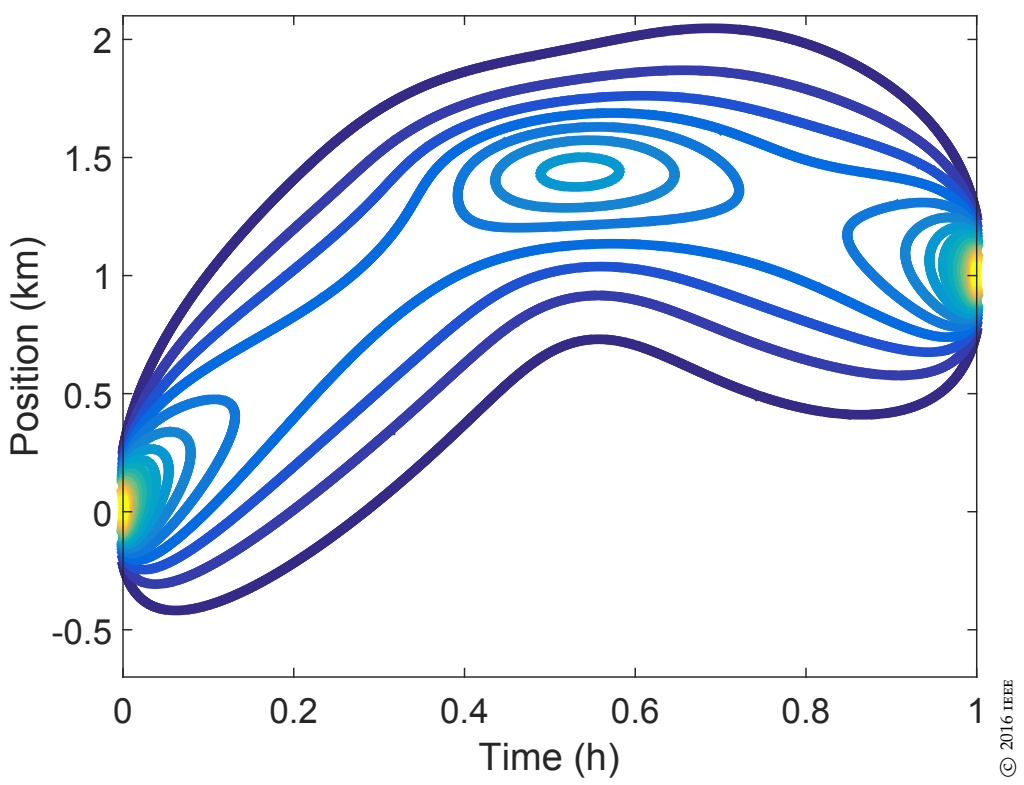

(b) Second case.

Figure 5.7: Contour plots of the posterior distributions for the simple uncertain time scenario. Published in [182]. 


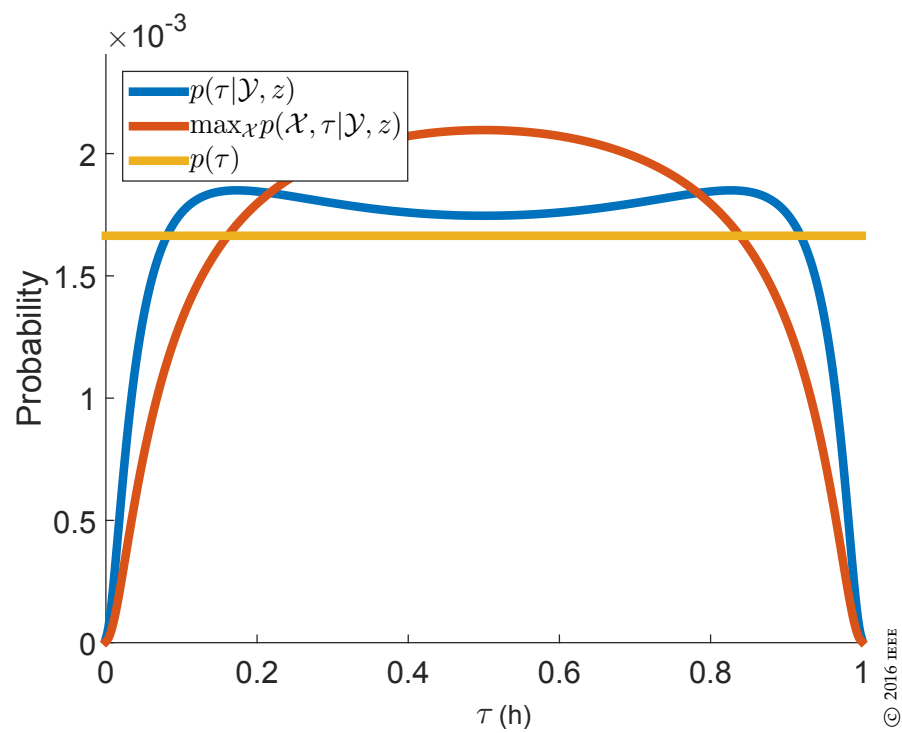

(a) First case.

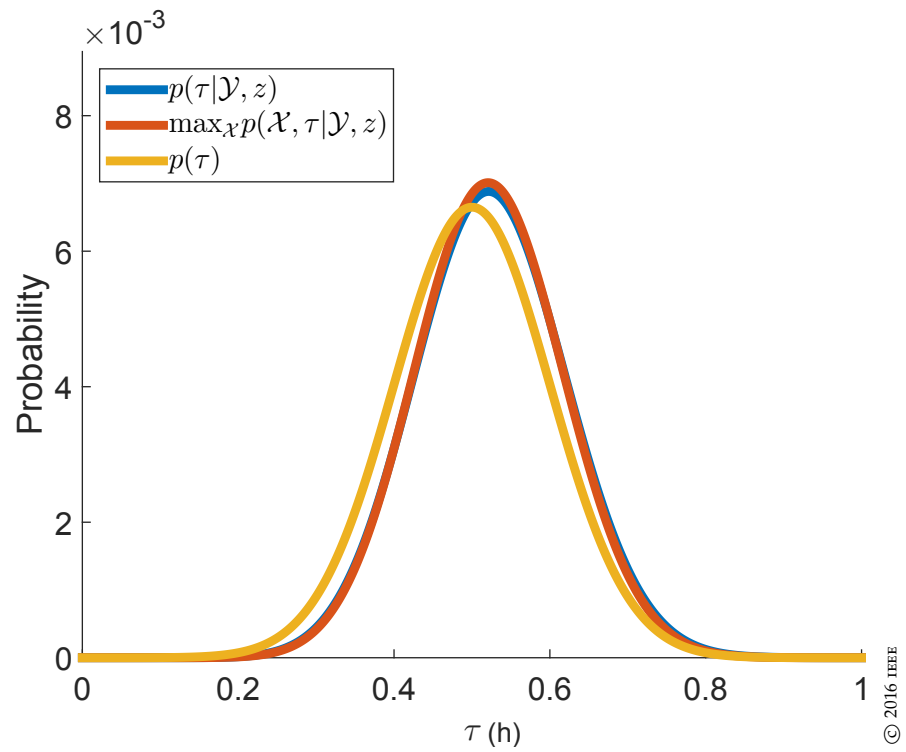

(b) Second case.

Figure 5.8: The distributions $p\left(\tau \mid y_{1}, y_{N}, z\right), \max _{\mathcal{X}} p\left(\mathcal{X}, \tau \mid y_{1}, y_{N}, z\right)$ and $p(\tau)$ for the simple uncertain time scenario. Published in [182]. 
variance as

$$
\mathbf{P}^{\mathrm{MMSE}}(\cdot) \approx \int_{\mathcal{T}} w_{\mathcal{T}}\left(\mathbf{P}^{\mathcal{T}}(\cdot)+\tilde{\mathbf{x}}^{\mathcal{T}}(\cdot)\left(\tilde{\mathbf{x}}^{\mathcal{T}}(\cdot)\right)^{\top}\right) d \mathcal{T},
$$

where $\tilde{\mathbf{x}}^{\mathcal{T}}(\cdot)=\hat{\mathbf{x}}^{\mathcal{T}}(\cdot)-\hat{\mathbf{x}}^{\mathrm{MMSE}}(\cdot)$. The MMSE estimator for the states in the simple uncertain time scenario is presented in Application 5.3.

\section{Application 5.3: Simple Uncertain Time MMSE Estimator}

The MMSE estimate is shown in Figure 5.9 for the two cases together with the uncertainty and the estimate using only measurements with precise timestamps. Further, the MAP estimator is also presented in Application 5.4.

\section{Maximum A Posteriori State Estimator}

The MAP estimator, derived in [103], for the state posterior distribution in (5.48) is given by

$$
\begin{aligned}
\hat{\mathbf{x}}^{\mathrm{MAP}}(\cdot) & =\underset{\mathbf{x}(\cdot)}{\arg \max } p(\mathbf{x}(\cdot) \mid \mathcal{Y}, \mathcal{Z}) \\
& =\underset{\mathbf{x}(\cdot)}{\arg \max } \int_{\mathcal{T}} w_{\mathcal{T}} \mathcal{N}\left(\mathbf{x}(\cdot) \mid \hat{\mathbf{x}}^{\mathcal{T}}(\cdot), \mathbf{P}^{\mathcal{T}}(\cdot)\right) d \mathcal{T} .
\end{aligned}
$$

Unlike the MMSE estimate, the MAP estimate for one instant of time also depends on the other time instants included in the maximization [103]. The MAP estimate at a particular instant of time will therefore differ depending on whether it is computed only for the state $\mathbf{x}(t)$ at that instant, the states $\mathcal{X}$ including other instants or the continuous-time trajectory $\mathbf{x}(\cdot)$. The estimator will in general, even in the case of a discrete timestamp prior, be impossible to compute analytically, so approximate or numerical methods are required.

The MAP estimator for the simple uncertain time scenario is presented in Application 5.4.

\section{Application 5.4: Simple Uncertain Time MAP Estimator}

The MAP estimates of (5.48) for the simple uncertain time scenario, both jointly for the states as $p\left(\mathcal{X} \mid y_{1}, z_{1}\right)$ and independently for each state as $p\left(x_{k} \mid y_{1}, z_{1}\right)$, are presented in Figure 5.10 together with the credible interval for latter. The estimates are computed with the quasi-Newton numerical optimization method [51], without a guarantee of convergence to the global maximum. The MMSE estimator using only measurements with precise timestamps, which is equal to the MAP estimator for this case, is also shown for comparison. A third point estimator is considered in Application 5.6. 


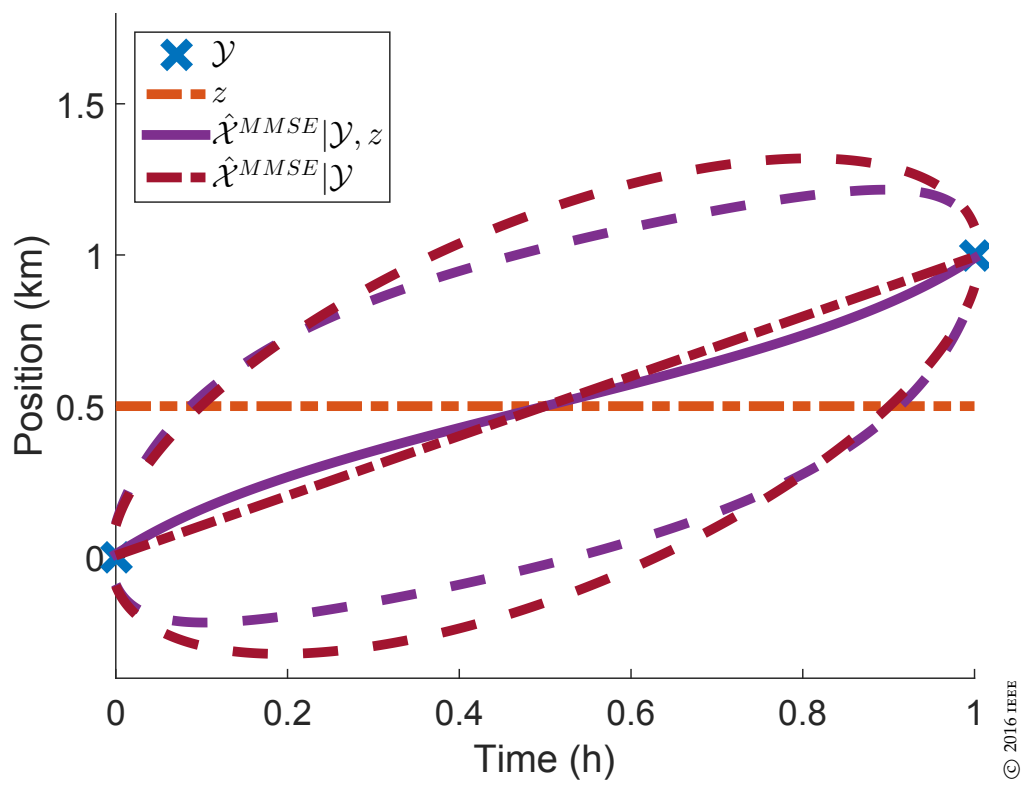

(a) First case.

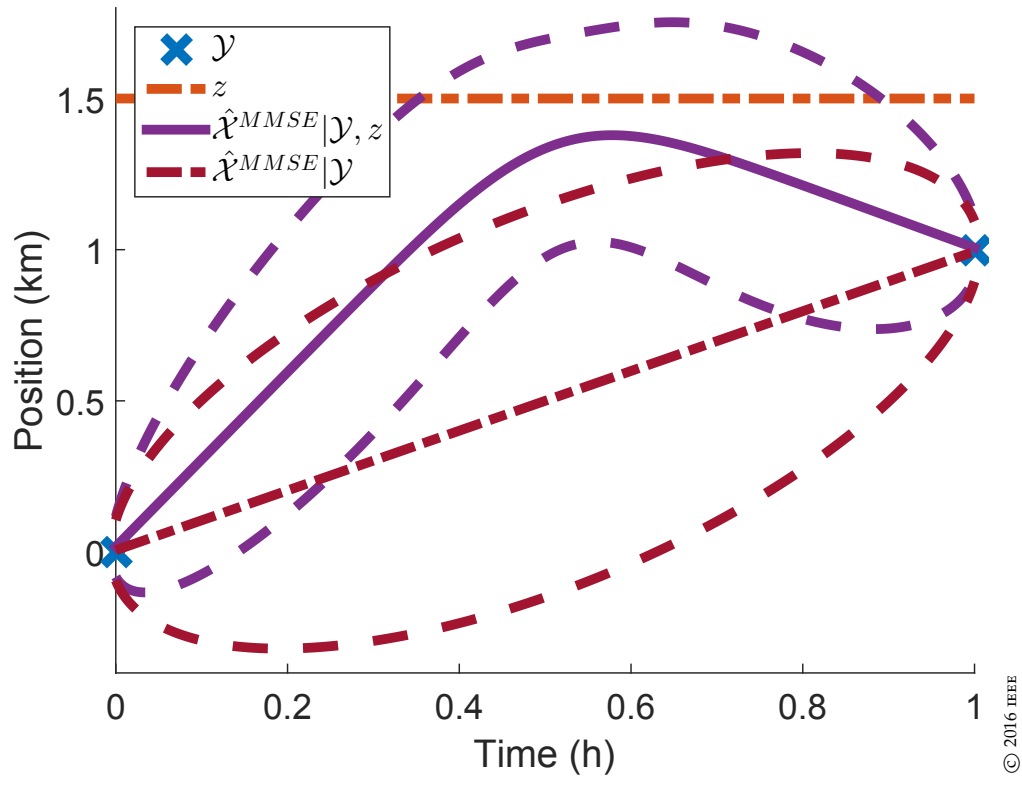

(b) Second case.

Figure 5.9: The MMSE estimate and its uncertainty compared to the MMSE estimate given only measurements for the two cases. Published in [182]. 


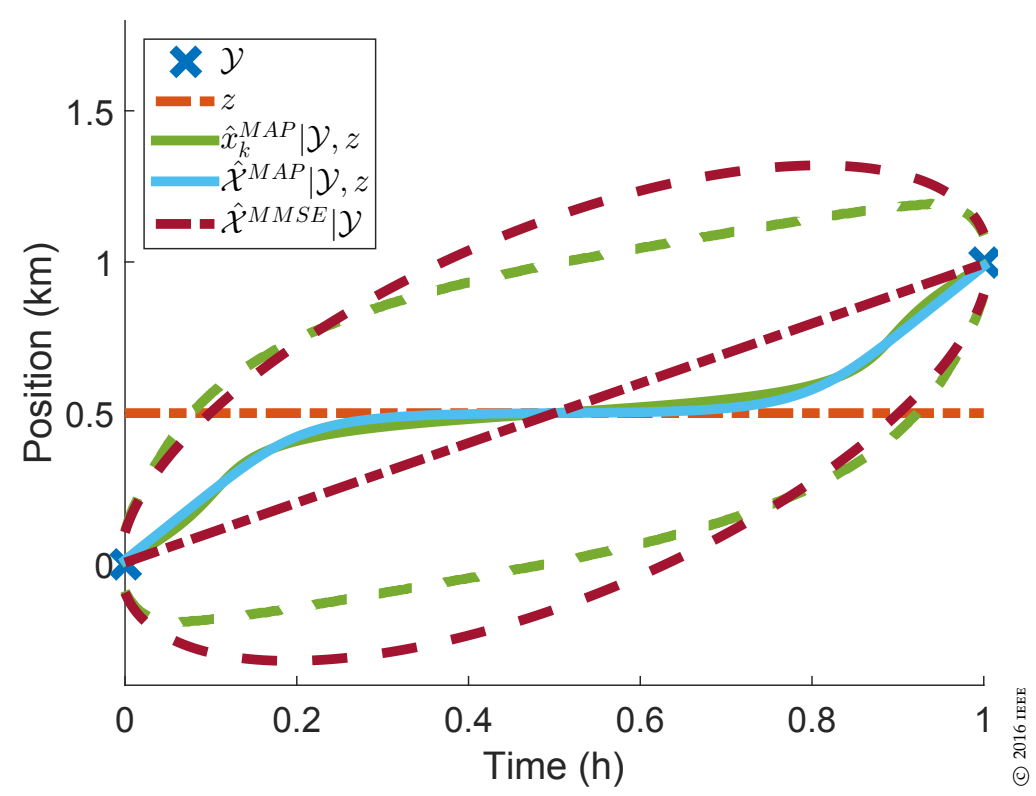

(a) First case.

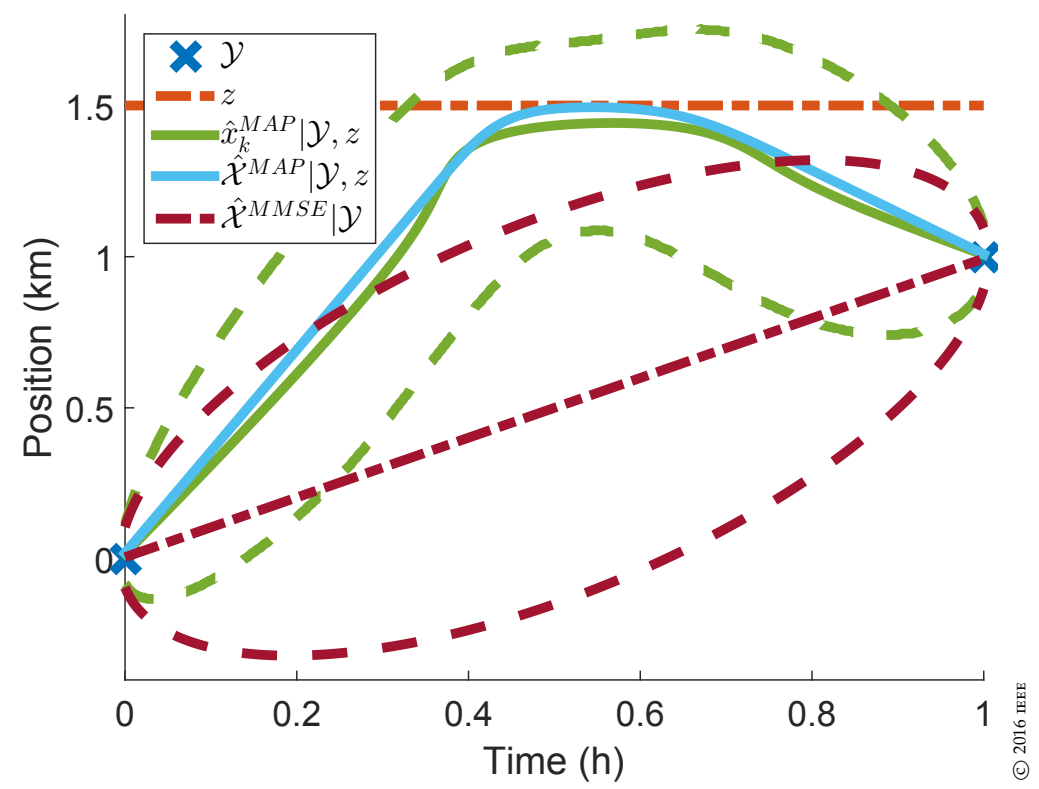

(b) Second case.

Figure 5.10: The MAP estimates of $p\left(\mathcal{X} \mid y_{1}, z_{1}\right)$ and $p\left(x_{k} \mid y_{1}, z_{1}\right)$ and the MMSE estimate given only the measurements for the two cases. Published in [182]. 
The MAP estimator for the orienteering application is presented in Application 5.3. The resulting trajectory of the sprinter when using the proposed model in this application is also compared to the original trajectory using only the GNSS measurements as well as the posterior distribution of the trajectory.

\section{Application 5.5: Orienteering Uncertain Time MAP Estimator}

The MAP estimate for the orienteering application is presented for a part of the map in Figure 5.11. The bias has been estimated and the trajectory of the sprinter has been adjusted to pass through the controls. Sample trajectories from the posterior distribution are also shown. The full trajectory is shown in Figure 5.12.

The improvement of the trajectory is limited for this particular set of data considering the GNSS measurement was rather accurate to begin with. However, the effects are present and it can be seen as a motivation for further development of the method. For more difficult terrain with large differences in altitude the GNSS is often less reliable. The practical use of the UTM on real data has been demonstrated. This concludes the orienteering application.

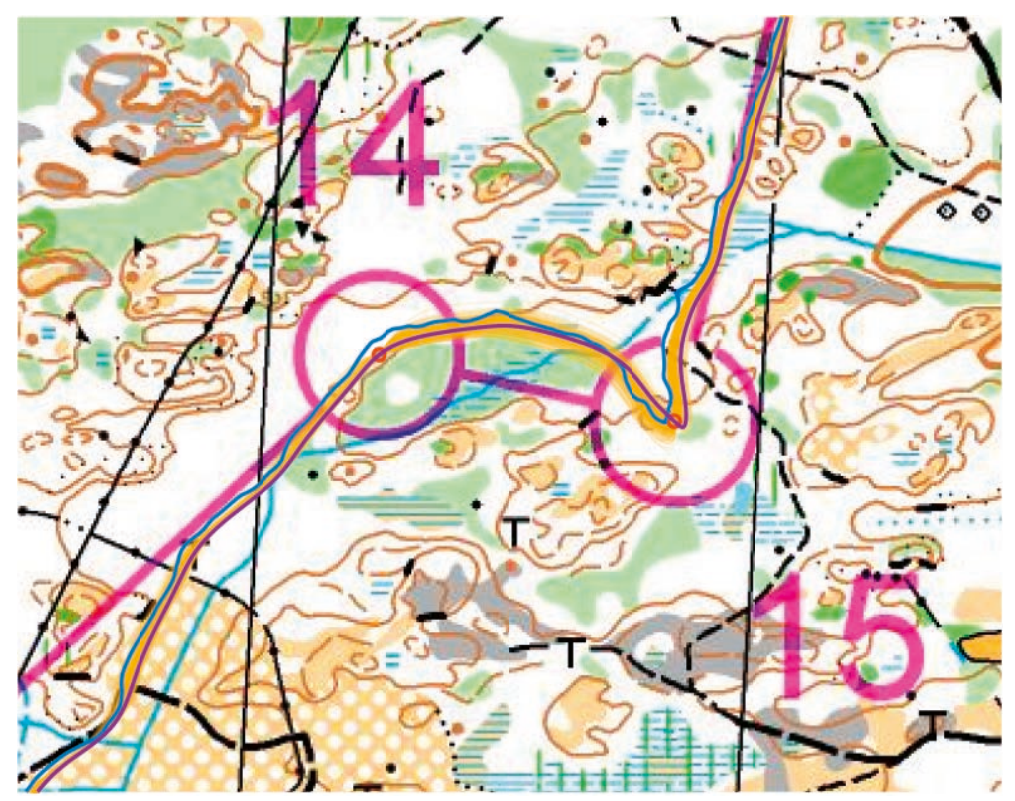

Figure 5.11: An enlargement around two controls of the results in the orienteering application. The MAP estimate (purple) and the posterior distribution (yellow) of the trajectory are compared to the GNSS measurements (blue) and the controls (red). Courtesy of OK Orion. 


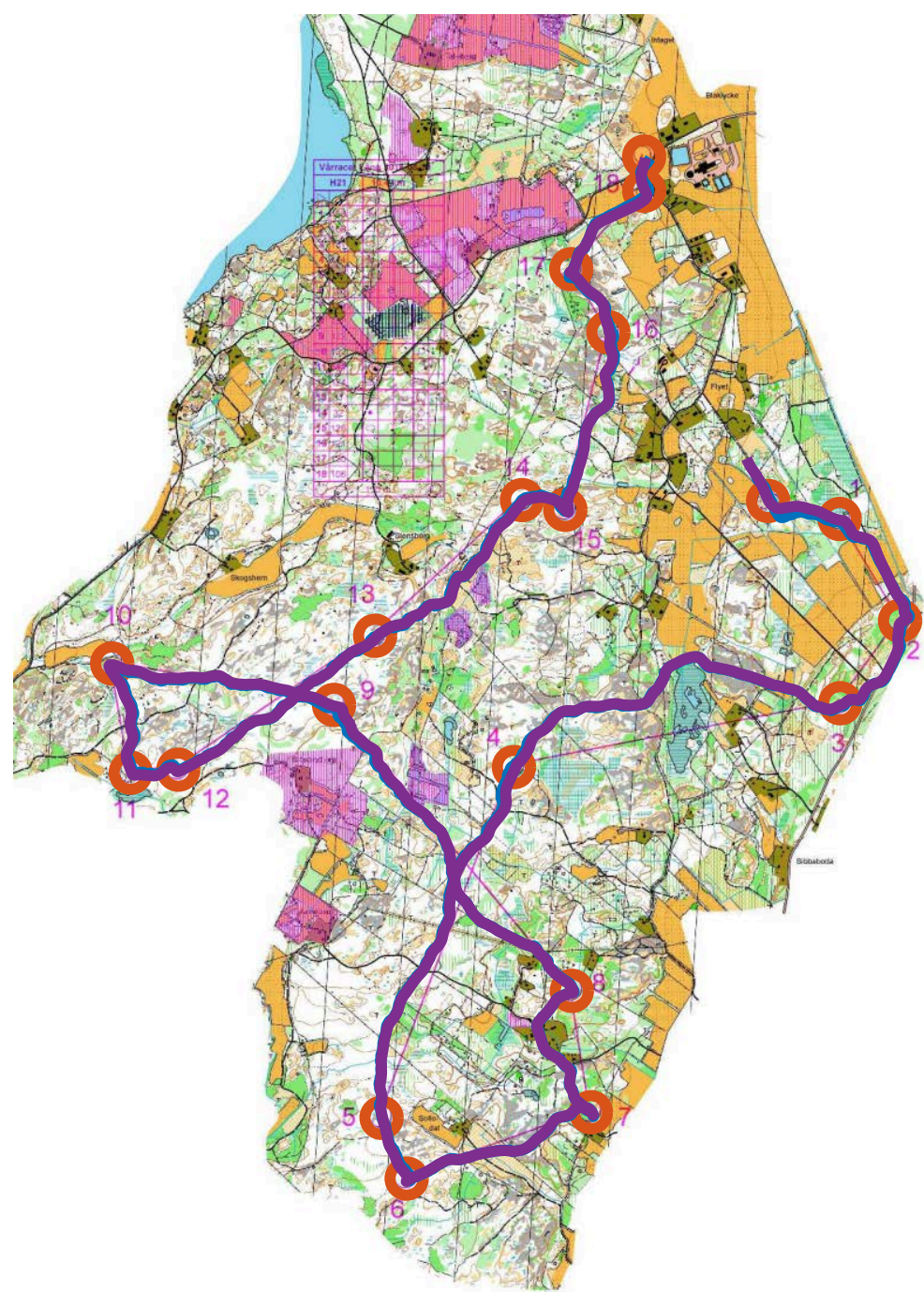

Figure 5.12: The MAP estimate (purple) for the orienteering application compared to the GNSS measurements (blue) and the controls (red). Courtesy of OK Orion. 


\section{Maximum A Posteriori Timestamp Estimator}

The MAP estimator [103] for the posterior distribution of the timestamps in (5.46) is given by

$$
\begin{aligned}
\hat{\mathcal{T}}^{\mathrm{MAP}}=\underset{\mathcal{T}}{\arg \max } p(\mathcal{T} \mid \mathcal{Y}, \mathcal{Z})=\underset{\mathcal{T}}{\arg \max } \log p(\mathcal{T} \mid \mathcal{Y}, \mathcal{Z})= \\
=\underset{\mathcal{T}}{\arg \max } \sum_{i \in \mathcal{I}} \log p\left(\tau_{i} \mid \mathcal{T}_{1: i-1}\right)+\log \mathcal{N}\left(\mathbf{z}_{i} \mid \hat{\mathbf{z}}_{i}^{\mathcal{T}_{1: i-1}}\left(\tau_{i}\right), \mathbf{S}_{i}^{\left.\mathcal{T}_{1: i-1}\left(\tau_{i}\right)\right)}\right. \\
=\underset{\mathcal{T}}{\arg \max } \sum_{i \in \mathcal{I}} 2 \log p\left(\tau_{i} \mid \mathcal{T}_{1: i-1}\right)-\log \left|\mathbf{S}_{i}^{\mathcal{T}_{1: i-1}}\left(\tau_{i}\right)\right| \\
\quad-\left\|\mathbf{z}_{i}-\hat{\mathbf{z}}_{i}^{\mathcal{T}_{1: i-1}}\left(\tau_{i}\right)\right\|_{\mathbf{s}_{i} \mathcal{T}_{1: i-1}\left(\tau_{i}\right)^{\prime}}^{2}
\end{aligned}
$$

using the monotonically increasing property of logarithms. Numerical optimization [138] can be used to obtain an estimate.

\section{Joint Maximum A Posteriori Estimator}

The JMAP estimator of the joint posterior distribution $p(\mathbf{x}(\cdot), \mathcal{T} \mid \mathcal{Y}, \mathcal{Z})$ is given by

$$
\begin{aligned}
\left\{\hat{\mathbf{x}}^{\mathrm{IMAP}}(\cdot), \hat{\mathcal{T}}^{\mathrm{JMAP}}\right\} & =\underset{\mathbf{x}(\cdot), \mathcal{T}}{\arg \max } p(\mathbf{x}(\cdot), \mathcal{T} \mid \mathcal{Y}, \mathcal{Z}) \\
& =\underset{\mathbf{x}(\cdot), \mathcal{T}}{\arg \max } p(\mathbf{x}(\cdot) \mid \mathcal{T}, \mathcal{Y}, \mathcal{Z}) p(\mathcal{T} \mid \mathcal{Y}, \mathcal{Z})
\end{aligned}
$$

which for the linear Gaussian model reduces to

$$
\left\{\hat{\mathbf{x}}^{\mathrm{JMAP}}(\cdot), \hat{\mathcal{T}}^{\mathrm{JMAP}}\right\}=\underset{\mathbf{x}(\cdot), \mathcal{T}}{\arg \max } w_{\mathcal{T}} \mathcal{N}\left(\mathbf{x}(\cdot) \mid \hat{\mathbf{x}}^{\mathcal{T}}(\cdot), \mathbf{P}^{\mathcal{T}}(\cdot)\right),
$$

where (5.45) and (5.46) are used. This can be rewritten as

$$
\begin{aligned}
\hat{\mathcal{T}}^{\text {JMAP }}= & \underset{\mathcal{T}}{\arg \max }\left\{w_{\mathcal{T}} \max _{\mathbf{x}(\cdot)} \mathcal{N}\left(\mathbf{x}(\cdot) \mid \hat{\mathbf{x}}^{\mathcal{T}}(\cdot), \mathbf{P}^{\mathcal{T}}(\cdot)\right)\right\} \\
= & \underset{\mathcal{T}}{\arg \max } \frac{w_{\mathcal{T}}}{\sqrt{\left|\mathbf{P}^{\mathcal{T}}(\cdot)\right|}} \\
= & \underset{\mathcal{T}}{\arg \max } \prod_{i \in \mathcal{I}} \sqrt{\left|\mathbf{R}_{i}^{z}+\mathbf{H}_{i}^{z} \mathbf{P}^{\mathcal{T}_{1: i-1}}\left(\tau_{i}\right)\left(\mathbf{H}_{i}^{z}\right)^{\top}\right|} \cdot \\
& p\left(\tau_{i} \mid \mathcal{T}_{1: i-1}\right) \cdot \mathcal{N}\left(\mathbf{z}_{i} \mid \hat{\mathbf{z}}_{i}^{\mathcal{T}_{1: i-1}}\left(\tau_{i}\right), \mathbf{s}_{i}^{\mathcal{T}_{1: i-1}}\left(\tau_{i}\right)\right) \\
= & \underset{\mathcal{T}}{\arg \max } \sum_{i \in \mathcal{I}} 2 \log p\left(\tau_{i} \mid \mathcal{T}_{1: i-1}\right)-\left\|\mathbf{z}_{i}-\hat{\mathbf{z}}_{i}^{\mathcal{T}_{1: i-1}}\left(\tau_{i}\right)\right\|_{\mathbf{s}_{i}}^{\mathcal{T}_{1: i-1}\left(\tau_{i}\right)^{\prime}}, \\
\hat{\mathbf{x}}^{\text {JMAP }}(\cdot)= & \hat{\mathbf{x}}^{\hat{\mathcal{T}}^{\text {JMAP }}}(\cdot),
\end{aligned}
$$

where Proposition 5.4 and (5.47) are used. Note that the function no longer depends on the joint covariance of all states, only the covariance of each state. 
Proposition 5.4: Given a joint posterior distribution $p(\mathbf{x}(\cdot) \mid \mathcal{Y})$ of an LGSSM described by (3.13), (3.62) and (3.63) with index $i$ dropped, where $\mathbf{P}(\cdot)=\operatorname{Cov}(\mathbf{x}(\cdot) \mid \mathcal{Y})$ and $\mathbf{P}(\cdot)$ and $\mathbf{R}^{z}$ are non-singular, the determinant of $\mathbf{P}^{\tau}(\cdot)=\operatorname{Cov}(\mathbf{x}(\cdot) \mid \mathcal{Y}, \mathbf{z}, \tau)$ is given by

$$
\left|\mathbf{P}^{\tau}(\cdot)\right|=|\mathbf{P}(\cdot)|\left|\mathbf{R}^{z}\right|\left|\mathbf{R}^{z}+\mathbf{H}^{z} \mathbf{P}(\tau)\left(\mathbf{H}^{z}\right)^{\top}\right|^{-1} .
$$

Proof: Let the observation be $\mathbf{z}=\mathbf{H}^{z} \mathbf{x}(\tau)+\mathbf{e}^{z}=\overline{\mathbf{H}}_{\tau} \mathbf{x}(\cdot)+\mathbf{e}^{z}$, then

$$
\begin{aligned}
\left|\mathbf{P}^{\tau}(\cdot)\right|^{-1} & =\left|\left[\mathbf{P}^{-1}(\cdot)+\left(\overline{\mathbf{H}}_{\tau}\right)^{\top}\left(\mathbf{R}^{z}\right)^{-1} \overline{\mathbf{H}}_{\tau}\right]^{-1}\right|^{-1} \\
& \left.=\mid \mathbf{P}^{-1}(\cdot)+\left(\overline{\mathbf{H}}_{\tau}\right)^{\top}\left(\mathbf{R}^{z}\right)^{-1} \overline{\mathbf{H}}_{\tau}\right) \mid \\
& =|\mathbf{P}(\cdot)|^{-1}\left|\mathbf{I}+\left(\mathbf{R}^{z}\right)^{-1} \overline{\mathbf{H}}_{\tau} \mathbf{P}(\cdot)\left(\overline{\mathbf{H}}_{\tau}\right)^{\top}\right| \\
& =|\mathbf{P}(\cdot)|^{-1}\left|\mathbf{R}^{z}\right|^{-1}\left|\mathbf{R}^{z}+\mathbf{H}^{z} \mathbf{P}(\tau)\left(\mathbf{H}^{z}\right)^{\top}\right|,
\end{aligned}
$$

where Millman's formula [2] was used in the first equality and Sylvester's identity [4] in the third equality, concluding the proof.

The JMAP estimator for the simple uncertain time scenario is presented in Application 5.3.

\section{Application 5.6: Simple Uncertain Time JMAP Estimator}

The JMAP state estimate $\hat{\mathcal{X}}^{\mathrm{JMAP}}$ is shown in Figure 5.13 for the two cases, together with the uncertainty of the state estimate given that the JMAP time estimate is correct. Since the uncertainty in time is disregarded, the uncertainty for the state estimate is underestimated. The estimated uncertain times $\hat{\tau}_{1}^{\mathrm{JMAP}}$ are 0.5 and 0.52 , respectively. See Application 5.7 for a comparison and analysis of the different estimators.

A comparison and an analysis of the various estimators for the simple uncertain time scenario are presented in Application 5.7.

\section{Application 5.7: Simple Uncertain Time Estimator Comparison}

The effects of the additional observation sampled at an uncertain time on the estimators are analysed. The analysis is based on the results presented in Applications 5.3, 5.4 and 5.6. Further, the posterior distribution of the timestamp presented in Application 5.2 is shortly discussed.

The effects of including an observation with an uncertain timestamp is most visible for the first case. Since the observation coincides with the previous trajectory, i.e., the estimate given only the measurements with precise timestamps, the JMAP estimator in Figure 5.13a will estimate $\tau_{1}$ at the time of coincidence and result in the same trajectory. The uncertainty is underestimated because it disregards the uncertainty in time.

The MMSE estimator in Figure 5.9a produces a trajectory that slightly tends towards the observation compared to the previous trajectory. The reason for this behaviour can be found in Figure 5.8a where most weights, given by $p\left(\tau_{1} \mid y_{1}, z_{1}\right)$, are similar except near the measurements with precise timestamps. The trajectory for each weight passes near the observation at its corresponding time, attracting the weighted average of the trajectories in the MMSE estimate towards it. 


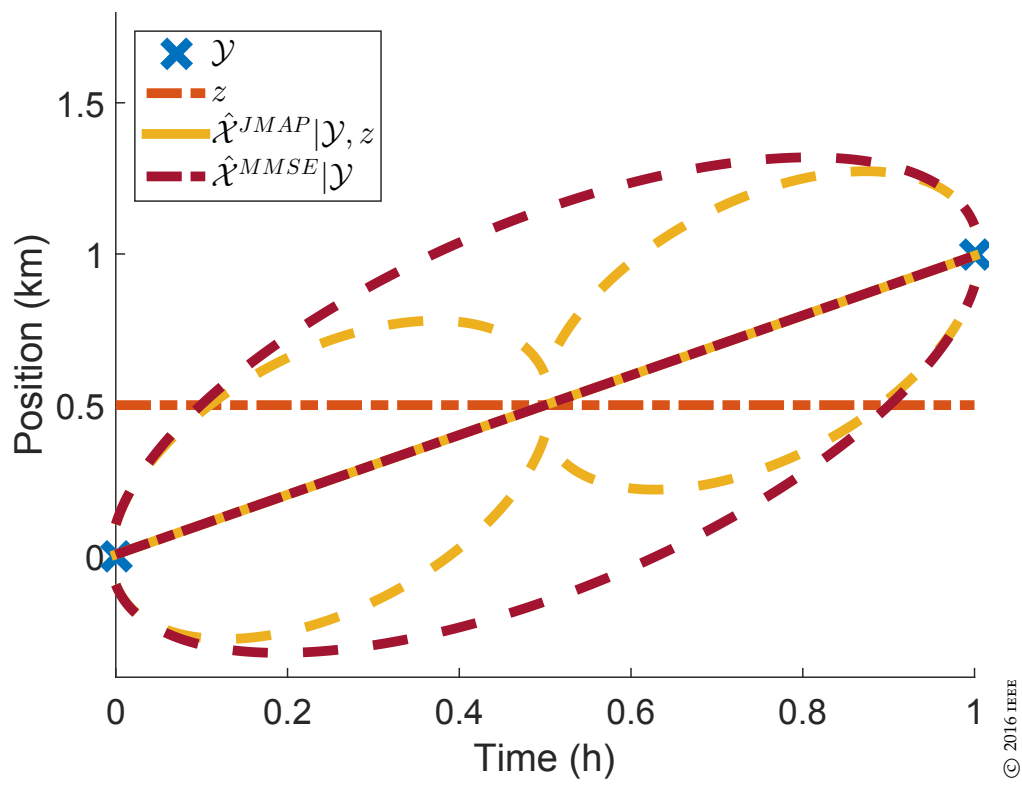

(a) First case.

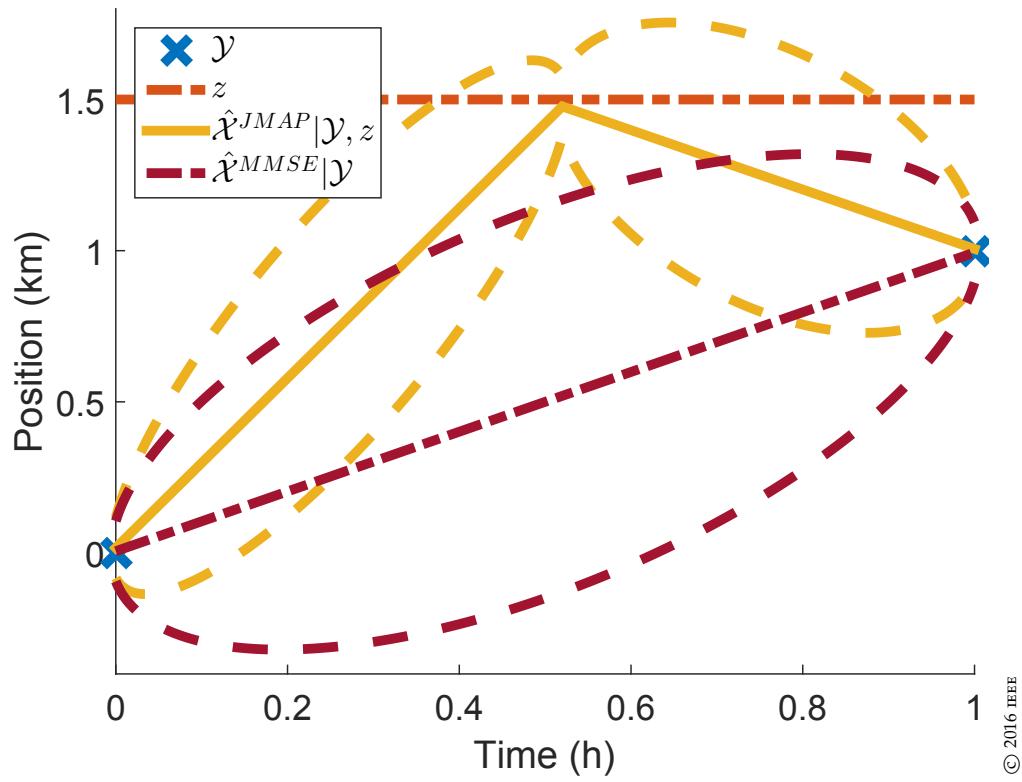

(b) Second case.

Figure 5.13: The JMAP state estimate of $p\left(\mathcal{X}, \tau_{1} \mid y_{1}, z_{1}\right)$ and the MMSE estimate given only the measurements for the two cases. Published in [182]. 
It should also be noted that the uncertainty for the estimate is reduced compared to the previous trajectory.

The behaviour of tending towards the observation is even more pronounced for the MAP estimators in Figure 5.10a. For the MAP estimator of $p\left(x_{k} \mid y_{1}, z_{1}\right)$, this can intuitively be understood by realizing that at each time within the region of similar weights in Figure 5.8a the heights of the peaks will be approximately proportional to the inverse of the covariance, and the smallest covariance is given by the Gaussian where the observation was used to update the posterior distribution at the current time. This will attract the trajectory even more towards the observation than the MMSE estimate. Although harder to visualize, due to the high dimension, a similar intuition applies to the MAP estimator for $p\left(\mathcal{X} \mid y_{1}, z_{1}\right)$. The MAP estimate of the joint smoothing distribution $p\left(\mathcal{X} \mid y_{1}, z_{1}\right)$ is also smoother than the MAP estimates of the marginal smoothing distribution $p\left(x_{k} \mid y_{1}, z_{1}\right)$, which can be explained by the discarded cross-covariances in the latter. The uncertainty of $p\left(x_{k} \mid y_{1}, z\right)$ is also reduced significantly over the previous trajectory.

For the second case the estimates are drastically different since the observation does not coincide with the previous trajectory. The other difference compared to the first case is that the prior distribution $p\left(\tau_{1}\right)$ is no longer flat, see Figure 5.8b. This gives much more influence to trajectories corresponding to the peaked region. The JMAP estimator in Figure 5.13b produces the trajectory that corresponds to the maximum of $p\left(\mathcal{X}, \tau_{1} \mid y_{1}, z_{1}\right)$. As before, the MMSE estimate in Figure $5.9 \mathrm{~b}$ is a weighted average of trajectories, thus not reaching all the way to the observation. The MAP estimates $p\left(x_{k} \mid y_{1}, z_{1}\right)$ and $p\left(\mathcal{X} \mid y_{1}, z_{1}\right)$ tend even more to the observation, which can be explained with similar intuition as for the first case.

The derivations for the MAP and JMAP estimators allows for further analysis of the posterior distribution of the timestamp in Figure 5.8a. As can be seen in (5.55a) $\max _{\mathcal{X}} p\left(\mathcal{X}, \tau_{1} \mid y_{1}, z_{1}\right)$ depends on the prior distribution, which is constant in this case, as well as the Mahalanobis distance between the observation and its prediction. In (5.52), however, it can be seen that $p\left(\tau_{1} \mid y_{1}, z_{1}\right)$ in addition depends on the determinant of the innovation covariance, in effect penalizing uncertainty in the likelihood of $z_{1}$. By considering $\max _{\mathcal{X}} p\left(\mathcal{X}, \tau_{1} \mid y_{1}, z_{1}\right)$, the reduction of uncertainty in the joint posterior distribution is also taken into account, while only the likelihood of the observation given the measurements is considered in $p\left(\tau_{1} \mid y_{1}, z_{1}\right)$.

An analysis of the implications of an uncertain timestamp on estimation in a simple one-dimensional scenario has been performed. This concludes the simple uncertain time scenario.

\subsubsection{Approximate Computational Methods}

The timestamps of the UTM in (3.63) are assumed to be continuous. This results in difficulties in optimizing and computing the integrals in (5.48)-(5.52) analytically. Three methods for approximately computing the posterior distributions 
and point estimators are presented. Due to the discretization of the timestamp prior, the first method is implicitly used for the results of the simple uncertain time application presented in the previous section.

\section{Discrete Timestamp Approximation}

One method is to approximate the prior on the timestamps in (3.63) as a discrete distribution in the time instants $\mathcal{T}_{z}$. The posterior distribution then reduces from a compound distribution to a mixture distribution. This is effectively equivalent to replacing the integrals with summations in (5.48)-(5.51). With high enough resolution in time, the effect on the results is negligible, however, the choice is a trade-off between accuracy and computation time.

For the UTM the resulting posterior is a mixture of Gaussians. The MMSE estimator for this distribution is straightforward to compute. However, the MAP estimator still requires numerical optimization methods [138].

This method was used in [182] when only a single observation of type o was considered. However, this option is not viable as the only approximation when multiple observations are considered since the number of components in the mixture grows exponentially with the number of type o observations.

\section{Gibbs Sampling}

Another method is to approximate the distribution in (5.48) by sampling. To compute the posterior distribution in (5.48), one needs to integrate over an I-dimensional space. Even if the space is discretized, as discussed in the previous section, it is in general too costly to exhaustively explore the space of multiple observations when computing the distribution. As an alternative the distribution can be approximated using Gibbs sampling [69, 71]. The timestamps are sampled from the distribution $p(\mathcal{T} \mid \mathcal{Y}, \mathcal{Z})$ given in (5.46). The posterior distribution of the states conditional on the timestamps is computed using the methods discussed in Section 5.3.

In the Gibbs sampler, samples of the timestamps are drawn iteratively conditional on the current samples of all other timestamps. The sample $\tau_{i}^{s}$ of the timestamp for observation $\mathbf{z}_{i}$ in iteration $s$ is drawn from the distribution

$$
\begin{aligned}
p\left(\tau_{i} \mid \mathcal{T}_{\neg i}^{s}, \mathcal{Y}, \mathcal{Z}\right) & \propto p\left(\mathbf{z}_{i} \mid \mathcal{Y}, \mathcal{Z}_{\neg i}, \mathcal{T}_{\neg i}^{s}, \tau_{i}\right) \cdot p\left(\tau_{i} \mid \mathcal{T}_{\neg i}^{s}\right) \\
& =\mathcal{N}\left(\mathbf{z}_{i} \mid \mathbf{H}_{i}^{z} \hat{\mathbf{x}}_{\neg i}^{\mathcal{T}}\left(\tau_{i}\right), \mathbf{H}_{i}^{z} \mathbf{P}_{\neg i}^{\mathcal{T}_{\neg i}^{s}}\left(\tau_{i}\right)\left(\mathbf{H}_{i}^{z}\right)^{\top}+\mathbf{R}_{i}^{z}\right) \cdot p\left(\tau_{i} \mid \mathcal{T}_{\neg i}^{s}\right) \\
& =\mathcal{N}\left(\mathbf{z}_{i} \mid \hat{\mathbf{z}}_{i}^{\mathcal{\mathcal { T } ^ { s }}}\left(\tau_{i}\right), \mathbf{S}_{i}^{\mathcal{T}_{\neg i}^{s}}\left(\tau_{i}\right)\right) \cdot p\left(\tau_{i} \mid \mathcal{T}_{\neg i}^{s}\right),
\end{aligned}
$$

where $\mathcal{Z}_{\neg i}=\mathcal{Z} \backslash \mathbf{z}_{i}, \mathcal{T}_{\neg i}^{s}=\mathcal{T}_{1: i-1}^{s} \cup \mathcal{T}_{i+1: I}^{s-1}$ and similar notation as in (5.47) is used in the last equality.

In the case that the timestamps are discretized, this distribution is simple to sample from, since it is gridded in one-dimension. However, for continuous timestamps rejection sampling [70], adaptive rejection Metropolis sampling [73], Metropolis sampling [126] or sampling/importance resampling [156, 163] can be used within the Gibbs sampler. 
A set $\left\{\mathcal{T}^{s}\right\}_{S=1}^{S}$ of timestamp samples is obtained to discretely approximate the distribution $p(\mathcal{T} \mid \mathcal{Y}, \mathcal{Z})$ in (5.46). The integral in (5.48) reduces to a sum over the samples and approximates the posterior distribution as

$$
p(\mathbf{x}(\cdot) \mid \mathcal{Y}, \mathcal{Z}) \approx \frac{1}{S} \sum_{s=1}^{S} \mathcal{N}\left(\mathbf{x}(\cdot) \mid \hat{\mathbf{x}}^{\mathcal{T}^{s}}(\cdot), \mathbf{P}^{\mathcal{T}^{s}}(\cdot)\right)
$$

The MMSE estimator presented in Section 5.6.2 is similarly approximated using this discrete set of timestamps as

$$
\hat{\mathbf{x}}^{\mathrm{MMSE}}(\cdot) \approx \frac{1}{S} \sum_{S=1}^{S} \hat{\mathbf{x}}^{\mathcal{T}_{s}}(\cdot)
$$

\section{Expectation-Maximization of the State}

An approximate method that can be employed to compute the MAP of the states in (5.51) is a combination of the discrete timestamp approximation and the expectation-maximization (EM) method [53]. The EM algorithm is an efficient method to find MAP estimates of parameters when the model includes unobserved latent variables. Although the method guarantees convergence to a mode, unfortunately there is no guarantee for convergence to the MAP estimate of the posterior distribution. This can be mitigated by using multiple starting points of the algorithm. A similar approach was presented in [45]. Interestingly, the method reduces to iteratively computing posterior distributions for LGSSMS.

The EM method iterates between computing an expectation over the timestamps and maximizing the expression with regards to the state. The expectation over the timestamps given the previous trajectory $\mathbf{x}^{-}(\cdot)$ is computed as

$$
\begin{aligned}
& R\left(\mathbf{x}(\cdot), \mathbf{x}^{-}(\cdot)\right)=\mathrm{E}_{\mathcal{T} \mid \mathcal{Z}, \mathcal{Y}, \mathbf{x}^{-}(\cdot)}[\log (p(\mathcal{T}, \mathbf{x}(\cdot), \mathcal{Z}, \mathcal{Y}))] \\
& =\int p\left(\mathcal{T} \mid \mathcal{Z}, \mathbf{x}^{-}(\cdot)\right)[\log p(\mathcal{Z} \mid \mathbf{x}(\cdot), \mathcal{T})+ \\
& \quad \log p(\mathbf{x}(\cdot) \mid \mathcal{Y})+\log p(\mathcal{T})+\log p(\mathcal{Y})] d \mathcal{T} \\
& =C+\log p(\mathbf{x}(\cdot) \mid \mathcal{Y})+\sum_{i \in \mathcal{I}} \int w_{i}\left(\tau_{i}\right) \log \mathcal{N}\left(\mathbf{z}_{i} \mid \mathbf{H}_{i}^{z} \mathbf{x}\left(\tau_{i}\right), \mathbf{R}_{i}^{z}\right) d \tau_{i}
\end{aligned}
$$

where $C$ is a constant that is independent of $\mathbf{x}(\cdot)$ and

$$
w_{i}\left(\tau_{i}\right)=\int p\left(\mathcal{T} \mid \mathcal{Z}, \mathbf{x}^{-}(\cdot)\right) d \mathcal{T}_{\neg i}=p\left(\tau_{i} \mid \mathcal{Z}, \mathbf{x}^{-}(\cdot)\right),
$$

using the notation $\mathcal{T}_{\neg i}=\mathcal{T} \backslash \tau_{i}$. This is the marginal distribution of $\tau_{i}$ given all observations and the state. For independent priors on the timestamps the expression simplifies to

$$
w_{i}\left(\tau_{i}\right)=\frac{\mathcal{N}\left(\mathbf{z}_{i} \mid \mathbf{H}_{i}^{z} \mathbf{x}^{-}\left(\tau_{i}\right), \mathbf{R}_{i}^{z}\right) p\left(\tau_{i}\right)}{\int \mathcal{N}\left(\mathbf{z}_{i} \mid \mathbf{H}_{i}^{z} \mathbf{x}^{-}(\tau), \mathbf{R}_{i}^{z}\right) p\left(\tau_{i}=\tau\right) d \tau} .
$$


For dependent priors on the timestamps, the joint distribution in the integrand of (5.62) can be sampled, e.g. using a Gibbs sampler [71], and marginalized to obtain samples from $w_{i}\left(\tau_{i}\right)$. Using $\tilde{\mathbf{R}}_{i}^{z}(\tau)=w_{i}^{-1}(\tau) \mathbf{R}_{i}^{z}$ the expectation in (5.61) reduces to

$$
R\left(\mathbf{x}(\cdot), \mathbf{x}^{-}(\cdot)\right)=\tilde{C}+\log p(\mathbf{x}(\cdot) \mid \mathcal{Y})-\frac{1}{2} \int \sum_{i \in \mathcal{I}}\left\|\mathbf{z}_{i}-\mathbf{H}_{i}^{z} \mathbf{x}(\tau)\right\|_{\tilde{\mathbf{R}}_{i}^{z}(\tau)}^{2} d \tau .
$$

If the observation matrix is the same for all observations, such that $\mathbf{H}_{i}^{z}=\mathbf{H}^{z}$, the expectation in (5.61) further reduces to

$$
R\left(\mathbf{x}(\cdot), \mathbf{x}^{-}(\cdot)\right)=\hat{C}+\log p(\mathbf{x}(\cdot) \mid \mathcal{Y})-\frac{1}{2} \int\left\|\overline{\mathbf{z}}(\tau)-\mathbf{H}^{z} \mathbf{x}(\tau)\right\|_{\overline{\mathbf{R}}^{z}(\tau)}^{2} d \tau,
$$

where an equivalent observation is used, given by

$$
\begin{aligned}
\overline{\mathbf{R}}^{z}(\tau) & =\left[\sum_{i \in \mathcal{I}} w_{i}(\tau)\left(\mathbf{R}_{i}^{z}\right)^{-1}\right]^{-1}, \\
\overline{\mathbf{z}}(\tau) & =\overline{\mathbf{R}}^{z}(\tau)\left[\sum_{i \in \mathcal{I}} w_{i}(\tau)\left(\mathbf{R}_{i}^{z}\right)^{-1} \mathbf{z}_{i}\right] .
\end{aligned}
$$

The trajectory maximizing (5.61) is obtained in the maximization step as

$$
\mathbf{x}^{+}(\cdot)=\underset{\mathbf{x}(\cdot)}{\arg \max } R\left(\mathbf{x}(\cdot), \mathbf{x}^{-}(\cdot)\right)
$$

where the + denotes the trajectory to use in the next iteration.

The expectation expression involves integrals over continuous time, which requires a continuous-time state trajectory to be estimated in each iteration. In practice, however, the state trajectory cannot be estimated in continuous time. One option for implementation is to discretize the prior [45] according to the discussion earlier in this section. This reduces the integrals in (5.61) and (5.63) to summations and allows the state to be considered in the discrete times $\mathcal{T}_{x}=$ $t_{0} \cup \mathcal{T}_{p} \cup \mathcal{T}_{y} \cup \mathcal{T}_{z}$

Let $\mathcal{X}$ be a column vector of the states at the time instants in $\mathcal{T}_{x}$, then (5.61) becomes

$$
\begin{aligned}
R\left(\mathcal{X}, \mathcal{X}^{-}\right)=\tilde{C} & +\log p(\mathcal{X} \mid \mathcal{Y})-\frac{1}{2} \sum_{i \in \mathcal{I}} \sum_{\tau_{i} \in \mathcal{T}_{z}}\left\|\mathbf{z}_{i}-\mathbf{H}_{i}^{z} \mathbf{x}\left(\tau_{i}\right)\right\|_{\tilde{\mathbf{R}}_{i}^{z}\left(\tau_{i}\right)}^{2} \\
=\bar{C} & -\frac{1}{2} \sum_{j \in \mathcal{J}}\left\|\mathbf{y}_{j}-\mathbf{H}_{j}^{y} \mathbf{x}\left(t_{j}^{y}\right)\right\|_{\tilde{\mathbf{R}}_{j}^{y}}^{2}-\frac{1}{2}\left\|\mathbf{x}\left(t_{0}\right)-\overline{\mathbf{x}}_{0}\right\|_{\mathbf{P}_{0}}^{2} \\
& -\frac{1}{2} \sum_{k \in \mathcal{K}}\left\|\mathbf{x}\left(t_{k}\right)-\mathbf{F}_{k} \mathbf{x}\left(t_{k-1}\right)\right\|_{\mathbf{Q}_{k}}^{2} \\
& -\frac{1}{2} \sum_{\tau \in \mathcal{T}_{z}} \sum_{i \in \mathcal{I}}\left\|\mathbf{z}_{i}-\mathbf{H}_{i}^{z} \mathbf{x}(\tau)\right\|_{\tilde{\mathbf{R}}_{i}^{z}(\tau)}^{2} .
\end{aligned}
$$


The trajectory $\mathcal{X}^{+}$maximizing this expression can be obtained as discussed in Section 5.3, noting that this problem can be reformulated as an equivalent LGSSM by replacing the observations in the UTM with the pseudo-observations

$$
\mathbf{z}_{i}=\mathbf{H}_{i}^{z} \mathbf{x}(\tau)+\tilde{\mathbf{e}}_{i}^{z}(\tau), \quad i \in \mathcal{I}, \tau \in \mathcal{T}_{z},
$$

where $\tilde{\mathbf{e}}_{i}^{z}(\tau) \sim \mathcal{N}\left(\mathbf{0}, \tilde{\mathbf{R}}_{i}^{z}(\tau)\right)$. The methods proposed for estimation are applied to the simulated uncertain time scenario in Application 5.8.

\section{Application 5.8: Simulated Uncertain Time Estimation}

Using sampling importance resampling within a Gibbs sampler, as described in this section, with 100 and 200 samples respectively, the posterior distribution of the timestamps is sampled as shown in Figure 5.15. For each sampled timestamp, a sample is drawn from the conditional posterior distribution to obtain samples from the posterior distribution of the states. The sampled states are shown in Figure 5.14. The MMSE estimates of the distributions are shown as well as the MAP estimates. The latter is obtained by discretizing the prior in 200 points and applying the EM algorithm. For the dependent prior an additional Gibbs sampler with 300 samples was used inside the EM algorithm to approximate the timestamp distribution given a trajectory and the observations.

To compare the estimators the root mean squared error (RMSE) averaged over the time instants in $\mathcal{T}_{p}$ and 500 Monte Carlo simulations is computed. The data is generated as described in Application 3.8 and the estimators use the model in (3.65). To obtain an upper and lower bound, the RMSE when discarding the observations $\mathcal{Z}$ and knowing the true timestamps $\mathcal{T}$ are computed. Three different priors are considered, the true prior used to generate the timestamps, the ordering prior and the uniform prior. For each prior the MMSE and MAP estimators are computed, as well as the JMAP estimate of the states and timestamps. The results for position and velocity estimates are presented in Table 5.2.

The results presented in Application 5.8 are further discussed in Application 5.9 .

\section{Application 5.9: Simulated Uncertain Time Discussion}

The sampled trajectories in Figure 5.14 are rather dense near the measurements, but more spread-out in the gaps where the uncertainty is large. One can see in Figure 5.15a that with the uniform prior, the sampled timestamps form a few clusters at the peaks of the multimodal posterior distribution. In Figure 5.15b one can see that infeasible timestamps are disregarded with the ordering prior, and almost all timestamps are sampled in one cluster around the highest peak.

An advantage of the MMSE estimator is that it is unbiased [103]. However for multimodal distributions a disadvantage is that the estimate might fall between modes, producing an unlikely estimate, as can be seen in Figure 5.14a. The posterior distribution in Figure 5.15b, however, is almost unimodal resulting in a MMSE estimate in Figure 5.14b that is more feasible.

The MAP estimator will on the other hand produce likely estimates of the state, but at the cost of being biased [103]. The MAP estimators in Figure 5.14 


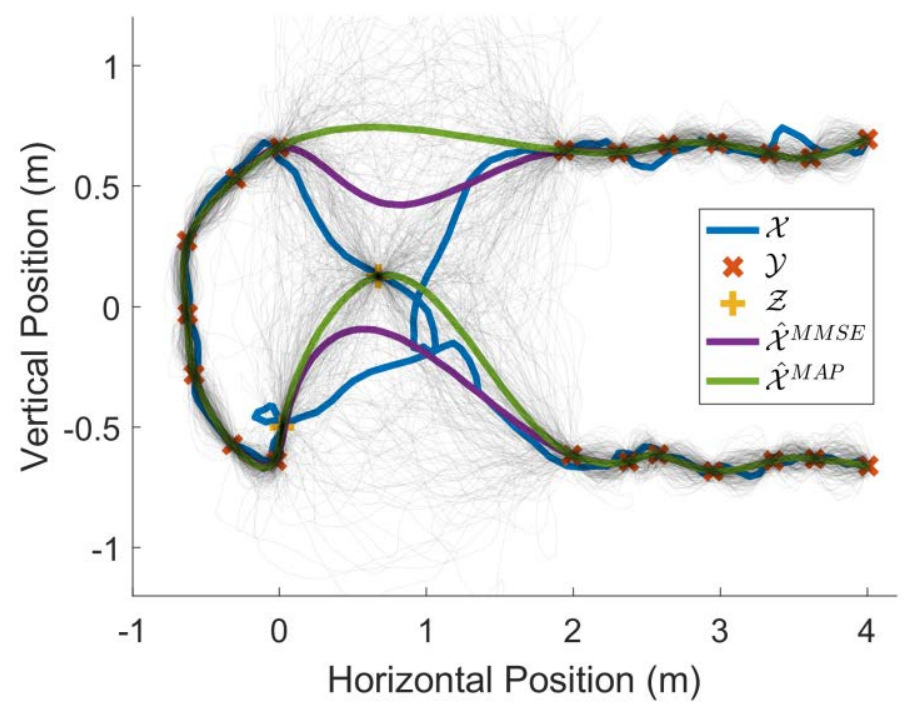

(a) Uniform prior.

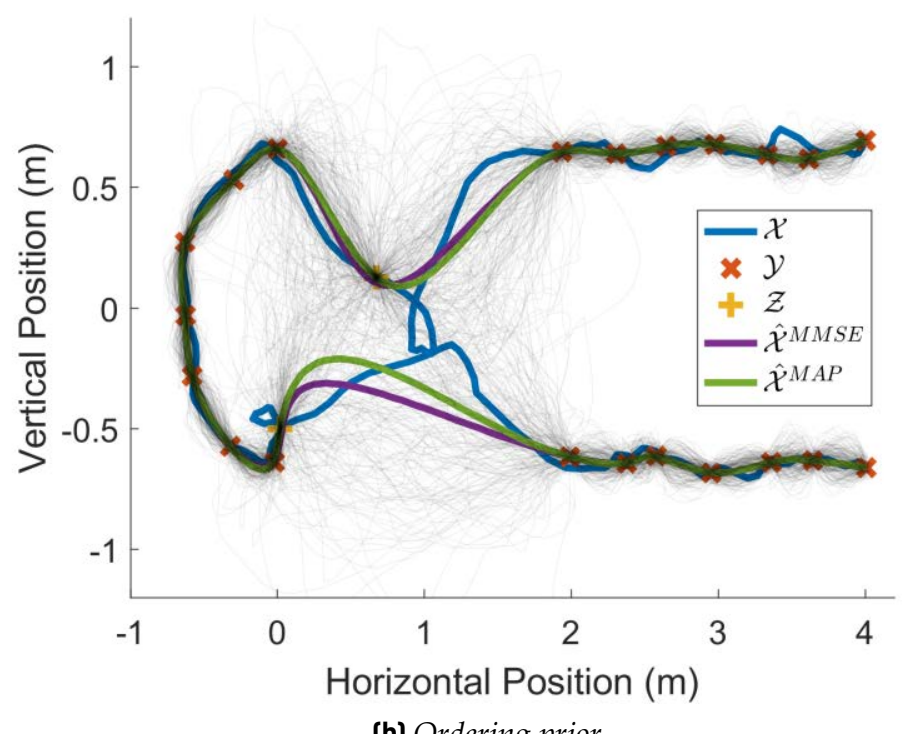

(b) Ordering prior.

Figure 5.14: The realization and estimators for the two-dimensional scenario, with samples from the posterior distribution (grey). The upper right measurement and topmost observation correspond to the first of their type. Published in [183]. 


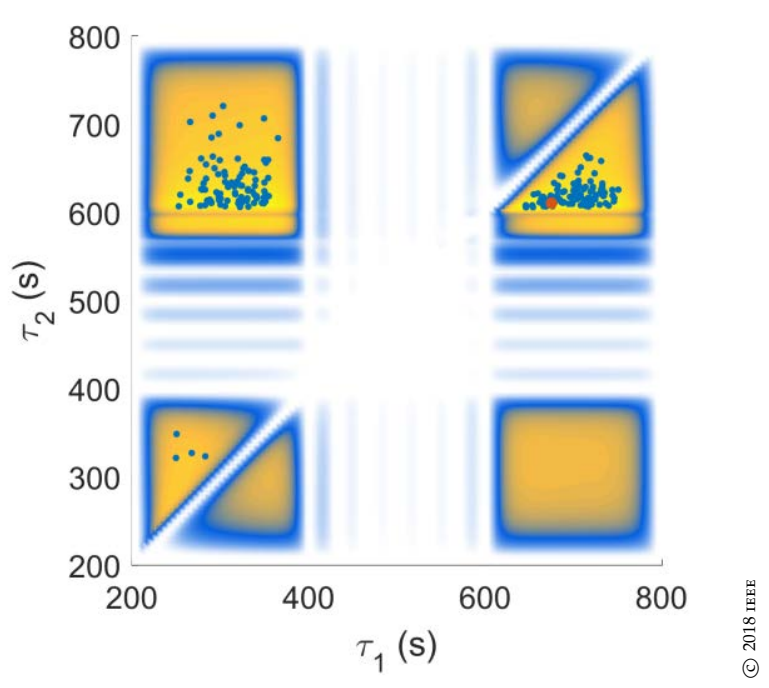

(a) Uniform prior.

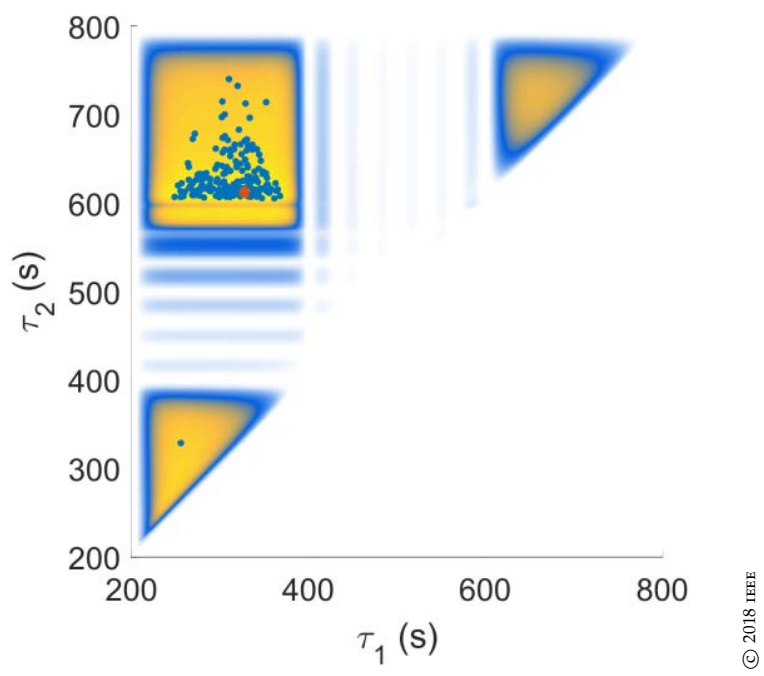

(b) Ordering prior.

Figure 5.15: The posterior distribution of the timestamps for the two-dimensional scenario. White and blue indicate low probability and yellow indicates medium to high probability. Samples from the distribution are shown as blue dots and the MAP estimate is shown in red. The bottom right triangle in (b) has exactly zero probability due to the ordering prior. Published in [183]. 
Table 5.2: RMSE of position and velocity averaged over time and 500 Monte Carlo simulations for various estimators. The percentage indicates the RMSE relative to the two reference estimators.

\begin{tabular}{rcccc}
\hline Estimator & \multicolumn{2}{c}{ Position $(\mathrm{m})$} & \multicolumn{2}{c}{ Velocity $(\mathrm{m} / \mathrm{s})$} \\
\hline Discarding $\mathcal{Z}$ & 0.2319 & $100.00 \%$ & 0.0066 & $100.00 \%$ \\
True $\mathcal{T}$ & 0.1562 & $0.00 \%$ & 0.0059 & $0.00 \%$ \\
MMSE True prior & 0.1813 & $33.16 \%$ & 0.0061 & $28.57 \%$ \\
MAP True prior & 0.1931 & $48.75 \%$ & 0.0062 & $42.86 \%$ \\
JMAP True prior & 0.1932 & $48.88 \%$ & 0.0062 & $42.86 \%$ \\
MMSE Ordering prior & 0.1863 & $39.76 \%$ & 0.0062 & $42.86 \%$ \\
MAP Ordering prior & 0.1939 & $49.80 \%$ & 0.0063 & $57.14 \%$ \\
JMAP Ordering prior & 0.2008 & $58.92 \%$ & 0.0063 & $57.14 \%$ \\
MMSE Uniform prior & 0.1885 & $42.67 \%$ & 0.0062 & $42.86 \%$ \\
MAP Uniform prior & 0.2012 & $59.45 \%$ & 0.0063 & $57.14 \%$ \\
JMAP Uniform prior & 0.2064 & $66.31 \%$ & 0.0064 & $71.43 \%$ \\
\hline
\end{tabular}

result in likely trajectories that visit the observations, although the estimated order might be incorrect as in Figure 5.14a and 5.15a if the prior is not sufficiently informative. One disadvantage with the MAP estimator is that other likely trajectories corresponding to other modes in Figure 5.15 are completely disregarded. By using multiple initializations other modes may be obtained, which might be informative in presentation.

The bottom left and top right clusters in Figure 5.15a represent that both observations are visited either on the way out or back, where the timestamp MAP estimate belongs to the latter. Note that the order of the timestamps for all samples in each cluster are the same. This is reasonable due to backtracking being unlikely. The top left cluster represents that the closest observation is visited on the way out and coming back, which represents the distribution that the timestamps were generated from. The infeasible timestamps using the ordering prior are rejected as expected in Figure 5.15b, resulting in a timestamp MAP estimate that belongs to the correct cluster.

For all three priors, the MMSE estimator outperforms the other estimators both for position and velocity. This is reasonable considering the purpose of the estimator is to minimize squared errors. The MAP estimator performs worse, but has the advantage of producing a likely estimate, which is not guaranteed for the MMSE estimator. The poor performance of the JMAP estimator can be explained by the lack of marginalization over the timestamps, which is inherent for the two other estimators. It is also seen that the performance improves with the amount of information put into the prior, but the improvement is relatively small compared to the bounds or to the difference between the estimators.

It should be noted that the particular scenario is designed to show the advantage of the additional observations. Nonetheless, it is shown that the performance improvement can be significant in such situations. This concludes the simulated uncertain time scenario. 


\subsection{Gaussian Process Motion Model Filter}

The posterior smoothing distribution for the states is derived for the model in Section 3.9.1. For the remaining linear models recursive solutions are obtained using KFS [101], presented in Section 5.2.1. The posterior distribution for the last model is approximated using an EKF [164]. See Section 3.9.1 for a description of the notation used for the covariance matrices.

\subsubsection{Posterior Smoothing Distribution}

The model in Section 3.9.1 is linear and Gaussian and its posterior can be derived analytically. Let $\mathcal{X}=\left(\mathbf{x}_{0}^{\top}, \ldots, \mathbf{x}_{K}^{\top}\right)^{\top}, \mathcal{F}=\left(\mathbf{f}_{1}^{\top}, \ldots, \mathbf{f}_{K}^{\top}\right)^{\top}$ and $\mathcal{Y}=\left(\mathbf{y}_{1}^{\top}, \ldots, \mathbf{y}_{K}^{\top}\right)^{\top}$. Replacing (3.74d)-(3.74e) with $\mathcal{F} \sim \mathcal{N}\left(\mathbf{0}, \tilde{\mathbf{K}}_{f f}\right)$ and moving knowns to the left and unknowns to the right allows a representation of (3.74) on the form

$$
\overline{\mathbf{Y}}=\overline{\mathbf{H}}\left(\begin{array}{l}
\mathcal{X} \\
\mathcal{F}
\end{array}\right)+\overline{\mathbf{E}}
$$

where $\overline{\mathbf{Y}}=\left(\mathbf{x}_{0}^{\top}, \mathbf{0}^{\top}, \mathcal{Y}^{\top}, \mathbf{0}^{\top}\right)^{\top}, \operatorname{Cov}(\overline{\mathbf{E}})=\operatorname{diag}\left(\mathbf{P}_{0}, \mathbf{Q}_{1}, \ldots, \mathbf{Q}_{K}, \mathbf{R}_{1}, \ldots, \mathbf{R}_{k}, \tilde{\mathbf{K}}_{f f}\right)$ and the sparse matrix $\overline{\mathbf{H}}$ follows naturally. Using the Markov property of the observation the joint probability distribution is given by

$$
\begin{aligned}
p(\mathcal{Y}, \mathcal{X}, \mathcal{F})= & p(\mathcal{Y} \mid \mathcal{X}) p(\mathcal{X} \mid \mathcal{F}) p(\mathcal{F}) \\
= & \mathcal{N}\left(\mathbf{x}_{0} \mid \overline{\mathbf{x}}_{0}, \mathbf{P}_{0}\right) \mathcal{N}\left(\mathcal{F} \mid \mathbf{0}, \tilde{\mathbf{K}}_{f f}\right) \prod_{k=1}^{K} \mathcal{N}\left(\mathbf{y}_{k} \mid \mathbf{C}_{k} \mathbf{x}_{k}, \mathbf{R}_{k}\right) \times \\
& \prod_{k=1}^{K} \mathcal{N}\left(\mathbf{x}_{k} \mid \mathbf{A}_{k} \mathbf{x}_{k-1}+\mathbf{B}_{k} \mathbf{f}_{k-1}, \mathbf{Q}_{k}\right) .
\end{aligned}
$$

The joint posterior distribution between the states and the unknown function is given by $p(\mathcal{X}, \mathcal{F} \mid \mathcal{Y}) \propto p(\mathcal{Y}, \mathcal{X}, \mathcal{F})$. Manipulating the product of Gaussians results in the smoothing posterior

$$
\left(\begin{array}{l}
\mathcal{X} \\
\mathcal{F}
\end{array}\right) \mid \mathcal{Y} \sim \mathcal{N}\left(\left(\overline{\mathbf{H}}^{\top} \overline{\mathbf{R}}^{-1} \overline{\mathbf{H}}\right)^{-1} \overline{\mathbf{H}}^{\top} \overline{\mathbf{R}}^{-1} \overline{\mathbf{Y}}, \overline{\mathbf{H}}^{\top} \overline{\mathbf{R}}^{-1} \overline{\mathbf{H}}\right)
$$

Similar calculations can be used to obtain the posterior distribution for the remaining linear models in Sections 3.9.2-3.9.4. However, their structures lend themselves to recursive estimation algorithms that are more efficient since they do not require the knowledge of all inputs in advance.

\subsubsection{Recursive Estimation Using Extended Kalman Filter}

The EKF [164] is an algorithm that recursively assumes a Gaussian approximation for the prediction and filter distributions [159], denoted by

$$
\left(\begin{array}{c}
\mathcal{X}_{k} \\
\mathcal{W}_{k}
\end{array}\right) \mid \mathcal{Y}_{1: k-1} \sim \mathcal{N}\left(\left(\begin{array}{c}
\overline{\mathcal{X}}_{k} \\
\overline{\mathcal{W}}_{k}
\end{array}\right),\left(\begin{array}{cc}
\overline{\mathbf{P}}_{x, k} & \overline{\mathbf{P}}_{w x, k} \\
\overline{\mathbf{P}}_{w x, k}^{\top} & \overline{\mathbf{P}}_{w, k}
\end{array}\right)\right)
$$




$$
\left(\begin{array}{c}
\mathcal{X}_{k} \\
\mathcal{W}_{k}
\end{array}\right) \mid \mathcal{Y}_{1: k} \sim \mathcal{N}\left(\left(\begin{array}{c}
\hat{\mathcal{X}}_{k} \\
\hat{\mathcal{W}}_{k}
\end{array}\right),\left(\begin{array}{cc}
\mathbf{P}_{x, k} & \mathbf{P}_{w x, k} \\
\mathbf{P}_{w x, k}^{\mathrm{T}} & \mathbf{P}_{w, k}
\end{array}\right)\right),
$$

where the notation $\overline{\mathcal{X}}_{k}=\left(\overline{\mathbf{x}}_{k}^{1 \top}, \ldots, \overline{\mathbf{x}}_{k}^{I \top}\right)^{\top}$ and $\hat{\mathcal{X}}_{k}=\left(\hat{\mathbf{x}}_{k}^{1 \top}, \ldots, \hat{\mathbf{x}}_{k}^{I \top}\right)^{\top}$ is used and $\mathcal{Y}_{1: k}$ denotes all measurements up to time step $k$. Each iteration consists of a time update step and a measurement update step, in which the transition and observation functions are linearized, respectively. For linear models, the EKF reduces to the $\mathrm{KF}$.

\section{Time Update}

The transition function for the dynamical states in (3.84b) is linear in the inducing states and the process noise, but nonlinear in the dynamical states. The Jacobian with regards to the dynamical states is given by

$$
\begin{array}{r}
\frac{\partial \mathbf{x}_{k}^{i}}{\partial \mathbf{x}_{k-1}^{i}}=\mathbf{A}_{k}+\mathbf{B}_{k}\left(\sum_{l=1}^{L} \mathbf{w}_{k-1}^{l} \mathbf{K}_{\mathbf{z}}\left(\mathbf{D}_{k} \mathbf{x}_{k-1}^{i}, \mathbf{z}_{l}^{u}\right)+\right. \\
\left.\mathbf{v}_{k}^{i f}\left(\mathbf{D}_{k} \mathbf{x}_{k-1}^{i}\right) \frac{\Lambda_{\mathbf{z}}\left(\mathbf{D}_{k} \mathbf{x}_{k-1}^{i}\right)}{2 \Lambda\left(\mathbf{D}_{k} \mathbf{x}_{k-1}^{i}\right)}\right) \mathbf{D}_{k},
\end{array}
$$

which is used to define

$$
\begin{aligned}
\mathbf{F}_{x}^{i} & \left.\triangleq \frac{\partial \mathbf{x}_{k}^{i}}{\partial \mathbf{x}_{k-1}^{i}}\right|_{\mathbf{x}_{k-1}^{i}=\hat{\mathbf{x}}_{k-1}^{i}, \mathcal{W}_{k-1}=\hat{\mathcal{W}}_{k-1}, \mathbf{v}_{k}^{i f}\left(\mathbf{D}_{k} \mathbf{x}_{k-1}^{i}\right)=\mathbf{0}} \\
& =\mathbf{A}_{k}+\mathbf{B}_{k}\left(\sum_{l=1}^{L} \hat{\mathbf{w}}_{k}^{l} \mathbf{K}_{\mathbf{z}}\left(\mathbf{D}_{k} \hat{\mathbf{x}}_{k-1}^{i}, \mathbf{z}_{l}^{u}\right)\right) \mathbf{D}_{k \prime} \\
\mathbf{F}_{w}^{i} & \left.\triangleq \frac{\partial \mathbf{x}_{k}^{i}}{\partial \mathcal{W}_{k-1}}\right|_{\mathbf{x}_{k-1}^{i}=\hat{\mathbf{x}}_{k-1}^{i}, \mathcal{W}_{k-1}=\hat{\mathcal{W}}_{k-1}}=\mathbf{B}_{k} \tilde{\mathbf{K}}_{\cdot u}\left(\mathbf{D}_{k} \mathbf{x}_{k-1}^{i}\right),
\end{aligned}
$$

where the time index $k$ is left out for brevity and Proposition 5.5 is used.

Proposition 5.5: Given the stochastic variable

$$
\mathbf{v} \sim \mathcal{N}(\boldsymbol{\mu}(\mathbf{z}), \lambda(\mathbf{z}) \mathbf{I}),
$$

the Jacobian with regards to $\mathbf{z}$ is given by

$$
\begin{aligned}
\frac{\partial \mathbf{v}}{\partial \mathbf{z}} & =\frac{\partial \mu}{\partial \mathbf{z}}+(\mathbf{v}-\boldsymbol{\mu}(\mathbf{z})) \frac{1}{2 \lambda(\mathbf{z})} \frac{\partial \lambda(\mathbf{z})}{\partial \mathbf{z}} \\
& =\frac{\partial \mu}{\partial \mathbf{z}}+\frac{1}{2}(\mathbf{v}-\boldsymbol{\mu}(\mathbf{z})) \frac{\partial}{\partial \mathbf{z}}(\log (\lambda(\mathbf{z}))
\end{aligned}
$$

Proof: Rewrite the stochastic variable as

$$
\mathbf{v}=\boldsymbol{\mu}(\mathbf{z})+\sqrt{\lambda(\mathbf{z})} \cdot \mathbf{n},
$$


where $\mathbf{n} \sim \mathcal{N}(\mathbf{0}, \mathbf{I})$, then

$$
\begin{aligned}
\frac{\partial \mathbf{v}}{\partial \mathbf{z}} & =\frac{\partial \mu}{\partial \mathbf{z}}+\mathbf{n} \frac{1}{2 \sqrt{\lambda(\mathbf{z})}} \frac{\partial \lambda(\mathbf{z})}{\partial \mathbf{z}} \\
& =\frac{\partial \mu}{\partial \mathbf{z}}+(\mathbf{v}-\boldsymbol{\mu}(\mathbf{z})) \frac{1}{2 \lambda(\mathbf{z})} \frac{\partial \lambda(\mathbf{z})}{\partial \mathbf{z}} \\
& =\frac{\partial \mu}{\partial \mathbf{z}}+\frac{1}{2}(\mathbf{v}-\boldsymbol{\mu}(\mathbf{z})) \frac{\partial}{\partial \mathbf{z}}(\log (\lambda(\mathbf{z}))
\end{aligned}
$$

The time update step in the EKF is then given by

$$
\begin{aligned}
\overline{\mathbf{x}}_{k}^{i}= & \mathbf{A}_{k} \hat{\mathbf{x}}_{k-1}^{i}+\mathbf{B}_{k} \tilde{\mathbf{K}}_{\cdot u}\left(\mathbf{D}_{k} \hat{\mathbf{x}}_{k-1}^{i}\right) \hat{\mathcal{W}}_{k-1}, \\
\overline{\mathcal{W}}_{k}= & \mathbf{G}_{k} \hat{\mathcal{W}}_{k-1}+\boldsymbol{\mu}_{k^{\prime}} \\
\overline{\mathbf{P}}_{x, k}^{i, n}= & \mathbf{F}_{x}^{i} \mathbf{P}_{x, k-1}^{i, n}\left(\mathbf{F}_{x}^{n}\right)^{\top}+\mathbf{F}_{w}^{i} \mathbf{P}_{w x, k-1}^{\cdot, n}\left(\mathbf{F}_{x}^{n}\right)^{\top}+\mathbf{F}_{x}^{i}\left(\mathbf{F}_{w}^{n} \mathbf{P}_{w x, k-1}^{, i}\right)^{\top}+ \\
& \mathbf{F}_{w}^{i} \mathbf{P}_{w, k-1}\left(\mathbf{F}_{w}^{n}\right)^{\top}++\left(\mathbf{B}_{k} \Lambda\left(\mathbf{D}_{k} \hat{\mathbf{x}}_{k}^{i}\right)\left(\mathbf{B}_{k}\right)^{\top}+\mathbf{Q}_{k}^{i}\right) \delta_{i n}, \\
\overline{\mathbf{P}}_{w x, i}^{j, i}= & \mathbf{G}^{j,} \mathbf{P}_{w x, k-1}^{\cdot, i}\left(\mathbf{F}_{x}^{i}\right)^{\top}+\mathbf{G}^{j,} \mathbf{P}_{w, k-1}\left(\mathbf{F}_{w}^{i}\right)^{\top}, \\
\overline{\mathbf{P}}_{w, k}^{j, l}= & \mathbf{G}^{j,} \mathbf{P}_{w, k-1}\left(\mathbf{G}^{l, \cdot}\right)^{\top}+\boldsymbol{\Sigma}_{k}^{j, l},
\end{aligned}
$$

where $i \in\{1: I\}$ and $n=\in\{1: I\}$ index submatrices corresponding to dynamic states, $j=\in\{1: L\}$ and $l=\in\{1: L\}$ index submatrices corresponding to inducing states and $\delta_{i n}$ is the Kronecker delta. The system function for the inducing states in (3.84e) is linear in all states and the noise.

\section{Measurement Update}

The measurement update step in the EKF is given for a single observation by

$$
\begin{aligned}
& \mathbf{S}_{k}^{i} \triangleq \mathbf{C}_{k} \overline{\mathbf{P}}_{x, k}^{i, i} \mathbf{C}_{k}^{\top}+\mathbf{R}_{k}^{i}, \\
& \mathbf{G}_{x, k}^{i} \triangleq \overline{\mathbf{P}}_{x, k}^{, i} \mathbf{C}_{k}^{\top}\left(\mathbf{S}_{k}^{i}\right)^{-1}, \\
& \mathbf{G}_{w, k}^{i} \triangleq \overline{\mathbf{P}}_{w x, k}^{, i} \mathbf{C}_{k}^{\top}\left(\mathbf{S}_{k}^{i}\right)^{-1}, \\
& \hat{\mathcal{X}}_{k}=\overline{\mathcal{X}}_{k}+\mathbf{G}_{x, k}^{i}\left(\mathbf{y}_{k}^{i}-\mathbf{C}_{k} \overline{\mathbf{x}}_{k}^{i}\right), \\
& \hat{\mathcal{W}}_{k}=\overline{\mathcal{W}}_{k}+\mathbf{G}_{w, k}^{i}\left(\mathbf{y}_{k}^{i}-\mathbf{C}_{k} \overline{\mathbf{x}}_{k}^{i}\right), \\
& \mathbf{P}_{x, k}=\overline{\mathbf{P}}_{x, k}-\mathbf{G}_{x, k}^{i} \mathbf{S}_{k}^{i}\left(\mathbf{G}_{x, k}^{i}\right)^{\top}, \\
& \mathbf{P}_{w x, k}=\overline{\mathbf{P}}_{w x, k}-\mathbf{G}_{w, k}^{i} \mathbf{S}_{k}^{i}\left(\mathbf{G}_{x, k}^{i}\right)^{\top}, \\
& \mathbf{P}_{w, k}=\overline{\mathbf{P}}_{w, k}-\mathbf{G}_{w, k}^{i} \mathbf{S}_{k}^{i}\left(\mathbf{G}_{w, k}^{i}\right)^{\top} .
\end{aligned}
$$

The measurement update is applied for each observation in turn, using the filter distribution from the previous measurement update as the prediction distribution. 
Table 5.3: The RMSE results of the simulations.

\begin{tabular}{cccccccc}
\hline \multicolumn{2}{c}{ Configuration } & \multicolumn{3}{c}{ Position RMSE $(\mathrm{m})$} & \multicolumn{3}{c}{ Velocity RMSE $(\mathrm{m} / \mathrm{s})$} \\
$\mathbf{S}$ & $\mathbf{q}(\mathrm{m} / \sqrt{\mathrm{s}})$ & NCVM & NCAM & GPMM & NCVM & NCAM & GPMM \\
\hline 1 & 2 & 2.66 & 2.53 & 2.13 & 2.94 & 2.15 & 0.69 \\
1 & 20 & 2.99 & 3.01 & 2.97 & 26.26 & 36.78 & 2.36 \\
1 & 50 & 3.02 & 3.13 & 2.99 & 64.27 & 89.73 & 4.88 \\
2 & 2 & 4.24 & 3.99 & 2.86 & 2.83 & 2.55 & 0.84 \\
5 & 0 & 6.67 & 8.49 & 3.77 & 2.42 & 3.29 & 1.23 \\
5 & 0.5 & 6.74 & 8.59 & 3.87 & 2.43 & 3.32 & 1.23 \\
5 & 2 & 7.77 & 10.01 & 4.91 & 2.68 & 3.85 & 1.22 \\
5 & 5 & 11.98 & 15.74 & 8.29 & 3.79 & 6.00 & 1.42 \\
5 & 20 & 40.41 & 71.93 & 29.05 & 11.96 & 29.52 & 3.19 \\
5 & 50 & 101.46 & 177.33 & 71.29 & 30.03 & 72.66 & 5.83 \\
10 & 2 & 18.9 & 26.88 & 10.77 & 3.92 & 5.84 & 1.86 \\
\hline
\end{tabular}

Application 5.10: Velocity Field Estimation

The method presented in this section is applied to the data simulated as described in Application 3.10. The results for a realization of one configuration is shown in Figure 5.16.

The RMSE of the position and velocity for each estimator and each configuration is presented in Table 5.3. It is clear that the proposed method performs better than the conventional models in this scenario. However, the performance gain reduces with increasing observation frequency and increasing process noise. The average normalized estimation error squared (ANEEs), see Table 5.4, also shows that the proposed estimator is in general consistent, unlike the estimators using the conventional models. Two notable exceptions in Table 5.4 are the configurations with no process noise and a long time between the observations. In the first case, the noise introduced by the GP will have a larger relative effect on the estimate, causing inconsistencies. In the second case the use of a noisy input to the GP starts having effect. The velocity computed for the proposed method is obtained as the predicted velocity at the estimated location of the target. The GPMM is successfully used to estimate the underlying velocity field as well as improve the predictions made by the model for tracking. This concludes the velocity field scenario.

\subsubsection{Implementation Considerations}

Direct implementation of the equations in the previous steps may be expensive in the general case, even for a moderate grid size and a moderate number of dynamical systems since the cross-covariances between all states are maintained. However, the potential improvement over models disregarding the influence lies in the cross-covariances. Methods are therefore proposed in this section to only 


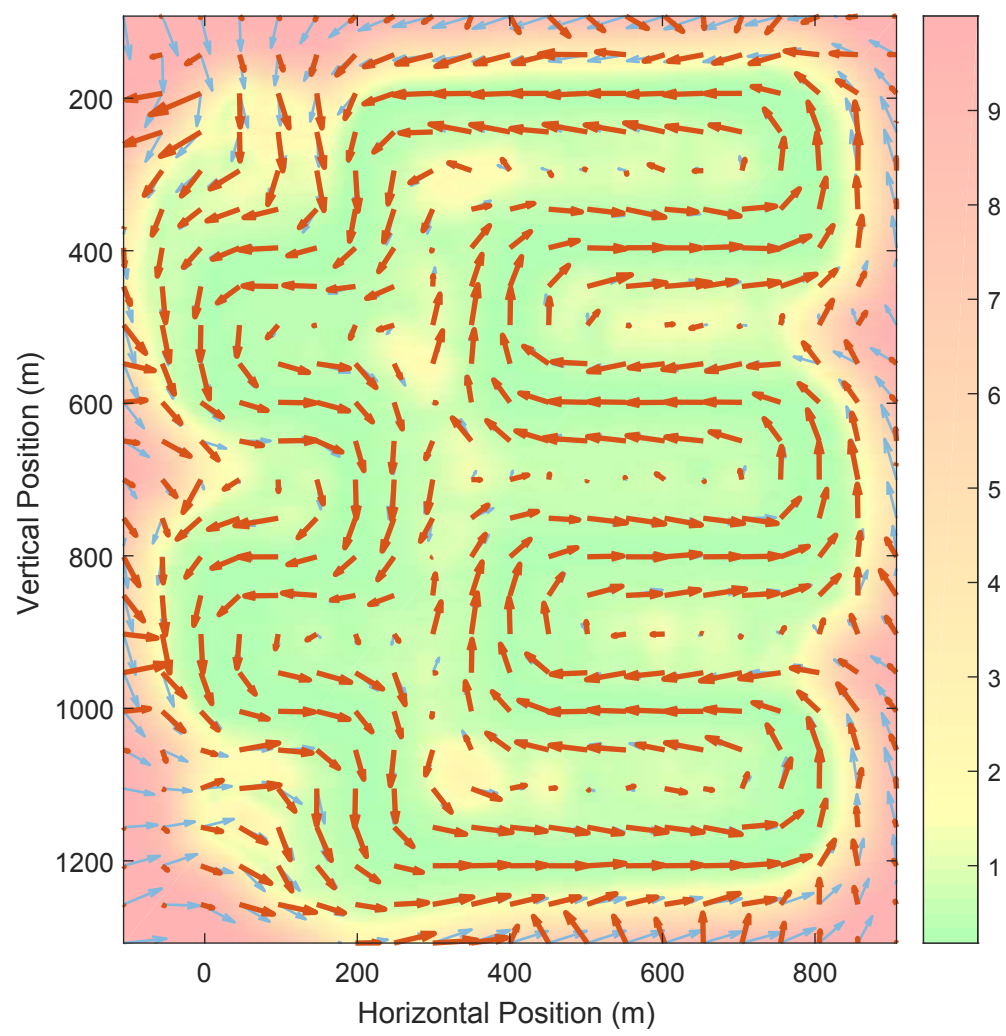

Figure 5.16: The true and estimated velocity field in blue and red, respectively, for the configuration $q=0.5 \mathrm{~m} / \sqrt{\mathrm{s}}$ and $S=5$, where the length of the arrows corresponds to the distance travelled in $5 \mathrm{~s}$. The estimated velocity appears to converge to the true velocity along the path, but approaches the prior of zero velocity where fewer observations are available. The standard deviation of the velocity $(\mathrm{m} / \mathrm{s})$ is shown in the background. 
Table 5.4: The ANEES results of the simulations.

\begin{tabular}{ccccc}
\hline \multicolumn{2}{c}{ Configuration } & \multicolumn{3}{c}{ ANEES } \\
$\mathbf{S}$ & $\mathbf{q}(\mathrm{m} / \sqrt{\mathrm{s}})$ & NCVM & NCAM & GPMM \\
\hline 1 & 2 & 0.93 & 1.05 & 1.00 \\
1 & 20 & 1.00 & 1.01 & 1.00 \\
1 & 50 & 1.02 & 1.09 & 1.00 \\
2 & 2 & 0.78 & 1.05 & 1.00 \\
5 & 0 & 0.33 & 0.67 & 1.16 \\
5 & 0.5 & 0.33 & 0.69 & 1.06 \\
5 & 2 & 0.37 & 0.89 & 1.03 \\
5 & 5 & 0.6 & 2.02 & 1.01 \\
5 & 20 & 4.72 & 1.51 & 1.01 \\
5 & 50 & 0.56 & 8.13 & 1.00 \\
10 & 2 & 0.22 & 0.43 & 1.30 \\
\hline
\end{tabular}

maintain and update significant cross-covariances.

\section{Covariance Function Truncation}

By expanding the matrix multiplications in (5.80c) it is noted that all terms that introduce cross-covariances in the time update step directly scale with the kernel value. The contribution to the cross-covariances by terms where the kernel value is small is negligible. By truncating the normalized kernel,

$$
\tilde{K}\left(\mathbf{x}, \mathbf{x}^{\prime}\right)= \begin{cases}K\left(\mathbf{x}, \mathbf{x}^{\prime}\right), & \sigma^{2} K\left(\mathbf{x}, \mathbf{x}^{\prime}\right)<\epsilon_{K}, \\ 0, & \text { otherwise, }\end{cases}
$$

the number of terms in the computation decreases and small cross-covariances reduce to exactly zero. The risk is that the covariance matrix loses positivedefiniteness [169], so the threshold $\epsilon_{K}$ must be set low enough. This improves the performance with negligible effect on the results if the length parameter is relatively small and $\epsilon_{K}$ is appropriately chosen.

\section{Inducing Input Grid}

The number of inducing variables, and thus the required memory and computations, directly depends on the resolution of the inducing input grid. The resolution is a trade-off between the sparsity of the function and the performance. If the resolution is too fine, the covariance between the inducing variables is large and less information is stored per variable. If it is too coarse, the inducing variables struggle to represent the underlying function.

Since the inputs scale with the length parameter $\theta$ in (3.85) the distance between the inducing inputs is selected relative to this parameter. Simple tests were performed for one dimensional inputs comparing the Frobenius norm of 
the covariance matrices $\mathbf{K}_{f f}$ and $\mathbf{Q}_{f f}$, as $\left\|\mathbf{K}_{f f}-\mathbf{Q}_{f f}\right\|_{F} /\left\|\mathbf{K}_{f f}\right\|_{F}$, for $\mathbf{z}_{l}^{u}$ gridded relative to the length parameter and random $\mathbf{z}_{k}^{f}$. They indicate that the error is insignificant for a grid distance less than $\theta / 3$, around $1 \%$ for a distance around $\theta$ and growing fast for distances greater than $\theta$. As a trade-off between performance and computational burden, the grid distance is proposed to be chosen in the interval $[\theta / 3, \theta]$.

Remark 5.1: One might contemplate allowing inducing points to be added, removed or moved, as suggested in [29]. This is unfortunately not as straightforward as it might seem. Let $\mathbf{z}_{\hat{u}}$ denote added inducing inputs and $\mathbf{z}_{\bar{u}}$ the augmented set of original and added inducing inputs. The weights for the new set of inducing variables are given by

$$
\overline{\mathcal{W}}=\tilde{\mathbf{K}}_{\bar{u} \bar{u}}^{-1} \tilde{\mathbf{K}}_{\bar{u} u} \mathcal{W}=\left(\begin{array}{ll}
\mathbf{K}_{u u} & \mathbf{K}_{u \hat{u}} \\
\mathbf{K}_{\hat{u} u} & \mathbf{K}_{\hat{u} \hat{u}}
\end{array}\right)^{-1}\left(\begin{array}{l}
\mathbf{K}_{u u} \\
\mathbf{K}_{\hat{u} u}
\end{array}\right) \mathcal{W}=\left(\begin{array}{l}
\mathbf{I} \\
\mathbf{0}
\end{array}\right) \mathcal{W} .
$$

The new inducing variables are identically zero, i.e., degenerate Gaussian with zero mean and zero variance, and will therefore not improve the flexibility of the function estimate. Adding new observations will not improve the situation. Removing inducing variables reduces the flexibility of the function estimate. Consequently, modifying the set of inducing variables by simply adding and removing variables will eventually result in a function estimate that approaches identically zero. The set of inducing variables is therefore assumed to be fixed.

\section{Cross-Covariance Thresholding}

Relevant cross-covariances might decrease over time, so a mechanism to threshold and effectively remove small cross-covariances would reduce the computational cost. To obtain a method that is independent of the state variances it is proposed that thresholding is performed in the correlation matrix,

$$
\mathbf{C}=\mathbf{D}^{-1} \mathbf{P D}^{-1}
$$

where $\mathbf{D}^{2}=\operatorname{diag}(\mathbf{P})$, to determine which cross-covariances to remove. However, removing cross-covariances does not guarantee that the resulting covariance matrix remains positive definite. By setting the threshold very low the risk is mitigated in practice, while still allowing almost zero cross-covariances to be disregarded in computations. To handle a larger threshold while still guaranteeing positive definiteness, the covariance matrix needs to be compensated for the removed cross-covariances.

A method is proposed based on the idea that the sum of a positive-definite matrix and a positive-semi-definite matrix is positive definite. For the removal of a single cross-covariance $\mathbf{P}_{i j}=\mathbf{P}_{j i}$, the sparse approximation of the covariance matrix is computed as

$$
\tilde{\mathbf{P}}=\mathbf{P}+\tilde{\mathbf{I}}_{i j}^{\top} \mathbf{E} \tilde{\mathbf{I}}_{i j},
$$

where the selector matrix is defined as $\mathbf{I}_{i j}\left(x_{1}, \ldots, x_{n}\right)^{\top}=\left(x_{i}, x_{j}\right)^{\top}$ and

$$
\mathbf{E}=\left(\begin{array}{cc}
\sqrt{\frac{\mathbf{P}_{i i}}{\mathbf{P}_{j j}}}\left|\mathbf{P}_{i j}\right| & -\mathbf{P}_{i j} \\
-\mathbf{P}_{i j} & \sqrt{\frac{\mathbf{P}_{j j}}{\mathbf{P}_{i i}}}\left|\mathbf{P}_{i j}\right|
\end{array}\right)
$$


The two diagonal elements of $\mathbf{E}$ are chosen to achieve a rank one positive semidefinite matrix such that the resulting determinant of the covariance matrix $\tilde{\mathbf{I}}_{i j} \tilde{\mathbf{P}} \tilde{\mathbf{I}}_{i j}^{\top}$ is minimized.

\section{Gating Inputs to Determine Significant Covariances}

To further reduce the computational burden, it would be preferable to avoid computing cross-covariances, which are the major bottle-neck in the filter, in the first place.

The first difficulty is to determine which cross-covariances will be small. A simple option to determine which cross-covariances are potentially significant is to require a maximum distance, similar to a gate, weighted by the length parameter, between the function inputs of the processes.

The second difficulty is how to compensate the diagonal of the covariance matrix for the removal of a cross-covariance when its magnitude is unknown, to avoid losing positive definiteness. A simple option is to not compensate and make sure the minimum distance is large enough and slightly increase the process covariance. A more complicated option is to estimate the compensation by computing a subset of the cross-covariances or computing them at a lower frequency.

\section{Symmetry, Sparsity and Reuse Considerations}

The covariance matrix is symmetric, hence storing and updating the entire $\mathbf{P}_{x, k}$ and $\mathbf{P}_{w, k}$ matrices result in double storage and double computation. An efficient method to insert, store, access and remove individual covariances and crosscovariances would reduce the required memory and computation. Especially if the majority of the cross-covariances are close to zero. Many numerical libraries provide efficient implementations for symmetric and sparse matrices. Often it is worthwhile to intelligently select the order of computations and quantities to reuse to avoid redundancy.

\section{Application 5.11: Sea Ice Learning Estimation}

Detections in the sea ice dataset are obtained as described in Application 4.4. The purpose of the example is to evaluate the motion model, hence data association is not considered in the evaluation. A global nearest neighbour (GNN) tracker [31] is applied on the full dataset using a NCVM [114]. Detections associated to tracks of at least $30 \mathrm{~min}$ are stored. For each track $30 \%$ of the detections are kept to simulate a lower sampling rate.

The hyperparameters are optimized over $q \in\{42.4, \underline{141.4}, 424.3\} \mathrm{m} / \sqrt{\mathrm{h}}^{3}$ for the NCVM, over $q \in\{84.9, \underline{282.8}, 848.5\} \mathrm{m} / \sqrt{\mathrm{h}}^{5}$ for the NCAM and for both models over $r \in\{5, \underline{15}, 50\} \mathrm{m}$ with regards to maximum likelihood, with the optimal parameters underlined. For the proposed model the hyperparameters are optimized over $q \in\{42.4, \underline{141.4}, 424.3\}, \theta=\delta \in\{500, \underline{750}, 1000\} \mathrm{m}$, $\sigma \in\{63.2, \underline{200}, 632.5\} \mathrm{m} / \mathrm{h}^{2}$ and $\alpha \in\{0.01, \underline{0.05}\}$, with the optimal parameters underlined. Since the true ice object locations are not available, the prediction 
error method (PEM) [115] is used to compare the models. The method is applied both with a quadratic norm for the prediction errors and in the maximum likelihood form. The results are presented in Table 5.5, showing the negative $\log$-likelihoods for the latter. The proposed method performs slightly better in this setup compared to the conventional models. The tracks and the estimated acceleration function for a single instant of time is presented in Figure 5.17.

Using the various methods for implementation considered in this section, the computation time reduces significantly and it is feasible to run an optimization algorithm. The particular approximations used are zeroing of cross-correlation blocks with an absolute sum less than $1 \cdot 10^{-4}$ and gating of targets and inducing inputs within a distance of $2.1 \theta$ and $5.7 \theta$, respectively. The log-likelihood is optimized over the parameters $q$ and $r$ for the NCVM, resulting in $q=161.3$ and $r=12.4 \mathrm{~m}$. The same parameters are used for the GPMM, which is then optimized over $\theta, \delta=2 \theta / 3$ and $\sigma$, resulting in $\theta=739 \mathrm{~m}$ and $\sigma=3.5 \mathrm{~m} / \mathrm{h}^{2}$. The negative log-likelihood is 382359 and 380522 for the NCVM and GPMM, respectively, showing slightly better performance for the proposed method. The practical use of the GPMM on real data has been demonstrated. This concludes the sea ice learning application.

Table 5.5: Prediction errors for the sea ice learning application.

\begin{tabular}{lccc}
\hline Method & NCVM & NCAM & GPMM \\
\hline Quadratic Norm & 6.13 & 7.27 & 6.00 \\
Maximum Likelihood & 385877 & 403723 & 383336 \\
\hline
\end{tabular}




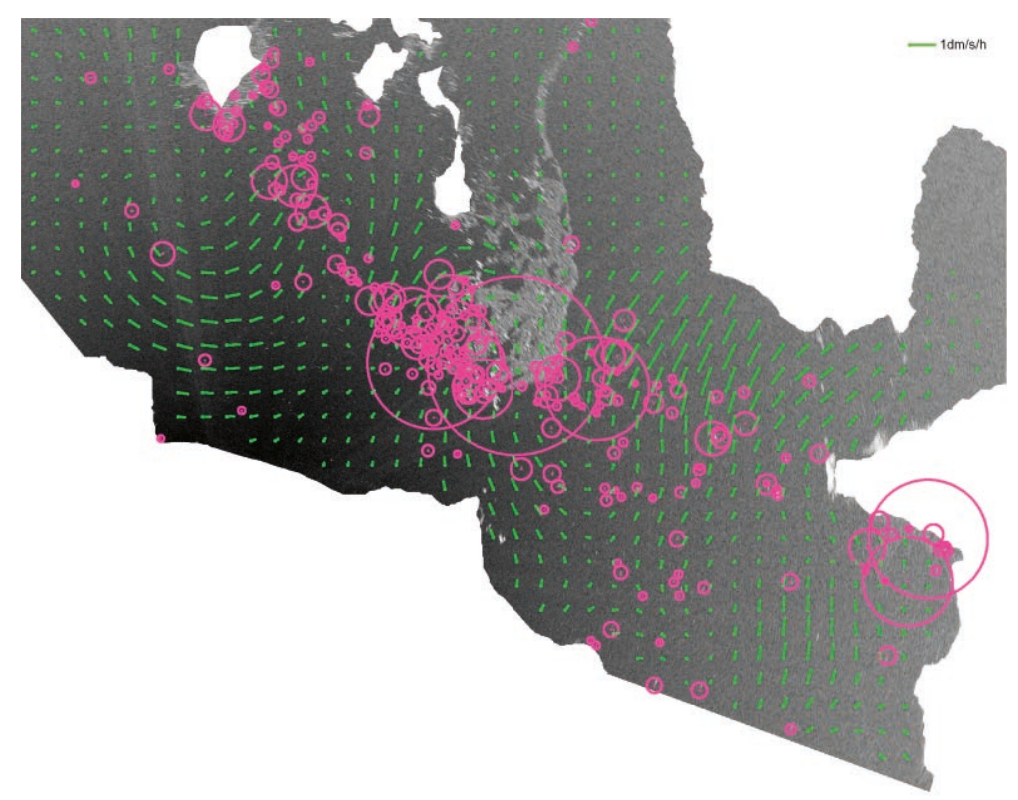

Figure 5.17: The tracks and current acceleration estimation of the sea ice learning application. 


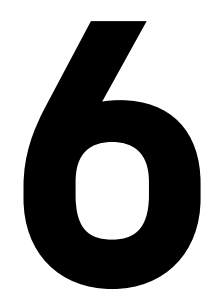

\section{Data Association}

Data association in the context of target tracking is the problem of associating a sequence of measurements to form tracks when there is an uncertainty in association. Association uncertainty arises when multiple targets are present in the scene, targets appear and disappear, or when false detections occur. The brute-force approach is to consider all possible associations over time between measurements and select the most probable hypothesis given the target models. The combinatorial complexity of this approach is intractable, so several established methods have been developed that strive to reduce this complexity without degrading performance more than necessary. They vary in complexity, performance and options for tuning, allowing the designer to select a suitable approach for meeting the requirements of a particular application. There is an abundance of literature covering the topic of data association and target tracking, especially with a focus on military applications. Common principles and an overview of established methods are described in, e.g., [11, 31]. For more details on implementation in particular applications, see e.g. $[9,12,17]$. More modern methods are described in, e.g., [121, 168].

An outline of data association uncertainty and how it relates to target tracking is given in Section 1.1. Concepts related to data association uncertainty are described in Section 6.1. The target tracking algorithms multiple hypothesis tracker (MHT), probabilistic data association (PDA) filter and labelled multi-Bernoulli (LMB) filter, for solving the data association problem are presented in Sections 6.2, 6.3 and 6.4, respectively. A modification of the PDA filter, first described by the author in [181], is presented in Section 6.3.3. A reformulation of the LMB filter is proposed in Section 6.4.3. 


\subsection{Data Association Uncertainty}

Given a set of existing tracks and an unordered set of detections, commonly denoted a scan, at each time step, the task is to associate the measurements to existing tracks, new tracks or identify them as false detections. A track is represented by its posterior distribution, given a target model, to allow for efficient association, state estimation and quality evaluation. The relevant elements are modelled probabilistically to allow for a comparison between different association hypotheses. These concepts and a formulation of the assignment problem are briefly described in this section. More details on the subject are given in, e.g., [31].

\subsubsection{Uncertainty Models}

In this section, a few common models for the uncertainty of false detections, new tracks and missed detections are briefly described. Standard models are outlined for these concepts that, with slight variations, will be used subsequently in the chapter. For a more detailed description, see e.g. [31].

At the current time step each target in the field of view generates a single detection with probability $P_{D}$. This results in a set $\mathcal{Y}_{e}=\left\{\mathbf{y}_{i}^{e}\right\}_{i=1}^{M_{e}}$ of measurements for existing targets. The number $M_{f}$ of false detections is modelled with a Poisson distribution as

$$
p\left(M_{f}\right)=\mathcal{P} \mathcal{S}\left(M_{f} \mid V \beta_{f}\right)=\frac{\left(V \beta_{f}\right)^{M_{f}} e^{-V \beta_{f}}}{M_{f} !},
$$

where $\beta_{f}$ is the false detection density and $V$ is the size of the surveillance volume $\mathcal{V}$. This results in a set $\mathcal{Y}_{f}=\left\{\mathbf{y}_{i}^{f}\right\}_{i=1}^{M_{f}}$ of false detections. Each false detection is independently and uniformly distributed over the surveillance volume $\mathcal{V}$ as

$$
p\left(\mathbf{y}_{i}^{f}\right)=\mathcal{U}\left(\mathbf{y}_{i}^{f} \mid \mathcal{V}\right)= \begin{cases}\frac{1}{V}, & \text { if } \mathbf{y}_{i}^{f} \in \mathcal{V}, \\ 0 . & \text { if } \mathbf{y}_{i}^{f} \notin \mathcal{V},\end{cases}
$$

New targets are similarly modelled as $\mathcal{Y}_{n}=\left\{\mathbf{y}_{i}^{n}\right\}_{i=1}^{M_{n}}$ with the new target density $\beta_{n}$ over the surveillance volume $\mathcal{V}$. The union of all measurements is reordered to discard the origins and delivered at time step $k$ as $\mathcal{Y}_{k}=\mathcal{Y}_{e} \cup \mathcal{Y}_{f} \cup \mathcal{Y}_{n}=$ $\left\{\mathbf{y}_{k}^{i}\right\}_{i=1}^{M_{k}}$.

To handle the uncertainty in the origin of the measurements in the set $\mathcal{Y}_{k}$, hypotheses regarding the origins of the measurements are constructed. A hypothesis $\theta_{1: k}$ is a set of associations for all measurements $\mathcal{Y}_{1: k}=\left\{\mathcal{Y}_{i}\right\}_{i=1}^{k}$. In a hypothesis, each measurement is either associated to a track or identified as a false detection. Existing tracks are updated with the associated measurements and new tracks are initialized for each false detection. A set of tracks $\mathcal{T}_{k}$ is estimated given the measurements $\mathcal{Y}_{1: k}$, a hypothesis $\theta_{1: k}$ and target models.

A track $\tau \in \mathcal{T}_{k}$ is estimated using the measurements $\mathcal{Y}_{1: k}^{\tau}$ associated to the track according to a hypothesis. The posterior distribution for the current state 
of the track is given by $p\left(\mathbf{x}_{k} \mid \tau\right)=p\left(\mathbf{x}_{k} \mid \mathcal{Y}_{1: k}^{\tau}\right)$. For the LGSSM the predicted measurement for a track $\tau \in \mathcal{T}_{k-1}$ is given by

$$
p\left(\mathbf{y}_{k} \mid \tau\right)=\mathcal{N}\left(\mathbf{y}_{k} \mid \hat{\mathbf{y}}_{k}^{\tau}, \mathbf{S}_{k}^{\tau}\right),
$$

where $\hat{\mathbf{y}}_{k}^{\tau}$ and $\mathbf{S}_{k}^{\tau}$ are computed using the $\mathrm{KF}$ in Algorithm 5.1 for the measurements used to form track $\tau$. An approximation of the prediction distribution for an NSSM can be obtained using the EKF or the PF.

To reduce the complexity or as a necessary part of the method, the detections are often gated [31]. Gating essentially means that only detections that could reasonably be generated by a track are considered for association. Often a hierarchy of gates are used, with simple coarse gates at higher levels and a finer elliptical gate at the lowest level. Elliptical gates reject detections from consideration if the squared Mahalanobis distance to the predicted measurement,

$$
d_{\tau}^{2}\left(\mathbf{y}_{k}\right)=\left(\mathbf{y}_{k}-\hat{\mathbf{y}}_{k}^{\tau}\right)^{\top}\left(\mathbf{S}_{k}^{\tau}\right)^{-1}\left(\mathbf{y}_{k}-\hat{\mathbf{y}}_{k}^{\tau}\right) \sim \chi^{2}(m), \quad \tau \in \mathcal{T}_{k-1},
$$

is greater than a threshold $\gamma$, where $m$ is the dimension of $\mathbf{y}_{k}$. A fixed probability $P_{G}$ for the distribution can be used to select $\gamma$ or the threshold can be derived from the false detection and new track models [31].

\subsubsection{Track Score}

As measurements are associated to a track, a score is maintained for the track over time [31]. The track score function $L_{k}^{\tau}$ for a track $\tau \in \mathcal{T}_{k}$ is the logarithm of the likelihood ratio between the hypotheses $\theta_{T}^{\tau}$ and $\theta_{F}^{\tau}$ of the track $\tau$ being a true target and a false target, respectively, [31]

$$
L_{k}^{\tau}=\log \left(\frac{p\left(\theta_{T}^{\tau} \mid \tau\right)}{p\left(\theta_{F}^{\tau} \mid \tau\right)}\right) .
$$

The track score can be computed recursively, as derived in [18], where a new track is initialized as

$$
L_{1}^{\tau}=\log \left(\beta_{n}\right)-\log \left(\beta_{f}\right) .
$$

Let $D_{k}^{\tau}$ denote that a measurement, $\mathbf{y}_{k}^{\tau}$, is associated to a track $\tau \in \mathcal{T}_{k-1}$ at time step $k$ in an association hypothesis $\theta_{1: k}$. The track score for a track $\tau \in \mathcal{T}_{k-1}$ is then updated as

$$
L_{k}^{\tau}=L_{k-1}^{\tau}+ \begin{cases}\log \left(p\left(\mathbf{y}_{k}^{\tau} \mid \tau, \theta_{T}^{\tau}\right)\right)+\log \left(P_{D}\right)-\log \left(\beta_{f}\right), & \text { if } D_{k}^{\tau}, \\ \log \left(1-P_{D} P_{G}\right), & \text { if } \neg D_{k^{\prime}}^{\tau}\end{cases}
$$

where

$$
\begin{aligned}
\log \left(p\left(\mathbf{y}_{k}^{\tau} \mid \tau, \theta_{T}^{\tau}\right)\right) & =\log \mathcal{N}\left(\mathbf{y}_{k}^{\tau} \mid \hat{\mathbf{y}}_{k}^{\tau}, \mathbf{S}_{k}^{\tau}\right) \\
& =-\frac{1}{2} \log \left(\left|2 \pi \mathbf{S}_{k}^{\tau}\right|\right)-\frac{1}{2}\left(\mathbf{y}_{k}^{\tau}-\hat{\mathbf{y}}_{k}^{\tau}\right)^{\top}\left(\mathbf{S}_{k}^{\tau}\right)^{-1}\left(\mathbf{y}_{k}^{\tau}-\hat{\mathbf{y}}_{k}^{\tau}\right),
\end{aligned}
$$


for an LGSSM.

The probability of an association hypothesis is computed using the recursion [18]

$$
\begin{aligned}
& p\left(\theta_{1: k} \mid \mathcal{Y}_{1: k}\right) \propto p\left(\theta^{1: k-1} \mid \mathcal{Y}^{k-1}\right) . \\
&\left(\frac{\beta_{n}}{\beta_{f}}\right)^{M_{k}^{n}} \prod_{\tau \in \mathcal{T}_{k}^{D}} \frac{p\left(\mathbf{y}_{k}^{\tau} \mid \tau\right) P_{D}}{\beta_{f}} \cdot\left(1-P_{D} P_{G}\right)^{\mid \mathcal{T}_{k}} \cdot(
\end{aligned}
$$

where $\mathcal{T}_{k}^{D}=\left\{\tau \in \mathcal{T}_{k-1} \mid D_{k}^{\tau}\right\}$ is the set of all detected tracks, $M_{k}^{n}$ is the number of measurements associated with new tracks, $\left|\mathcal{T}_{k} \neg D\right|$ is the number of non-detected tracks and $p\left(\mathbf{y}_{k}^{\tau} \mid \tau\right)$ is the prediction distribution of track $\tau$ given in (6.3), according to the hypothesis $\theta_{1: k}$. Equivalently, the probability can be obtained from the track scores as

$$
p\left(\theta_{1: k} \mid \mathcal{Y}_{1: k}\right) \propto \exp \left(\sum_{\tau \in \mathcal{T}_{k}} L_{k}^{\tau}\right)
$$

\subsubsection{Track Quality}

Given a track it is important to determine its quality. Before a sufficient number of measurements have been associated to a track it is uncertain whether the track represents a true or false target. A sequential probability ratio test (SPRT) [193] can be performed to determine the status of the track and if sufficient evidence has been received. Essentially, an upper threshold $\gamma_{h}$ and a lower threshold $\gamma_{l}$ are applied to the track score. Exceeding the upper threshold results in confirmation of the track as a true target and falling below the lower threshold indicates a false target, resulting in the deletion of the track. The limits are computed as [31]

$$
\gamma_{h}=L_{1}+\log \left(\frac{1-P_{F D}}{P_{F C}}\right) \text { and } \gamma_{l}=L_{1}+\log \left(\frac{P_{F D}}{1-P_{F C}}\right),
$$

where $P_{F D}$ is the accepted probability of falsely deleting a track for a true target and $P_{F C}$ is the accepted probability of confirming a track for a false target. The thresholds are set relative to the initial track score. Initially, before sufficient evidence is available to confirm a track, the track is commonly denoted an initiator or a tentative track.

At a later stage, a target might disappear from the field of view, resulting in a sequence of missed detections for the track and eventually the track needs to be declared dead. Since a high quality track results in a high track score over time, it is slightly more difficult to set up a threshold for detecting a dead confirmed track. A common approach is to apply a threshold $\gamma_{d}$ relative to the maximum track score as

$$
\gamma_{d}=L_{k_{m}}+\log \left(P_{F D C}\right),
$$

where $k_{m}=\arg \max _{k} L_{k}$ and $P_{F D C}$ is the probability of falsely deleting an alive confirmed track. 


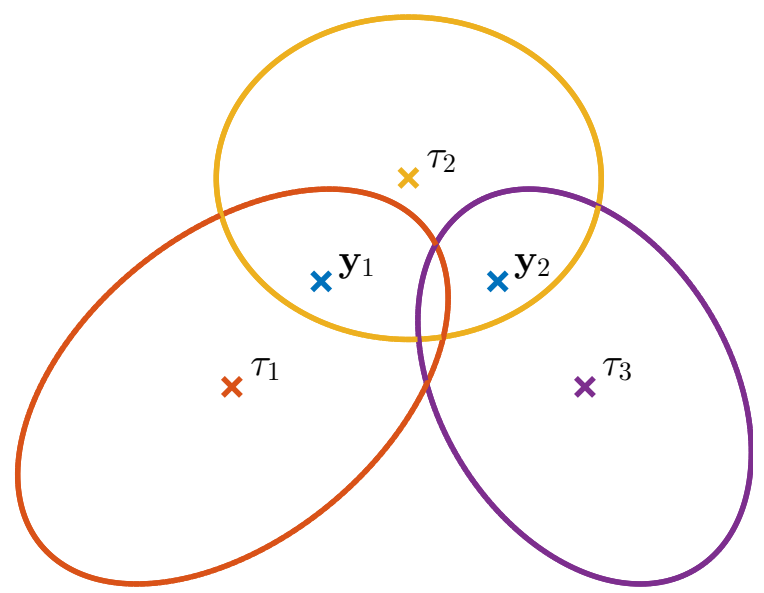

Figure 6.1: A simple scenario of three tracks with gates and two measurements.

An alternative to using track scores is to instead count the number of detections and missed detections of a track and set up heuristic rules for confirmation and deletion, commonly denoted $M / N$ logic [30]. For example, a track might be confirmed if it is detected $N_{1}$ times in a row and subsequently $N_{2}$ out of $M$ times, otherwise deleted. Similarly, a confirmed track is deleted after $N_{D}$ missed detections.

The two methods perform similarly and approximate conversions between thresholds and rules can be obtained. However, due to the model-based nature of SPRT, this approach is often preferable compared to using ad-hoc $M / N$ logic [31].

\subsubsection{Linear Assignment Problem}

Finding likely assignments of new measurements to existing tracks, new tracks and false detections is commonly called the linear assignment problem. At each time step a set $\mathcal{T}$ of $N$ tracks for each hypothesis, where each track is the result of earlier measurements and the hypothesis, is available and a set $\mathcal{Y}=\left\{\mathbf{y}_{i}\right\}_{i=1}^{M}$ of new measurements arrives. See Figure 6.1 for a simple scenario involving three tracks and two measurements that will be used to illustrate the concepts in this section. The goal is to assign each measurement to either one of the existing tracks, a new track or identify it as a false detection. The measurements are first gated for all tracks, conceptually resulting in a gating matrix, and then association probabilities are computed for all possible gated associations, resulting in an association matrix.

The gating matrix $\mathbf{G}$ is a logical matrix with one measurement per column and one track per row. If a measurement is inside the gate of a track, the corresponding element is set to true, otherwise it is set to false. An example of a gating matrix is shown in Table 6.1. An equivalent representation is the set $\mathcal{G}_{\tau}$ containing the measurement indices $i \in\{1: M\}$ gated by the track $\tau \in \mathcal{T}$. 
Table 6.1: An example of a gating matrix for two measurements and three tracks, where the symbol $\checkmark$ indicates that the measurement is within the gate of the track.

\begin{tabular}{clc}
\hline & \multicolumn{2}{c}{ Measurements } \\
Tracks & $\mathbf{y}_{1}$ & $\mathbf{y}_{2}$ \\
\hline $\operatorname{Track} \tau_{1}$ & $\checkmark$ & \\
$\operatorname{Track} \tau_{2}$ & $\checkmark$ & $\checkmark$ \\
$\operatorname{Track} \tau_{3}$ & & $\checkmark$ \\
\hline
\end{tabular}

Table 6.2: An example of an association matrix for two measurements and three tracks, where each element contains the log probability contributed to the hypothesis score if the measurement is associated to the track. The bottom rows are for associations to false detections and new tracks. The symbol $\times$ indicates that association is not to be considered according to the gating matrix, corresponding to a contribution of negative infinity.

\begin{tabular}{rcc}
\hline & \multicolumn{2}{c}{ Measurements } \\
Assignments & $\mathbf{y}_{1}$ & $\mathbf{y}_{2}$ \\
\hline Track $\tau_{1}$ & $\log \frac{P_{D} p\left(\mathbf{y}_{1} \mid \tau_{1}\right)}{1-P_{D} P_{G}}$ & $\times$ \\
Track $\tau_{2}$ & $\log \frac{P_{D} p\left(\mathbf{y}_{1} \mid \tau_{2}\right)}{1-P_{D} P_{G}}$ & $\log \frac{P_{D} p\left(\mathbf{y}_{2} \mid \tau_{2}\right)}{1-P_{D} P_{G}}$ \\
Track $\tau_{3}$ & $\times$ & $\log \frac{P_{D} p\left(\mathbf{y}_{2} \mid \tau_{3}\right)}{1-P_{D} P_{G}}$ \\
& $\log \left(\beta_{f}\right)$ & $\times$ \\
False Detections & $\times$ & $\log \left(\beta_{f}\right)$ \\
& $\log \left(\beta_{n}\right)$ & $\times$ \\
New Tracks & $\times$ & $\log \left(\beta_{n}\right)$ \\
& &
\end{tabular}

Similarly, the association matrix A has one measurement per column and one track per row, with additional rows corresponding to false detections and new tracks. An example of an association matrix is shown in Table 6.2.

An assignment can be represented as a matrix $\mathbf{M}$ of dimension $(N+2 M) \times$ $M$, where elements are either zero or one. The matrix has exactly one nonzero element per column, $\sum_{l} \mathbf{M}_{l, i}=1 \forall i$, and at most one non-zero element per row, $\sum_{i} \mathbf{M}_{l, i} \leq 1 \forall l$, where $\mathbf{A}_{a, b}$ denotes the element at row $a$ and column $b$. The motivation for this structure is the assumptions that each measurement is generated by at most one target and that each target generates at most one measurement. An equivalent and more compact representation is a vector $\mathbf{m}$ of dimension $M$ where each element corresponds to a measurement and points to the associated track, a false detection or a new track.

The assignment problem, formulated using the matrix representation, is then defined as

$$
\underset{\mathbf{M}}{\operatorname{minimize}} \quad \sum_{l} \sum_{i} \mathbf{M}_{l, i} \mathbf{A}_{l, i}
$$




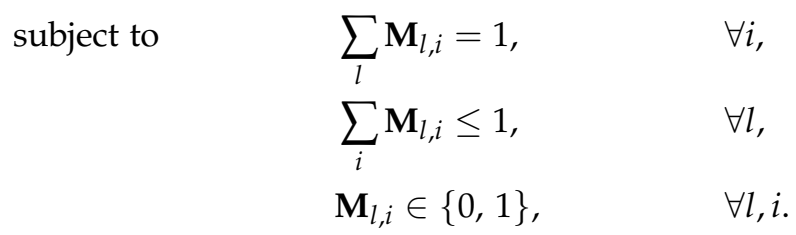

There are many algorithms available to solve this problem, e.g., the hungarian method [109], Munkres algorithm [30], Jonker-Volgenant relaxation [97], the auction algorithm [24, 25, 41] and the Jonker-Volgenant-Castanón algorithm [55]. The auction algorithm is considered the most efficient in [31]. In [113] it is stated that the auction algorithm is never faster and often much slower than the Jonker-Volgenant-Castanón algorithm, but is praised for being both efficient and intuitive. To find a number of the best solutions to the assignment problem Murty's method can be used [48, 49, 134].

\subsubsection{Coarse Gating and Clustering}

For scenarios with a large number of tracks and measurements, the complexity of the assignment problem becomes prohibitively large for real-time implementation. Ellipsoidal gating also becomes expensive due to the computation of the Mahalanobis distance between all combinations of tracks and measurements.

To reduce the number of ellipsoidal gates it is proposed that coarse gates are used. The gate in (6.4) is rewritten for each track by defining a region as the set

$$
\left\{\mathbf{y}:\left(\mathbf{y}-\hat{\mathbf{y}}^{\tau}\right)^{\top}\left(\mathbf{S}^{\tau}\right)^{-1}\left(\mathbf{y}-\hat{\mathbf{y}}^{\tau}\right)<\gamma\right\}
$$

to allow for more efficient approaches. One such approach is the following. First, the axis-aligned bounding boxes of the gate region for each target in $\mathcal{T}$ are computed or conservatively approximated. The bounding boxes are then inserted into a data structure suitable for spatial indexing, such as an R-tree [83], from which candidate targets are quickly obtained for each measurement. Ellipsoidal gates can then be applied to further reduce the set of targets.

Further, groups of targets that share gated measurements, directly or indirectly, can be clustered together. By viewing the gating matrix as an adjacency matrix for a bipartite graph, clusters are constructed using, e.g., the connected component algorithm [56, 170]. The association matrix, see Table 6.2, can then be permuted into a block diagonal matrix with one block for each cluster. The advantage of this is that the assignment problem can be solved separately for each cluster. The complexity of finding the $K$-best assignments is $\mathcal{O}\left(K(M+2 N)^{4}\right)$ [188] using Murty's algorithm [134], which can be reduced to $\mathcal{O}\left(K(M+2 N)^{3}\right)$ [188] using more efficient algorithms [129, 146]. Clustering the targets and measurements therefore results in a significant reduction in computation time. 


\subsection{Multiple Hypothesis Tracker}

The MHT, described in e.g. [31], is a common algorithm for multiple target tracking (Мтт). The tracker is complete in its non-approximated form in the sense that all hypotheses are explored. However, the tracker is intractable due to the combinatorial explosion of the number of hypotheses to consider, which grows exponentially in time. One common approach for handling the intractability is to reduce the number of maintained hypotheses, through pruning of lowprobability hypotheses and merging of similar hypotheses [31]. The GNN tracker is an important special case that is obtained when only the most probable hypothesis is maintained in each iteration [31].

There are two common approaches to MHT [31], hypothesis oriented and track oriented. In the former, a number of the most likely hypotheses are maintained at each time step, where each hypothesis consists of its own set of tracks. This is the original approach, presented in [153], and it is relatively simple to implement. The main drawback, however, is that multiple copies of identical tracks might be maintained in different hypotheses. The latter instead maintains a set of distinct tracks and constructs hypotheses at each time step of non-conflicting tracks [31, 196].

The MHT presented here is hypothesis oriented, where a set of the $N_{H}$ most likely hypotheses are maintained. At the arrival of new measurements, the $N_{H}$ new most likely hypotheses are constructed from each maintained hypothesis, resulting in $N_{H}^{2}$ hypotheses. Of these, the $N_{H}$ most likely hypotheses are maintained for the next time step. The algorithm for the tracker is outlined in Algorithm 6.1. A hypothesis is represented by a set of tracks and a probability. Given a hypothesis and associated measurements, estimation in the tracker is performed using the time update and measurement update steps in, e.g., the KF, EKF or PF. To reduce the number of considered associations, a gating matrix is constructed for each hypothesis as described in Section 6.1.4. Given the predicted tracks, the measurements and the gating matrix an association matrix is computed for each hypothesis as described in Section 6.1.4. The assignment problem is then solved for each hypothesis using Murty's algorithm to find a number of the most likely new hypotheses and their probabilities, as discussed in Section 6.1.4.

The MHт is used in the savannah application and the results are shown in Application 6.1.

\section{Application 6.1: Savannah Animal Tracking}

Savannah animals are tracked in image coordinates as a quadcopter flies above the savannah at Kolmården Wildlife Park. The model used for the animals is described in Application 3.6, but the origins of the measurements are uncertain. To handle the uncertainty the MHT is applied as outlined in Algorithm 6.1, with the time and measurement updates replaced by (5.36) and (5.37), respectively. Figure 6.2 shows an outline of the elements included in the tracker. $M / N$-logic is used for deletion of tracks and Murty's algorithm with the auction algorithm is used to solve the assignment problem. 


\section{Algorithm 6.1: Multiple Hypothesis Tracker}

Input: $\mathcal{Y}_{k}$ for $k \in\{1: N\}$

Initialization

$\mathcal{T}_{0} \leftarrow\left\{\mathcal{T}_{0}^{i}\right\}_{i=1}^{N_{H}}$, where $\mathcal{T}_{0}^{i}=\varnothing, \forall i \in\left\{1: N_{H}\right\}$

$\mathcal{P}_{0} \leftarrow\left\{p_{0}^{i}\right\}_{i=1}^{N_{H}}$, where $p_{0}^{1}=1$ and $p_{0}^{i}=0, \forall i \in\left\{2: N_{H}\right\}$

for $k=1, \ldots, N$ do

Time Update and Data Association

for $i=1, \ldots, N_{H}$ do

$\mathcal{T}_{+}^{i} \leftarrow$ Time Update $\left(\mathcal{T}_{k-1}^{i}\right)$

$\mathrm{G}^{i} \leftarrow$ Gating Matrix $\left(\mathcal{T}_{+}^{i}, \mathcal{Y}_{k}\right)$

$\mathbf{A}^{i} \leftarrow$ Association $\operatorname{Matrix}\left(\mathcal{T}_{+}^{i}, \mathcal{Y}_{k}, \mathbf{G}^{i}\right)$

$\left\{\mathbf{m}^{i, j}, p^{i, j}\right\} \leftarrow$ Solve Assignment Problem $\left(\mathbf{A}^{i}, p_{k-1}^{i}, N_{H}\right)$

end for

Pruning and Measurement Update

$\left\{i_{n}, j_{n}\right\} \leftarrow$ Most Likely Hypotheses $\left(\left\{p^{i, j}\right\}, N_{H}\right)$

for $n=1, \ldots, N_{H}$ do

$p_{k}^{n} \leftarrow p^{i_{n}, i_{n}}$

$\mathcal{T}_{k}^{n} \leftarrow$ Measurement $\operatorname{Update}\left(\mathcal{T}_{+}^{i_{n}}, \mathcal{Y}_{k}, \mathbf{m}^{i_{n}, j_{n}}\right)$

end for

$\mathcal{T}_{k} \leftarrow\left\{\mathcal{T}_{k}^{n}\right\}_{n=1}^{N_{H}}$

end for

$\mathcal{P}_{k} \leftarrow \operatorname{Normalize}\left(\left\{p_{k}^{n}\right\}_{n=1}^{N_{H}}\right)$

output: $\mathcal{T}_{k}$ and $\mathcal{P}_{k}$ for $k \in\{1: N\}$

An example of the results from the MHT is shown in Figure 6.3. A few targets are unresolved in the sense that one track corresponds to multiple targets. This issue results from the measurement pre-processing and is not corrected by the tracker.

Extending the algorithm to tracking in world coordinates results in several additional challenges and would for example require:

- a three-dimensional extension model;

- a ground model for relatively accurate position estimates of the animals;

- identification and classification of the animals to reduce data association uncertainty;

- calibration of the camera;

- estimation of the camera position and orientation; and

- consideration of occlusions from the terrain and vegetation.

The practical use of MHT and ETT on real data has been demonstrated, concluding the savannah application. 


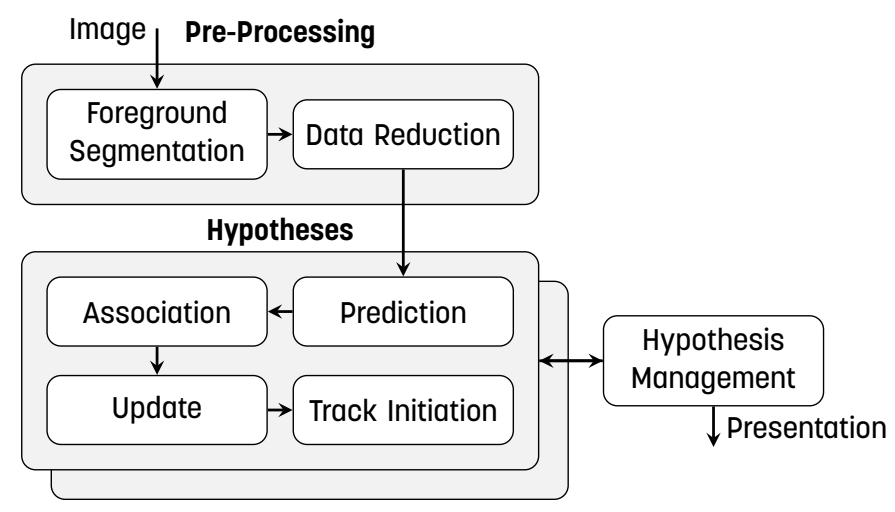

Figure 6.2: The pipeline of processing a frame from the sensor in the savannah application.

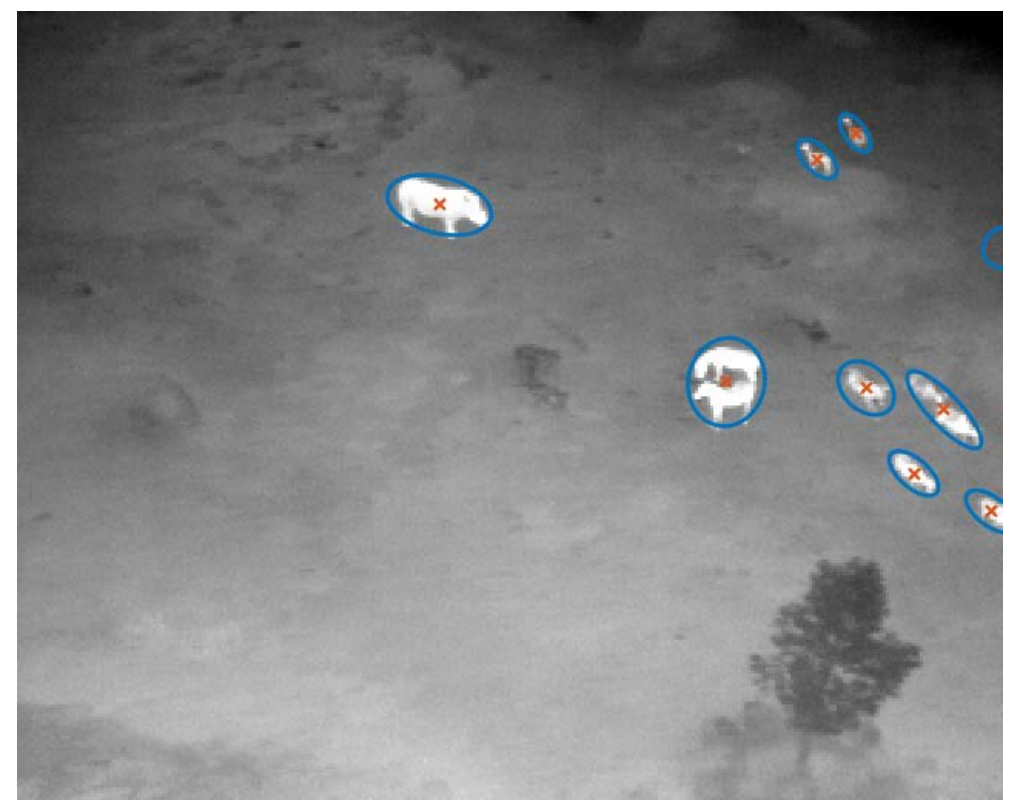

Figure 6.3: An example of the results from the MHT. The red cross shows the position of the target and the blue ellipse shows the estimated extent based on a uniform measurement distribution. 


\subsection{Probabilistic Data Association Filter}

In applications with few targets, high probability of detection and few false detections less demanding filters may achieve similar tracking performance as the мнт. One such single target tracking (STT) filter is the PDA filter, originally presented in [14]. The approach is to consider multiple hypotheses for association of gated measurements to each track and merge them into a single hypothesis at each time step, as described in [11,31]. Note that, unlike the association hypotheses described in Section 6.1 that consider all tracks, these association hypotheses are local and independent for each track. An MTT extension to the filter, the JPDA filter [11, 64], is designed for improved tracking performance for scenarios with multiple targets.

The conventional PDA filter is described in Section 6.3.1. Track management for the PDA filter is described in Section 6.3.2 as well as a proposed method for merging similar tracks. Section 6.3 .3 presents a modification of the PDA filter, first published by the author in [181], that allows for estimation of the target extent.

\subsubsection{Standard Probabilistic Data Association Filter}

In this section the PDA filter is described briefly. See [31] for a more thorough description. Given is a set $\mathcal{T}$ of $N$ tracks and a set $\mathcal{Y}=\left\{\mathbf{y}_{i}\right\}_{i=1}^{M}$ of new measurements. The predicted distributions of the state and measurement for a track $\tau \in \mathcal{T}$ is given by $p(\mathbf{x} \mid \tau)=\mathcal{N}\left(\mathbf{x} \mid \hat{\mathbf{x}}^{\tau}, \mathbf{P}^{\tau}\right)$ and $p(\mathbf{y} \mid \tau)=\mathcal{N}\left(\mathbf{y} \mid \hat{\mathbf{y}}^{\tau}, \mathbf{S}^{\tau}\right)$, respectively. Note that the time index is omitted for notational convenience.

Gating is first performed as described in Section 6.1.1 for the measurements $\mathcal{Y}$ and tracks $\mathcal{T}$. This results in the sets $\mathcal{G}_{\tau}$ defined in Section 6.1.4. The number of measurements gated to track $\tau$ is given by $M_{\tau}=\left|\mathcal{G}_{\tau}\right|$. For each track $\tau \in \mathcal{T}$, hypotheses $\theta_{\tau}^{i}$ are constructed for all $i \in \mathcal{G}_{\tau}$. Hypothesis $\theta_{\tau}^{i}$ states that the gated measurement $\mathbf{y}_{i} \in \mathcal{Y}$ is generated by the target and all other measurements in the gate are false detections. The probability of such a hypothesis for the LGSSM is

$$
\begin{aligned}
\mu_{i}^{\tau} \triangleq p\left(\theta_{\tau}^{i} \mid \mathcal{Y}, \tau\right) & \propto \beta^{M_{\tau}-1} P_{D} p\left(\mathbf{y}_{i} \mid \theta_{i}^{\tau}, \tau\right) \\
& =\beta^{M_{\tau}-1} P_{D} \mathcal{N}\left(\mathbf{y}_{i} \mid \hat{\mathbf{y}}^{\tau}, \mathbf{S}^{\tau}\right), \quad i \in \mathcal{G}_{\tau},
\end{aligned}
$$

where $\beta=\beta_{f}+\beta_{n}$. In addition, a hypothesis $\theta_{\tau}^{0}$ is constructed that all measurements in the gate are false detections, with probability

$$
\mu_{0}^{\tau}=p\left(\theta_{0}^{\tau} \mid \mathcal{Y}, \tau\right) \propto \beta^{M_{\tau}}\left(1-P_{D} P_{G}\right) .
$$

The hypothesis probabilities $\mu_{i}^{\tau}$ are normalized over $i \in \mathcal{G}_{\tau} \cup 0$. The posterior distribution of the state for each track $\tau \in \mathcal{T}$ is

$$
p(\mathbf{x} \mid \tau, \mathcal{Y})=\sum_{i \in \mathcal{\mathcal { G } _ { \tau }} \cup 0} p\left(\mathbf{x}, \theta_{i}^{\tau} \mid \tau, \mathcal{Y}\right)=\sum_{i \in \mathcal{G} \tau} p\left(\theta_{i}^{\tau} \mid \tau, \mathcal{Y}\right) p\left(\mathbf{x} \mid \theta_{i}^{\tau}, \tau, \mathcal{Y}\right)
$$




$$
=\sum_{i \in \mathcal{G}_{\tau} \cup 0} \mu_{i}^{\tau} \mathcal{N}\left(\mathbf{x} \mid \hat{\mathbf{x}}_{i}^{\tau}, \mathbf{P}_{i}^{\tau}\right),
$$

which is a mixture of Gaussians. The last factor, $\mathcal{N}\left(\mathbf{x} \mid \hat{\mathbf{x}}_{i}^{\tau}, \mathbf{P}_{i}^{\tau}\right)$, is obtained by performing a measurement update for the track $\tau$ in the KF in Algorithm 5.2 with the associated measurement $\mathbf{y}_{i}$. For the hypothesis $\theta_{0}^{\tau}$ the prediction distribution for the track is used. The merged hypothesis is approximated as a Gaussian by matching the first two moments of the distribution, resulting in

$$
p(\mathbf{x} \mid \tau, \mathcal{Y}) \approx \mathcal{N}\left(\mathbf{x} \mid \hat{\mathbf{x}}_{+}^{\tau}, \mathbf{P}_{+}^{\tau}\right)
$$

where

$$
\begin{aligned}
\hat{\mathbf{x}}_{+}^{\tau} & =\sum_{i \in \mathcal{G}_{\tau} \cup 0} \mu_{i}^{\tau} \hat{\mathbf{x}}_{i}^{\tau}, \\
\mathbf{P}_{+}^{\tau} & =\sum_{i \in \mathcal{G}_{\tau} \cup 0} \mu_{i}^{\tau}\left(\mathbf{P}_{i}^{\tau}+\left(\hat{\mathbf{x}}_{i}^{\tau}-\hat{\mathbf{x}}_{+}^{\tau}\right)\left(\hat{\mathbf{x}}_{i}^{\tau}-\hat{\mathbf{x}}_{+}^{\tau}\right)^{\top}\right) .
\end{aligned}
$$

\subsubsection{Track Management}

To initiate new tracks, confirmed and tentative tracks are treated separately. The steps in Section 6.3.1 are performed in two levels. The first level is for all confirmed tracks and the second level is for all tentative tracks using the non-gated measurements. Any remaining non-gated measurements are considered to be false detections or new tracks. These are used to initiate new tentative tracks. Tentative tracks are either confirmed or deleted based on the track quality as described in Section 6.1.3.

Track management also includes merging similar tracks. The motivation for merging similar tracks is to improve the performance of the PDA filter. One such method is described in this section. Similar tracks $\tau_{a}$ and $\tau_{b}$ are merged based on the Bhattacharyya distance [26]

$$
\begin{aligned}
d_{B}\left(\tau_{a}, \tau_{b}\right)=\frac{1}{4}\left(\hat{\mathbf{x}}^{\tau_{a}}-\hat{\mathbf{x}}^{\tau_{b}}\right)^{\top}\left(\mathbf{P}^{\tau_{a}}+\mathbf{P}^{\tau_{b}}\right)^{-1}\left(\hat{\mathbf{x}}^{\tau_{a}}-\hat{\mathbf{x}}^{\tau_{b}}\right) \\
+\frac{1}{2} \ln \left(\frac{\left|\left(\mathbf{P}^{\tau_{a}}+\mathbf{P}^{\tau_{b}}\right) / 2\right|}{\sqrt{\left|\mathbf{P}^{\tau_{a}}\right|\left|\mathbf{P}^{\tau_{b}}\right|}}\right),
\end{aligned}
$$

which measures the similarity between two multivariate normal distributions. If a subset of tracks $\mathcal{T}^{\prime} \subseteq \mathcal{T}$ satisfies $d_{B}\left(\tau_{a}, \tau_{b}\right) \leq \gamma_{m}$ for all $\tau_{a}, \tau_{b} \in \mathcal{T}^{\prime}$, where $\gamma_{m}$ is a design parameter, the tracks are merged. Tracks are merged using [158]

$$
\begin{aligned}
\hat{\mathbf{x}}^{+} & =\sum_{\tau \in \mathcal{T}^{\prime}} w_{\tau} \hat{\mathbf{x}}^{\tau}, \\
\mathbf{P}^{+} & =\sum_{\tau \in \mathcal{T}^{\prime}} w_{\tau}\left(\mathbf{P}^{\tau}+\left(\hat{\mathbf{x}}^{\tau}-\hat{\mathbf{x}}^{+}\right)\left(\hat{\mathbf{x}}^{\tau}-\hat{\mathbf{x}}^{+}\right)^{\top}\right),
\end{aligned}
$$

where the weight $w_{\tau}=\left|\mathbf{P}^{\tau}\right| / \sum_{\tau^{\prime} \in \mathcal{T}^{\prime}}\left|\mathbf{P}^{\tau^{\prime}}\right|$ is chosen to prioritize tracks with a large extent. The posterior distribution of the track with the largest weight is replaced by $\mathcal{N}\left(\hat{\mathbf{x}}^{+}, \mathbf{P}^{+}\right)$and the remaining tracks are deleted. 


\subsubsection{Modified Probabilistic Data Association Filter}

A modification of the PDA filter, that was first published by the author in [181], is presented in this section. A side effect of the PDA filter when receiving many measurements with high covariance near a track is that the state covariance grows, and consequently also the covariance of the measurement prediction. Therefore, by a certain abuse of the filter, an estimate of the extent of the target can be obtained from the measurement distribution of the track without explicitly modelling the extent.

By considering the reduced and mapped measurements in (4.23), the PDA filter can be modified to estimate the extent and improve tracking performance in certain scenarios. The measurement triplet

$$
\mathcal{Y}=\left\{\left(\mathbf{y}_{i}, d_{i}, \mathbf{Y}_{i}\right)\right\}_{i=1}^{M}
$$

restated here for convenience, consists of the mean $\mathbf{y}_{i}$ and covariance $\mathbf{Y}_{i}$ of the pixel coordinates as well as the mean degree of confidence $d_{i}$. Each triplet in the set corresponds to a number of segmented foreground pixels, which can be seen as individual detections of the target. The number of individual measurements represented by each mapped reduced measurement can be approximated by the determinant of the covariance matrix

$$
n_{i}=\left|\mathbf{Y}_{i}\right|,
$$

or by the determinant weighted by the degree of confidence, as defined in (4.17),

$$
n_{i}=d_{i}\left|\mathbf{Y}_{i}\right| .
$$

The determinant is proportional to the size of a confidence ellipsoid representing the corresponding Gaussian distribution. Each gated measurement $\mathbf{y}_{i}$ for track $\tau \in \mathcal{T}$, such that $i \in \mathcal{G}_{\tau}$, is used to update the PDA filter $n_{i}$ times, which results in the weights, with $n_{0}=1$,

$$
\mu_{i}^{\tau} \propto n_{i} p\left(\theta_{i}^{\tau} \mid \mathcal{Y}, \tau\right), \quad i \in \mathcal{G}_{\tau} \cup 0 .
$$

As argued in Section 5.5 a uniform distribution is a more reasonable assumption compared to a Gaussian distribution for the measurements in a camera sensor. The hypothesis probabilities in (6.15) are modified such that true measurements are uniformly distributed within the gate, resulting in

$$
p\left(\theta_{i}^{\tau} \mid \mathcal{Y}, \tau\right) \propto \frac{\beta^{M_{\tau}-1} P_{D}}{V}=\frac{\beta^{M_{\tau}-1} P_{D}}{\pi\left|4 \mathbf{S}^{\tau}\right|}, \quad i \in \mathcal{G}_{\tau},
$$

where $V$ is the volume of the gate and Proposition 5.3 is used.When the number of gated measurements $M_{\tau}$ is large, the probability $\mu_{0}^{\tau}$ of hypothesis $\theta_{0}^{\tau}$ approaches zero. The weights can then be approximated by

$$
\mu_{i}^{\tau} \approx \frac{n_{i}}{\sum_{i \in \mathcal{G}_{\tau}} n_{i}}, \quad \quad i \in \mathcal{G}_{\tau} .
$$




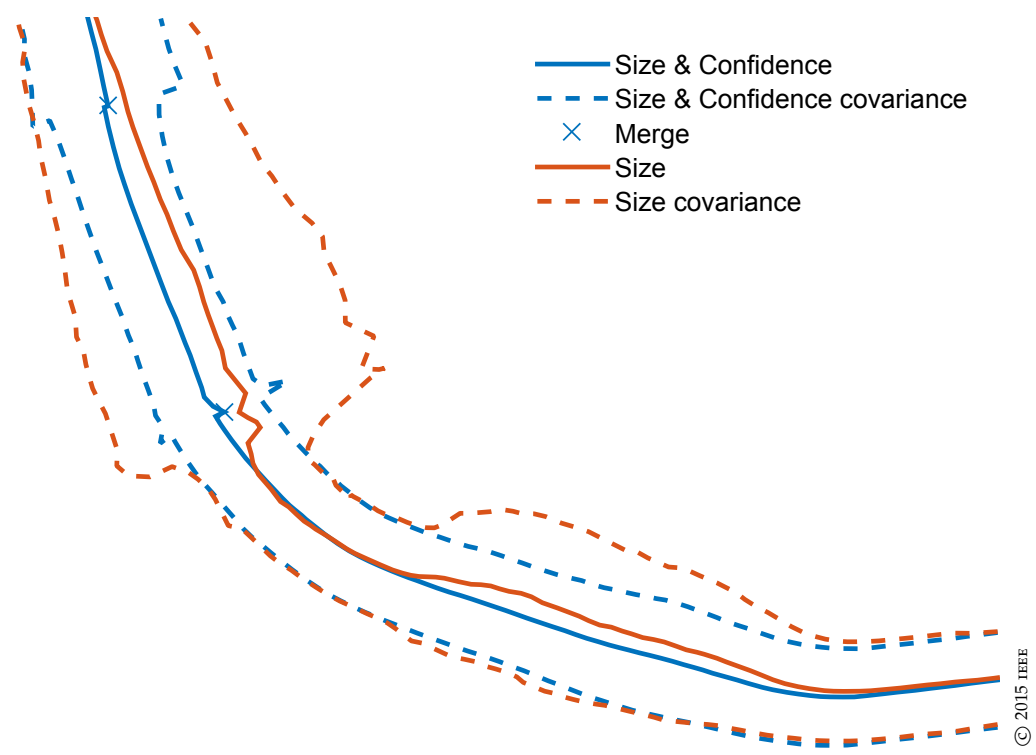

Figure 6.4: Compares the estimated trajectory and the estimated extent in the presence of a shadow using size and degree of confidence as well as only size as multiplicity of the measurements. The estimated extent for the former trajectory is less sensitive to the shadow. Published in [181].

Application 6.2 compares the standard and modified PDA filters for the dolphin application as well as the different multiplicities in (6.23) and (6.24). The comparison was previously published by the author in [181].

\section{Application 6.2: Dolphin Filter Comparison}

The PDA filter is compared to the modified PDA filter proposed in this section for the dolphin application. Also, the two different multiplicities in (6.23) and (6.24) are compared for the modified PDA filter.

Targets cast shadows, as seen in Figure 4.5, which often have a lower degree of confidence than the targets. Using only size as in (6.23) to determine the measurement multiplicity, all foreground measurements will have similar weights in the target tracking filter, while including the degree of confidence as in (6.24) puts higher weights on true targets than shadows. To compare the two options the results in the presence of the shadow in Figure 4.5a is shown in Figure 6.4. Using only size, the trajectory is sensitive to the shadow, since the same weight is put on all measurements. However, when using both size and degree of confidence in the multiplicity the shadow measurements have less impact. This gives a smoother trajectory estimate and an innovation covariance that mainly covers the target. The side effect is that a new track is initiated on the shadow, which is subsequently merged into the original track with little effect to its trajectory.

For the PDA filter described in Section 6.3 the measurements are assumed to follow a Gaussian distribution around the target position, effectively giving 


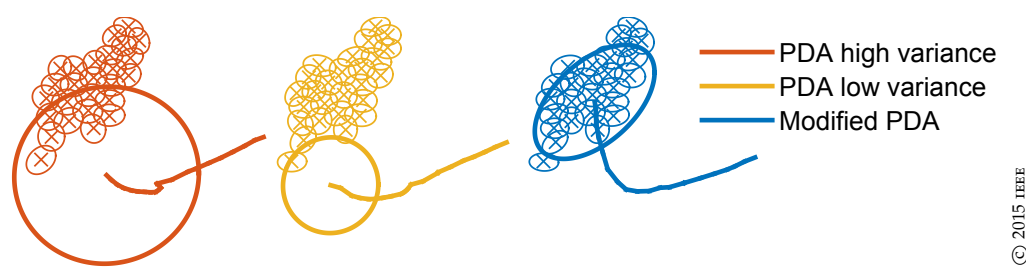

Figure 6.5: Comparison of the estimated centroid and extent for the standard PDA filter, using high and low Gaussian measurement noise covariance, with the modified PDA filter during a sharp turn. Published in [181].

more weight to measurements near the centroid. This results in poor estimation of the target extent. To mitigate this effect, the modified PDA filter in Section 6.3.3 is used instead. It assumes uniformly distributed measurements in the gate as proposed in (6.26) and utilizes the multiplicity of the measurements as in (6.25). To compare the two options the filter performances are evaluated in a sharp turn. The results are given in Figure 6.5. The standard PDA filters struggle to track the target through the turn for various choices of process and measurement noise covariances, while the modified PDA filter finds the centroid. It also adapts the covariance to match the extent of the target, improving the performance.

The proposed modifications in the modified PDA filter are relatively minor, but are shown to perform better in certain scenarios. A noticeable problem with the proposed method is that the extent estimate grows with missed detections, since the state covariance is reused. This could be mitigated by using an explicit extent model, e.g., as described in Section 3.7. Similar modifications to handle uniformly distributed measurements and degree of confidence could be applied for this model as well. See Application 6.3 for a presentation of the full target tracking framework and the results.

In Application 6.3 the full framework is put together for tracking in the dolphin application. A discussion regarding the results is presented. The framework and the results were first published by the author in [181].

\section{Application 6.3: Dolphin Framework}

The full framework for tracking the dolphins is put together. The pre-processing of the measurements, as presented in Application 4.3 for foreground segmentation and in Applications 4.2 and 4.8 for mapping and measurement reduction, is utilized. The ssm considered is presented in Application 3.2.

Detecting and tracking the dolphins comes with many uncertainties, e.g., number of targets, false detections, missed detections, unresolvable targets and association uncertainty among others. A few methods to handle these uncertainties are discussed in this chapter. In Section 4.4 each dolphin generates a number of detections, while the assumption for the estimation is that the target can generate at most one detection at each time step. This is utilized in the modified PDA filter. 


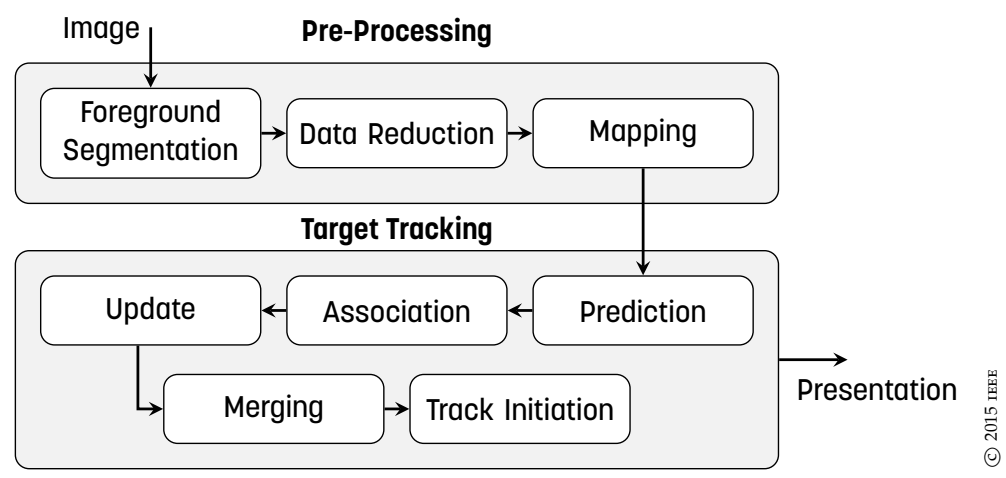

Figure 6.6: The pipeline of processing a frame from the sensor in the dolphin application. Track Initiation essentially consists of an additional target tracking module for tentative tracks. Published in [181].

The motion model of the dolphin is given by (3.39) and (3.31) and the sensor model is given by (3.44). The target states are initialized as described for the NCVM in Section 3.4. The EKF in Algorithm 5.2 is applied to the sequence of measurements using these models. The measurement update step is omitted when no measurement is available. The linearization around the state of the motion model is obtained from (3.41). To handle the data association uncertainty, the modified PDA filter presented in this section is used with track management as described in Section 6.3.2. The measurement set in Application 4.8 is used in the filter, and (6.24) is used as multiplicity. Figure 6.6 shows an outline of the elements included in the tracker.

The trajectory resulting from the filter for one group of dolphins is shown in Figure 6.7. On several occasions tracks are merged along the way. Although not visible in the figure, several individuals also leave the group along the trajectory, initiating new tracks. The advantage of the СмM is apparent at the bottom left where the target is occluded for over 100 frames while the track is maintained. It is apparent from the mapped detection region that the mapping is not completely accurate. This is a result of an inaccurate structure for the camera model or issues related to the estimation of the camera model.

The framework is applied to five minute sequences recorded at Kolmården Wildlife Park and outputs a set of trajectories. The main difficulty is the handling of dolphins entering and leaving groups, which provides difficulties for the tracking algorithm as well as for the presentation of the results. An alternative to presenting individual tracks is to aggregate all estimated tracks into a map to show where the dolphins spend most time. Figure 6.8 shows such a map where preferred regions and trajectories are clearly visible. This could be presented to the biologists as a supplement to the individual trajectories for a quick overview. The practical use of the CMM and the modified PDA filter on real data has been demonstrated, concluding the dolphin application. 


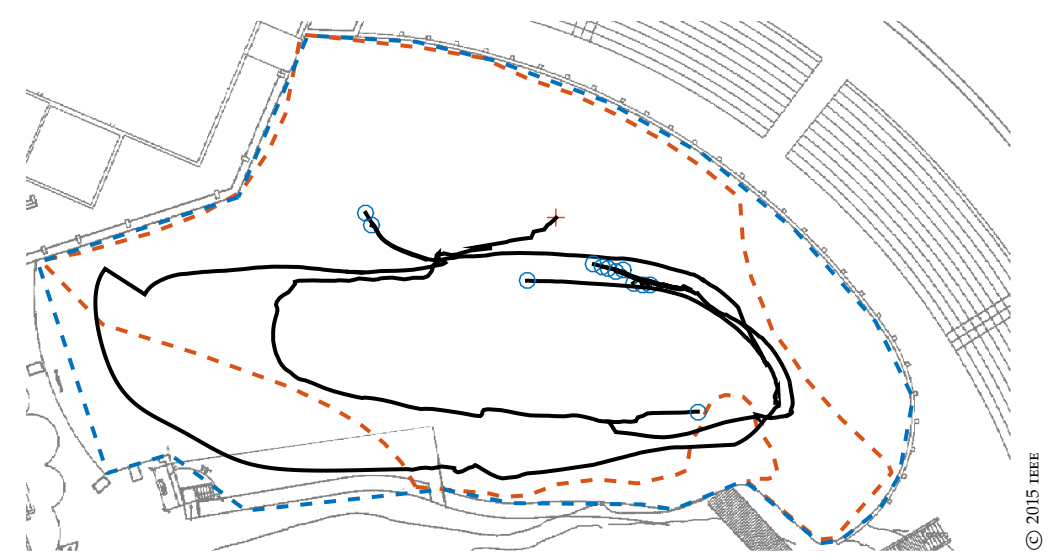

Figure 6.7: A track of a group of targets, with individuals joining and splitting from the group. The blue circles show initiated tracks that are merged with the long track. The dashed blue line shows the constraint region and the dashed red line shows the mapped detection region. The filter succeeds in maintaining the track while the targets move outside of the detection region at the bottom right and when the target disappears for a long time in the non-detection region at the bottom left. Published in [181].

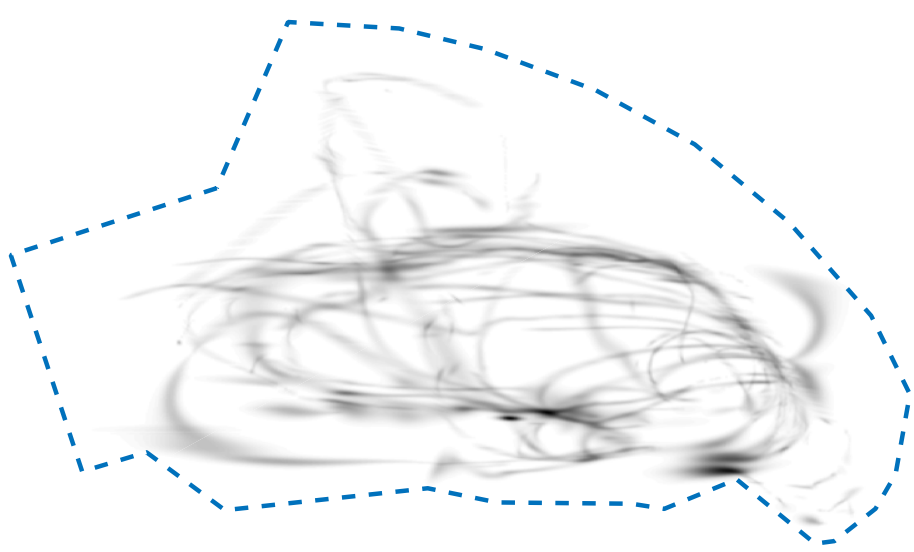

Figure 6.8: A map showing the aggregated estimates of all dolphins for a five minute sequence. Although individual tracks are discarded, the behaviour of the dolphins can be analysed from such presentations. The dashed blue line shows the constraint region. 


\subsection{Labelled Multi-Bernoulli Filter}

Traditionally multi-target tracking is decomposed into the two subproblems of data association and filtering. In the last decade a contending method to classical target tracking has emerged in the framework of finite set statistics (FISST) [121]. In this approach both the multi-target states and the measurements are modelled as random finite sets (RFS). An RFS is a finite unordered set for which the number of elements is random and the elements are random vectors. This leads to a recursive Bayesian formulation of MTT in the form of the multi-target Bayes filter [120]. The main advantage is that both data association and filtering are expressed in a single theoretic framework with a single equation each for prediction and measurement update [36]. The MHT filter [153] can be derived from FISST under weak assumptions [36], showing a close relationship between the two approaches.

The generalized labelled multi-Bernoulli (GLMB) RFs proposed in [189] is of particular interest, since its PDF can be seen as a mixture of multi-target state distributions. Each component in the mixture represents a particular hypothesis of the multi-target state. It is shown in [187] to result in an exact analytic solution of the multi-target Bayes filter. The resulting $\delta$-GLMB filter [187-189] resembles the MHT filter in many ways and has similar computational complexity [119]. The LмB filter is proposed in [154] as a fast approximation. The general idea is to collapse the resulting $\delta$-GLMB RFS in each iteration into an LMB RFS, merging all hypotheses into one hypothesis. The resulting filter shows a resemblance to the classical JPDA filter [31] in the sense that they both are MTT filters that reduce the complexity by merging hypotheses.

The remainder of this section outlines the Lмв filter and proposes a reformulation of the original LMB filter that improves computational efficiency. Note that the terminology in this section differs from other parts of the thesis to match the notation of the original papers.

\subsubsection{Labelled Multi-Bernoulli Filter}

The LMB filter is defined in the FISST framework [154], of which the RFS is an integral part. An RFS is a set with a probabilistic cardinality, i.e., each potential element is included in the set with a given probability. Specifically, a Bernoulli RFS is a random set which is empty with probability $1-r$, and with probability $r$ is a singleton. For an element $\mathbf{x} \in \mathbb{R}^{n}$ with probability $p(\mathbf{x})$, the Bernoulli RFS PDF is given by

$$
p(\mathcal{X})= \begin{cases}1-r, & \text { if } \mathcal{X}=\varnothing \\ r \cdot p(\mathbf{x}), & \text { if } \mathcal{X}=\{\mathbf{x}\}\end{cases}
$$

A multi-Bernoulli RFS is the resulting set of the union of $N$ independent Bernoullidistributed RFS $\mathcal{X}^{(i)}$, given by $\mathcal{X}=\bigcup_{i=1}^{N} \mathcal{X}^{(i)}$. Consequently, the multi-Bernoulli 
RFS is parametrized by the set $\left\{\left(r^{(i)}, p^{(i)}\right)\right\}_{i=1}^{N}$, and its PDF is given by [121]

$$
p\left(\left\{\mathbf{x}_{1}, \ldots, \mathbf{x}_{n}\right\}\right)=\prod_{j=1}^{N}\left(1-r^{(j)}\right) \times \sum_{1 \leq i_{1} \neq \cdots \neq i_{n} \leq N} \prod_{j=1}^{n} \frac{r^{\left(i_{j}\right)} p^{\left(i_{j}\right)}\left(\mathbf{x}_{j}\right)}{1-r^{\left(i_{j}\right)}} .
$$

The LMB RFS is obtained by the augmentation of each Bernoulli RFS with a unique label, $\ell \in \mathcal{L}$. The RFs can thus be described by the set

$$
\pi=\left\{\left(r^{(\ell)}, p^{(\ell)}\right)\right\}_{\ell \in \mathcal{L}^{\prime}}
$$

which fully describes the multi-target probability density $p(\mathcal{X})$. The LMB filter follows the classical time and measurement update filter recursion presented in Section 5.1 and is outlined below.

\section{Time Update}

Given a posterior distribution LMB PDF $p\left(\mathcal{X}_{-}\right)$the prediction distribution $p\left(\mathcal{X}_{+}\right)$ is obtained by the application of the standard prediction of a Bayesian filter, the Chapman-Kolmogorov equation,

$$
p\left(\mathcal{X}_{+}\right)=\int p\left(\mathcal{X}_{+} \mid \mathcal{X}_{-}\right) p\left(\mathcal{X}_{-}\right) \delta \mathcal{X}_{-}
$$

extended for an RFS as defined in [189], where $\mathcal{X}_{-}$and $\mathcal{X}_{+}$are shorthands for the posterior distribution $\mathcal{X}_{k-1} \mid \mathcal{Y}_{1: k-1}$ and the prediction distribution $\mathcal{X}_{k} \mid \mathcal{Y}_{1: k-1}$, respectively. This gives the following set of surviving and new-born targets [154],

$$
\begin{aligned}
\pi_{+} & =\pi_{S} \cup \pi_{B}=\left\{\left(r_{+, S^{\prime}}^{(\ell)}, p_{+, S}^{(\ell)}\right)\right\}_{\ell \in \mathcal{L}} \cup\left\{\left(r_{B}^{(\ell)}, p_{B}^{(\ell)}\right)\right\}_{\ell \in \mathcal{B}} \\
& =\left\{\left(r_{+}^{(\ell)}, p_{+}^{(\ell)}\right)\right\}_{\ell \in \mathcal{L}_{+}}{ }^{\prime}
\end{aligned}
$$

where

$$
\begin{aligned}
r_{+, S}^{(\ell)} & =\eta_{S}^{(\ell)} r^{(\ell)}, \\
p_{+, S}^{(\ell)}\left(\mathbf{x}_{+}\right) & =\frac{\int p_{S}^{(\ell)}(\mathbf{x}) p_{+}^{(\ell)}\left(\mathbf{x}_{+} \mid \mathbf{x}\right) p^{(\ell)}(\mathbf{x}) d \mathbf{x}}{\eta_{S}^{(\ell)}}, \\
\eta_{S}^{(\ell)} & =\int p_{S}^{(\ell)}(\mathbf{x}) p^{(\ell)}(\mathbf{x}) d \mathbf{x},
\end{aligned}
$$

$p_{S}^{(\ell)}(\mathbf{x})$ is the distribution of target survival probability and $p_{+}^{(\ell)}\left(\mathbf{x}_{+} \mid \mathbf{x}\right)$ is the transition density. The set $\pi_{B}$ is given by the birth model, further discussed in Section 6.4.2. 


\section{Measurement Update}

Drawn from the update of the $\delta$-GLMв [189], the Lмв measurement update for the measurements $\mathcal{Y}$ is derived in [154]. In general, as noted in [154], the LMB distribution is not closed under the Bayesian filter update. However, the resulting $\delta$-GLMB distribution, which can represent multiple disjoint hypotheses, may be approximated with an LMB PDF through the collapse of its hypotheses, weighted by their probabilities. That is, the measurements update the set $\pi_{+}$in (6.32) by the approximation

$$
\pi\left(\mathcal{X}_{+} \mid \mathcal{Y}\right) \approx\left\{\left(r^{(\ell)}, p^{(\ell)}\right)\right\}_{\ell \in \mathcal{L}_{+}}
$$

in which the parameters are given by

$$
\begin{aligned}
& r^{(\ell)}=\sum_{\mathcal{I}_{+} \in \mathcal{F}\left(\mathcal{L}_{+}\right)} 1_{\mathcal{I}_{+}}(\ell) \sum_{\theta \in \Theta_{\mathcal{I}_{+}}} w_{\mathcal{Y}}^{\left(\mathcal{I}_{+}, \theta\right)}, \\
& p^{(\ell)}(\mathbf{x})=\frac{1}{r^{(\ell)}} \sum_{\mathcal{I}_{+} \in \mathcal{F}\left(\mathcal{L}_{+}\right)} 1_{\mathcal{I}_{+}}(\ell) \sum_{\theta \in \Theta_{\mathcal{I}_{+}}} w_{\mathcal{Y}}^{\left(\mathcal{I}_{+}, \theta\right)} \cdot p^{(\ell, \theta(\ell))}(\mathbf{x} \mid \mathcal{Y}),
\end{aligned}
$$

where $1_{\mathcal{B}}(\mathcal{A})$ is the inclusion function, defined as 1 if $\mathcal{B} \subseteq \mathcal{A}$ and 0 otherwise, $\mathcal{F}(\mathcal{A})$ is the collection of all subsets of $\mathcal{A}$ and $\Theta_{\mathcal{I}_{+}}$is the set of mappings of tracks $\theta: \mathcal{I}_{+} \rightarrow\{0:|\mathcal{Y}|\}$ such that $\theta(\ell)=\theta\left(\ell^{\prime}\right)>0$ implies that $\ell=\ell^{\prime}$, i.e., the mapping is unique for all values except those mapped to zero [154]. Also, for $\mathcal{I}_{+} \subseteq \mathcal{L}_{+}$

$$
\begin{aligned}
w_{\mathcal{Y}}^{\left(\mathcal{I}_{+}, \theta\right)} & \propto w_{+}^{\left(\mathcal{I}_{+}\right)} \prod_{\ell \in \mathcal{I}_{+}} \eta_{\mathcal{Y}}^{(\ell, \theta(\ell))}, \\
w_{+}^{\left(\mathcal{I}_{+}\right)} & =\prod_{\ell \in \mathcal{L}_{+}-\mathcal{I}_{+}}\left(1-r_{+}^{(\ell)}\right) \prod_{\ell^{\prime} \in \mathcal{I}_{+}} r_{+}^{\left(\ell^{\prime}\right)}, \\
\eta_{\mathcal{Y}}^{(\ell, i)} & =\int p_{+}^{(\ell)}(\mathbf{x}) \psi_{\mathcal{Y}}^{(\ell, i)}(\mathbf{x}) d \mathbf{x}, \\
\psi_{\mathcal{Y}}^{(\ell, i)}(\mathbf{x}) & = \begin{cases}\frac{p_{D}^{(\ell)}(\mathbf{x}) p_{+}^{(\ell)}\left(\mathbf{y}_{i} \mid \mathbf{x}\right)}{\kappa\left(\mathbf{y}_{i}\right)}, & i \neq 0, \\
1-p_{D}^{(\ell)}(\mathbf{x}) p_{G}, & i=0,\end{cases} \\
p^{(\ell, i)}(\mathbf{x} \mid \mathcal{Y}) & =\frac{p_{+}^{(\ell)}(\mathbf{x}) \psi_{\mathcal{Y}}^{(\ell, i)}(\mathbf{x})}{\eta_{\mathcal{Y}}^{(\ell, i)}},
\end{aligned}
$$

where $p_{G}$ is the gating probability, $p_{+}^{(\ell)}(\mathbf{y} \mid \mathbf{x})$ is the likelihood and $\kappa(\mathbf{y})$ is the Poisson clutter intensity [154]. Note that while the $\delta$-GLMB representation in [154] is an intermediate representation in the theoretical derivation of the filter, its construction in implementation is not necessary to reach the collapsed LMB representation of (6.34).

To calculate the weight from (6.36a) for a hypothesis a distinction is made between associated and non-associated targets by splitting the hypothesis label 
Table 6.3: An example of an association matrix for two measurements and three tracks, where each element contains the logarithm of the contribution to the hypothesis probability if the target is associated to the measurement. The rightmost columns are for associations to missed detections and non-existing targets. The symbol $\times$ indicates that association is not to be considered according to the gating matrix, corresponding to a contribution of negative infinity.

\begin{tabular}{ccccccccc}
\hline & \multicolumn{2}{c}{ Associated } & \multicolumn{3}{c}{ Missed } & \multicolumn{3}{c}{ Non-existing } \\
Target & $\mathbf{y}_{1}$ & $\mathbf{y}_{2}$ & & & & & \\
\hline Target $\tau_{1}$ & $\alpha^{\left(\tau_{1}, 1\right)}$ & $\times$ & $\mu^{\left(\tau_{1}\right)}$ & $\times$ & $\times$ & $v^{\left(\tau_{1}\right)}$ & $\times$ & $\times$ \\
Target $\tau_{2}$ & $\alpha^{\left(\tau_{2}, 1\right)}$ & $\alpha^{\left(\tau_{2}, 2\right)}$ & $\times$ & $\mu^{\left(\tau_{2}\right)}$ & $\times$ & $\times$ & $v^{\left(\tau_{2}\right)}$ & $\times$ \\
Target $\tau_{3}$ & $\times$ & $\alpha^{\left(\tau_{3}, 2\right)}$ & $\times$ & $\times$ & $\mu^{\left(\tau_{3}\right)}$ & $\times$ & $\times$ & $v^{\left(\tau_{3}\right)}$ \\
\hline
\end{tabular}

set $I_{+}$:

$$
\begin{aligned}
& \mathcal{I}_{+}^{a}=\left\{\ell \in \mathcal{I}_{+}: \theta(\ell) \neq 0\right\}, \\
& \mathcal{I}_{+}^{n}=\left\{\ell \in \mathcal{I}_{+}: \theta(\ell)=0\right\},
\end{aligned}
$$

implying $\mathcal{I}_{+}=\mathcal{I}_{+}^{a} \cup \mathcal{I}_{+}^{n}$ and $\mathcal{I}_{+}^{a} \cap \mathcal{I}_{+}^{n}=\varnothing$. The weight in (6.36) can then be rewritten as

$$
\begin{aligned}
w_{\mathcal{Y}}^{\left(\mathcal{I}_{+}, \theta\right)} & \propto w_{+}^{\left(\mathcal{I}_{+}\right)} \prod_{\ell \in \mathcal{I}_{+}} \eta_{\mathcal{Y}}^{(\ell, \theta(\ell))} \\
& =\prod_{\ell \in \mathcal{L}_{+}-\mathcal{I}_{+}}\left(1-r_{+}^{(\ell)}\right) \prod_{\ell^{\prime} \in \mathcal{I}_{+}^{a}} r_{+}^{\left(\ell^{\prime}\right)} \eta_{\mathcal{Y}}^{\left(\ell^{\prime}, \theta\left(\ell^{\prime}\right)\right)} \prod_{\ell^{\prime \prime} \in \mathcal{I}_{+}^{n}} r_{+}^{\ell^{\prime \prime}} \eta_{\mathcal{Y}}^{\left(\ell^{\prime \prime}, 0\right)} \\
& =\exp \left(\sum_{\ell \in \mathcal{L}_{+}-\mathcal{I}_{+}} v^{(\ell)}+\sum_{\ell^{\prime} \in \mathcal{I}_{+}^{a}} \alpha^{\left(\ell^{\prime}, \theta\left(\ell^{\prime}\right)\right)}+\sum_{\ell^{\prime \prime} \in \mathcal{I}_{+}^{n}} \mu^{\left(\ell^{\prime \prime}\right)}\right),
\end{aligned}
$$

where each term is obtained as the negative logarithm of the corresponding factor. The three sums represent non-existing targets, associated targets and targets with a missed detection. A valid hypothesis assigns each target to one group and assigns each associated target to a unique measurement.

Each hypothesis $\left(\mathcal{I}_{+}, \theta\right)$ is generated in order of probability using Murty's algorithm $[129,134]$, to create a truncated approximation of the full sums of (6.35). The truncation is achieved through the termination of Murty's algorithm based on either a maximum number of drawn hypotheses or a minimum hypothesis probability and through only considering gated measurements. Clustering of targets and measurements as discussed in Section 6.1.5 is often crucial for good performance. The association matrix for Murty's algorithm is constructed from the terms in (6.38). For the scenario in Figure 6.1 this results in Table 6.3, where the track labels $\mathcal{L}_{+}=\left\{\tau_{1}, \tau_{2}, \tau_{3}\right\}$ are used.

An alternative to using Murty's algorithm, which at best has complexity $\mathcal{O}\left(K(M+2 N)^{3}\right)$, is proposed in [188]. It proposes to use Gibbs sampling, with complexity $\mathcal{O}\left(K N^{2} M\right)$, to obtain a set of high-probability hypotheses. It is seen 
that this scales particularly well in the number of measurements. The motivation for this approach is that adding any hypothesis not already included to the approximation of the sums in (6.35) reduces the approximation error.

\subsubsection{Adaptive Birth Model}

To include new targets in the tracker, the LмB filter relies on a birth distribution. Different birth-models have been discussed in , e.g., [197]. Following [154], the birth model,

$$
\pi_{B}=\left\{\left(r_{B}^{(\ell)}, p_{B}^{(\ell)}\right)\right\}_{\ell \in \mathcal{B}^{\prime}}
$$

is selected such that a new target in $\mathcal{B}$ is generated for each measurement in $\mathcal{Y}$ from the previous time step. The existence probability of a new target $\ell_{i} \in \mathcal{B}$, corresponding to measurement $\mathbf{y}_{i} \in \mathcal{Y}$, is proportional to the probability of the measurement not being associated with any previously known target. The association probability is given by

$$
r_{U}^{i}=\sum_{\mathcal{I}_{+} \in \mathcal{F}\left(\mathcal{L}_{+}\right)} \sum_{\theta \in \Theta_{\mathcal{I}_{+}}} 1_{\theta\left(\mathcal{I}_{+}\right)}(i) w_{\mathcal{Y}}^{\left(\mathcal{I}_{+}, \theta\right)}, \quad i \in\{1:|\mathcal{Y}|\},
$$

where $\theta\left(\mathcal{I}_{+}\right)$is the image of $\theta$. Given an expected number of new targets in each scan, $\lambda_{B}$, the existence probability of the new target is thus given by

$$
r_{B}^{\left(\ell_{i}\right)}=\min \left(r_{B}^{\max }, \frac{\left(1-r_{U}^{i}\right) \cdot \lambda_{B}}{\sum_{i=1}^{|\mathcal{Y}|} 1-r_{U}^{i}}\right) .
$$

The limitation to $r_{B}^{\max } \leq 1$ is needed to ensure that the existence probability $r_{B}^{(i)} \leq 1$ when $\lambda_{B}>1$.

\subsubsection{Reformulation}

The classic LMB filter formulation of [154] carries a heritage from the $\delta$-GLMB implementation, leading to an artificial reconstruction of the $\delta$-GLMB distribution using the $k$-shortest-path algorithm in the measurement update stage. Here we propose a formulation that does not require the $\delta$-GLMB reconstruction due to its immediate collapse into the LMB distribution approximation. This section describes Contribution V.

Note that $\sum_{i=0}^{|\mathcal{Y}|} \delta_{\theta(\ell)}(i)=1$, where $\delta_{a}(b)$ is the Kronecker delta function, defined as 1 if $a=b$ and 0 otherwise. The measurement update parameters in (6.35) can then be reformulated as

$$
\begin{aligned}
r^{(\ell)} & =\sum_{i=0}^{|\mathcal{Y}|}\left[\sum_{\mathcal{I}_{+} \in \mathcal{F}\left(\mathcal{L}_{+}\right)} 1_{\mathcal{I}_{+}}(\ell) \sum_{\theta \in \Theta_{\mathcal{I}_{+}}} w_{\mathcal{Y}}^{\left(\mathcal{I}_{+}, \theta\right)} \cdot \delta_{\theta(\ell)}(i)\right]=\sum_{i=0}^{|\mathcal{Y}|} w^{(\ell, i)}, \\
p^{(\ell)}(\mathbf{x}) & =\frac{1}{r^{(\ell)}} \sum_{i=0}^{|\mathcal{Y}|}\left[\sum_{\mathcal{I}_{+} \in \mathcal{F}\left(\mathcal{L}_{+}\right)} 1_{\mathcal{I}_{+}}(\ell) \sum_{\theta \in \Theta_{\mathcal{I}_{+}}} w_{\mathcal{Y}}^{\left(\mathcal{I}_{+}, \theta\right)} \cdot \delta_{\theta(\ell)}(i) \cdot p^{(\ell, \theta(\ell))}(\mathbf{x} \mid \mathcal{Y})\right]
\end{aligned}
$$




$$
=\frac{1}{r^{(\ell)}} \sum_{i=0}^{|\mathcal{Y}|} w^{(\ell, i)} p^{(\ell, i)}(\mathbf{x} \mid \mathcal{Y}),
$$

where $w^{(\ell, i)}$ denotes the sum of the weights for the hypotheses where target $\ell$ is associated to measurement $\mathbf{y}_{i}$, for $i \in\{1:|\mathcal{Y}|\}$, or missed, for $i=0$. Similarly, by noting that $\sum_{\ell \in \mathcal{L}_{+}} 1_{\mathcal{I}_{+}}(\ell) \delta_{\theta(\ell)}(i)=1_{\theta\left(\mathcal{I}_{+}\right)}(i)$, the association probability in $(6.40)$ can be reformulated as

$$
r_{U}^{i}=\sum_{\ell \in \mathcal{L}_{+}}\left[\sum_{\mathcal{I}_{+} \in \mathcal{F}\left(\mathcal{L}_{+}\right)} 1_{\mathcal{I}_{+}}(\ell) \sum_{\theta \in \Theta_{\mathcal{I}_{+}}} w_{\mathcal{Y}}^{\left(\mathcal{I}_{+}, \theta\right)} \cdot \delta_{\theta(\ell)}(i)\right]=\sum_{\ell \in \mathcal{L}_{+}} w^{(\ell, i)} .
$$

An association probability matrix, see Table 6.4 , is constructed by adding the probability of each hypothesis generated using Murty's algorithm to the appropriate column for each target according to the hypothesis. The matrix is then normalized by the total sum of the probabilities of the generated hypotheses. The row sums give the existence probabilities for the targets and the column sums give the association probabilities for the measurements.

Table 6.4: An example of an association probability matrix for two measurements and three tracks, where each element contains the sum of the weights for hypotheses where the target is associated to the measurement. The rightmost column are for associations to missed detections. Compare with the association probability matrix in Table 6.2 .

\begin{tabular}{cccc}
\hline & \multicolumn{2}{c}{ Associated } & Missed \\
Targets & $\mathbf{y}_{1}$ & $\mathbf{y}_{2}$ & \\
\hline Target $\tau_{1}$ & $w^{\left(\tau_{1}, 1\right)}$ & 0 & $w^{\left(\tau_{1}, 0\right)}$ \\
Target $\tau_{2}$ & $w^{\left(\tau_{2}, 1\right)}$ & $w^{\left(\tau_{2}, 2\right)}$ & $w^{\left(\tau_{2}, 0\right)}$ \\
Target $\tau_{3}$ & 0 & $w^{\left(\tau_{3}, 2\right)}$ & $w^{\left(\tau_{3}, 0\right)}$ \\
\hline
\end{tabular}

\section{Application 6.4: Sea Ice Tracking Results}

Following the procedure outlined in this section combined with spatial indexing for clustering and a PF for estimation, the LMB implementation was used to track the ice movements in Kongsfjorden, Svalbard, Norway, over a period of seven hours, with scans delivered every three minutes. The structure of the target tracking framework is presented in Figure 6.9.

In Figure 6.8, the tracks of ice objects are seen to build up over time. The stationary sea ice, shown in green, changes only slightly over the course of the experiment, suggesting it would remain largely undetected if treated as drift ice. The detections of drift ice, shown in red, suffer many false alarms, but the Lмв filter manages to confirm the targets, shown as ellipses, and maintain their tracks, shown as lines, over large stretches of water. As can be seen in Figure 6.8, each scan consists of hundreds of detections, approximately 150 to 400, and about 50 to 190 targets are being maintained over time by the LMв filter. 


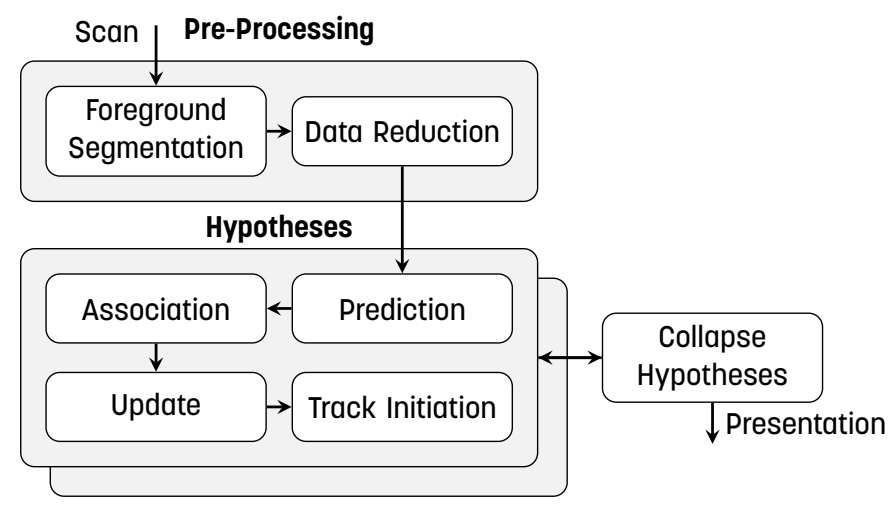

Figure 6.9: The pipeline of processing a scan from the sensor in the sea ice tracking application.

However, the low sampling rate of the TRI allows the filter to run in real-time, despite such conditions.

The sea ice tracking application has demonstrated the spatially indexed LMB filter in practice, showing its viability for tracking sea ice using radar. Future work involves adding more stationary and mobile sensors to the problem formulation to expand the local solution to a global sea ice management framework. This concludes the sea ice tracking application.

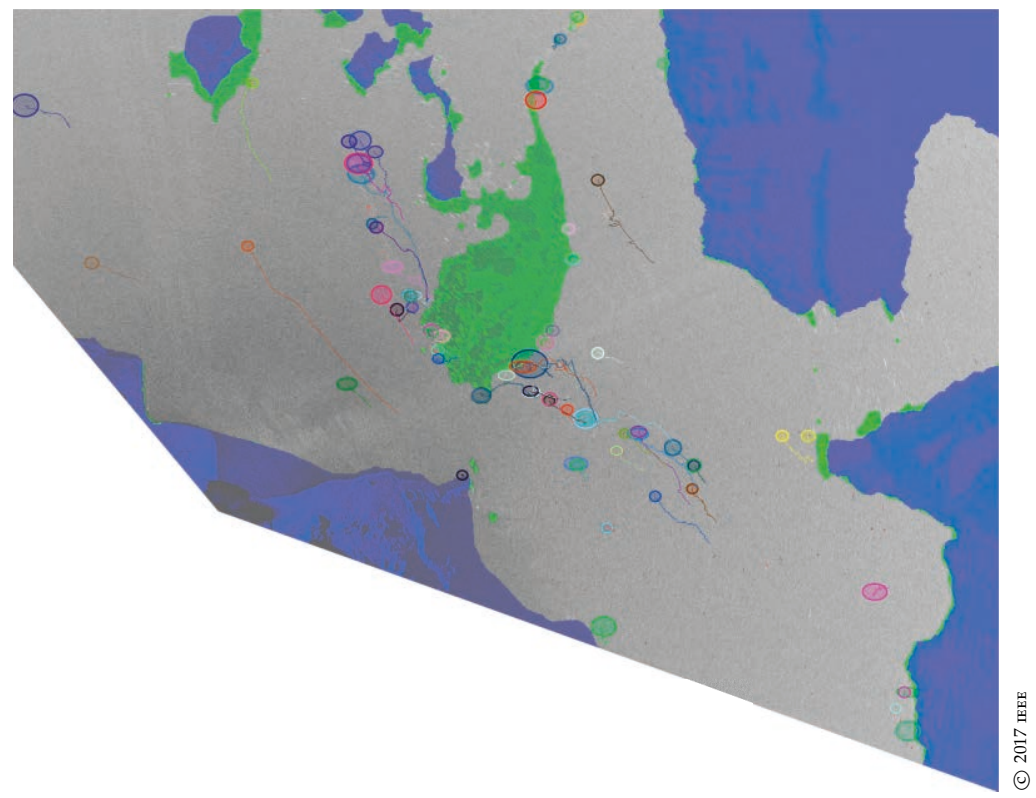

(a) Tracks after $2 h$. 


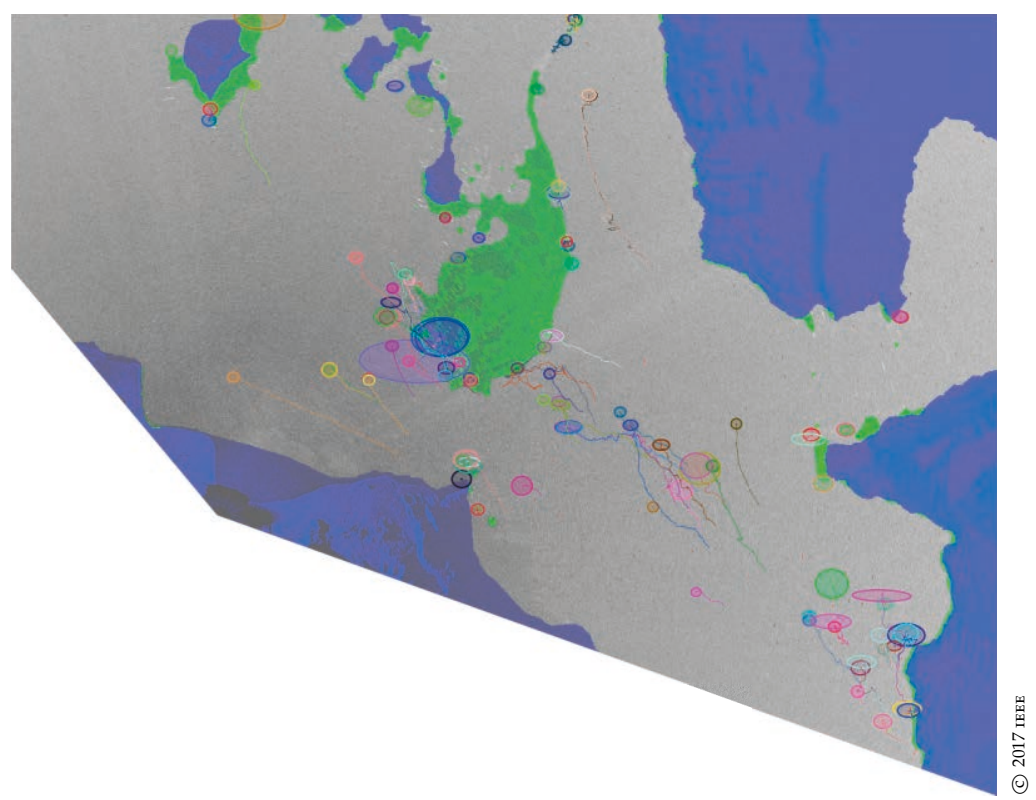

(b) Tracks after $4 h$.

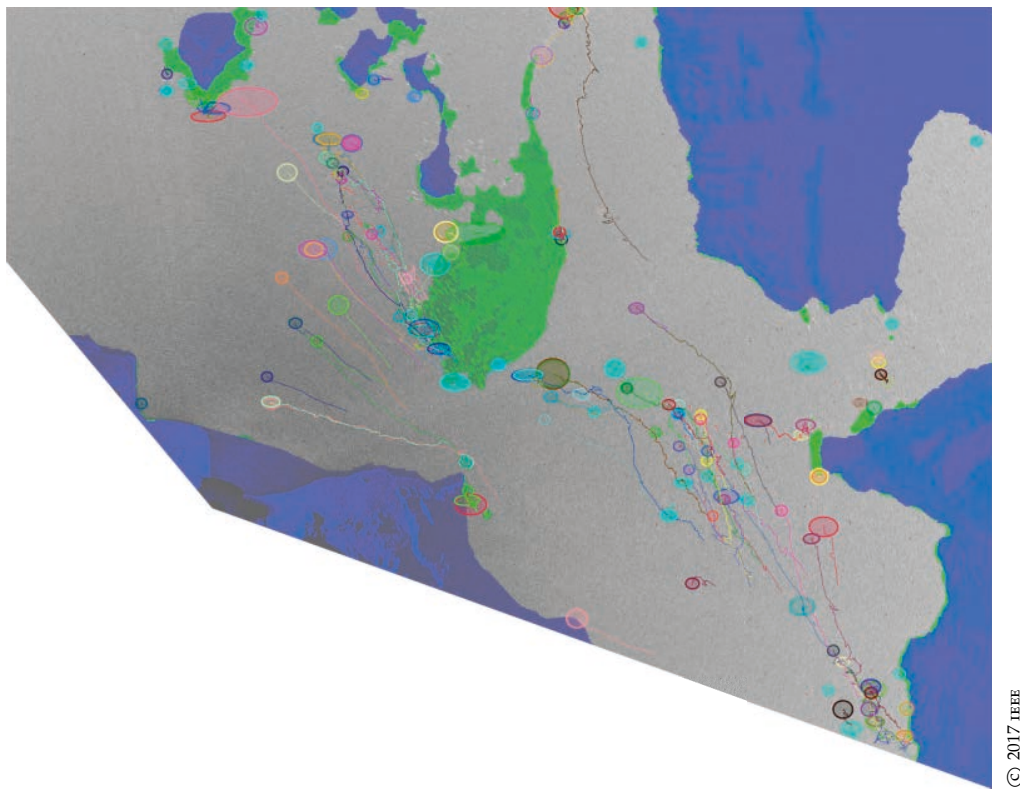

(c) Tracks after $7 \mathrm{~h}$.

Figure 6.8: Drift ice tracks over time, showing the land mask in blue and stationary detections in green. Tracks and targets retain an individual randomly assigned colour over time. Published in [141]. 



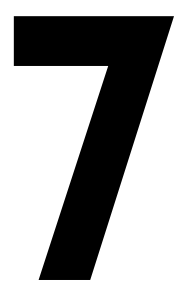

\section{Conclusions and Future Work}

Target tracking has traditionally been a military and aviation research field that has lately diversified into civilian applications due to the development of cheap hardware. Interesting challenges and opportunities arise with new applications, of which a number have been addressed in this thesis. A few established methods for target tracking have been presented with various improvements proposed by the author. A number of applications with experimental and simulated data have been used to verify, demonstrate and illustrate the applicability of the developed theories and methods.

Contribution I was a Gaussian process motion model (GPMM) for learning an influence on the behaviour of targets, such as wind or obstacles, while tracking. A filter for the novel model was derived and several implementation considerations were discussed. To verify and demonstrate the developed theory the model was applied to both a simulated scenario and a sea ice tracking application. Results showed increased accuracy compared to conventional models.

Contribution II was an uncertain timestamp model (UTM) for multiple observations of uncertain time in a linear Gaussian state-space model (LGSSM). The posterior smoothing distributions as well as various common point estimators for the states and the uncertain timestamps were derived. The implications of the additional observations were analysed for simulated scenarios. To further verify and demonstrate the developed theory the model was applied in an orienteering application. Results showed increased accuracy compared to disregarding the observations.

Contribution III was a constrained motion model (CMM) for targets that are confined to a certain space. The novel model results in feasible predictions of a target known to be inside a constraint region, which is impossible for conventional motion models. The model was applied in a dolphin tracking application using a camera where it was shown to improve robustness against occlusions. 
Contribution IV was an extension to the jump Markov model (JMM) incorporating state-independent measurements that directly relate to the mode. A filter bank was developed to estimate the mode and the states of the model. The method was applied in a bird tracking application, where additional measurements, like blurriness, were used to improve the estimation of the bird being in stationary or flight mode. For each take-off, represented by a transition from stationary to flight mode, the direction was extracted. The method was shown to compare well with a manual method for estimating take-off directions. It is also faster, cheaper and more informative than the current state-of-the-art method.

Contribution V was a reformulation of the labelled multi-Bernoulli (LMB) filter that exposes structure that was exploited to construct a more efficient algorithm. The algorithm simultaneously produces both the update and the birth weights for the Lмв distribution while avoiding the explicit construction of intermediate hypotheses, reducing the number of required computations. Sound approximations and clever implementations are essential to achieve good performance for modern target tracking algorithms. The filter was integrated in a full framework for tracking sea ice.

These contributions aim to analyse and propose solutions for several relatively uncharted scenarios in target tracking. The scenarios are relevant for the collaborators and have resulted in several steps towards solutions to the problems they experience. However, several open research questions remain and the strongest candidate for continued work is found in further development of the GPMM. In particular, the scalability of the method must be improved to expand its applicability to other scenarios. The model also needs to be expanded to handle data association in a tractable manner. Further it would be interesting to investigate an extension to the model that would allow learning multi-modal behaviours. It would also be interesting to apply the method to the dolphin application to learn the motion model and compare to the CMM. For the UTM, a filter solution should be investigated to allow an online use observations with uncertain timestamps. 


\section{A}

Proofs

This appendix contains four proofs that have been omitted in the main body of the thesis to improve readability.

\section{A.1 Integrated Weights}

This appendix presents the proof of Proposition 3.1.

Proof: First, decomposing a with respect to the set of unit-length perpendicular basis vectors $\mathbf{b}$ and $\mathbf{b}_{\perp}$ gives

$$
\mathbf{a}=\left(\mathbf{a}^{\top} \mathbf{b}\right) \mathbf{b}+\left(\mathbf{a}^{\top} \mathbf{b}_{\perp}\right) \mathbf{b}_{\perp},
$$

which leads to the relationship

$$
\|\mathbf{a}\|^{2}=\mathbf{a}^{\top} \mathbf{a}=\left(\mathbf{a}^{\top} \mathbf{b}\right)^{2}+\left(\mathbf{a}^{\top} \mathbf{b}_{\perp}\right)^{2} .
$$

Using this relationship, $\mathbf{b}^{\top} \mathbf{b}=1$ and results from [149], the integral is computed analytically by

$$
\begin{aligned}
\int_{0}^{c} \frac{1}{\|\mathbf{a}-s \mathbf{b}\|^{2}} d s & =\int_{0}^{c} \frac{d s}{\mathbf{a}^{\top} \mathbf{a}-2 s \mathbf{a}^{\top} \mathbf{b}+s^{2}}=\int_{0}^{c} \frac{d s}{\left(s-\mathbf{a}^{\top} \mathbf{b}\right)^{2}+\mathbf{a}^{\top} \mathbf{a}-\left(\mathbf{a}^{\top} \mathbf{b}\right)^{2}} \\
& =\int_{0}^{c} \frac{d s}{\left(s-\mathbf{a}^{\top} \mathbf{b}\right)^{2}+\left(\mathbf{a}^{\top} \mathbf{b}_{\perp}\right)^{2}}=\int_{-\mathbf{a}^{\top} \mathbf{b}}^{c-\mathbf{a}^{\top} \mathbf{b}} \frac{d s}{s^{2}+\left(\mathbf{a}^{\top} \mathbf{b}_{\perp}\right)^{2}} \\
& =\frac{1}{\mathbf{a}^{\top} \mathbf{b}_{\perp}}\left(\arctan \left(\frac{c-\mathbf{a}^{\top} \mathbf{b}}{\mathbf{a}^{\top} \mathbf{b}_{\perp}}\right)+\arctan \left(\frac{\mathbf{a}^{\top} \mathbf{b}}{\mathbf{a}^{\top} \mathbf{b}_{\perp}}\right)\right) .
\end{aligned}
$$


The two arctan terms can further be reduced to a single arctan using relationships for sums of arctan functions [149]. Noting that for $\|\mathbf{a}\|^{2} \neq c \mathbf{a}^{\top} \mathbf{b}$

$$
\frac{\frac{c-\mathbf{a}^{\top} \mathbf{b}}{\mathbf{a}^{\top} \mathbf{b}_{\perp}}+\frac{\mathbf{a}^{\top} \mathbf{b}}{\mathbf{a}^{\top} \mathbf{b}_{\perp}}}{1-\frac{c-\mathbf{a}^{\top} \mathbf{b}}{\mathbf{a}^{\top} \mathbf{b}_{\perp}} \cdot \frac{\mathbf{a}^{\top} \mathbf{b}}{\mathbf{a}^{\top} \mathbf{b}_{\perp}}}=\frac{c \mathbf{a}^{\top} \mathbf{b}_{\perp}}{\left(\mathbf{a}^{\top} \mathbf{b}_{\perp}\right)^{2}-c \mathbf{a}^{\top} \mathbf{b}+\left(\mathbf{a}^{\top} \mathbf{b}\right)^{2}}=\frac{c \mathbf{a}^{\top} \mathbf{b}_{\perp}}{\|\mathbf{a}\|^{2}-c \mathbf{a}^{\top} \mathbf{b}^{\prime}},
$$

the reduction is given by

$$
\begin{aligned}
& \int_{0}^{c} \frac{1}{\|\mathbf{a}-s \mathbf{b}\|^{2}} d s \\
& \quad=\frac{1}{\mathbf{a}^{\top} \mathbf{b}_{\perp}} \begin{cases}\pi / 2, & c \mathbf{a}^{\top} \mathbf{b}=\|\mathbf{a}\|^{2}, \mathbf{a}^{\top} \mathbf{b}_{\perp}>0, \\
-\pi / 2, & c \mathbf{a}^{\top} \mathbf{b}=\|\mathbf{a}\|^{2}, \mathbf{a}^{\top} \mathbf{b}_{\perp}<0, \\
\arctan \left(\frac{c \mathbf{a}^{\top} \mathbf{b}_{\perp}}{\|\mathbf{a}\|^{2}-\mathbf{c}^{\top} \mathbf{b}}\right), & c \mathbf{a}^{\top} \mathbf{b}<\|\mathbf{a}\|^{2}, \\
\arctan \left(\frac{c \mathbf{a}_{\perp}^{\top} \mathbf{b}_{\perp}}{\| \mathbf{a}^{2}-c \mathbf{a}^{\top} \mathbf{b}}\right)+\pi, & \mathbf{c}^{\top} \mathbf{b}>\|\mathbf{a}\|^{2}, \mathbf{a}^{\top} \mathbf{b}_{\perp}>0, \\
\arctan \left(\frac{c \mathbf{a}^{\top} \mathbf{b}_{\perp}}{\|\mathbf{a}\|^{2}-c \mathbf{a}^{\top} \mathbf{b}}\right)-\pi, & c \mathbf{a}^{\top} \mathbf{b}>\|\mathbf{a}\|^{2}, \mathbf{a}^{\top} \mathbf{b}_{\perp}<0,\end{cases} \\
& \quad=\frac{1}{\mathbf{a}^{\top} \mathbf{b}_{\perp}} \arctan 2\left(c \mathbf{a}^{\top} \mathbf{b}_{\perp},\|\mathbf{a}\|^{2}-c \mathbf{a}^{\top} \mathbf{b}\right) .
\end{aligned}
$$

The case when $c \mathbf{a}^{\top} \mathbf{b} \geq\|\mathbf{a}\|^{2}$ and $\mathbf{a}^{\top} \mathbf{b}_{\perp}=0$ cannot occur since $\mathbf{a} \neq s \mathbf{b}, \forall s \in$ $[0, c]$.

\section{A.2 State Injection}

This appendix presents the proof of Proposition 5.1.

Proof: The general approach of the proof is as follows. First, relationships between the information matrices and information states of the original and modified models are established. Second, partitioned inversion of the information matrix for the modified model is performed to show the equalities in (5.17).

In the remainder of the proof, the shorthands $\mathbf{F} \triangleq \mathbf{F}_{k, k-1}, \mathbf{F}_{1} \triangleq \mathbf{F}_{\tau, k-1}, \mathbf{F}_{2} \triangleq$ $\mathbf{F}_{k, \tau}, \mathbf{Q} \triangleq \mathbf{Q}_{k, k-1}, \mathbf{Q}_{1} \triangleq \mathbf{Q}_{\tau, k-1}$ and $\mathbf{Q}_{2} \triangleq \mathbf{Q}_{k, \tau}$ are used, as well as the notations

$$
\mathbf{I}_{k, \tau} \mathcal{X}_{m}=\left(\begin{array}{c}
\mathbf{x}_{k-1} \\
\mathbf{x}_{k} \\
\mathbf{x}_{\tau}
\end{array}\right) \text {. }
$$

The information matrix and information state are augmented with an additional state, for which there exists no information, as

$$
\begin{aligned}
\mathcal{I}_{0} & =\left(\begin{array}{ll}
\mathcal{I} & \mathbf{0} \\
\mathbf{0} & \mathbf{0}
\end{array}\right)=\left(\begin{array}{cc}
\mathbf{P}^{-1} & \mathbf{0} \\
\mathbf{0} & \mathbf{0}
\end{array}\right), \\
\iota_{0} & =\left(\begin{array}{l}
\boldsymbol{\iota} \\
\mathbf{0}
\end{array}\right)=\left(\begin{array}{c}
\mathbf{P}^{-1} \hat{\mathcal{X}} \\
\mathbf{0}
\end{array}\right) .
\end{aligned}
$$


Both models contain the same information.The removed equation in (5.15) is rewritten on the form

$$
\begin{aligned}
\mathbf{0} & =\mathbf{H}_{-} \mathcal{X}_{m}+\mathbf{w}_{k, k-1}, \\
\mathbf{H}_{-} & =\left(\begin{array}{lll}
-\mathbf{F} & \mathbf{I} & \mathbf{0}
\end{array}\right) \mathbf{I}_{k, \tau},
\end{aligned}
$$

which is represented on information form by

$$
\begin{aligned}
& \boldsymbol{\iota}_{-}=\mathbf{H}_{-}^{\top} \mathbf{Q}^{-1} \mathbf{0}=\mathbf{0}, \\
& \mathcal{I}_{-}=\mathbf{H}_{-}^{\top} \mathbf{Q}^{-1} \mathbf{H}_{-} .
\end{aligned}
$$

The added equations in (5.16a) and (5.16b) are rewritten on the form

$$
\begin{aligned}
\mathbf{0} & =\mathbf{H}_{1} \mathcal{X}_{m}+\mathbf{w}_{\tau, k-1}, \\
\mathbf{H}_{1} & =\left(\begin{array}{lll}
-\mathbf{F}_{1} & \mathbf{0} & \mathbf{I}
\end{array}\right) \mathbf{I}_{k, \tau},
\end{aligned}
$$

which is represented on information form by

$$
\begin{aligned}
\iota_{1} & =\mathbf{H}_{1}^{\top} \mathbf{Q}_{1}^{-1} \mathbf{0}=\mathbf{0}, \\
\mathcal{I}_{1} & =\mathbf{H}_{1}^{\top} \mathbf{Q}_{1}^{-1} \mathbf{H}_{1},
\end{aligned}
$$

and

$$
\begin{aligned}
\mathbf{0} & =\mathbf{H}_{2} \mathcal{X}_{m}+\mathbf{w}_{k, \tau}, \\
\mathbf{H}_{2} & =\left(\begin{array}{lll}
\mathbf{0} & \mathbf{I} & -\mathbf{F}_{2}
\end{array}\right) \mathbf{I}_{k, \tau},
\end{aligned}
$$

which is represented on information form by

$$
\begin{aligned}
\boldsymbol{\iota}_{2} & =\mathbf{H}_{2}^{\top} \mathbf{Q}_{2}^{-1} \mathbf{0}=\mathbf{0}, \\
\mathcal{I}_{2} & =\mathbf{H}_{2}^{\top} \mathbf{Q}_{2}^{-1} \mathbf{H}_{2} .
\end{aligned}
$$

Thus, the modification in (5.15) and (5.16) applied to (5.11a) and (5.11b) results in the following relationships between the information matrices and states of the original and modified models,

$$
\begin{aligned}
\mathcal{I}_{m} & =\mathcal{I}_{0}+\mathbf{I}_{k, \tau}^{\top}\left(-\mathcal{I}_{-}+\mathcal{I}_{1}+\mathcal{I}_{2}\right) \mathbf{I}_{k, \tau}=\mathcal{I}_{0}+\mathbf{I}_{k, \tau}^{\top} \mathbf{H}_{m}^{\top} \mathbf{R}_{m}^{-1} \mathbf{H}_{m} \mathbf{I}_{k, \tau}, \\
\boldsymbol{\iota}_{m} & =\iota_{0}+\mathbf{I}_{k, \tau}^{\top}\left(-\iota_{-}+\iota_{1}+\iota_{2}\right)=\iota_{0},
\end{aligned}
$$

where

$$
\begin{aligned}
\mathbf{H}_{m} & =\left(\begin{array}{ccc}
-\mathbf{F} & \mathbf{I} & \mathbf{0} \\
-\mathbf{F}_{1} & \mathbf{0} & \mathbf{I} \\
\mathbf{0} & \mathbf{I} & -\mathbf{F}_{2}
\end{array}\right) \\
\mathbf{R}_{m} & =\left(\begin{array}{ccc}
-\mathbf{Q} & \mathbf{0} & \mathbf{0} \\
\mathbf{0} & \mathbf{Q}_{1} & \mathbf{0} \\
\mathbf{0} & \mathbf{0} & \mathbf{Q}_{2}
\end{array}\right)
\end{aligned}
$$


The middle part of the second term in (A.14a) is computed directly as

$$
\begin{aligned}
\mathbf{H}_{m}^{\top} \mathbf{R}_{m}^{-1} \mathbf{H}_{m} & =\left(\begin{array}{cc}
\mathbf{A} & -\mathbf{C}^{\top} \\
-\mathbf{C} & \mathbf{D}
\end{array}\right) \\
& =\left(\begin{array}{ccc}
-\mathbf{F}^{\top} \mathbf{Q}^{-1} \mathbf{F}+\mathbf{F}_{1}^{\top} \mathbf{Q}_{1}^{-1} \mathbf{F}_{1} & \mathbf{F}^{\top} \mathbf{Q}^{-1} & -\mathbf{F}_{1}^{\top} \mathbf{Q}_{1}^{-1} \\
\mathbf{Q}^{-1} \mathbf{F} & -\mathbf{Q}^{-1}+\mathbf{Q}_{2}^{-1} & -\mathbf{Q}_{2}^{-1} \mathbf{F}_{2} \\
-\mathbf{Q}_{1}^{-1} \mathbf{F}_{1} & -\mathbf{F}_{2}^{\top} \mathbf{Q}_{2}^{-1} & \mathbf{Q}_{1}^{-1}+\mathbf{F}_{2}^{\top} \mathbf{Q}_{2}^{-1} \mathbf{F}_{2}
\end{array}\right),
\end{aligned}
$$

where $\mathbf{C}$ and $\mathbf{D}$ correspond to (5.18c) and (5.18b). The resulting information matrix for the modified model in (A.14a) is then given by

$$
\begin{aligned}
\mathcal{I}_{m} & =\mathcal{I}_{0}+\mathbf{I}_{k, \tau}^{\top} \mathbf{H}_{m}^{\top} \mathbf{R}_{m}^{-1} \mathbf{H}_{m} \mathbf{I}_{k, \tau} \\
& =\left(\begin{array}{cc}
\mathcal{I}+\mathbf{I}_{k}^{\top} \mathbf{A I}_{k} & -\mathbf{I}_{k}^{\top} \mathbf{C}^{\top} \\
-\mathbf{C I}_{k} & \mathbf{D}
\end{array}\right)=\left(\begin{array}{cc}
\tilde{\mathbf{A}} & -\tilde{\mathbf{C}}^{\top} \\
-\tilde{\mathbf{C}} & \tilde{\mathbf{D}}
\end{array}\right) .
\end{aligned}
$$

The covariance matrix in (5.17) is obtained using partitioned inversion [91] as

$$
\left[\begin{array}{cc}
\mathbf{P}_{m} & \mathbf{P}_{\tau m}^{\top} \\
\mathbf{P}_{\tau m} & \mathbf{P}_{\tau}
\end{array}\right]=\mathcal{I}_{m}^{-1}=\left(\begin{array}{cc}
\mathbf{E}^{-1} & \mathbf{E}^{-1} \tilde{\mathbf{C}}^{\top} \tilde{\mathbf{D}}^{-1} \\
\tilde{\mathbf{D}}^{-1} \tilde{\mathbf{C}} \mathbf{E}^{-1} & \tilde{\mathbf{D}}^{-1}+\tilde{\mathbf{D}}^{-1} \tilde{\mathbf{C}} \mathbf{E}^{-1} \tilde{\mathbf{C}}^{\top} \tilde{\mathbf{D}}^{-1}
\end{array}\right)
$$

where

$$
\begin{aligned}
\mathbf{E} & =\tilde{\mathbf{A}}-\tilde{\mathbf{C}}^{\top} \tilde{\mathbf{D}}^{-1} \tilde{\mathbf{C}}=\mathcal{I}+\mathbf{I}_{k}^{\top} \mathbf{A} \mathbf{I}_{k}-\mathbf{I}_{k}^{\top} \mathbf{C}^{\top} \mathbf{D}^{-1} \mathbf{C} \mathbf{I}_{k} \\
& =\mathcal{I}+\mathbf{I}_{k}^{\top}\left(\mathbf{A}-\mathbf{C}^{\top} \mathbf{D}^{-1} \mathbf{C}\right) \mathbf{I}_{k} .
\end{aligned}
$$

The next step is to show that $\mathbf{A}=\mathbf{C}^{\top} \mathbf{D}^{-1} \mathbf{C}$, which is achieved by showing that each of the four submatrices in $\mathbf{C}^{\top} \mathbf{D}^{-1} \mathbf{C}$ are equivalent to the corresponding submatrices in A when the assumptions in (3.15) hold. The inverse of $\mathbf{D}$ is computed using the Woodbury formula [91] and assumption (3.15b) as

$$
\begin{aligned}
\mathbf{D}^{-1} & =\left(\mathbf{Q}_{1}^{-1}+\mathbf{F}_{2}^{\top} \mathbf{Q}_{2}^{-1} \mathbf{F}_{2}\right)^{-1} \\
& =\mathbf{Q}_{1}-\mathbf{Q}_{1} \mathbf{F}_{2}^{\top}\left(\mathbf{Q}_{2}+\mathbf{F}_{2} \mathbf{Q}_{1} \mathbf{F}_{2}^{\top}\right)^{-1} \mathbf{F}_{2} \mathbf{Q}_{1} \\
& =\mathbf{Q}_{1}-\mathbf{Q}_{1} \mathbf{F}_{2}^{\top} \mathbf{Q}^{-1} \mathbf{F}_{2} \mathbf{Q}_{1} .
\end{aligned}
$$

Using assumption (3.15a) the top left submatrix of $\mathbf{C}^{\top} \mathbf{D}^{-1} \mathbf{C}$ is given by

$$
\begin{aligned}
\left(-\mathbf{F}_{1}^{\top} \mathbf{Q}_{1}^{-1}\right) \mathbf{D}^{-1}\left(-\mathbf{Q}_{1}^{-1} \mathbf{F}_{1}\right) & =\mathbf{F}_{1}^{\top} \mathbf{Q}_{1}^{-1}\left(\mathbf{Q}_{1}-\mathbf{Q}_{1} \mathbf{F}_{2}^{\top} \mathbf{Q}^{-1} \mathbf{F}_{2} \mathbf{Q}_{1}\right) \mathbf{Q}_{1}^{-1} \mathbf{F}_{1} \\
& =\mathbf{F}_{1}^{\top} \mathbf{Q}_{1}^{-1} \mathbf{F}_{1}-\mathbf{F}_{1}^{\top} \mathbf{F}_{2}^{\top} \mathbf{Q}^{-1} \mathbf{F}_{2} \mathbf{F}_{1} \\
& =\mathbf{F}_{1}^{\top} \mathbf{Q}_{1}^{-1} \mathbf{F}_{1}-\mathbf{F}^{\top} \mathbf{Q}^{-1} \mathbf{F} .
\end{aligned}
$$

The bottom left and transpose of top right submatrices are computed using assumption (3.15) as

$$
\begin{gathered}
\left(-\mathbf{Q}_{2}^{-1} \mathbf{F}_{2}\right) \mathbf{D}^{-1}\left(-\mathbf{Q}_{1}^{-1} \mathbf{F}_{1}\right)=\mathbf{Q}_{2}^{-1} \mathbf{F}_{2}\left(\mathbf{Q}_{1}-\mathbf{Q}_{1} \mathbf{F}_{2}^{\top} \mathbf{Q}^{-1} \mathbf{F}_{2} \mathbf{Q}_{1}\right) \mathbf{Q}_{1}^{-1} \mathbf{F}_{1} \\
=\mathbf{Q}_{2}^{-1}\left(\mathbf{I}-\mathbf{F}_{2} \mathbf{Q}_{1} \mathbf{F}_{2}^{\top} \mathbf{Q}^{-1}\right) \mathbf{F}
\end{gathered}
$$




$$
=\mathbf{Q}_{2}^{-1}\left(\mathbf{Q}_{2}+\mathbf{F}_{2} \mathbf{Q}_{1} \mathbf{F}_{2}^{\top}-\mathbf{F}_{2} \mathbf{Q}_{1} \mathbf{F}_{2}^{\top}\right) \mathbf{Q}^{-1} \mathbf{F}=\mathbf{Q}^{-1} \mathbf{F} .
$$

The bottom right submatrix is computed, using the Woodbury formula backwards and assumption (3.15b), as

$$
\begin{aligned}
\mathbf{Q}_{2}^{-1} \mathbf{F}_{2} \mathbf{D}^{-1} \mathbf{Q}_{2}^{-1} \mathbf{F}_{2} & =\mathbf{Q}_{2}^{-1} \mathbf{F}_{2}\left(\mathbf{Q}_{1}^{-1}+\mathbf{F}_{2}^{\top} \mathbf{Q}_{2}^{-1} \mathbf{F}_{2}\right)^{-1} \mathbf{Q}_{2}^{-1} \mathbf{F}_{2} \\
& =\mathbf{Q}_{2}^{-1} \mathbf{F}_{2}\left(\mathbf{Q}_{1}^{-1}+\mathbf{F}_{2}^{\top} \mathbf{Q}_{2}^{-1} \mathbf{F}_{2}\right)^{-1} \mathbf{Q}_{2}^{-1} \mathbf{F}_{2}+\mathbf{Q}_{2}^{-1}-\mathbf{Q}_{2}^{-1} \\
& =\mathbf{Q}_{2}^{-1}-\left(\mathbf{Q}_{2}+\mathbf{F}_{2}^{\top} \mathbf{Q}_{1} \mathbf{F}_{2}\right)^{-1}=\mathbf{Q}_{2}^{-1}-\mathbf{Q}^{-1}
\end{aligned}
$$

Combining the above equations shows the equality

$$
\mathbf{C}^{\top} \mathbf{D}^{-1} \mathbf{C}=\left(\begin{array}{cc}
-\mathbf{F}^{\top} \mathbf{Q}^{-1} \mathbf{F}+\mathbf{F}_{1}^{\top} \mathbf{Q}_{1}^{-1} \mathbf{F}_{1} & \mathbf{F}^{\top} \mathbf{Q}^{-1} \\
\mathbf{Q}^{-1} \mathbf{F} & -\mathbf{Q}^{-1}+\mathbf{Q}_{2}^{-1}
\end{array}\right)=\mathbf{A},
$$

which reduces (A.19) to

$$
\mathbf{E}=\mathcal{I}=\mathbf{P}^{-1}=\mathbf{P}_{m}^{-1}
$$

The cross-covariance $\mathbf{P}_{\tau m}$ in (A.18) reduces to

$$
\mathbf{P}_{\tau m}=\tilde{\mathbf{D}}^{-1} \tilde{\mathbf{C}} \mathbf{E}^{-1}=\mathbf{D}^{-1} \mathbf{C} \mathbf{I}_{k} \mathbf{P}=\mathbf{K} \mathbf{P},
$$

and the covariance $\mathbf{P}_{\tau}$ in (A.18) reduces to

$$
\begin{aligned}
\mathbf{P}_{\tau} & =\tilde{\mathbf{D}}^{-1}+\tilde{\mathbf{D}}^{-1} \tilde{\mathbf{C}} \mathbf{E}^{-1} \tilde{\mathbf{C}}^{\top} \tilde{\mathbf{D}}^{-1} \\
& =\mathbf{D}^{-1}+\mathbf{D}^{-1} \mathbf{C} \mathbf{I}_{k} \mathbf{E}^{-1} \mathbf{I}_{k}^{\top} \mathbf{C}^{\top} \mathbf{D}^{-1} \\
& =\mathbf{D}^{-1}+\mathbf{K} \mathbf{P} \mathbf{K}^{\top},
\end{aligned}
$$

using (A.17), (A.25) and (5.18d). This proves the covariance relation in (5.17). The expectation in (5.17) reduces to

$$
\begin{aligned}
{\left[\begin{array}{c}
\hat{\mathcal{X}}_{m} \\
\hat{\mathbf{x}}_{\tau}
\end{array}\right] } & =\mathcal{I}_{m}^{-1} \iota_{m}=\mathcal{I}_{m}^{-1} \iota_{0}=\left(\begin{array}{c}
\mathbf{E}^{-1} \boldsymbol{\iota} \\
\tilde{\mathbf{D}}^{-1} \tilde{\mathbf{C}} \mathbf{E}^{-1} \boldsymbol{\iota}
\end{array}\right) \\
& =\left(\begin{array}{c}
\mathcal{I}^{-1} \boldsymbol{\iota} \\
\mathbf{D}^{-1} \mathbf{C I}_{k} \mathcal{I}^{-1} \iota
\end{array}\right)=\left(\begin{array}{c}
\hat{\mathcal{X}} \\
\mathbf{K} \hat{\mathcal{X}}
\end{array}\right),
\end{aligned}
$$

using (5.10), (A.14b), (A.7b), (A.18), (A.25) and (A.26). This proves the expectation relation in (5.17) and concludes the proof.

Remark A.1: To avoid inversion of $\mathbf{Q}_{1}$ and $\mathbf{Q}_{2}$, which might cause numerical instability for $t_{\tau}$ near $t_{k}$ or $t_{k-1}$, let $\mathbf{G}=\mathbf{Q}_{1} \mathbf{F}_{2}^{\top} \mathbf{Q}^{-1}$ and rewrite

$$
\begin{aligned}
\mathbf{D}^{-1} & =\mathbf{Q}_{1}-\mathbf{Q}_{1} \mathbf{F}_{2}^{\top} \mathbf{Q}^{-1} \mathbf{F}_{2} \mathbf{Q}_{1}=\left(\mathbf{I}-\mathbf{G F}_{2}\right) \mathbf{Q}_{1}, \\
\mathbf{K} & =\mathbf{D}^{-1} \mathbf{C}=\left(\begin{array}{ll}
\left(\mathbf{I}-\mathbf{G F}_{2}\right) \mathbf{F}_{1} & \mathbf{G}
\end{array}\right),
\end{aligned}
$$

where $\mathbf{D}^{-1}$ and the left submatrix of $\mathbf{K}$ follow directly. The right submatrix assumes that $\mathbf{F}_{2}$ is invertible and is derived as

$$
\mathbf{D}^{-1} \mathbf{F}_{2}^{\top} \mathbf{Q}_{2}^{-1}=\left(\mathbf{Q}_{1}^{-1}+\mathbf{F}_{2}^{\top} \mathbf{Q}_{2}^{-1} \mathbf{F}_{2}\right)^{-1}\left(\left(\mathbf{F}_{2}^{\top} \mathbf{Q}_{2}^{-1}\right)^{-1}\right)^{-1}
$$




$$
\begin{aligned}
& =\left(\left(\mathbf{F}_{2}^{\top} \mathbf{Q}_{2}^{-1}\right)^{-1}\left(\mathbf{Q}_{1}^{-1}+\mathbf{F}_{2}^{\top} \mathbf{Q}_{2}^{-1} \mathbf{F}_{2}\right)\right)^{-1}=\left(\mathbf{Q}_{2} \mathbf{F}_{2}^{-\top}\left(\mathbf{Q}_{1}^{-1}+\mathbf{F}_{2}^{\top} \mathbf{Q}_{2}^{-1} \mathbf{F}_{2}\right)\right)^{-1} \\
& =\left(\mathbf{Q}_{2} \mathbf{F}_{2}^{-\top} \mathbf{Q}_{1}^{-1}+\mathbf{F}_{2}\right)^{-1}=\left(\left(\mathbf{Q}_{2}+\mathbf{F}_{2} \mathbf{Q}_{1} \mathbf{F}_{2}^{\top}\right) \mathbf{F}_{2}^{-\top} \mathbf{Q}_{1}^{-1}\right)^{-1} \\
& =\mathbf{Q}_{1} \mathbf{F}_{2}^{\top}\left(\mathbf{Q}_{2}+\mathbf{F}_{2} \mathbf{Q}_{1} \mathbf{F}_{2}^{\top}\right)^{-1}=\mathbf{Q}_{1} \mathbf{F}_{2}^{\top} \mathbf{Q}^{-1}=\mathbf{G} .
\end{aligned}
$$

\section{A.3 Smoother Update}

This section presents the proof for Proposition 5.2. The derivation is also available as supplementary material for the publication in [183].

Proof: The joint distribution of the states and the new observation is given by [81]

$$
\begin{aligned}
& p(\mathcal{X}, \mathbf{z} \mid \mathcal{Y})=\mathcal{N}\left(\left[\begin{array}{c}
\mathcal{X} \\
\mathbf{z}
\end{array}\right] \mid\left[\begin{array}{c}
\hat{\mathcal{X}} \\
\overline{\mathbf{H}}_{\tau} \hat{\mathcal{X}}
\end{array}\right],\left[\begin{array}{cc}
\mathbf{P} & \mathbf{P} \overline{\mathbf{H}}_{\tau}^{\top} \\
\overline{\mathbf{H}}_{\tau} \mathbf{P} & \overline{\mathbf{H}}_{\tau} \mathbf{P} \overline{\mathbf{H}}_{\tau}^{\top}+\mathbf{R}
\end{array}\right]\right) \\
& =\mathcal{N}\left(\left[\begin{array}{c}
\mathcal{X} \\
\mathbf{z}
\end{array}\right] \mid\left[\begin{array}{c}
\hat{\mathcal{X}} \\
\mathbf{H} \hat{\mathbf{x}}_{\tau}
\end{array}\right],\left[\begin{array}{cc}
\mathbf{P} & \mathbf{P}_{\cdot \tau} \mathbf{H}^{\top} \\
\mathbf{H} \mathbf{P}_{\tau} & \mathbf{H} \mathbf{P}_{\tau} \mathbf{H}^{\top}+\mathbf{R}
\end{array}\right]\right)
\end{aligned}
$$

where $\overline{\mathbf{H}}_{\tau}$ is a sparse matrix defined such that $\overline{\mathbf{H}}_{\tau} \mathcal{X}=\mathbf{H} \mathbf{x}_{\tau}$. The covariance notation used is $\mathbf{P}_{i, j}=\operatorname{Cov}\left(\mathbf{x}_{i}, \mathbf{x}_{j}\right)$ with the shorthand $\mathbf{P}_{i}=\mathbf{P}_{i, i}$ and $\mathbf{P}_{. i}=\operatorname{Cov}\left(\mathcal{X}, \mathbf{x}_{i}\right)$. Also, let $\mathbf{S}_{\tau}=\mathbf{H} \mathbf{P}_{\tau} \mathbf{H}^{\top}+\mathbf{R}$ for simpler notation. Given an observation $\mathbf{z}$ at time $t_{\tau}$ the conditional distribution of $\mathcal{X}$ is given by [81]

$$
\begin{aligned}
p(\mathcal{X} \mid \mathcal{Y}, \mathbf{z}) & =\mathcal{N}\left(\mathcal{X} \mid \hat{\mathcal{X}}+\mathbf{P}_{\cdot \tau} \mathbf{H}^{\top} \mathbf{S}_{\tau}^{-1}\left(\mathbf{z}-\mathbf{H} \hat{\mathbf{x}}_{\tau}\right), \mathbf{P}-\mathbf{P}_{{ }_{\tau}} \mathbf{H}^{\top} \mathbf{S}_{\tau}^{-1} \mathbf{H} \mathbf{P}_{\tau \cdot}\right) \\
& =\mathcal{N}\left(\mathcal{X} \mid \hat{\mathcal{X}}^{+}, \mathbf{P}^{+}\right),
\end{aligned}
$$

where the superscript + denotes the conditional quantity. The full conditional distribution in (A.32) can be broken down into marginal distributions and crosscovariances for individual states as

$$
\begin{aligned}
\hat{\mathbf{x}}_{i}^{+} & =\hat{\mathbf{x}}_{i}+\mathbf{P}_{i, \tau} \mathbf{H}^{\top} \mathbf{S}_{\tau}^{-1}\left(\mathbf{z}-\mathbf{H} \hat{\mathbf{x}}_{\tau}\right), \\
\mathbf{P}_{i, j}^{+} & =\mathbf{P}_{i, j}-\mathbf{P}_{i, \tau} \mathbf{H}^{\top} \mathbf{S}_{\tau}^{-1} \mathbf{H} \mathbf{P}_{\tau, j} .
\end{aligned}
$$

Letting $i=j=\tau$ in (A.33) directly gives (5.21).

The covariance matrix $\mathbf{P}$ is assumed to have a structure that satisfies

$$
\mathbf{P}_{i, j}=\mathbf{P}_{i, i+1} \mathbf{P}_{i+1}^{-1} \mathbf{P}_{i+1, j}, \quad i<j .
$$

This assumption will be shown by induction to hold for the LGSSM, but can be shown to be a general result for inverses of symmetric block tridiagonal matrices using results in [127] under satisfied conditions. Through expansion of the assumption it follows that

$$
\mathbf{P}_{i, j}=\mathbf{P}_{i, i+1} \prod_{k=i+1}^{j-1} \mathbf{P}_{k}^{-1} \mathbf{P}_{k, k+1}=\mathbf{P}_{i, j-1} \mathbf{P}_{j-1}^{-1} \mathbf{P}_{j-1, j}
$$


and using the symmetry of the covariance matrix, $\mathbf{P}_{i, j}^{\top}=\mathbf{P}_{j, i}$, similar results for $i>j$ are obtained by transposing (A.34) and (A.35).

The implication of the assumption (A.34) is that, given the covariance of each state and the cross-covariance of consecutive states, the cross-covariance between any states can be determined. The conditional distribution for the states $\mathbf{x}_{k}$ and the cross-covariances $\mathbf{P}_{k, k+1}$ between consecutive states for $k<\tau$ are derived from (A.33) as

$$
\begin{aligned}
\hat{\mathbf{x}}_{k}^{+} & =\hat{\mathbf{x}}_{k}+\mathbf{P}_{k, \tau} \mathbf{H}^{\top} \mathbf{S}^{-1}\left(\mathbf{z}-\mathbf{H} \hat{\mathbf{x}}_{\tau}\right) \\
& =\hat{\mathbf{x}}_{k}+\mathbf{P}_{k, k+1} \mathbf{P}_{k+1}^{-1} \mathbf{P}_{k+1, \tau} \mathbf{H}^{\top} \mathbf{S}^{-1}\left(\mathbf{z}-\mathbf{H} \hat{\mathbf{x}}_{\tau}\right) \\
& =\hat{\mathbf{x}}_{k}-\mathbf{P}_{k, k+1} \mathbf{P}_{k+1}^{-1}\left(\hat{\mathbf{x}}_{k+1}-\hat{\mathbf{x}}_{k+1}^{+}\right), \\
\mathbf{P}_{k, k+1}^{+} & =\mathbf{P}_{k, k+1}-\mathbf{P}_{k, \tau} \mathbf{H}^{\top} \mathbf{S}^{-1} \mathbf{H} \mathbf{P}_{\tau, k+1} \\
& =\mathbf{P}_{k, k+1}-\mathbf{P}_{k, k+1} \mathbf{P}_{k+1}^{-1} \mathbf{P}_{k+1, \tau} \mathbf{H}^{\top} \mathbf{S}^{-1} \mathbf{H} \mathbf{P}_{\tau, k+1} \\
& =\mathbf{P}_{k, k+1}-\mathbf{P}_{k, k+1} \mathbf{P}_{k+1}^{-1}\left(\mathbf{P}_{k+1}-\mathbf{P}_{k+1}^{+}\right) \\
& =\mathbf{P}_{k, k+1} \mathbf{P}_{k+1}^{-1} \mathbf{P}_{k+1}^{+} \\
\mathbf{P}_{k}^{+} & =\mathbf{P}_{k}-\mathbf{P}_{k, \tau} \mathbf{H}^{\top} \mathbf{S}^{-1} \mathbf{H} \mathbf{P}_{\tau, k} \\
& =\mathbf{P}_{k}-\mathbf{P}_{k, k+1} \mathbf{P}_{k+1}^{-1} \mathbf{P}_{k+1, \tau} \mathbf{H}^{\top} \mathbf{S}^{-1} \mathbf{H} \mathbf{P}_{\tau, k+1} \mathbf{P}_{k+1}^{-1} \mathbf{P}_{k+1, k} \\
& =\mathbf{P}_{k}-\left(\mathbf{P}_{k, k+1}-\mathbf{P}_{k, k+1}^{+}\right) \mathbf{P}_{k+1}^{-1} \mathbf{P}_{k, k+1}^{\top}
\end{aligned}
$$

using the assumption (A.34). By denoting $\mathbf{K}_{k}^{b}=\mathbf{P}_{k, k+1} \mathbf{P}_{k+1}^{-1}$, (5.22) is obtained directly from (A.36). Similar derivations for $k>\tau$ results in (5.23), with $\mathbf{K}_{k}^{f}=$ $\mathbf{P}_{k, k-1} \mathbf{P}_{k-1}^{-1}$. Note that the update of the marginal posterior distribution only depends on the cross-covariances between consecutive states and not the full state covariance. As a result, only the state means, state covariances and crosscovariances between consecutive states are needed to compute the marginal posterior distribution.

The conditional cross-covariances in (A.33b) are for $j \leq \tau$ and $i<j$ obtained as

$$
\begin{aligned}
\mathbf{P}_{i, j}^{+} & =\mathbf{P}_{i, j}-\mathbf{P}_{i, \tau} \mathbf{H}^{\top} \mathbf{S}_{\tau}^{-1} \mathbf{H} \mathbf{P}_{\tau, j} \\
& =\mathbf{P}_{i, i+1} \mathbf{P}_{i+1}^{-1} \mathbf{P}_{i+1, j}-\mathbf{P}_{i, i+1} \mathbf{P}_{i+1}^{-1} \mathbf{P}_{i+1, \tau} \mathbf{H}^{\top} \mathbf{S}^{-1} \mathbf{H} \mathbf{P}_{\tau, j} \\
& =\mathbf{P}_{i, i+1} \mathbf{P}_{i+1}^{-1} \mathbf{P}_{i+1, j}+\mathbf{P}_{i, i+1} \mathbf{P}_{i+1}^{-1}\left(\mathbf{P}_{i+1, j}^{+}-\mathbf{P}_{i+1, j}\right) \\
& =\mathbf{P}_{i, i+1} \mathbf{P}_{i+1}^{-1} \mathbf{P}_{i+1, j}^{+}=\mathbf{P}_{i, i+1}^{+}\left(\mathbf{P}_{i+1}^{+}\right)^{-1} \mathbf{P}_{i+1, j}^{+}
\end{aligned}
$$

using (A.36b) in the last equality. For $j>\tau$ and $i<j$ the cross-covariances are obtained as

$$
\begin{aligned}
\mathbf{P}_{i, j}^{+} & =\mathbf{P}_{i, j}-\mathbf{P}_{i, \tau} \mathbf{H}^{\top} \mathbf{S}_{\tau}^{-1} \mathbf{H} \mathbf{P}_{\tau, j} \\
& =\mathbf{P}_{i, j-1} \mathbf{P}_{j-1}^{-1} \mathbf{P}_{j-1, j}-\mathbf{P}_{i, \tau} \mathbf{H}^{\top} \mathbf{S}^{-1} \mathbf{H} \mathbf{P}_{\tau, j-1} \mathbf{P}_{j-1}^{-1} \mathbf{P}_{j-1, j} \\
& =\mathbf{P}_{i, j-1}^{+} \mathbf{P}_{j-1}^{-1} \mathbf{P}_{j-1, j}=\mathbf{P}_{i, j-1}^{+}\left(\mathbf{P}_{j-1}^{+}\right)^{-1} \mathbf{P}_{j-1, j}^{+}
\end{aligned}
$$


using (A.36b) in the last equality. These are equivalent to (A.34) and (A.35), respectively, showing that the assumption (A.34) still holds for the conditional distribution. Considering the discretized LGSSM in (3.13a) with the prior (3.13c) and no measurements, the covariance between states $\mathbf{x}_{i}$ and $\mathbf{x}_{j}$ for $i<j$ is given by

$$
\begin{aligned}
\mathbf{P}_{i, j} & =\operatorname{Cov}\left(\mathbf{x}_{i}, \mathbf{x}_{j}\right)=\mathbf{E}\left(\left(\mathbf{x}_{i}-\hat{\mathbf{x}}_{i}\right)\left(\mathbf{x}_{j}-\hat{\mathbf{x}}_{j}\right)^{\top}\right) \\
& =\mathbf{E}\left(\left(\mathbf{x}_{i}-\hat{\mathbf{x}}_{i}\right)\left(\mathbf{F}_{j}\left(\mathbf{x}_{j-1}-\hat{\mathbf{x}}_{j-1}\right)+\mathbf{v}_{j}\right)^{\top}\right) \\
& =\mathbf{E}\left(\left(\mathbf{x}_{i}-\hat{\mathbf{x}}_{i}\right)\left(\mathbf{x}_{j-1}-\hat{\mathbf{x}}_{j-1}\right)^{\top}\right) \mathbf{F}_{j}^{\top}=\mathbf{P}_{i, j-1} \mathbf{F}_{j}^{\top} \\
& =\mathbf{P}_{i, j-1} \mathbf{P}_{j-1}^{-1} \mathbf{P}_{j-1} \mathbf{F}_{j}^{\top}=\mathbf{P}_{i, j-1} \mathbf{P}_{j-1}^{-1} \mathbf{P}_{j-1, j} .
\end{aligned}
$$

This shows inductively that the assumption (A.34) holds initially before conditioning on measurements, concluding the proof.

\section{A.4 Uniform Ellipse}

This appendix presents the proof of Proposition 5.3.

Proof: The area of the ellipse is given by [84]

$$
A_{\mathcal{C}}=\frac{\pi}{\sqrt{\left|\mathbf{C}^{-1}\right|}}=\pi|\mathbf{A}| \text {. }
$$

The mean of the stochastic variable is computed as

$$
\mathbf{E}(\mathbf{x})=\int \mathbf{x} p(\mathbf{x}) \mathrm{d} \mathbf{x}=\frac{1}{\pi|\mathbf{A}|} \int_{\mathcal{C}} \mathbf{x} \mathrm{d} \mathbf{x} .
$$

Substitution of $\mathbf{x}=\mathbf{A} \tilde{\mathbf{x}}+\mathbf{b}$, with $\mathrm{d} \mathbf{x}=|\mathbf{A}| \mathrm{d} \tilde{\mathbf{x}}$, gives

$$
\mathbf{E}(\mathbf{x})=\frac{1}{\pi|\mathbf{A}|} \int_{\tilde{\mathbf{x}}^{\top} \tilde{\mathbf{x}} \leq 1}(\mathbf{A} \tilde{\mathbf{x}}+\mathbf{b})|\mathbf{A}| \mathrm{d} \tilde{\mathbf{x}}=\frac{1}{\pi} \int_{\tilde{\mathbf{x}}^{\top} \tilde{\mathbf{x}} \leq 1}(\mathbf{A} \tilde{\mathbf{x}}+\mathbf{b}) \mathrm{d} \tilde{\mathbf{x}} .
$$

Substitution to polar coordinates, $\tilde{\mathbf{x}}=(r \cos (\alpha), r \sin (\alpha))^{\top}$ and $\mathrm{d} \tilde{\mathbf{x}}=r \mathrm{~d} r \mathrm{~d} \alpha$, gives

$$
\begin{aligned}
\mathbf{E}(\mathbf{x}) & =\frac{1}{\pi} \int_{0}^{2 \pi} \int_{0}^{1}\left(\mathbf{A}\left(\begin{array}{c}
r \cos (\alpha) \\
r \sin (\alpha)
\end{array}\right)+\mathbf{b}\right) r \mathrm{~d} r \mathrm{~d} \alpha \\
& =\frac{1}{\pi} \mathbf{b} \int_{0}^{2 \pi} \int_{0}^{1} r \mathrm{~d} r \mathrm{~d} \alpha=\frac{1}{\pi} \mathbf{b} \cdot \pi=\mathbf{b} .
\end{aligned}
$$

The covariance of the stochastic variable is computed as

$$
\operatorname{Cov}(\mathbf{x})=\mathbf{E}\left((\mathbf{x}-\mathbf{E}(\mathbf{x}))(\mathbf{x}-\mathbf{E}(\mathbf{x}))^{\top}\right)=\frac{1}{\pi|\mathbf{A}|} \int_{\mathcal{C}}(\mathbf{x}-\mathbf{b})(\mathbf{x}-\mathbf{b})^{\top} \mathrm{d} \mathbf{x} .
$$


Substitution of $\mathbf{x}=\mathbf{A} \tilde{\mathbf{x}}+\mathbf{b}$, with $\mathrm{d} \mathbf{x}=|\mathbf{A}| \mathrm{d} \tilde{\mathbf{x}}$, gives

$$
\operatorname{Cov}(\mathbf{x})=\frac{1}{\pi} \int_{\tilde{\mathbf{x}}^{\top} \tilde{\mathbf{x}} \leq 1} \mathbf{A}\left(\tilde{\mathbf{x}} \tilde{\mathbf{x}}^{\top}\right) \mathbf{A}^{\top} \mathrm{d} \tilde{\mathbf{x}} .
$$

Substitution to polar coordinates, $\tilde{\mathbf{x}}=(r \cos (\alpha), r \sin (\alpha))^{\top}$ and $\mathrm{d} \tilde{\mathbf{x}}=r \mathrm{~d} r \mathrm{~d} \alpha$, gives

$$
\begin{aligned}
\operatorname{Cov}(\mathbf{x}) & =\frac{1}{\pi} \mathbf{A}\left(\int_{0}^{2 \pi} \int_{0}^{1} r^{3}\left(\begin{array}{cc}
\cos ^{2}(\alpha) & \sin (\alpha) \cos (\alpha) \\
\sin (\alpha) \cos (\alpha) & \sin ^{2}(\alpha)
\end{array}\right) \mathrm{d} r \mathrm{~d} \alpha\right) \mathbf{A}^{\top} \\
& =\frac{1}{\pi} \mathbf{A}\left(\frac{1}{4} \cdot \pi \mathbf{I}_{2}\right) \mathbf{A}^{\top}=\frac{\mathbf{A} \mathbf{A}^{\top}}{4}=\frac{\mathbf{C}}{4}
\end{aligned}
$$




\section{Bibliography}

[1] R. A. Adams. Calculus: A Complete Course. Pearson Education Canada, Toronto, Ontario, Canada, fifth edition, 2003. ISBN 978-0-201-79131-0. Cited on page 104.

[2] J. Ajgl, M. Šimandl, and J. Duník. Millmans formula in data fusion. In Proceedings of the International PhD Workshop Young Generation Viewpoint, Hluboka nad Vltavou, Czech Republic, Sept. 2009. Cited on page 113.

[3] H. Akashi and H. Kumamoto. Random sampling approach to state estimation in switching environments. Automatica, 13(4):429-434, July 1977. ISSN 0005-1098. doi:10.1016/0005-1098(77)90028-0. Cited on page 63.

[4] A. G. Akritas, E. K. Akritas, and G. I. Malaschonok. Various proofs of Sylvester's (determinant) identity. Mathematics and Computers in Simulation, 42(4-6):585-593, Nov. 1996. ISSN 0378-4754. doi:10.1016/S0378-4754(96)00035-3. Cited on page 113.

[5] B. D. O. Anderson and J. B. Moore. Optimal Filtering. Dover Publications, Mineola, NY, USA, 1979. ISBN 978-0-486-43938-9. Cited on page 7.

[6] K. J. Åström. Introduction to Stochastic Control Theory. Dover Publications, Mineola, NY, USA, 2006. ISBN 978-0-486-44531-1. Cited on pages 35, 36, and 37.

[7] M. Athans and C.-B. Chang. Adaptive estimation and parameter identification using multiple model estimation algorithm. Technical Report ESDTR-76-184, Massachusetts Institute of Technology, Lexington, MA, USA, 1976. Cited on page 7.

[8] D. J. Ballantyne, H. Y. Chan, and M. A. Kouritzin. Novel branching particle method for tracking. In Proceedings of SPIE's International Symposium on Aerospace and Defense Sensing, Simulations and Controls, volume 4048, pages 277-287, Orlando, FL, USA, Apr. 2000. doi:10.1117/12.391984. Cited on page 5 . 
[9] Y. Bar-Shalom, editor. Multitarget-Multisensor Tracking: Applications and Advances, volume II. Artech House, Norwood, MA, USA, 1992. ISBN 978-0-89006-517-4. Cited on page 133.

[10] Y. Bar-Shalom. Update with out-of-sequence measurements in tracking: exact solution. IEEE Transactions on Aerospace and Electronic Systems, 38(3): 769-777, July 2002. ISSN 0018-9251. doi:10.1109/TAES.2002.1039398. Cited on pages 1 and 24 .

[11] Y. Bar-Shalom and T. Fortmann. Tracking and Data Association. Academic Press, San Diego, CA, USA, 1988. ISBN 978-0-12-079760-8. Cited on pages $5,6,51,133$, and 143 .

[12] Y. Bar-Shalom and X. R. Li. Multitarget-multisensor Tracking: Principles and Techniques. YBS Publishing, Storrs, CT, USA, 1995. ISBN 978-0-9648312-0-9. Cited on pages 5 and 133.

[13] Y. Bar-Shalom and G. D. Marcus. Tracking with measurements of uncertain origin and random arrival times. IEEE Transactions on Automatic Control, 25(4):802-807, Aug. 1980. ISSN 0018-9286. doi:10.1109/TAC.1980.1102425. Cited on page 24 .

[14] Y. Bar-Shalom and E. Tse. Tracking in a cluttered environment with probabilistic data association. Automatica, 11(5):451-460, Sept. 1975. ISSN 0005-1098. doi:10.1016/0005-1098(75)90021-7. Cited on page 143.

[15] Y. Bar-Shalom, T. Kirubarajan, and R. X. Li. Estimation with Applications to Tracking and Navigation. John Wiley \& Sons, New York, NY, USA, 2002. ISBN 0471221279. Cited on page 38.

[16] Y. Bar-Shalom, H. Chen, and M. Mallick. One-step solution for the multistep out-of-sequence-measurement problem in tracking. IEEE Transactions on Aerospace and Electronic Systems, 40(1):27-37, Jan. 2004. ISSN 0018-9251. doi:10.1109/TAES.2004.1292140. Cited on pages 1 and 24.

[17] Y. Bar-Shalom, X. Li, and T. Kirubarajan. Estimation with Applications to Tracking and Navigation: Theory Algorithms and Software. Wiley, New York, NY, USA, 2004. ISBN 978-0-471-46521-8. Cited on pages 5 and 133.

[18] Y. Bar-Shalom, S. S. Blackman, and R. J. Fitzgerald. Dimensionless score function for multiple hypothesis tracking. IEEE Transactions on Aerospace and Electronic Systems, 43(1):392-400, Jan. 2007. ISSN 0018-9251. doi:10.1109/TAES.2007.357141. Cited on pages 135 and 136.

[19] L. E. Baum, T. Petrie, G. Soules, and N. Weiss. A maximization technique occurring in the statistical analysis of probabilistic functions of Markov chains. The Annals of Mathematical Statistics, 41(1):164-171, Feb. 1970. doi:10.1214/aoms/1177697196. Cited on page 93. 
[20] M. Baum and U. D. Hanebeck. Random hypersurface models for extended object tracking. In Proceedings of the IEEE International Symposium on Signal Processing and Information Technology, pages 178-183, Ajman, UAE, Dec. 2009. doi:10.1109/ISSPIT.2009.5407526. Cited on page 52.

[21] B. M. Bell. The iterated Kalman smoother as a Gauss-Newton method. SIAM Journal on Optimization, 4(3):626-636, 1994. doi:10.1137/0804035. Cited on page 88.

[22] B. M. Bell and F. W. Cathey. The iterated Kalman filter update as a GaussNewton method. IEEE Transactions on Automatic Control, 38(2):294-297, Feb. 1993. ISSN 0018-9286. doi:10.1109/9.250476. Cited on page 85.

[23] N. Bergman and F. Gustafsson. Three statistical batch algorithms for tracking manoeuvring targets. In Proceedings of the European Control Conference, pages 3082-3087, Karlsruhe, Germany, Aug. 1999. doi:10.23919/ECC.1999.7099799. Cited on page 93.

[24] D. Bertsekas. Linear Network Optimization: Algorithms and Codes. MIT Press, Cambridge, MA, USA, 1991. ISBN 978-0-262-02334-4. Cited on page 139.

[25] D. P. Bertsekas. The auction algorithm for assignment and other network flow problems: A tutorial. Interfaces, 20(4):133-149, July 1990. ISSN 0092-2102. Cited on page 139.

[26] A. Bhattacharyya. On a measure of divergence between two statistical populations defined by their probability distributions. Bulletin of Calcutta Mathematical Society, 35(1):99-109, 1943. Cited on page 144.

[27] G. Bianco, M. Ilieva, C. Veibäck, K. Öfjäll, A. Gadomska, G. Hendeby, M. Felsberg, F. Gustafsson, and S. Åkesson. Emlen-funnel experiments revisited: methods update for studying compass orientation in songbirds. Ecology and Evolution, 6(19):6930-6942, Sept. 2016. ISSN 2045-7758. doi:10.1002/ece3.2383. Cited on pages 8, 16, 19, 35, 49, 74, 75, 81, 91, 93, and 97.

[28] H. Bijl, J.-W. van Wingerden, T. B. Schön, and M. Verhaegen. Online sparse Gaussian process regression using FITC and PITC approximations. In Proceedings of the IFAC Symposium on System Identification, volume 48, pages 703-708, Beijing, China, Oct. 2015. doi:10.1016/j.ifacol.2015.12.212. Cited on pages 32 and 57.

[29] H. Bijl, T. B. Schön, J. van Wingerden, and M. Verhaegen. System identification through online sparse Gaussian process regression with input noise. IFAC Journal of Systems and Control, 2:1-11, Dec. 2017. doi:10.1016/j.ifacsc.2017.09.001. Cited on pages 32, 58, 60, and 129.

[30] S. S. Blackman. Multiple-target Tracking with Radar Applications. Artech House, Norwood, MA, USA, 1986. ISBN 978-0-89006-179-4. Cited on pages 5, 137, and 139 . 
[31] S. S. Blackman and R. Popoli. Design and Analysis of Modern Tracking Systems. Artech House, Norwood, MA, USA, 1999. ISBN 978-1-58053-006-4. Cited on pages $3,5,6,8,24,130,133,134,135,136,137,139,140,143$, and 150

[32] H. A. P. Blom. An efficient filter for abruptly changing systems. In Proceedings of the IEEE Conference on Decision and Control, pages 656-658, Las Vegas, NV, USA, Dec. 1984. doi:10.1109/CDC.1984.272089. Cited on pages 7,31 , and 93.

[33] B. J. Boom, P. X. Huang, C. Spampinato, S. Palazzo, J. He, C. Beyan, E. Beauxis-Aussalet, J. van Ossenbruggen, G. Nadarajan, J. Y. Chen-Burger, D. Giordano, L. Hardman, F.-P. Lin, and R. B. Fisher. Long-term underwater camera surveillance for monitoring and analysis of fish populations. In Proceedings of the Workshop on Visual Observation and Analysis of Animal and Insect Behavior, Tsukuba, Japan, Nov. 2012. Cited on page 17.

[34] J. Y. Bouguet. Camera calibration toolbox for Matlab. http:// wWw.vision.caltech.edu/bouguetj/calib_doc/, 2010. Accessed: 2016-10-17. Cited on page 65.

[35] M. D. Breitenstein, F. Reichlin, B. Leibe, E. Koller-Meier, and L. Van Gool. Online multiperson tracking-by-detection from a single, uncalibrated camera. IEEE Transactions on Pattern Analysis and Machine Intelligence, 33(9): 1820-1833, Sept. 2011. ISSN 0162-8828. doi:10.1109/TPAMI.2010.232. Cited on page 71 .

[36] E. F. Brekke and M. Chitre. The multiple hypothesis tracker derived from finite set statistics. In Proceedings of the International Conference on Information Fusion, Xi'an, China, July 2017. doi:10.23919/ICIF.2017.8009708. Cited on page 150 .

[37] M. Briers, A. Doucet, and S. Maskell. Smoothing algorithms for statespace models. Annals of the Institute of Statistical Mathematics, 62(1):61-89, Feb. 2010. ISSN 0020-3157. doi:10.1007/s10463-009-0236-2. Cited on page 86.

[38] C. Buragohain and S. Suri. Quantiles on streams. In Encyclopedia of Database Systems, pages 2235-2240. Springer US, 2009. ISBN 978-0-387-39940-9. Cited on pages 69,73 , and 74 .

[39] H. R. Byrd, C. J. Gilbert, and J. Nocedal. A trust region method based on interior point techniques for nonlinear programming. Mathematical Programming, 89(1):149-185, Nov. 2000. ISSN 0025-5610. doi:10.1007/PL00011391. Cited on pages 66, 67, and 69.

[40] E. J. Candès, X. Li, Y. Ma, and J. Wright. Robust principal component analysis? Journal of the Association for Computing Machinery, 58(3):11:111:37, May 2011. ISSN 0004-5411. doi:10.1145/1970392.1970395. Cited on page 17 . 
[41] D. A. Castanón. New assignment algorithms for data association. In Proceedings of SPIE's International Symposium on Optical Engineering and Photonics in Aerospace Sensing, volume 1698, pages 313-323, Orlando, FL, USA, Apr. 1992. doi:10.1117/12.139398. Cited on page 139.

[42] F. Ceragioli, G. Lindmark, C. Veibäck, N. Wahlström, M. Lindfors, and C. Altafini. A bounded confidence model that preserves the signs of the opinions. In Proceedings of the European Control Conference, pages 543-548, Aalborg, Denmark, June 2016. doi:10.1109/ECC.2016.78103410. Not cited.

[43] S. Challa, R. J. Evans, and X. Wang. A Bayesian solution and its approximations to out-of-sequence measurement problems. Information Fusion, 4(3): 185-199, Sept. 2003. ISSN 1566-2535. doi:10.1016/S1566-2535(03)00037-X. Cited on page 24 .

[44] C. B. Chang and M. Athans. State estimation for discrete systems with switching parameters. IEEE Transactions on Aerospace and Electronic Systems, 14(3):418-425, May 1978. ISSN 0018-9251. doi:10.1109/TAES.1978.308603. Cited on page 49 .

[45] B. Cheung, S. Davey, and D. Gray. PMHT with timing uncertainty. IEEE Transactions on Aerospace and Electronic Systems, 50(4):2960-2973, Oct. 2014. ISSN 0018-9251. doi:10.1109/TAES.2014.120315. Cited on pages 24, 117, and 118.

[46] S. Coraluppi, C. Carthel, P. Braca, and L. Millefiori. The mixed OrnsteinUhlenbeck process and context exploitation in multi-target tracking. In Proceedings of the International Conference on Information Fusion, pages 217224, Heidelberg, Germany, July 2016. Cited on page 31.

[47] S. Coraluppi, C. Carthel, and A. Coon. An MHT approach to multisensor passive sonar tracking. In Proceedings of the International Conference on Information Fusion, pages 480-487, Cambridge, UK, July 2018. doi:10.23919/ICIF.2018.8455402. Cited on page 24.

[48] I. J. Cox and S. L. Hingorani. An efficient implementation of Reid's multiple hypothesis tracking algorithm and its evaluation for the purpose of visual tracking. IEEE Transactions on Pattern Analysis and Machine Intelligence, 18(2):138-150, Feb. 1996. ISSN 0162-8828. doi:10.1109/34.481539. Cited on page 139.

[49] I. J. Cox and M. L. Miller. On finding ranked assignments with application to multitarget tracking and motion correspondence. IEEE Transactions on Aerospace and Electronic Systems, 31(1):486-489, Jan. 1995. ISSN 0018-9251. doi:10.1109/7.366332. Cited on page 139.

[50] D. F. Crouse, P. Willett, and Y. Bar-Shalom. Developing a real-time track display that operators do not hate. IEEE Transactions on Signal Processing, 59(7):3441-3447, July 2011. ISSN 1053-587X. doi:10.1109/TSP.2011.2135346. Cited on page 8 . 
[51] W. C. Davidon. Variable metric method for minimization. Technical Report ANL5990, Argonne National Laboratory, Argonne, IL, USA, 1959. Cited on page 107.

[52] M. P. Deisenroth, M. F. Huber, and U. D. Hanebeck. Analytic momentbased Gaussian process filtering. In Proceedings of the Annual International Conference on Machine Learning, pages 225-232, Montreal, QC, Canada, June 2009. doi:10.1145/1553374.1553403. Cited on page 32.

[53] A. P. Dempster, N. M. Laird, and D. B. Rubin. Maximum likelihood from incomplete data via the EM algorithm. Journal of the Royal Statistical Society. Series B (Methodological), 39(1):1-38, 1977. ISSN 0035-9246. Cited on pages 24, 26, 70, 93, and 117.

[54] F. Devernay and O. Faugeras. Straight lines have to be straight: Automatic calibration and removal of distortion from scenes of structured enviroments. Machine Vision and Applications, 13(1):14-24, Aug. 2001. ISSN 0932-8092. doi:10.1007/PL00013269. Cited on page 68.

[55] O. E. Drummond, D. A. Castanón, and M. S. Bellovin. Comparison of 2-D assignment algorithms for sparse, rectangular, floating point, cost matrices. Journal of the SDI Panels on Tracking, 4:81-97, Dec. 1990. Cited on page 139.

[56] J. E. Hopcroft and R. Tarjan. Algorithm 447: Efficient algorithms for graph manipulation. Communications of the ACM, 16(6):372-378, June 1973. ISSN 0001-0782. doi:10.1145/362248.362272. Cited on page 139.

[57] K. Eik. Review of experiences within ice and iceberg management. Journal of Navigation, 61(4):557-572, Oct. 2008. ISSN 0373-4633. doi:10.1017/S0373463308004839. Cited on page 27.

[58] K. Eik. Iceberg drift modelling and validation of applied metocean hindcast data. Cold Regions Science and Technology, 57(2-3):67-90, July 2009. ISSN 0165-232X. doi:10.1016/j.coldregions.2009.02.009. Cited on page 16.

[59] D. Ellis, E. Sommerlade, and I. Reid. Modelling pedestrian trajectory patterns with Gaussian processes. In Proceedings of the IEEE International Conference on Computer Vision Workshops, pages 1229-1234, Kyoto, Japan, Sept. 2009. doi:10.1109/ICCVW.2009.5457470. Cited on page 31.

[60] S. T. Emlen. Migration: orientation and navigation. In D. S. Farner and J. R. King, editors, Avian Biology, pages 129-219. Academic Press, Amsterdam, Netherlands, Aug. 1975. ISBN 978-0-12-249405-5. Cited on page 19.

[61] S. T. Emlen and J. T. Emlen. A technique for recording migratory orientation of captive birds. The Auk, 83(3):361-367, July 1966. doi:10.2307/4083048. Cited on page 19. 
[62] A. Flodell and C. Christensson. Wildlife surveillance using a UAV and thermal imagery. Master's thesis, Linköping University, Linköping, Sweden, June 2016. Cited on page 21.

[63] P.-E. Forssén. Low and Medium Level Vision using Channel Representations. Dissertation No. 858, Linköping University, Linköping, Sweden, Mar. 2004. Cited on page 74 .

[64] T. Fortmann, Y. Bar-Shalom, and M. Scheffe. Sonar tracking of multiple targets using joint probabilistic data association. IEEE Journal of Oceanic Engineering, 8(3):173-184, July 1983. ISSN 0364-9059. doi:10.1109/JOE.1983.1145560. Cited on page 143.

[65] N. Friedman and S. Russell. Image segmentation in video sequences: A probabilistic approach. In Proceedings of the Conference on Uncertainty in Artificial Intelligence, pages 175-181, Providence, Rhode Island, Aug. 1997. Cited on page 17.

[66] W. Friedman, S. Potter, E. Hutchins, C. Johnson, M. Krützen, and R. Connor. Three aerial technologies for recording subsurface behaviors among wild bottlenose dolphins (Tursiops sp.). In Proceedings of the Biennial Conference on Marine Mammals, Dunedin, New Zealand, Dec. 2013. Cited on page 17.

[67] R. Frigola, F. Lindsten, T. B. Schön, and C. E. Rasmussen. Bayesian inference and learning in Gaussian process state-space models with particle MCMC. In Proceedings of the International Conference on Neural Information Processing Systems, pages 3156-3164, Lake Tahoe, NV, USA, Dec. 2013. Cited on pages 1 and 32 .

[68] A. Gelb, J. F. Kasper, R. A. Nash, C. F. Price, and A. A. Sutherland, editors. Applied Optimal Estimation. MIT Press, Cambridge, MA, USA, 1974. ISBN 978-0-262-57048-0. Cited on page 38.

[69] A. E. Gelfand and A. F. M. Smith. Sampling-based approaches to calculating marginal densities. Journal of the American Statistical Association, 85 (410):398-409, June 1990. ISSN 0162-1459. doi:10.2307/22897760. Cited on pages 26 and 116 .

[70] A. Gelman, J. Carlin, H. Stern, D. Dunson, A. Vehtari, and D. Rubin. Bayesian Data Analysis. CRC Press, Boca Raton, FL, USA, third edition, 2014. ISBN 978-1-4398-4095-5. Cited on pages 52, 99, and 116.

[71] S. Geman and D. Geman. Stochastic relaxation, Gibbs distributions, and the Bayesian restoration of images. IEEE Transactions on Pattern Analysis and Machine Intelligence, 6(6):721-741, Nov. 1984. ISSN 0162-8828. doi:10.1109/TPAMI.1984.4767596. Cited on pages 26, 116, and 118.

[72] K. Gilholm and D. Salmond. Spatial distribution model for tracking extended objects. IEE Proceedings Radar, Sonar and Navigation, 152(5):364-371, Oct. 2005. ISSN 1350-2395. doi:10.1049/ip-rsn:20045114. Cited on page 52. 
[73] W. R. Gilks, N. G. Best, and K. K. C. Tan. Adaptive rejection Metropolis sampling within Gibbs sampling. Journal of the Royal Statistical Society. Series C (Applied Statistics), 44(4):455-472, $1995 . \quad$ ISSN 0035-9254. doi:10.2307/2986138. Cited on page 116 .

[74] L. F. Gonzalez, G. A. Montes, E. Puig, S. Johnson, K. Mengersen, and K. J. Gaston. Unmanned aerial vehicles (UAVs) and artificial intelligence revolutionizing wildlife monitoring and conservation. Sensors, 16(1):97114, Jan. 2016. ISSN 1424-8220. doi:10.3390/s16010097. Cited on page 21.

[75] R. C. Gonzalez and R. E. Woods. Digital Image Processing. Pearson Prentice Hall, Upper Saddle River, NJ, USA, third edition, 2008. ISBN 978-0-13168728-8. Cited on pages 6, 69, 71, 74, 75, and 77.

[76] N. J. Gordon, D. J. Salmond, and A. F. M. Smith. Novel approach to nonlinear/non-Gaussian Bayesian state estimation. IEE Proceedings F Radar and Signal Processing, 140(2):107-113, Apr. 1993. ISSN 0956-375X. doi:10.1049/ip-f-2.1993.00150. Cited on pages 7 and 85.

[77] K. Granström and U. Orguner. A PHD filter for tracking multiple extended targets using random matrices. IEEE Transactions on Signal Processing, 60 (11):5657-5671, Nov. 2012. ISSN 1053-587X. doi:10.1109/TSP.2012.2212888. Cited on pages 52 and 101.

[78] K. Granström, C. Lundquist, and U. Orguner. Tracking rectangular and elliptical extended targets using laser measurements. In Proceedings of the International Conference on Information Fusion, Chicago, IL, USA, July 2011. Cited on page 52.

[79] M. S. Grewal and A. P. Andrew. Kalman Filtering: Theory and Practice. Prentice Hall, Englewood Cliffs, New Jersey, USA, 1993. ISBN 978-0-13211335-9. Cited on pages 7 and 81.

[80] S. Gunnarsson, Y. Jung, C. Veibäck, and T. Glad. IO (implement and operate) first - an alternative way to approach the automatic control subject. In Proceedings of Utvecklingskonferensen för Sveriges ingenjörsutbildningar, pages 163-167, Uppsala, Sweden, Feb. 2016. Not cited.

[81] F. Gustafsson. Statistical Sensor Fusion. Studentlitteratur, Lund, Sweden, second edition, 2012. ISBN 978-91-44-07732-1. Cited on pages 7, 37, 86, 87, 91, and 166.

[82] F. Gustafsson, F. Gunnarsson, N. Bergman, U. Forssell, J. Jansson, R. Karlsson, and P. J. Nordlund. Particle filters for positioning, navigation, and tracking. IEEE Transactions on Signal Processing, 50(2):425-437, Feb. 2002. ISSN 1053-587X. doi:10.1109/78.978396. Cited on pages 31, 85, and 86.

[83] A. Guttman. R-trees: A dynamic index structure for spatial searching. In Proceedings of the ACM SIGMOD International Conference on 
Management of Data, pages 47-57, Boston, MA, USA, June 1984. doi:10.1145/602259.602266. Cited on page 139 .

[84] J. Hannah. A geometric approach to determinants. The American Mathematical Monthly, 103(5):401-409, May 1996. ISSN 0002-9890. doi:10.2307/2974931. Cited on page 168 .

[85] L.-A. Hansson and S. Åkesson, editors. Animal Movement Across Scales. Oxford University Press, Oxford, UK, 2014. ISBN 978-0-19-967718-4. Cited on page 19 .

[86] R. Hartley and A. Zisserman. Multiple View Geometry in Computer Vision. Cambridge University Press, Cambridge, UK, second edition, 2003. ISBN 978-0-521-54051-3. Cited on page 63.

[87] J. Haugen. Autonomous Aerial Ice Observation. Dissertation No. 2017:291, Norwegian University of Science and Technology, Trondheim, Norway, Oct. 2014. Cited on page 27.

[88] D. L. Herzing. Vocalizations and associated underwater behavior of freeranging Atlantic spotted dolphins, Stenella frontalis and bottlenose dolphins, Tursiops truncatus. Aquatic Mammals, 22(2):61-79, Jan. 1996. ISSN 0167-5427. doi:10.12966/abc.02.02.2015. Cited on page 17.

[89] S. Hexeberg, A. L. Flåten, B. O. H. Eriksen, and E. F. Brekke. AIS-based vessel trajectory prediction. In Proceedings of the International Conference on Information Fusion, Xi'an, China, July 2017. doi:10.23919/ICIF.2017.8009762. Cited on page 31.

[90] R. D. Hilton, D. A. Martin, and W. D. Blair. Tracking with time-delayed data in multisensor systems. Technical Report TR-93/351, NSWCDD, Dahlgren, VA, USA, 1993. Cited on pages 1 and 24.

[91] R. A. Horn and C. R. Johnson. Matrix Analysis. Cambridge University Press, New York, NY, USA, 2nd edition, 2013. ISBN 978-0-521-54823-6. Cited on page 164 .

[92] M. F. Huber. Recursive Gaussian process: On-line regression and learning. Pattern Recognition Letters, 45:85-91, Aug. 2014. ISSN 0167-8655. doi:10.1016/j.patrec.2014.03.004. Cited on pages 32, 57, and 60.

[93] M. Israel. A UAV-based roe deer fawn detection system. In Proceedings of the International Conference on Unmanned Aerial Vehicle in Geomatics, volume 3822, pages 51-55, Zurich, Switzerland, Sept. 2011. doi:10.5194/isprsarchives-XXXVIII-1-C22-51-2011. Cited on page 21.

[94] A. Jazwinski. Stochastic Processes and Filtering Theory, volume 64. Academic Press, New York, NY, USA, 1970. ISBN 978-0-486-46274-5. Cited on page 38. 
[95] T. A. Johansen and T. Perez. Unmanned aerial surveillance system for hazard collision avoidance in autonomous shipping. In Proceedings of the International Conference on Unmanned Aircraft Systems, pages 1056-1065, Arlington, VA, USA, June 2016. doi:10.1109/ICUAS.2016.7502542. Cited on page 27.

[96] G. P. Jones IV, L. G. Pearlstine, and H. F. Percival. An assessment of small unmanned aerial vehicles for wildlife research. Wildlife Society Bulletin, 34(3):750-758, Oct. 2006. ISSN 1938-5463. doi:10.2193/0091-7648(2006)34[750:AAOSUA]2.0.CO;2. Cited on page 21.

[97] R. Jonker and A. Volgenant. A shortest augmenting path algorithm for dense and sparse linear assignment problems. Computing, 38(4):325-340, Dec. 1987. ISSN 1436-5057. doi:10.1007/BF02278710. Cited on page 139.

[98] S. J. Julier and J. K. Uhlmann. Unscented filtering and nonlinear estimation. Proceedings of the IEEE, 92(3):401-422, Mar. 2004. ISSN 0018-9219. doi:10.1109/JPROC.2003.823141. Cited on pages 7 and 84 .

[99] S. J. Julier and J. K. Uhlmann. Fusion of time delayed measurements with uncertain time delays. In Proceedings of the American Control Conference, pages 4028-4033, Portland, OR, USA, June 2005. doi:10.1109/ ACC.2005.1470607. Cited on page 24 .

[100] S. J. Julier, J. K. Uhlmann, and H. F. Durrant-Whyte. A new method for the nonlinear transformation of means and covariances in filters and estimators. IEEE Transactions on Automatic Control, 45(3):477-482, Mar. 2000. ISSN 0018-9286. doi:10.1109/9.847726. Cited on pages 7 and 78.

[101] R. E. Kalman. A new approach to linear filtering and prediction problems. Journal of Basic Engineering, 82(1):35-45, Mar. 1960. doi:10.1115/1.3662552. Cited on pages $7,81,83$, and 123.

[102] J. Karnowski, E. Hutchins, and C. Johnson. Dolphin detection and tracking. In Proceedings of the IEEE Winter Applications and Computer Vision Workshops, pages 51-56, Waikoloa Beach, HI, USA, Jan. 2015. doi:10.1109/WACVW.2015.10. Cited on page 17.

[103] S. M. Kay. Fundamentals of Statistical Signal Processing: Estimation Theory. Prentice Hall, Englewood Cliffs, NJ, USA, 1993. ISBN 978-0-13-345711-7. Cited on pages 7, 81, 103, 104, 107, 112, and 119.

[104] O. Khatib. Real-time obstacle avoidance for manipulators and mobile robots. The International Journal of Robotics Research, 5(1):90-98, Mar. 1986. doi:10.1177/027836498600500106. Cited on page 42.

[105] K. Kim, D. Lee, and I. Essa. Gaussian process regression flow for analysis of motion trajectories. In Proceedings of the International Conference on Computer Vision, pages 1164-1171, Barcelona, Spain, Nov. 2011. doi:10.1109/ICCV.2011.6126365. Cited on page 31. 
[106] G. Kitagawa. Non-Gaussian state-space modeling of nonstationary time series. Journal of the American Statistical Association, 82(400):1032-1041, Dec. 1987. ISSN 0162-1459. doi:10.2307/2289375. Cited on page 82.

[107] J. W. Koch. Bayesian approach to extended object and cluster tracking using random matrices. IEEE Transactions on Aerospace and Electronic Systems, 44(3):1042-1059, July 2008. ISSN 0018-9251. doi:10.1109/TAES.2008.4655362. Cited on pages 5, 52, 99, 100, and 101.

[108] M. Kok and A. Solin. Scalable magnetic field SLAM in 3D using Gaussian process maps. In Proceedings of the International Conference on Information Fusion, pages 1353-1360, Cambridge, UK, July 2018. doi:10.23919/ICIF.2018.8455789. Cited on page 32.

[109] H. W. Kuhn. The Hungarian method for the assignment problem. Naval Research Logistics Quarterly, 2(1-2):83-97, Mar. 1955. doi:10.1002/nav.3800020109. Cited on page 139.

[110] S. Kullback and R. A. Leibler. On information and sufficiency. The Annals of Mathematical Statistics, 22(1):79-86, Mar. 1951. doi:10.1214/aoms/1177729694. Cited on page 91.

[111] T. D. Larsen, N. A. Andersen, O. Ravn, and N. K. Poulsen. Incorporation of time delayed measurements in a discrete-time Kalman filter. In Proceedings of the IEEE Conference on Decision and Control, volume 4, pages 3972-3977, Tampa, FL, USA, Dec. 1998. doi:10.1109/CDC.1998.761918. Cited on page 24.

[112] F. S. Leira. Object Detection and Tracking With UAVs: A Framework for UAV Object Detection and Tracking Using a Thermal Imaging Camera. Dissertation No. 2017:1, Norwegian University of Science and Technology, Trondheim, Norway, Jan. 2017. Cited on page 27.

[113] M. Levedahl. Performance comparison of 2D assignment algorithms for assigning truth objects to measured tracks. In Proceedings of SPIE's International Symposium on Aerospace and Defense Sensing, Simulations and Controls, volume 4048, Orlando, FL, USA, Apr. 2000. doi:10.1117/12.392018. Cited on page 139 .

[114] R. X. Li and V. P. Jilkov. Survey of maneuvering target tracking. Part I: Dynamic models. IEEE Transactions on Aerospace and Electronic Systems, 39 (4):1333-1364, Oct. 2003. ISSN 0018-9251. doi:10.1109/TAES.2003.1261132. Cited on pages $7,40,41,45$, and 130 .

[115] L. Ljung. System Identification: Theory for the User. Prentice Hall, Upper Saddle River, NJ, USA, second edition, 1999. ISBN 978-0-13-656695-3. Cited on pages 1,36 , and 131 . 
[116] A. Logothetis and V. Krishnamurthy. Expectation maximization algorithms for MAP estimation of jump Markov linear systems. IEEE Transactions on Signal Processing, 47(8):2139-2156, Aug. 1999. ISSN 1053-587X. doi:10.1109/78.774753. Cited on page 93.

[117] Y. Ma, S. Soatto, J. Kosecka, and S. Sastry. An Invitation to 3-D Vision. Springer, New York, NY, USA, 2004. ISBN 978-0-387-00893-6. Cited on pages 63, 64, and 65 .

[118] J. B. MacQueen. Some methods for classification and analysis of multivariate observations. In Proceedings of the Berkeley Symposium on Mathematical Statistics and Probability, volume 1, pages 281-297, Berkeley, CA, USA, 1967. Cited on page 78.

[119] R. Mahler. A brief survey of advances in random-set fusion. In Proceedings of the International Conference on Control, Automation and Information Sciences, pages 62-67, Changshu, China, Oct. 2015. doi:10.1109/ICCAIS.2015.7338726. Cited on page 150 .

[120] R. P. S. Mahler. Multitarget Bayes filtering via first-order multitarget moments. IEEE Transactions on Aerospace and Electronic Systems, 39(4):11521178, Oct. 2003. ISSN 0018-9251. doi:10.1109/TAES.2003.1261119. Cited on page 150 .

[121] R. P. S. Mahler. Statistical Multisource-Multitarget Information Fusion. Artech House, Norwood, MA, USA, 2007. ISBN 978-1-59693-092-6. Cited on pages $5,133,150$, and 151.

[122] M. Mallick and B. La Scala. Comparison of single-point and twopoint difference track initiation algorithms using position measurements. Acta Automatica Sinica, 34(3):258-265, Mar. 2008. ISSN 1874-1029. doi:10.3724/SP.J.1004.2008.00258. Cited on page 41.

[123] M. Mallick, S. Coraluppi, and C. Carthel. Advances in asynchronous and decentralized estimation. In Proceedings of the IEEE Aerospace Conference, volume 4, pages 1873-1888, Big Sky, MT, USA, Mar. 2001. doi:10.1109/AERO.2001.931505. Cited on pages 1 and 24 .

[124] J. Martin, H. H. Edwards, M. A. Burgess, H. F. Percival, D. E. Fagan, B. E. Gardner, J. G. Ortega-Ortiz, P. G. Ifju, B. S. Evers, and T. J. Rambo. Estimating distribution of hidden objects with drones: From tennis balls to manatees. PLoS ONE, 7(6), June 2012. doi:10.1371/journal.pone.0038882. Cited on page 21.

[125] A. McHutchon and C. E. Rasmussen. Gaussian process training with input noise. In Proceedings of the International Conference on Neural Information Processing Systems, pages 1341-1349, Granada, Spain, Dec. 2011. Cited on page 60 . 
[126] N. Metropolis, A. Rosenbluth, M. Rosenbluth, A. Teller, and E. Teller. Equation of state calculations by fast computing machines. The Journal of Chemical Physics, 21(6):1087-1092, June 1953. ISSN 0021-9606. doi:10.1063/1.1699114. Cited on page 116.

[127] G. Meurant. A review on the inverse of symmetric tridiagonal and block tridiagonal matrices. SIAM Journal on Matrix Analysis and Applications, 13 (3):707-728, 1992. doi:10.1137/0613045. Cited on pages 88 and 166.

[128] L. M. Millefiori, P. Braca, K. Bryan, and P. Willett. Modeling vessel kinematics using a stochastic mean-reverting process for long-term prediction. IEEE Transactions on Aerospace and Electronic Systems, 52(5):2313-2330, Oct. 2016. ISSN 0018-9251. doi:10.1109/TAES.2016.150596. Cited on page 31.

[129] M. L. Miller, H. S. Stone, and I. J. Cox. Optimizing Murty's ranked assignment method. IEEE Transactions on Aerospace and Electronic Systems, 33(3): 851-862, July 1997. ISSN 0018-9251. doi:10.1109/7.599256. Cited on pages 139 and 153.

[130] M. Morelande. Linear filtering with timing uncertainty. In Proceedings of the International Conference on Information Fusion, Cologne, Germany, June 2008. Cited on page 24.

[131] H. Mouritsen, G. Feenders, M. Liedvogel, and W. Kropp. Migratory birds use head scans to detect the direction of the earth's magnetic field. Current Biology, 14(21):1946-1949, Nov. 2004. ISSN 0960-9822. doi:10.1016/j.cub.2004.10.025. Cited on page 19.

[132] R. Muheim, I. Henshaw, S. Sjöberg, and M. E. Deutschlander. Birdoritrack: a new video-tracking program for orientation research with migratory birds. Journal of Field Ornithology, 85(1):91-105, Mar. 2014. ISSN 1557-9263. doi:10.1111/jofo.12053. Cited on page 19.

[133] M. Mulero-Pázmány, R. Stolper, L. D. van Essen, J. J. Negro, and T. Sassen. Remotely piloted aircraft systems as a rhinoceros anti-poaching tool in africa. PLoS ONE, 9(1), Jan. 2014. doi:10.1371/journal.pone.0083873. Cited on page 21.

[134] K. G. Murty. An algorithm for ranking all the assignments in order of increasing cost. Operations Research, 16(3):682-687, May 1968. doi:10.1287/opre.16.3.682. Cited on pages 139 and 153.

[135] J. Nilsson, B. Bernhardsson, and B. Wittenmark. Stochastic analysis and control of real-time systems with random time delays. Automatica, 34 (1):57-64, Jan. 1998. ISSN 0005-1098. doi:10.1016/S0005-1098(97)00170-2. Cited on page 24 . 
[136] J. O. Nilsson and P. Händel. Time synchronization and temporal ordering of asynchronous sensor measurements of a multi-sensor navigation system. In Proceedings of the IEEE/ION Position, Location and Navigation Symposium, pages 897-902, Indian Wells, CA, USA, May 2010. doi:10.1109/PLANS.2010.5507193. Cited on page 24.

[137] J. O. Nilsson, I. Skog, and P. Händel. Joint state and measurement time-delay estimation of nonlinear state space systems. In Proceedings of the International Conference on Information Science, Signal Processing and their Applications, pages 324-328, Kuala Lumpur, Malaysia, May 2010. doi:10.1109/ISSPA.2010.5605534. Cited on page 24.

[138] J. Nocedal and S. Wright. Numerical Optimization. Springer, New York, NY, USA, second edition, 2006. ISBN 978-0-387-30303-1. Cited on pages 66, 85, 112 , and 116.

[139] J. Olofsson, E. Brekke, T. I. Fossen, and T. A. Johansen. Spatially indexed clustering for scalable tracking of remotely sensed drift ice. In Proceedings of the IEEE Aerospace Conference, Big Sky, MT, USA, Mar. 2017. doi:10.1109/AERO.2017.7943670. Cited on page 27.

[140] J. Olofsson, A. Lindahl Flåten, and C. Veibäck. Gaussian field current estimation from drift sea ice tracking with the labeled multi-Bernoulli filter. In Proceedings of the OCEANS Conference, Anchorage, AK, USA, Sept. 2017. Cited on page 29.

[141] J. Olofsson, C. Veibäck, and G. Hendeby. Sea ice tracking with a spatially indexed labeled multi-Bernoulli filter. In Proceedings of the International Conference on Information Fusion, pages 376-383, Xi'an, China, July 2017. doi:10.23919/ICIF.2017.80096720. Cited on pages 8, 12, 16, 29, 30, and 157.

[142] J. Olofsson, C. Veibäck, G. Hendeby, and T. A. Johansen. Outline of a system for integrated adaptive ice tracking and multi-agent path planning. In Proceedings of the Workshop on Research, Education and Development of Unmanned Aerial Systems, Linköping, Sweden, Oct. 2017. doi:10.1109/REDUAS.2017.8101636. Cited on page 29.

[143] N. Otsu. A threshold selection method from gray-level histograms. IEEE Transactions on Systems, Man, and Cybernetics, 9(1):62-66, Jan. 1979. ISSN 0018-9472. doi:10.1109/TSMC.1979.4310076. Cited on page 77.

[144] C. C. Paige and M. A. Saunders. Least squares estimation of discrete linear dynamic systems using orthogonal transformations. SIAM Journal on Numerical Analysis, 14(2):180-193, Apr. 1977. doi:10.1137/0714012. Cited on page 87 .

[145] G. Pallotta, S. Horn, P. Braca, and K. Bryan. Context-enhanced vessel prediction based on Ornstein-Uhlenbeck processes using historical AIS traffic 
patterns: Real-world experimental results. In Proceedings of the International Conference on Information Fusion, Salamanca, Spain, July 2014. Cited on page 31.

[146] C. R. Pedersen, L. R. Nielsen, and K. A. Andersen. An algorithm for ranking assignments using reoptimization. Computers $\mathcal{E}$ Operations Research, 35 (11):3714-3726, Nov. 2008. ISSN 0305-0548. doi:10.1016/j.cor.2007.04.008. Cited on page 139 .

[147] P. W. Power and J. A. Schoonees. Understanding background mixture models for foreground segmentation. In Proceedings of Image and Vision Computing New Zealand, pages 267-271, Auckland, New Zealand, Nov. 2002. Cited on pages 69 and 70.

[148] J. Quiñonero Candela and C. E. Rasmussen. A unifying view of sparse approximate gaussian process regression. Journal of Machine Learning Research, 6:1939-1959, Dec. 2005. ISSN 1532-4435. Cited on pages 31, 32, 58, and 59 .

[149] L. RÅde and B. Westergren. Mathematics Handbook for Science and Engineering. Studentlitteratur, Lund, Sweden, fifth edition, 2004. ISBN 978-91-4403109-5. Cited on pages 161 and 162.

[150] C. Randell, F. Ralph, D. Power, and P. Stuckey. SS: Canadian: Atlantic development; technological advances to assess, manage and reduce ice risk in northern developments. In Proceedings of the Offshore Technology Conference, Houston, TX, USA, May 2009. doi:10.4043/OTC-20264-MS. Cited on page 16.

[151] C. E. Rasmussen and C. K. I. Williams. Gaussian Processes for Machine Learning. Adaptive Computation and Machine Learning. MIT Press, Cambridge, MA, USA, 2006. ISBN 978-0-262-18253-9. Cited on pages 31, 57, and 60 .

[152] H. E. Rauch, F. Tung, and C. T. Striebel. Maximum likelihood estimates of linear dynamic systems. AIAA Journal, 3(8):1445-1450, Aug. 1965. doi:10.2514/3.3166. Cited on pages 7, 87, and 102.

[153] D. B. Reid. An algorithm for tracking multiple targets. IEEE Transactions on Automatic Control, 24(6):843-854, Dec. 1979. ISSN 0018-9286. doi:10.1109/TAC.1979.1102177. Cited on pages 6, 140, and 150.

[154] S. Reuter, B.-T. Vo, B.-N. Vo, and K. Dietmayer. The labeled multi-Bernoulli filter. IEEE Transactions on Signal Processing, 62(12):3246-3260, May 2014. ISSN 1070-9908. doi:10.1109/TSP.2014.2323064. Cited on pages 6, 29, 150, 151, 152, and 154.

[155] C. Röver and T. Friede. Discrete approximation of a mixture distribution via restricted divergence. Journal of Computational and Graphical Statistics, 26(1):217-222, 2017. doi:10.1080/10618600.2016.1276840. Cited on page 103. 
[156] D. B. Rubin. Using the SIR algorithm to simulate posterior distributions. In M. H. Bernardo, K. M. DeGroot, D. V. Lindley, and A. F. M. Smith, editors, Bayesian Statistics 3, pages 395-402. Oxford University Press, 1988. Cited on page 116.

[157] W. J. Rugh. Linear system theory. Prentice Hall, Upper Saddle River, NJ, 2nd edition, 1996. ISBN 978-0-13-441205-4. Cited on page 46.

[158] A. R. Runnalls. A Kullback-Leibler approach to Gaussian mixture reduction. IEEE Transactions on Aerospace and Electronic Systems, 43(3):989-999, July 2007. ISSN 0018-9251. doi:10.1109/TAES.2007.43835880. Cited on page 144 .

[159] S. Särkkä. Bayesian Filtering and Smoothing. Cambridge University Press, Cambridge, United Kingdom, 2013. ISBN 978-1-107-03065-7. Cited on pages $7,36,57,81,83,86,88,102$, and 123 .

[160] L. Schenato. Optimal estimation in networked control systems subject to random delay and packet loss. In Proceedings of the IEEE Conference on Decision and Control, pages 5615-5620, San Diego, CA, USA, Dec. 2006. doi:10.1109/CDC.2006.377700. Cited on page 24.

[161] L. Shi, L. Xie, and R. M. Murray. Kalman filtering over a packet-delaying network: A probabilistic approach. Automatica, 45(9):2134-2140, Sept. 2009. doi:10.1016/j.automatica.2009.05.018. Cited on page 24 .

[162] Smart Savannahs. http://wildlifesecurity.se/ smart-savannahs/, 2016. Accessed: 2016-10-17. Cited on pages 16,21 , and 23.

[163] A. F. M. Smith and A. E. Gelfand. Bayesian statistics without tears: A sampling-resampling perspective. The American Statistician, 46(2):84-88, May 1992. ISSN 0003-1305. doi:10.2307/2684170. Cited on page 116.

[164] G. L. Smith, S. F. Schmidt, and L. A. McGee. Application of statistical filter theory to the optimal estimation of position and velocity on board a circumlunar vehicle. Technical Report NASA TR R-135, National Aeronautics and Space Administration, Washington D. C., USA, 1962. Cited on pages $7,32,81,84$, and 123 .

[165] D. Snyder and P. Fishman. How to track a swarm of fireflies by observing their flashes (corresp.). IEEE Transactions on Information Theory, 21(6):692695, Nov. 1975. ISSN 0018-9448. doi:10.1109/TIT.1975.1055455. Cited on page 24 .

[166] A. Solin, M. Kok, N. Wahlström, T. B. Schön, and S. Särkkä. Modeling and interpolation of the ambient magnetic field by Gaussian processes. IEEE Transactions on Robotics, 34:1112-1127, Aug. 2018. doi:10.1109/TRO.2018.2830326. Cited on page 59. 
[167] C. Stauffer and W. E. L. Grimson. Adaptive background mixture models for real-time tracking. In Proceedings of the IEEE Computer Society Conference on Computer Vision and Pattern Recognition, volume 2, pages 2246-2252, Ft. Collins, CO, USA, June 1999. doi:10.1109/CVPR.1999.784637. Cited on pages 69 and 70 .

[168] L. D. Stone, R. L. Streit, T. L. Corwin, and K. L. Bell. Bayesian Multiple Target Tracking. Artech House, Norwood, MA, USA, second edition, 2014. ISBN 978-1-60807-553-9. Cited on page 133.

[169] A. J. Storkey. Truncated covariance matrices and Toeplitz methods in Gaussian processes. In Proceedings of the International Conference on Artificial Neural Networks, volume 1, pages 55-60, Edinburgh, UK, Sept. 1999. doi:10.1049/cp:19991084. Cited on page 128.

[170] R. Tarjan. Depth-first search and linear graph algorithms. SIAM Journal on Computing, 1(2):146-160, 1972. doi:10.1137/0201010. Cited on page 139.

[171] L. Taycher, J. W. Fischer III, and T. Darrel. Incorporating object tracking feedback into background maintenance framework. In Proceedings of the IEEE Workshops on Application of Computer Vision, volume 2, pages 120-125, Breckenridge, CO, USA, Jan. 2005. doi:10.1109/ACVMOT.2005.63. Cited on page 71 .

[172] S. C. Thomopoulos and L. Zhang. Decentralized filtering with random sampling and delay. Information Sciences, 81(1-2):117-131, Nov. 1994. ISSN 0020-0255. doi:10.1016/0020-0255(94)90093-0. Cited on page 24.

[173] M. Tiger and F. Heintz. Online sparse Gaussian process regression for trajectory modeling. In Proceedings of the International Conference on Information Fusion, pages 782-791, Washington D.C., USA, July 2015. Cited on page 31.

[174] Q. Tran and J. Firl. Modelling of traffic situations at urban intersections with probabilistic non-parametric regression. In Proceedings of the IEEE Intelligent Vehicles Symposium, pages 334-339, Gold Coast, QLD, Australia, June 2013. doi:10.1109/IVS.2013.6629491. Cited on page 31.

[175] J. Tugnait. Adaptive estimation and identification for discrete systems with markov jump parameters. IEEE Transactions on Automatic Control, 27 (5):1054-1065, Oct. 1982. ISSN 0018-9286. doi:10.1109/TAC.1982.1103061. Cited on page 7 .

[176] R. Turner, M. Deisenroth, and C. Rasmussen. State-space inference and learning with Gaussian processes. In Proceedings of the International Conference on Artificial Intelligence and Statistics, pages 868-875, Chia Laguna Resort, Sardinia, Italy, May 2010. Cited on page 32.

[177] R. van der Merwe, E. A. Wan, and S. I. Julier. Sigma-point Kalman filters for nonlinear estimation and sensor-fusion: Applications to integrated 
navigation. In Proceedings of the AIAA Guidance, Navigation and Control Conference, Providence, RI, USA, Aug. 2004. doi:10.2514/6.2004-5120. Cited on page 24.

[178] J. C. van Gemert, C. R. Verschoor, P. Mettes, K. Epema, L. P. Koh, and S. Wich. Nature conservation drones for automatic localization and counting of animals. In Proceedings of the European Conference on Computer Vision, pages 255-270, Zurich, Switzerland, Sept. 2014. doi:10.1007/978-3-31916178-5_17. Cited on page 21.

[179] L. van Zonneveld. The importance of acoustic enrichment for bottlenose dolphins (Tursiops truncatus) in human care. Master's thesis, Linköping University, Linköping, Sweden, Mar. 2015. Cited on page 17.

[180] C. Veibäck. Tracking of Animals Using Airborne Cameras. Licentiate's Thesis No. 1761, Linköping University, Nov. 2016. Cited on pages 16, 44, and 90.

[181] C. Veibäck, G. Hendeby, and F. Gustafsson. Tracking of dolphins in a basin using a constrained motion model. In Proceedings of the International Conference on Information Fusion, pages 1330-1337, Washington D. C., USA, July 2015 . Cited on pages $8,16,17,35,42,44,45,48,63,72,73,133,143$, $145,146,147,148$, and 149.

[182] C. Veibäck, G. Hendeby, and F. Gustafsson. On fusion of sensor measurements and observation with uncertain timestamp for target tracking. In Proceedings of the International Conference on Information Fusion, pages 12681275, Heidelberg, Germany, July 2016. Cited on pages 8, 16, 25, 35, 53, 81, $102,105,106,108,109,114$, and 116.

[183] C. Veibäck, G. Hendeby, and F. Gustafsson. Uncertain timestamps in linear state estimation. IEEE Transactions on Aerospace and Electronic Systems, accepted for publication August 21, 2018. ISSN 0018-9251. doi:10.1109/TAES.2018.2869648. Cited on pages 8, 16, 26, 53, 89, 102, 120, 121 , and 166.

[184] C. Veibäck, J. Olofsson, T. R. Lauknes, and G. Hendeby. Learning target dynamics while tracking using Gaussian processes. IEEE Transactions on Aerospace and Electronic Systems, submitted June 13, 2018. ISSN 0018-9251. Cited on pages $8,16,29$, and 57 .

[185] C. Vermeulen, P. Lejeune, J. Lisein, P. Sawadogo, and P. Bouché. Unmanned aerial survey of elephants. PLoS ONE, 8(2), Feb. 2013. doi:10.1371/journal.pone.0054700. Cited on page 21.

[186] B. N. Vo and W. K. Ma. The Gaussian mixture probability hypothesis density filter. IEEE Transactions on Signal Processing, 54(11):4091-4104, Nov. 2006. ISSN 1053-587X. doi:10.1109/TSP.2006.881190. Cited on page 6.

[187] B.-N. Vo, B.-T. Vo, and D. Phung. Labeled random finite sets and the Bayes multi-target tracking filter. IEEE Transactions on Signal Processing, 62 
(24):6554-6567, Dec. 2014. ISSN 1053-587X. doi:10.1109/TSP.2014.2364014. Cited on page 150 .

[188] B. N. Vo, B. T. Vo, and H. G. Hoang. An efficient implementation of the generalized labeled multi-Bernoulli filter. IEEE Transactions on Signal Processing, 65(8):1975-1987, Apr. 2017. ISSN 1053-587X. doi:10.1109/TSP.2016.2641392. Cited on pages 139 and 153.

[189] B. T. Vo and B. N. Vo. Labeled random finite sets and multi-object conjugate priors. IEEE Transactions on Signal Processing, 61(13):3460-3475, July 2013. ISSN 1053-587X. doi:10.1109/TSP.2013.2259822. Cited on pages 6, 150, 151, and 152.

[190] D. Voytenko, T. H. Dixon, M. E. Luther, C. Lembke, I. M. Howat, and S. de la Peña. Observations of inertial currents in a lagoon in southeastern Iceland using terrestrial radar interferometry and automated iceberg tracking. Computers $\mathcal{E}$ Geosciences, 82:23-30, Sept. 2015. ISSN 0098-3004. doi:10.1016/j.cageo.2015.05.012. Cited on page 29.

[191] N. Wahlström and E. Özkan. Extended target tracking using Gaussian processes. IEEE Transactions on Signal Processing, 63(16):4165-4178, Aug. 2015. ISSN 1053-587X. doi:10.1109/TSP.2015.2424194. Cited on pages 32, 52,59 , and 60 .

[192] N. Wahlström, P. Axelsson, and F. Gustafsson. Discretizing stochastic dynamical systems using Lyapunov equations. In Proceedings of the World Congress of the International Federation of Automatic Control, pages 37263731, Cape Town, South Africa, Aug. 2014. doi:10.3182/20140824-6-ZA1003.02157. Cited on pages 38 and 39.

[193] A. Wald. Sequential tests of statistical hypotheses. The Annals of Mathematical Statistics, 16(2):117-186, June 1945. ISSN 0003-4851. doi:10.1214/aoms/1177731118. Cited on page 136.

[194] H. G. Wallraff and O. G. Gelderloos. Experiments on migratory orientation of birds with simulated stellar sky and geomagnetic field: Method and preliminary results. In Proceedings of a Symposium at Falsterbo, pages 207215, Falsterbo, Sweden, Oct. 1977. doi:10.2307/3543480. Cited on page 19.

[195] A. C. Watts, J. H. Perry, S. E. Smith, M. A. Burgess, B. E. Wilkinson, Z. Szantoi, P. G. Ifju, and H. F. Percival. Small unmanned aircraft systems for low-altitude aerial surveys. Journal of Wildlife Management, 74(7):1614-1619, Sept. 2010. ISSN 0022-541X. doi:10.2193/2009-425. Cited on page 21.

[196] J. R. Werthmann. Step-by-step description of a computationally efficient version of multiple hypothesis tracking. In Proceedings of SPIE's International Symposium on Optical Engineering and Photonics in Aerospace Sensing, volume 1698, pages 288-300, Orlando, FL, USA, Apr. 1992. doi:10.1117/12.139379. Cited on page 140 . 
[197] J. L. Williams. Marginal multi-Bernoulli filters: RFS derivation of MHT, JIPDA, and association-based member. IEEE Transactions on Aerospace and Electronic Systems, 51(3):1664-1687, July 2015. ISSN 0018-9251. doi:10.1109/TAES.2015.130550. Cited on pages 6, 29, and 154.

[198] W. Wiltschko and R. Wiltschko. Magnetic Orientation and Celestial Cues in Migratory Orientation, pages 16-37. Birkhäuser Basel, Basel, Switzerland, 1991. ISBN 978-3-0348-7208-9. Cited on page 19.

[199] K. Zhang, X. R. Li, and Y. Zhu. Optimal update with out-of-sequence measurements. IEEE Transactions on Signal Processing, 53(6):1992-2004, June 2005. doi:10.1109/TSP.2005.8478300. Cited on page 24 .

[200] K. Zhang, L. Zhang, and M.-H. Yang. Real-time compressive tracking. In Proceedings of the European Conference on Computer Vision, pages 864-877, Florence, Italy, Oct. 2012. doi:10.1007/978-3-642-33712-3_62. Cited on page 17.

[201] Z. Zhang. Flexible camera calibration by viewing a plane from unknown orientations. In Proceedings of the IEEE International Conference on Computer Vision, volume 1, pages 666-673, Kerkyra, Greece, Sept. 1999. doi:10.1109/ICCV.1999.791289. Cited on page 65.

[202] Z. Zhang. A flexible new technique for camera calibration. IEEE Transactions on Pattern Analysis and Machine Intelligence, 22(11):1330-1334, Nov. 2000. ISSN 0162-8828. doi:10.1109/34.888718. Cited on page 69. 


\section{PhD Dissertations \\ Division of Automatic Control \\ Linköping University}

M. Millnert: Identification and control of systems subject to abrupt changes. Thesis No. 82, 1982. ISBN 91-7372-542-0.

A. J. M. van Overbeek: On-line structure selection for the identification of multivariable systems. Thesis No. 86, 1982. ISBN 91-7372-586-2.

B. Bengtsson: On some control problems for queues. Thesis No. 87, 1982. ISBN 91-7372593-5.

S. Ljung: Fast algorithms for integral equations and least squares identification problems. Thesis No. 93, 1983. ISBN 91-7372-641-9.

H. Jonson: A Newton method for solving non-linear optimal control problems with general constraints. Thesis No. 104, 1983. ISBN 91-7372-718-0.

E. Trulsson: Adaptive control based on explicit criterion minimization. Thesis No. 106, 1983. ISBN 91-7372-728-8.

K. Nordström: Uncertainty, robustness and sensitivity reduction in the design of single input control systems. Thesis No. 162, 1987. ISBN 91-7870-170-8.

B. Wahlberg: On the identification and approximation of linear systems. Thesis No. 163, 1987. ISBN 91-7870-175-9.

S. Gunnarsson: Frequency domain aspects of modeling and control in adaptive systems. Thesis No. 194, 1988. ISBN 91-7870-380-8.

A. Isaksson: On system identification in one and two dimensions with signal processing applications. Thesis No. 196, 1988. ISBN 91-7870-383-2.

M. Viberg: Subspace fitting concepts in sensor array processing. Thesis No. 217, 1989. ISBN 91-7870-529-0.

K. Forsman: Constructive commutative algebra in nonlinear control theory. Thesis No. 261, 1991. ISBN 91-7870-827-3.

F. Gustafsson: Estimation of discrete parameters in linear systems. Thesis No. 271, 1992. ISBN 91-7870-876-1.

P. Nagy: Tools for knowledge-based signal processing with applications to system identification. Thesis No. 280, 1992. ISBN 91-7870-962-8.

T. Svensson: Mathematical tools and software for analysis and design of nonlinear control systems. Thesis No. 285, 1992. ISBN 91-7870-989-X.

S. Andersson: On dimension reduction in sensor array signal processing. Thesis No. 290, 1992. ISBN 91-7871-015-4.

H. Hjalmarsson: Aspects on incomplete modeling in system identification. Thesis No. 298, 1993. ISBN 91-7871-070-7.

I. Klein: Automatic synthesis of sequential control schemes. Thesis No. 305, 1993. ISBN 91-7871-090-1.

J.-E. Strömberg: A mode switching modelling philosophy. Thesis No. 353, 1994. ISBN 91-7871-430-3.

K. Wang Chen: Transformation and symbolic calculations in filtering and control. Thesis No. 361, 1994. ISBN 91-7871-467-2.

T. McKelvey: Identification of state-space models from time and frequency data. Thesis No. 380, 1995. ISBN 91-7871-531-8.

J. Sjöberg: Non-linear system identification with neural networks. Thesis No. 381, 1995. ISBN 91-7871-534-2.

R. Germundsson: Symbolic systems - theory, computation and applications. Thesis No. 389, 1995. ISBN 91-7871-578-4. 
P. Pucar: Modeling and segmentation using multiple models. Thesis No. 405, 1995. ISBN 91-7871-627-6.

H. Fortell: Algebraic approaches to normal forms and zero dynamics. Thesis No. 407, 1995. ISBN 91-7871-629-2.

A. Helmersson: Methods for robust gain scheduling. Thesis No. 406, 1995. ISBN 91-7871628-4.

P. Lindskog: Methods, algorithms and tools for system identification based on prior knowledge. Thesis No. 436, 1996. ISBN 91-7871-424-8.

J. Gunnarsson: Symbolic methods and tools for discrete event dynamic systems. Thesis No. 477, 1997. ISBN 91-7871-917-8.

M. Jirstrand: Constructive methods for inequality constraints in control. Thesis No. 527, 1998. ISBN 91-7219-187-2.

U. Forssell: Closed-loop identification: Methods, theory, and applications. Thesis No. 566, 1999. ISBN 91-7219-432-4.

A. Stenman: Model on demand: Algorithms, analysis and applications. Thesis No. 571, 1999. ISBN 91-7219-450-2.

N. Bergman: Recursive Bayesian estimation: Navigation and tracking applications. Thesis No. 579, 1999. ISBN 91-7219-473-1.

K. Edström: Switched bond graphs: Simulation and analysis. Thesis No. 586, 1999. ISBN 91-7219-493-6.

M. Larsson: Behavioral and structural model based approaches to discrete diagnosis. Thesis No. 608, 1999. ISBN 91-7219-615-5.

F. Gunnarsson: Power control in cellular radio systems: Analysis, design and estimation. Thesis No. 623, 2000. ISBN 91-7219-689-0.

V. Einarsson: Model checking methods for mode switching systems. Thesis No. 652, 2000. ISBN 91-7219-836-2.

M. Norrlöf: Iterative learning control: Analysis, design, and experiments. Thesis No. 653, 2000. ISBN 91-7219-837-0.

F. Tjärnström: Variance expressions and model reduction in system identification. Thesis No. 730, 2002. ISBN 91-7373-253-2.

J. Löfberg: Minimax approaches to robust model predictive control. Thesis No. 812, 2003. ISBN 91-7373-622-8.

J. Roll: Local and piecewise affine approaches to system identification. Thesis No. 802, 2003. ISBN 91-7373-608-2.

J. Elbornsson: Analysis, estimation and compensation of mismatch effects in A/D converters. Thesis No. 811, 2003. ISBN 91-7373-621-X.

O. Härkegård: Backstepping and control allocation with applications to flight control. Thesis No. 820, 2003. ISBN 91-7373-647-3.

R. Wallin: Optimization algorithms for system analysis and identification. Thesis No. 919, 2004. ISBN 91-85297-19-4.

D. Lindgren: Projection methods for classification and identification. Thesis No. 915, 2005. ISBN 91-85297-06-2.

R. Karlsson: Particle Filtering for Positioning and Tracking Applications. Thesis No. 924, 2005. ISBN 91-85297-34-8.

J. Jansson: Collision Avoidance Theory with Applications to Automotive Collision Mitigation. Thesis No. 950, 2005. ISBN 91-85299-45-6.

E. Geijer Lundin: Uplink Load in CDMA Cellular Radio Systems. Thesis No. 977, 2005. ISBN 91-85457-49-3.

M. Enqvist: Linear Models of Nonlinear Systems. Thesis No. 985, 2005. ISBN 91-8545764-7. 
T. B. Schön: Estimation of Nonlinear Dynamic Systems - Theory and Applications. Thesis No. 998, 2006. ISBN 91-85497-03-7.

I. Lind: Regressor and Structure Selection - Uses of ANOVA in System Identification. Thesis No. 1012, 2006. ISBN 91-85523-98-4.

J. Gillberg: Frequency Domain Identification of Continuous-Time Systems Reconstruction and Robustness. Thesis No. 1031, 2006. ISBN 91-85523-34-8.

M. Gerdin: Identification and Estimation for Models Described by Differential-Algebraic Equations. Thesis No. 1046, 2006. ISBN 91-85643-87-4.

C. Grönwall: Ground Object Recognition using Laser Radar Data - Geometric Fitting, Performance Analysis, and Applications. Thesis No. 1055, 2006. ISBN 91-85643-53-X.

A. Eidehall: Tracking and threat assessment for automotive collision avoidance. Thesis No. 1066, 2007. ISBN 91-85643-10-6.

F. Eng: Non-Uniform Sampling in Statistical Signal Processing. Thesis No. 1082, 2007. ISBN 978-91-85715-49-7.

E. Wernholt: Multivariable Frequency-Domain Identification of Industrial Robots. Thesis No. 1138, 2007. ISBN 978-91-85895-72-4.

D. Axehill: Integer Quadratic Programming for Control and Communication. Thesis No. 1158, 2008. ISBN 978-91-85523-03-0.

G. Hendeby: Performance and Implementation Aspects of Nonlinear Filtering. Thesis No. 1161, 2008. ISBN 978-91-7393-979-9.

J. Sjöberg: Optimal Control and Model Reduction of Nonlinear DAE Models. Thesis No. 1166, 2008. ISBN 978-91-7393-964-5.

D. Törnqvist: Estimation and Detection with Applications to Navigation. Thesis No. 1216, 2008. ISBN 978-91-7393-785-6.

P-J. Nordlund: Efficient Estimation and Detection Methods for Airborne Applications. Thesis No. 1231, 2008. ISBN 978-91-7393-720-7.

H. Tidefelt: Differential-algebraic equations and matrix-valued singular perturbation. Thesis No. 1292, 2009. ISBN 978-91-7393-479-4.

H. Ohlsson: Regularization for Sparseness and Smoothness - Applications in System Identification and Signal Processing. Thesis No. 1351, 2010. ISBN 978-91-7393-287-5.

S. Moberg: Modeling and Control of Flexible Manipulators. Thesis No. 1349, 2010. ISBN 978-91-7393-289-9.

J. Wallén: Estimation-based iterative learning control. Thesis No. 1358, 2011. ISBN 978-91-7393-255-4.

J. D. Hol: Sensor Fusion and Calibration of Inertial Sensors, Vision, Ultra-Wideband and GPS. Thesis No. 1368, 2011. ISBN 978-91-7393-197-7.

D. Ankelhed: On the Design of Low Order H-infinity Controllers. Thesis No. 1371, 2011. ISBN 978-91-7393-157-1.

C. Lundquist: Sensor Fusion for Automotive Applications. Thesis No. 1409, 2011. ISBN 978-91-7393-023-9.

P. Skoglar: Tracking and Planning for Surveillance Applications. Thesis No. 1432, 2012. ISBN 978-91-7519-941-2.

K. Granström: Extended target tracking using PHD filters. Thesis No. 1476, 2012. ISBN 978-91-7519-796-8.

C. Lyzell: Structural Reformulations in System Identification. Thesis No. 1475, 2012. ISBN 978-91-7519-800-2.

J. Callmer: Autonomous Localization in Unknown Environments. Thesis No. 1520, 2013. ISBN 978-91-7519-620-6.

D. Petersson: A Nonlinear Optimization Approach to H2-Optimal Modeling and Control. Thesis No. 1528, 2013. ISBN 978-91-7519-567-4. 
Z. Sjanic: Navigation and Mapping for Aerial Vehicles Based on Inertial and Imaging Sensors. Thesis No. 1533, 2013. ISBN 978-91-7519-553-7.

F. Lindsten: Particle Filters and Markov Chains for Learning of Dynamical Systems. Thesis No. 1530, 2013. ISBN 978-91-7519-559-9.

P. Axelsson: Sensor Fusion and Control Applied to Industrial Manipulators. Thesis No. 1585, 2014. ISBN 978-91-7519-368-7.

A. Carvalho Bittencourt: Modeling and Diagnosis of Friction and Wear in Industrial Robots. Thesis No. 1617, 2014. ISBN 978-91-7519-251-2.

M. Skoglund: Inertial Navigation and Mapping for Autonomous Vehicles. Thesis No. 1623, 2014. ISBN 978-91-7519-233-8.

S. Khoshfetrat Pakazad: Divide and Conquer: Distributed Optimization and Robustness Analysis. Thesis No. 1676, 2015. ISBN 978-91-7519-050-1.

T. Ardeshiri: Analytical Approximations for Bayesian Inference. Thesis No. 1710, 2015. ISBN 978-91-7685-930-8.

N. Wahlström: Modeling of Magnetic Fields and Extended Objects for Localization Applications. Thesis No. 1723, 2015. ISBN 978-91-7685-903-2.

J. Dahlin: Accelerating Monte Carlo methods for Bayesian inference in dynamical models. Thesis No. 1754, 2016. ISBN 978-91-7685-797-7.

M. Kok: Probabilistic modeling for sensor fusion with inertial measurements. Thesis No. 1814, 2016. ISBN 978-91-7685-621-5.

J. Linder: Indirect System Identification for Unknown Input Problems: With Applications to Ships. Thesis No. 1829, 2017. ISBN 978-91-7685-588-1.

M. Roth: Advanced Kalman Filtering Approaches to Bayesian State Estimation. Thesis No. 1832, 2017. ISBN 978-91-7685-578-2.

I. Nielsen: Structure-Exploiting Numerical Algorithms for Optimal Control. Thesis No. 1848, 2017. ISBN 978-91-7685-528-7.

D. Simon: Fighter Aircraft Maneuver Limiting Using MPC: Theory and Application. Thesis No. 1881, 2017. ISBN 978-91-7685-450-1. 